DEVELOPMENT OF A TIME-RESOLVED QUANTITATIVE SURFACE-TEMPERATURE MEASUREMENT TECHNIQUE AND ITS APPLICATION IN SHORT-DURATION WIND TUNNEL TESTING

\author{
DISSERTATION \\ zur Erlangung des mathematisch-naturwissenschaftlichen Doktorgrades \\ „Doctor rerum naturalium“ \\ der Georg-August-Universität Göttingen \\ im Promotionsprogramm ProPhys \\ der Georg-August University School of Science (GAUSS)
}

vorgelegt von

\title{
STEFFEN RISIUS
}

aus Emden 


\section{Betreuungsausschuss}

\section{Prof. Dr. Andreas Dillmann,}

Georg-August-Universität Göttingen, Institut für Nichtlineare Dynamik und Deutsches Zentrum für Luft- und Raumfahrt e.V., Institut für Aerodynamik und Strömungstechnik

Prof. Dr. Martin Rein,

Georg-August-Universität Göttingen, Institut für Nichtlineare Dynamik und Deutsches Zentrum für Luft- und Raumfahrt e.V., Institut für Aerodynamik und Strömungstechnik

\section{Dr. Stefan Hein,}

Deutsches Zentrum für Luft- und Raumfahrt e.V., Institut für Aerodynamik und Strömungstechnik, Abteilung Hochgeschwindigkeitskonfiguration (AS-HGK)

\section{Dr. Christian Klein,}

Deutsches Zentrum für Luft- und Raumfahrt e.V., Institut für Aerodynamik und Strömungstechnik, Abteilung Experimentelle Verfahren (AS-EXV)

\section{Mitglieder der Prüfungskommission}

\section{Referent: Prof. Dr. Andreas Dillmann,}

Georg-August-Universität Göttingen, Institut für Nichtlineare Dynamik und Deutsches Zentrum für Luft- und Raumfahrt e.V., Institut für Aerodynamik und Strömungstechnik

\section{Korreferent: Prof. Dr. Martin Rein,}

Georg-August-Universität Göttingen, Institut für Nichtlineare Dynamik und Deutsches Zentrum für Luft- und Raumfahrt e.V., Institut für Aerodynamik und Strömungstechnik

\section{Weitere Mitglieder der Prüfungskommission}

\section{Prof. Dr. Wolfgang Glatzel,}

Georg-August-Universität Göttingen, Institut für Astrophysik

\section{Prof. Dr. Stephan Herminghaus,}

Georg-August-Universität Göttingen, Institut für Nichtllineare Dynamik und Max-Planck-Institut für Dynamik und Selbstorganisation

\section{Prof. Dr. Wolfram Kollatschny,}

Georg-August-Universität Göttingen, Institut für Astrophysik

\section{Prof. Dr. Andreas Tilgner,}

Georg-August-Universität Göttingen, Institut für Geophysik 


\section{Acknowledgements}

The dissertation was an outcome of my research at the German Aerospace Center (DLR) in Göttingen. Without the support and advice of many people this work would have never been possible. First of all I would like to acknowledge my academic supervisor Prof. A. Dillmann and academic co-supervisors Prof. M. Rein, Dr. S. Hein and especially Dr. C. Klein for the continuous advice and support of this dissertation.

I am also very thankful to all (former) members of the Pressure- and Temperature-Sensitive Paint group in the Experimental Methods department of DLR, in particular Dr. W. Beck, Dr. M. Costantini, B. Dimond, S. Dufhaus, Prof. Y. Egami (Aichi Inst. of Techn.), Dr. R. Engler, Dr. U. Fey, Dr. U. Henne, Dr. M. Hilfer, Dr. C. Klein, W. Lang, J. Lemarechal, Prof. M. Munekata (Kumamoto University), Dr. W. Sachs, A. Weiss and Dr. D. Yorita. I am very glad and thankful to be part of this group of wonderful people, who have become far more than colleagues to me.

The wind tunnel measurement campaigns would not have been possible without the help of the colleagues M. Aschoff, Dr. M. Bruse, A. Grimme, S. Hucke, R. Kahle, M. Löhr and K. Plettenberg from the German-Dutch Wind Tunnels (DNW). I am also grateful to the Head of the Experimental Methods department, Dr. L. Koop, and many other colleagues from DLR Göttingen who supported me throughout the years, in particular J. Agocs, Dr. T. Ahlefeldt, U. Becker, C. Fuchs, Dr. S. Haxter, T. Kleindienst, Dr. S. Koch, Dr. D. Schmeling, Dr. J. Martinez Schramm, I. Micknaus, Dr. H. Rosemann, C. Rosenstock, Dr. A. Schröder and Dr. A. Wagner. For the completion of this thesis it was indispensable what I have learned from my former colleagues and advisers, in particular Prof. E. Bodenschatz, Dr. F. Di Lorenzo, Dr. S. Klein, S. Lambertz and especially Prof. H. Xu (Tsinghua University) at the Max-Planck-Institute for Dynamics and Self-Organization as well as Prof. K. Asai and Prof. H. Nagai from Tohoku University.

The good cooperation with external partners, in particular Prof. U. Beifuß and Dr. V. Ondrus (University of Hohenheim); P. Dür and G. Holst (PCO AG); Dr. P. Guntermann, A.-K. Hensch and J. Quest (ETW GmbH); S. von Deetzen and M. Müller (Deharde GmbH); Dr. S. Schaber, Dr. G. Schrauf and A. Styles (Airbus) is also acknowledged.

Last but not least I want to thank my family and friends, in particular C. Alberts, M. Boyraz, H. Buhr, Cocu family, Dr. A.\&M. Eberle, P. Federmann, Folkers family, Goldbach family, Hallenga family, K. Hank, J. Hinze, A. Jakobi, R. Kaufmann, D. Kreutzburg, M. Lüther, Moots family, Müller family, Przysucha family, S. Randahl, Rauch family, Reimold family, Risius family, K. Roskam, Sabat family, M. Schmidt, Dr. O. Schütte, Dr. I. Stroescu, Tielker family, Wiedermann family and Wild family but most of all my parents and my daughter for everything.

Thank you! 
You could not step twice into the same stream.

Heraclitus 



\begin{abstract}
In this cumulative thesis, a time-resolved quantitative temperature-sensitive paint (TSP) measurement technique was developed and used to measure temperatures at subsonic and up to hypersonic flow velocities in two different short duration wind tunnels. Based on this measurement technique it was possible to calculate the heat transfer into a wedge model in the High Enthalpy Shock Tunnel Göttingen (HEG). Furthermore, the measurement technique was developed further to determine the location of laminar-turbulent transition on the two-dimensional wind tunnel model PaLASTra, which offers a quasi-uniform streamwise pressure gradient, in the Cryogenic Ludwieg-Tube Göttingen (DNW-KRG) with temporal and spatial accuracy that had not been reached before. The quantitative temperature results obtained from TSP measurements allowed the systematic analysis of unit Reynolds number, Mach number and pressure gradient effects on laminar-turbulent transition at flow speeds from $M=0.35$ to 0.77 . The following is a list of the main findings:

1. The usability of TSP to measure temperatures on a wedge surface in a hypersonic, short-duration flow was demonstrated. The time-resolved temperature information was used to calculate heat load, making use of those assumptions generally applied to these types of flows. Both temperature and heat load results on the surface of a ramp model placed in a Mach 7.4 HEG flow are shown. A Medtherm coaxial thermocouple situated at one position on the model was used to calibrate the TSP (viz. to obtain the thermal parameter $\rho c k$ for the TSP). An average heat load in the interrogation region of $0.73 \pm 0.07 \mathrm{MWm}^{-2}$ was found. Furthermore, a detailed discussion of error sources and uncertainties was carried out.
\end{abstract}

2. The correction of temperature effects on the determined transition Reynolds numbers and critical $\mathrm{N}$-factors in the Cryogenic Ludwieg-Tube Göttingen. The temperature difference between the flow and model surface which is required for TSP transition measurements influences boundary-layer instability and the measured transition locations. It was corrected by a linearized fit which relates the adiabatic and non-adiabatic transition Reynolds numbers to the wall temperature ratios. This correction was made possible by the development of a time-resolved and quantitative surface temperature measurement with TSP. The temperature distributions measured by TSP were also used as input for boundary-layer calculations and linear stability analysis which led to a correction of temperature influences on critical $\mathrm{N}$-factors.

3. It was shown that modifications of the PaLASTra model proved to be useful in order to reduce the magnitude of pressure fluctuations, caused by flow separation at the trailing edge of the original model, below the minimum measurable quantity. The separation-induced pressure fluctuations and their influence on the location of laminar-turbulent transition was investigated in DNW-KRG. As a result, an additional aft part was installed at the trailing edge of the PaLASTra model and used for the further investigations.

4. It was found that the PaLASTra model exhibits a quasi-uniform streamwise pressure gradient, characterized by the incompressible shape factor, $H_{12}$, or the Hartree parameter, $\beta_{H}$. A linear 
dependency between $H_{12}$ (or $\beta_{H}$ ) and the transition Reynolds number was found for each combination of Mach and unit Reynolds number, this could be approximated by linear functions. These approximations were an important step to obtain the further results which are described below.

5. The unit Reynolds number effect on the transition Reynolds number was explained and quantified by the relation between the spectral level and the frequency leading to Tollmien-Schlichting (T-S) induced transition. To this end, a power law between unit Reynolds number, $R e_{1}$, and transition Reynolds number, $R e_{t r}$, was used, with $R e_{t r} \sim R e_{1}^{\alpha_{\text {III }}}$. The exponent $\alpha_{\text {III }}$ was found to range between 0.1 and 0.6 for accelerated flows, which agrees well with earlier measurements in hypersonic wind tunnels.

6. The first quantitative description of the Mach number effect (or 'compressibility effect') in a subsonic flow. This quantification was made possible by using the unique feature of DNW-KRG to be able to vary Mach number, unit Reynolds number and total temperature separately. Therefore, the systematic variation of $M, R_{1}$ and $H_{12}$ was combined with a detailed analysis of the free stream turbulence spectrum in the wind tunnel. Furthermore, it was shown that not the RMS turbulence level alone is of importance for T-S induced transition, but the spectral level in the relevant frequency range has to be considered as well.

7. The finding of the critical $N$-factor of $N_{\text {comp }} \approx 9.0$, for compressible linear stability calculations, and $N_{\text {inc }} \approx 9.5$ for incompressible calculations for the modified PaLASTra model in DNW-KRG. It was shown that the exclusive incorporation of compressibility into linear stability analysis leads to a larger deviation in the determined critical $N$-factors (maximal variation of all data points: $\Delta N_{\text {comp }}=4.90$ ), as compared to the critical $N$-factors calculated by incompressible linear stability analysis $\left(\Delta N_{i n c}=4.29\right)$.

8. A correction method was proposed that takes into account the varying influence of the spectral level of total pressure fluctuations $\left(p^{\star}\right)$ on the initial T-S wave amplitude. By this correction the standard deviations and maximal variations of the critical $N$-factors were reduced and led to compressible critical $N$-factors $\left(\Delta N_{\text {comp }, p^{\star}}=4.02\right)$ which are similar to or smaller than in the incompressible case $\left(\Delta N_{i n c, p^{\star}}=4.06\right)$.

9. It was found that the determined critical $N$-factors show a dependency on $H_{12}$ which is in agreement with earlier findings. It is plausible that this effect is related to the receptivity process, which can be reduced by incorporating the receptivity dependency of acoustic disturbances $(C)$ on incidence angles. A correction method was developed which reduces the dependency on $H_{12}$ and leads to even smaller maximal variations of the critical $N$-factors of $\Delta N_{i n c, p^{\star}, C}=3.81$ and $\Delta N_{\text {comp }, p^{\star}, C}=3.53$.

10. It was found that the correlated critical $N$-factors are dependent on Mach number. The dependency is larger for the compressible than for the incompressible $\mathrm{N}$-factors, with a variation of the mean of $\delta N_{\text {comp }} \approx 1.7$ and $\delta N_{\text {inc }} \approx 0.6$, respectively. The Mach number dependency was further reduced by the correction methods described above. 



\section{Contents}

Abstract vi vi vis

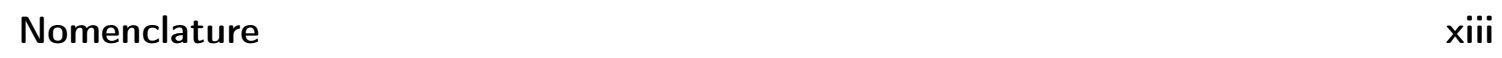

\begin{tabular}{lll}
\hline PREAMBLE & 1
\end{tabular}

1 Motivation 3

2 Introduction $\mathbf{5}$

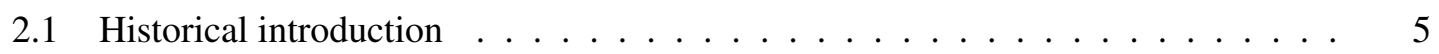

2.2 Relevance for today's world $\ldots \ldots \ldots \ldots \ldots$

2.3 Outline and major achievements of the thesis f . . . . . . . . . . . 7

2.4 Impact of the work and other achievements $\ldots \ldots \ldots \ldots$

\begin{tabular}{lll}
\hline ANALYSIS AND RESULTS & 11
\end{tabular}

3 Determination of heat transfer in a hypersonic flow (Risius et al., 2017) 13

3.1 Abstract . . . . . . . . . . . . . . . . . . . 13

3.2 Introduction . . . . . . . . . . . . . . . . . . . . . . . . . . . . . . . 14

3.3 Experimental section $\ldots \ldots \ldots \ldots \ldots \ldots$

3.4 Temperature determination using TSP $\ldots \ldots \ldots \ldots \ldots \ldots$

3.5 Determination of heat loads . . . . . . . . . . . . . . . . . . . . 19

3.5.1 Temperature sensors . . . . . . . . . . . . . . . . . . . 19

3.5 .2 Evaluation of heat transfer from Medtherms and TSP . . . . . . . . . . 19

3.6 Results and discussion . . . . . . . . . . . . . . . . . . . . . . 20

3.6 .1 HEG results; TSP images (raw data) . . . . . . . . . . . . . . . 20

3.6 .2 TSP temperature results $\ldots \ldots \ldots \ldots \ldots$

3.6 .3 TSP heat load results $\ldots \ldots \ldots \ldots \ldots$. . . . . . . . . . . . . . . . 24

3.6 .4 Accuracy of TSP temperature and heat load results . . . . . . . . . 26

3.6.4.1 Thermal parameter $\rho c k$ : material properties . . . . . . . 26

3.6 .4 .2 The interrogation area . . . . . . . . . . . . . 28

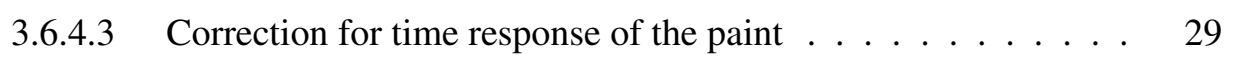

3.6.4.4 Normalized TSP and Medtherm temperatures . . . . . . . . 33

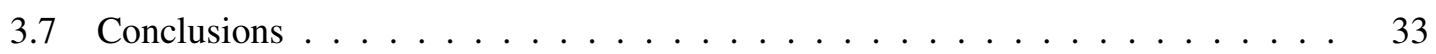


4 Mach number effects in the Cryogenic Ludwieg-Tube Göttingen (Risius et al. 2018a)

4.1 Abstract . . . . . . . . . . . . . . . . . . . . . . . . . 36

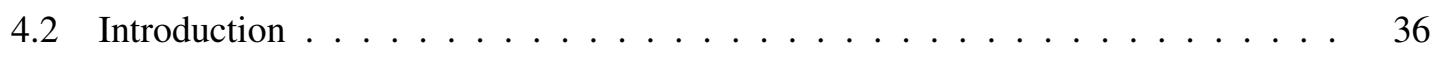

4.3 Experimental Setup and Test Conditions . . . . . . . . . . . . . . . 37

4.4 Results . . . . . . . . . . . . . . . . . . . . . . . . . . . . . . . . . . . . 41

$4.4 .1 \quad$ Mach number influence on the transition Reynolds number . . . . . . . . . 41

4.4 .2 Compressible and incompressible $N$-factor analysis . . . . . . . . . 43

4.5 Conclusions . . . . . . . . . . . . . . . . . . . . . . . . . . . . 44

5 Unit Reynolds number, Mach number and pressure gradient effects on laminarturbulent transition in two-dimensional boundary layers (Risius et al., 2018b) $\quad 47$

5.1 Abstract . . . . . . . . . . . . . . . . . . . . . 48

5.2 Introduction $\ldots \ldots \ldots \ldots \ldots \ldots \ldots$

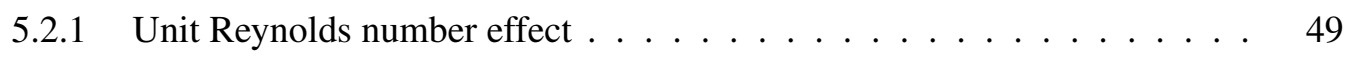

5.2 .2 Mach number effect . . . . . . . . . . . . . . . . . . . . . . . 49

5.2.3 Transition prediction by the $e^{N}$-method and correction of determined criti-

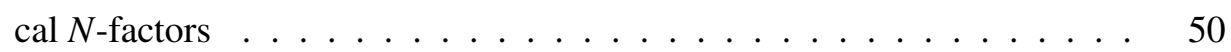

5.2 .4 Compressible and incompressible $N$-factor analysis . . . . . . . . . 51

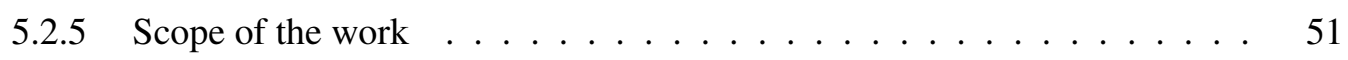

$5.2 .5 .1 \quad$ Unit Reynolds number effect $\ldots \ldots \ldots \ldots$. . . . . . 51

5.2 .5 .2 Mach number effect . . . . . . . . . . . . . 52

5.2.5.3 Correction of compressible and incompressible critical $N$-factors 52

5.2 .6 Outline of the paper . . . . . . . . . . . . . 52

5.3 Experimental setup and boundary layer computations . . . . . . . . . . 53

$5.3 .1 \quad$ The Cryogenic Ludwieg-Tube Göttingen . . . . . . . . . . . . . . . . 53

5.3 .2 The two-dimensional wind tunnel model PaLASTra . . . . . . . . . . . 53

5.3 .3 Boundary layer computations . . . . . . . . . . . . . . 55

5.3 .4 Linear stability analysis $\ldots \ldots \ldots \ldots \ldots$. . . . . . . . . . . . . . . . . . . . . . 57

5.4 Analysis of stability modifiers $\ldots \ldots \ldots \ldots \ldots \ldots$

5.4 .1 Spectral analysis of total pressure fluctuations . . . . . . . . . . 59

5.4 .2 Correction of non-adiabatic surface temperature . . . . . . . . . . 60

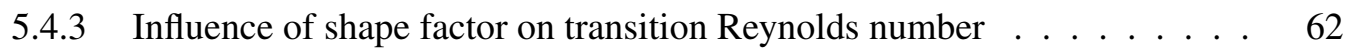

$5.4 .4 \quad$ Influence of unit Reynolds number $\left(R e_{1}^{\star}\right)$ on transition Reynolds number

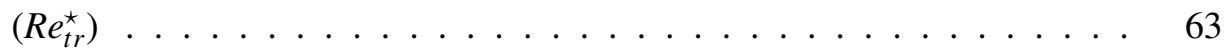

5.4.5 Relation between frequency of most amplified T-S wave at the transition location $\left(f_{t r}\right)$ and the unit Reynolds number $\left(R e_{1}^{\star}\right) \ldots \ldots \ldots$

5.4 .6 Combination of equations . . . . . . . . . . . . . . 67

5.4.6.1 Relationship between the unit Reynolds number $\left(R e_{1}^{\star}\right)$ and the spectral level of total pressure fluctuations $\left(p^{\star}\right) \ldots \ldots$. . . 68 
5.4.6.2 Transition Reynolds number $\left(R e_{t r}^{\star}\right)$ as a function of spectral level of total pressure fluctuations $\left(p^{\star}\right)$ and $H_{12} \ldots$. . . . . . . 68

5.5 Compressible and incompressible critical $N$-factors and methods for correction . 69

5.5.1 Correction of the critical $N$-factors with the spectral level of total pressure fluctuations $\left(p^{\star}\right.$-correction $)$. . . . . . . . . . . . . . . 71

5.5.2 Correction of the determined critical $N$-factors by receptivity dependency of acoustic disturbances on incidence angles $(C$-correction $)$. . . . . . . . 72

5.6 Uncertainties and repeatability . . . . . . . . . . . . . . . . . . . 74

$5.6 .1 \quad$ Uncertainties of measured parameters . . . . . . . . . . . . . 74

5.6 .2 Uncertainties in the transition Reynolds number analysis . . . . . . . . . 76

5.6 .3 Uncertainties in the critical $N$-factor analysis . . . . . . . . . . . 78

5.6 .4 Repeatability of wind tunnel entries . . . . . . . . . . . . 78

5.7 Discussion of results . . . . . . . . . . . . . . . . . . . . . 78

$5.7 .1 \quad$ Factors influencing transition Reynolds number . . . . . . . . . . . . . . 78

5.7.1.1 Unit Reynolds number effect . . . . . . . . . . . . . . 78

5.7.1.2 Mach number effect . . . . . . . . . . . . . 79

5.7 .2 Calculation and correction of the critical $N$-factor . . . . . . . . . . . . . 80

5.7.2.1 Mach number influence on compressible and incompressible critical $N$-factors . . . . . . . . . . . . . . . . 80

5.7.2.2 Influence of unit Reynolds number on critical $N$-factors . . . . 81

5.7 .3 Correction of the determined critical $N$-factors . . . . . . . . . . . 81

5.7.3.1 Correction of total pressure fluctuations . . . . . . . . . . . 82

5.7.3.2 Correction of the dependence on incidence angle of receptivity of acoustic disturbances . . . . . . . . . . . . 82

5.8 Conclusion $\ldots \ldots \ldots \ldots$. . . . . . . . . . . . . . . 83

\begin{tabular}{lr}
\hline III CONCLUSION & 87
\end{tabular}

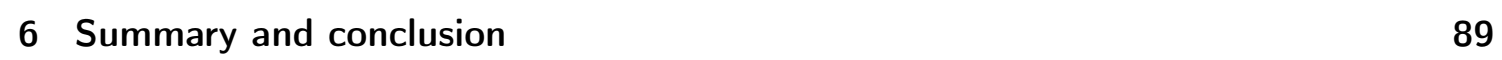

\begin{tabular}{lll}
\hline 7 & Closing remarks and outlook & 95
\end{tabular}

\begin{tabular}{lr}
\hline Bibliography & 97 \\
\hline
\end{tabular}

\begin{tabular}{|rr}
\hline IV Appendices & 109 \\
\hline
\end{tabular}

\begin{tabular}{|rr}
\hline A Further results & 111
\end{tabular} 
B Principles of temperature-sensitive paint and advances in the measurement system

B.1 Thermographic measurements and the temperature-sensitive paint (TSP) technique 117

B.1.1 Basics of photophysical processes . . . . . . . . . . . . . 117

B.1.2 Quenching . . . . . . . . . . . . . . . . . . 118

B.1.3 The Arrhenius equation $\ldots \ldots \ldots \ldots \ldots \ldots$

B.1.4 Temperature sensitive molecules . . . . . . . . . . . . . . . . . . . . 119

B.2 Setup for TSP data acquisition . . . . . . . . . . . . . . . . . . . . . . . . . . . . . . . . . . 120

B.2.1 Calibration chamber for TSP . . . . . . . . . . . . . . . 120

B.2.2 LEDs . . . . . . . . . . . . . . . . . . . . . . . 120

B.2.3 Optical filters . . . . . . . . . . . . . . . . . . . . 121

B.2.4 Cameras . . . . . . . . . . . . . . . . . . . . . . 122

B.2.5 Periscope setup in DNW-KRG . . . . . . . . . . . . . . . . . . . . 122

B.2.6 Result images of the advanced TSP measurement system . . . . . . . . . . 123

B.2.7 $\quad$ Spanwise transition detection $\ldots \ldots \ldots \ldots \ldots$. . . . . . . . . . . 124 


\section{Nomenclature}

\begin{tabular}{|c|c|}
\hline Abbreviation & Meaning \\
\hline CAD & computer aided design \\
\hline CCD & charge-coupled device \\
\hline CMOS & complementary metal-oxide-semiconductor \\
\hline $\mathrm{COCO}$ & $\begin{array}{l}\text { program to compute velocity and temperature profiles for local and non- } \\
\text { local stability analysis of compressible, conical boundary layers with } \\
\text { suction }\end{array}$ \\
\hline DLR & $\begin{array}{l}\text { Deutsches Zentrum für Luft- und Raumfahrt e.V. / German Aerospace } \\
\text { Center }\end{array}$ \\
\hline DNW-KRG & Cryogenic Ludwieg-Tube Göttingen of the German-Dutch Wind Tunnels \\
\hline ETW & European Transonic Windtunnel \\
\hline HEG & High Enthalpy Shock Tunnel Göttingen \\
\hline HIEST & High Enthalpy Shock Tunnel at Kakuda Space Center \\
\hline HST & Hypersonic Shock Tunnel \\
\hline LASER & light amplification by stimulated emission of radiation \\
\hline LED & light emitting diode \\
\hline LILO & $\begin{array}{l}\text { program to conduct linear local stability analysis based on boundary-layer } \\
\text { calculations with } C O C O\end{array}$ \\
\hline Medtherm & manufacturer of heat flux sensors (Medtherm Corporation) \\
\hline NAL & National Aerospace Laboratory of Japan \\
\hline NASA & National Aeronautics and Space Administration of the United States \\
\hline NLF & natural laminar flow \\
\hline nToPas & new three-dimensional optical pressure analysis system \\
\hline PaLASTra & $\begin{array}{l}\text { flat-plate model for the analysis of the effect on laminar-turbulent transi- } \\
\text { tion of surface imperfections, wall temperature ratio and pressure gradient }\end{array}$ \\
\hline $\mathrm{PCO}$ & camera manufacturing company: Pioneer in Cameras and Optoelectronics \\
\hline PSP & pressure-sensitive paint \\
\hline RMS & root-mean square \\
\hline SCRAMJET & supersonic combustion ramjet \\
\hline TSP & temperature-sensitive paint \\
\hline $\mathrm{T}-\mathrm{S}$ & Tollmien-Schlichting \\
\hline WT & wind tunnel \\
\hline $2 \mathrm{D}$ & two-dimensional \\
\hline $3 \mathrm{D}$ & three-dimensional \\
\hline
\end{tabular}




\section{Latin Letters}

\begin{tabular}{|c|c|}
\hline Notation & Meaning \\
\hline$A$ & area (under a curve) \\
\hline$A_{0}$ & initial amplitude of T-S wave \\
\hline$a$ & slope of a linear approximation \\
\hline$b$ & intercept of a linear approximation \\
\hline$C$ & receptivity coefficient \\
\hline$c$ & heat capacity/chord length/slope \\
\hline$F$ & dimensionless frequency: $2 \pi \cdot f \cdot v / u_{e}^{2}$ \\
\hline$f$ & frequency \\
\hline$f_{t r}$ & most amplified frequency at transition location \\
\hline$f_{\min }$ & minimal frequency \\
\hline$f_{\max }$ & maximal frequency \\
\hline$H_{12}$ & incompressible shape factor \\
\hline$h_{0}$ & specific enthalpy \\
\hline$h_{i}$ & coefficient used to approximate the shape factor \\
\hline$I$ & intensity \\
\hline$k$ & heat conductance \\
\hline$L$ & lenght scale \\
\hline$M$ & Mach number \\
\hline$N$ & (critical) $N$-factor \\
\hline$N_{\text {comp }}$ & compressible critical $N$-factor \\
\hline$N_{i n c}$ & incompressible critical $N$-factor \\
\hline$N_{p^{\star}}$ & $\begin{array}{l}\text { critical } N \text {-factor with corrected influence of normalized spectral level } \\
\text { of total pressure fluctuations }\end{array}$ \\
\hline$N_{C}$ & critical $N$-factor with corrected influence of receptivity \\
\hline$N_{p^{\star}, C}$ & $\begin{array}{l}\text { critical } N \text {-factor with corrected influence of receptivity and normalized } \\
\text { spectral level of total pressure fluctuations }\end{array}$ \\
\hline$n$ & number of camera window \\
\hline$p$ & pressure \\
\hline$p_{0}^{\prime}$ & total pressure fluctuations \\
\hline$\overline{p_{0}}$ & average total pressure fluctuations \\
\hline$p^{\star}$ & normalized spectral level of total pressure fluctuations: $p_{0}^{\prime} / \overline{p_{0}}$ \\
\hline $\operatorname{Re}$ & Reynolds number \\
\hline$R e_{1}$ & unit Reynolds number \\
\hline$R e_{1}^{\star}$ & normalized unit Reynolds number: $R e_{1} / 10^{6} \mathrm{~m}$ \\
\hline$R e_{t r}$ & transition Reynolds number based on extent of laminar flow \\
\hline$R e_{t r}^{\star}$ & normalized transition Reynolds number: $R e_{t r} / 10^{6}$ \\
\hline$R e_{t r, \text { naw }}^{\star}$ & non-adiabatic normalized transition Reynolds number \\
\hline$T$ & temperature \\
\hline
\end{tabular}




\begin{tabular}{ll} 
Notation & Meaning \\
\hline$T_{a w}$ & adiabatic wall temperature \\
$T_{n a w}$ & non-adiabatic wall temperature \\
$T u$ & turbulence level \\
$T u_{u}$ & turbulence level based on velocity $u$ \\
$T_{0}$ & temperature before gas arrival \\
$T_{t}$ & temperature at time $t$ \\
$T_{\max }$ & maximum temperature \\
$T_{\infty}$ & free stream temperature \\
$T_{C}$ & charge temperature \\
$t$ & time step \\
$t_{E}$ & camera exposure time \\
$U$ & flow velocity \\
$U_{\infty}$ & free stream velocity \\
$u_{e}$ & streamwise velocity at the boundary-layer edge \\
$x, y, z$ & Cartesian coordinates \\
$x$ & Cartesian coordinate in flow direction \\
$y$ & Cartesian coordinate in spanwise direction \\
$z$ & Cartesian coordinate perpendicular to spanwise and flow direction \\
$r e f$ & reference value \\
$m a x$ & maximum value \\
$\infty$ & free stream value \\
&
\end{tabular}

\section{Greek Letters}

\begin{tabular}{ll} 
Notation & Meaning \\
\hline$\alpha$ & slope of linear approximation, angle-of-attack \\
$\beta$ & zero intercept of linear approximation \\
$\Delta$ & difference, maximal variation \\
$\delta_{1}$ & incompressible displacement boundary-layer thickness \\
$\delta_{2}$ & incompressible momentum boundary-layer thickness \\
$\theta$ & incidence angle of acoustic waves \\
$\lambda$ & wave length \\
$\mu$ & dynamic viscosity \\
$v$ & kinematic viscosity \\
$\rho$ & density \\
$\sqrt{\rho c k}$ & thermal parameter \\
$\sigma$ & standard deviation \\
$\tau$ & time, fluorescence decay time \\
$\varphi$ & exponent for power law approach
\end{tabular}





\title{
Part I
}

\section{PREAMBLE}

\author{
1 Motivation \\ 2 Introduction
}





\section{Motivation}

Even though the computational power of super computers has been continuously increasing over the last decades, wind tunnel measurements are still the most important basis for modern aerodynamics (Melber-Wilkending et al., 2007). The complexity of fluid dynamic problems prohibits the complete simulation of high Reynolds number flows, making it necessary to conduct extensive wind tunnel tests in order to investigate aerodynamic flow features (Davidson, 2004). One important aspect of these wind tunnel measurements is the determination of pressure and temperature distributions on the surface of wind tunnel models. Within the last thirty years pressure- and temperature-sensitive paints (PSP and TSP) have been developed for the use in wind-tunnel testing. The PSP and TSP techniques allow the non-intrusive measurement of pressure and temperature distributions by detecting luminescent light, emitted from an excited PSP or TSP layer (Engler et al., 1991; Klein, 1997; Vollan and Alati, 1991).

The range of flow speeds at which wind tunnel tests are performed varies from very low subsonic flow speeds of only a few meters per second up to hypersonic flow speeds, which are several times faster than the speed of sound and relevant for vehicles (re-)entering the earth's atmosphere. Each flow speed poses its own difficulties and challenges for a measurement of the relevant aerodynamic parameters. With respect to the measurement technique TSP, such challenges are, for example, to obtain sufficient illumination of the test model and to detect the luminescent intensity in the available time frame. In the case of a hypersonic flow facility the available time frame may be limited to a few hundred micro-seconds only, as in the High Enthalpy Shock Tunnel Göttingen (HEG) (Hannemann et al. 2008). The first part of this thesis describes the time-resolved measurement of temperature distributions via TSP and the subsequent calculation of heat loads on a ramp model in HEG.

One important aspect in order to allow the transfer of results from wind tunnel measurements to free flight conditions is the preservation of dimensionless similarity parameters, such as the Mach and Reynolds numbers (Schlichting and Gersten, 2000). One way to maintain the Reynolds number for small scaled wind tunnel models is to decrease the kinematic viscosity of the fluid. A decreased kinematic viscosity can be achieved by an increase in pressure or a decrease in temperature. In the Cryogenic Ludwieg-Tube (DNW-KRG), which is operated with nitrogen as a working fluid, the effects of temperature and pressure are combined in order to adjust both Mach and Reynolds numbers to the appropriate values. The required encapsulation and the resulting space limitations in the test section of the wind tunnel pose additional challenges to TSP measurements. These challenges have to be overcome by the design of a dedicated TSP measurement system. As the second part of this thesis a new measurement setup was developed, allowing accurate and time-resolved measurement of surface temperature distributions and the reliable detection of laminar-turbulent transition locations to be made. This information is complemented by pressure 
measurements on the wind tunnel model surface in order to conduct a linear stability analysis of Tollmien-Schlichting waves in the boundary layer.

Another important aspect, to be considered for the transfer of wind tunnel measurement results to free flight conditions is the correct consideration of free flow disturbances. This aspect is especially relevant when measuring laminar-turbulent transition locations, because the excitation of TollmienSchlichting waves in the boundary layer depends strongly on the level of external disturbances. These disturbances are coupled into the boundary layer by the 'receptivity' process. In the third part of this thesis, the described improvements regarding the surface-temperature measurement system are combined with an analysis of the free stream disturbance spectrum of DNW-KRG and a linear stability analysis of Tollmien-Schlichting waves in order to correct the influence of the disturbance spectrum on laminar-turbulent transition. This aspect can be of vital importance since wind tunnels generally exhibit significantly larger disturbance levels than in free flight conditions.

In summary, the development of a time-resolved and quantitative surface temperature measurement technique and its application in short-duration wind tunnel tests, as presented in this thesis, leads to considerable results which are important for a better understanding of wind tunnel measurements and their practical applications. To better interpret the measurement results, the following ten questions shall be investigated within the presented thesis:

1. How can the temperature on a model surface be measured non-intrusively, time-resolved and quantitatively?

2. How can heat transfer be determined from the time-resolved temperature information?

3. How can locations of laminar-turbulent transition be reliably determined from surface temperature (or TSP intensity) distributions?

4. How can the influences of surface temperature distributions on the transition Reynolds number of two-dimensional flows be quantified and used as a correction?

5. What is the effect of pressure gradient variations on the transition Reynolds number and how can it be quantified?

6. How can the unit Reynolds number effect on transition Reynolds numbers be quantified?

7. What are the effects of free stream disturbances on boundary-layer transition and how can they be quantified and corrected?

8. What is the effect of compressibility on the transition Reynolds number and how can it be quantified?

9. How can transition prediction based on linear stability theory and analysis of the free stream disturbance spectrum be improved?

10. Which influences do compressible and incompressible linear stability analysis have on the correlation of the critical $N$-factors? 


\section{Introduction}

\subsection{Historical introduction}

In 1884 Osborne Reynolds published the first systematic experiments on the subject of laminarturbulent transition and stated that his results "have both a practical and a philosophical aspect" (Reynolds, 1884). As a philosophical aspect Reynolds saw the description of two possible states of motion which a fluid flowing through a pipe might take. The first state is the laminar flow, where the fluid is arranged in sheet-like parallel layers with no disruption between the layers. The second state is turbulent flow where a large mixing between the fluid elements occurs and unsteady vortices of many sizes appear in the flow. Reynolds (1884) described his observation with the words "the internal motion of water assumes one or other of two broadly distinguishable forms - either the elements of the fluid follow one another along lines of motion which lead in the most direct manner to their destination, or they eddy about in sinuous paths the most indirect possible." Reynolds (1884) also found that the question of, which one of the two states of flow takes, depends on the size of initial disturbances and on the dimensionless parameter referred to as Reynolds number

$$
R e=\frac{U L}{v},
$$

which he described in the following way: "the general character of the motion of fluids contact with solid surfaces depends on the relation between physical constant of the fluid $(v)$ and the product of the linear dimensions of the space occupied by the fluid $(L)$ and the velocity $(U)$."

As the practical aspect Reynolds (1884) considered the law of resistance in pipes, which he found rises linearly with velocity in case of a laminar flow and in a quadratic manner in the case of a turbulent flow. Until this day, both the practical and philosophical aspects remain active fields of scientific research in the case of pipes (Hof et al., 2006) but also for more complex geometries such as airfoils (Styles and Risius, 2016).

In 1904 Prandtl developed the boundary-layer theory which still forms an important basis for modern aerodynamics (Prandt1, 1905). Prandtl's boundary-layer theory divides the flow into two different regions: a region close to an object which forms the boundary layer and which is dominated by viscosity and a region further away from the object where viscosity can be neglected. This separation allows the individual approximation of the equations of fluid motion in both regions which makes their independent solution possible.

For large Reynolds numbers a temperature boundary layer can be defined together with the velocity boundary layer. Inside the thermal boundary-layer heat conductance plays a dominating role comparable to viscosity in the velocity boundary-layer. These two boundary layers need not, of course, have the same thickness. The dimensionless similarity parameters relevant for thermal 
boundary-layers are the Prandtl number and Eckart number (Schlichting and Gersten, 2000). The flow inside the boundary layer can be either laminar or turbulent which is comparable to the description of pipe flow given by Reynolds (1884). Because the viscous drag of an object moving in a fluid depends strongly on the boundary layer, it is important to determine whether the boundary layer is in a laminar or turbulent state, in order to calculate the drag and lift of the object. Therefore, it is of great practical importance to know the location where laminar-turbulent transition takes place.

A standard test case which is often used for the study of laminar-turbulent transition in boundary layers is the flow over a flat plate. A solution for a laminar boundary-layer on a flat plate was first given by Blasius (1908). It was extended to boundary layers with a pressure gradient developing in a flow over a wedge (Falkner and Skan, 1931), which leads to a pressure gradient in flow direction.

In order to investigate the process of laminar-turbulent transition in a two-dimensional boundary layer theoretically, a stability analysis of small distortions has to be conducted for the momentum equations. The basis for this stability analysis is the Orr-Sommerfeld equation, which is a differential equation of fourth order (Orr, 1907, Sommerfeld, 1908).

The stability on a flat plate was first investigated by Tollmien (1928) and Schlichting (1933) who predicted the appearance of two-dimensional waves, known as Tollmien-Schlichting (T-S) waves, leading to transition as they become unstable. The appearance of T-S waves in a Blasius boundary layer developing on a flat plate was first discovered experimentally by Schubauer and Skramstad (1948).

\subsection{Relevance for today's world}

Due to an increased anthropogenic emission of greenhouse gases the average temperature of the earth's atmosphere and oceans have already increased by about $0.8^{\circ} \mathrm{C}$ since the 1950 s (Pachauri and Mayer. 2015). Anticipated effects of the increasing global warming include rising sea levels, changing precipitation and expansion of deserts in the subtropics (Sol, 2007). The wish to reduce greenhouse gas emissions and to slow down the rise in global temperature due to aircraft trafic has led the European Commission to define the goal of a reduced $\mathrm{CO}_{2}$ emission by $75 \%$ and a reduced $\mathrm{NO}_{\mathrm{x}}$ emission by $90 \%$ per passenger kilometre until 2050, compared to a new aircraft manufactured in 2000 (ACARE, 2017). In order to reach this goal the European Commission has launched the Clean Sky Program which contains the goal to develop a Natural Laminar Flow (NLF) wing for transport aircraft (CleanSky).

Because the skin-friction coefficient of laminar flow is about one order of magnitude smaller than that in turbulent flow (Schlichting and Gersten, 2000), the NLF technology is a promising way to reduce drag of commercial aircraft by about $15 \%$ (Schrauf, 2005). Reduced aircraft drag leads to lower fuel consumption and reduced emission of $\mathrm{CO}_{2}$. The study of laminar-turbulent transition is therefore of great importance for the earth's future.

However, the universal problem of transition has many other important technical applications in aerodynamics. The extent of turbulent flow over a certain body can also influence various other parameters such as propulsive efficiency (Heister, 2016) and thermal load (Wagner et al., 2013). 
The determination of thermal load is especially relevant for spacecraft travelling at hypersonic velocities because heat loads which arise during atmospheric entry are so large that they may lead to destruction of a spacecraft during the re-entry process, such as happened with NASA's Columbia Space Shuttle in 2003. Therefore, the measurement of heat loads is of major interest for the designers of re-entry vehicles.

\subsection{Outline and major achievements of the thesis}

One major achievement of this thesis is the development of a measurement system which allows the quantitative and time-resolved measurement of temperature distributions on surfaces non-intrusively via temperature-sensitive paint. This measurement technique is combined with a calibration of the thermal properties of the TSP layer in order to determine the heat transfer into a wedge model placed in a hypersonic flow of the High Enthalpy Shock Tunnel Göttingen. Furthermore, the relevant measurement uncertainties for the described test are investigated. The development of the measurement technique and the obtained results are presented in Chapter 3 , which was published as Risius et al. (2017).

The developed measurement technique was also used in the transonic Cryogenic Ludwieg-Tube Göttingen. In this case it was not used to measure heat transfer but in order to determine the location of laminar-turbulent transition with a higher temporal and spatial resolution than had been possible in earlier measurements. Due to the operation principle of DNW-KRG a temperature step is induced by the expansion of the driving gas, which expands through the test section. This temperature step leads to a temperature difference between the flow and the model, which influences the location of laminar-turbulent transition. However, when this quantitative temperature measurement is used, the influence of the temperature distribution on laminar-turbulent transition can be determined and applied as a correction. A correction procedure was developed and applied as described in Chapters 4 and 5 and the corresponding publications Risius et al. (2018a) and Risius et al. (2018b), respectively.

The existing measurement setup was optimized to obtain results at higher signal-to-noise ratio and increase temporal and spatial resolution. As a first step, the LEDs and optical filters which are required for TSP measurements were replaced, leading to an increase in intensity by a factor of ten. The required characterization of the optical properties of TSP, optical filters and LEDs are summarized in Risius et al. (2015a) and Appendix B.

Furthermore, a new setup for TSP data acquisition in DNW-KRG was developed in order to increase the signal-to-noise ratio, as well as the spatial and temporal resolutions of TSP result images. In the new setup, the cameras are mounted behind two mirrors, which are arranged similar to the arrangement in a submarine periscope. The new setup allows a very flexible installation of larger cameras in DNW-KRG, which makes it possible to install high end camera models. The basic principle of the setup and the required pre-investigations are described in more detail in Risius (2014) and Appendix B.

For the detection of laminar-turbulent transition an algorithm was developed which uses the maximal intensity gradients in order to determine the transition locations (Costantini et al., 2018). 
This method was extended to automatically detect the transition over the complete span. The new algorithm is more reliable and leads to a better reproducibility of the results compared to a transition detection which was conducted at selected spanwise locations only. A further advantage of the new method is the quantification of spanwise variations of the detected transition locations and the corresponding root mean square of the variation (Risius et al. (2018a b) or Chapters 4 and 5 ).

Using a combination of the above measurement analysis tools, a quantitative investigation of Mach number, Reynolds number and pressure gradient effects was carried out (Chapters 4 and 5 or Risius et al. (2018a b) ). This is the first time that these effects have been quantified systematically for a transonic wind tunnel which operates at flow velocities most relevant for commercial aircraft today. Therefore, this work is relevant for research concerning the development of Natural Laminar Flow wings, with the aim of reducing fuel consumption of transport aircraft.

A standard method for transition prediction on airfoils is the $e^{N}$-method, based on linear stability theory (van Ingen, 2008). Standard tools which are used for this purpose are the boundary-layer solver COCO and the linear stability analysis tool LILO (Schrauf, 1998, 2006). These software packages were modified to incorporate the measured temperature distributions, as measured by TSP. The calculated $N$-factor distributions were correlated with transition locations in order to determine critical $N$-factors for compressible and incompressible stability theory. The new features of the linear stability analysis and its results are described in Chapters 4 and 5 or Risius et al. (2018a b).

To allow a profound investigation of the influences of free stream disturbances on laminarturbulent transition, a detailed analysis of the free flow properties is required. Measurements of the free flow in DNW-KRG have been performed by Koch (2004) and have been reanalyzed in the current study to quantify the frequency dependency of free stream total pressure fluctuations. The disturbance amplitude in the relevant frequency range of T-S waves leading to transition was used to correct the measured transition Reynolds numbers and critical $N$-factors.

\subsection{Impact of the work and other achievements}

Surface imperfections such as steps, gaps and surface waviness can influence the location of laminar-turbulent transition. For the implementation of laminar flow technology on a transport aircraft it is required to determine the maximum surface roughness requirements which allow the achievement of laminar flow on the airfoil. In order to find maximum manufacturing tolerances for steps on an airfoil, the impact of forward facing steps was investigated with the PaLASTrd 1 model in DNW-KRG and the found results were presented in Costantini et al. (2015b, 2016b).

A further requirement for the realization of laminar flow technology on transport aircraft is the achievement of laminar flow on three-dimensional models. Three-dimensional half and full aircraft models can be tested at flight Reynolds numbers in the European Transonic Windtunnel (ETW) (Green and Quest, 2011). The TSP technique was established in cryogenic environments about fifteen years ago and has become a standard method for transition detection in ETW (Fey et al., 2003; Green and Quest, 2011). Some of the work in this thesis was also used to automate

\footnotetext{
${ }^{1}$ The acronym PaLASTra stands for: flat-Plate for the analysis of the effects on LAminar-turbulent transition of Surface imperfections, wall $T$ emperature ratio and pressure gradient (Costantini et al. 2016a)
} 
and optimize the TSP measurement system at ETW (Risius et al., 2013, 2014b, 2015a) leading to more reliable and accurate wind tunnel results (Risius et al., 2014a; Wild, 2014). The obtained results were compared with calculations based on linear stability analysis and computational fluid dynamics (CFD), in order to allow a comparison of calculated and measured aircraft drag (Hue et al., 2018; Styles and Risius, 2016). Besides the investigation of transition locations, the TSP technique was also used in ETW to detect other flow features such as boundary-layer separation and footprints of vortices (Wild, 2014). Various flow features measured by TSP were also compared with CFD calculations and in-flight measurements of start and landing configurations (Risius et al., 2014a: Rudnik et al., 2015).

Results from the analysis of free stream disturbances, as mentioned above and described in Chapter 5, were also used for the analysis of free atmospheric flows at the Schneefernerhaus research station, located close to the peak of mount Zugspitze in the German Alps (Risius et al., 2015b). The obtained results were combined with a detailed analysis of turbulence parameters in free atmospheric flows and cloud microphysical properties in order to study the formation of clouds and cloud-turbulence interactions from macroscopic down to microscopic dimensions (Risius et al. 2015b; Siebert et al., 2015). 



\section{Part II}

\section{ANALYSIS AND RESULTS}

3 Determination of heat transfer in a hypersonic flow (Risius et al., 2017)

4 Mach number effects on boundary-layer transition (Risius et al., 2018a)

5 Unit Reynolds number, Mach number and pressure gradient effects on laminar-turbulent transition in two-dimensional boundary layers (Risius et al., 2018b) 



\section{Determination of heat transfer in a hypersonic flow (Risius et al., 2017)}

Citation and credit: Reprinted with permission from Experiments in Fluids, volume 58, article 117, 2017, doi:10.1007/s00348-017-2393-z, Copyright 2017, Springer International Publishing AG

Reference: Risius et al. (2017)

Title: "Determination of heat transfer into a wedge model in a hypersonic flow using temperaturesensitive paint"

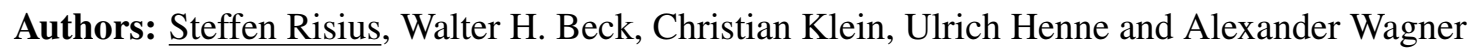

\section{Contributions of the first author:}

- processed and evaluated all measurements

- performed the calibration of temperature-sensitive paint (TSP)

- used the calibration to retrieve temperature information from the intensity images

- implemented the heat transfer calculation in the evaluation software

- applied the heat transfer calculation to the temperature results to determine heat loads

- analyzed the results by comparing heat transfer measurements obtained by TSP with results measured with a Medtherm sensor

- compared the material-based $\rho c k$ values

- made the first draft of the resulting figures and their final version

- contributed to the writing of the manuscript and edited the final version of it

- carried out the correspondence with the editor and implemented the corrections suggested by the referees

\subsection{Abstract}

Heat loads on spacecraft travelling at hypersonic speed are of major interest for their designers. Several tests using temperature-sensitive paints (TSP) have been carried out in long duration shock tunnels to determine these heat loads; generally paint layers were thin, so that certain assumptions could be invoked to enable a good estimate of the thermal parameter $\rho c k$ (a material property) 
to be obtained - the value of this parameter is needed to determine heat loads from the TSP. Very few measurements have been carried out in impulse facilities (viz. shock tunnels such as the High Enthalpy Shock Tunnel (HEG)), where test times are much shorter. Presented here are TSP temperature measurements and subsequently derived heat loads on a ramp model placed in a hypersonic flow in HEG (specific enthalpy $h_{0} \approx 3.3 \mathrm{MJ} \mathrm{kg}^{-1}$, Mach number $M=7.4$, temperature $T=277 \mathrm{~K}$, density $\rho_{\infty}=11 \mathrm{~g} / \mathrm{m}^{3}$ ). A number of fluorescence intensity images were acquired, from which, with the help of calibration data, temperature field data on the model surface were determined. From these the heat load into the surface was calculated, using an assumption of a $1 \mathrm{D}$, semi-infinite heat transfer model. $\rho c k$ for the paint was determined using an insitu calibration with a Medtherm coaxial thermocouple mounted on the model; Medtherm $\rho c k$ is known. Finally presented are sources of various measurement uncertainties, arising from: (i) estimation of $\rho c k$ (ii) intensity measurement in the chosen interrogation area (iii) paint time response.

\subsection{Introduction}

Test models coated with temperature-sensitive paints (TSP) have been used in the DLR over recent years in various wind tunnels (WT) to measure temperatures and/or temperature changes on the model surface - see Costantini et al. (2015b); Engler et al. (2000); Fey et al. (2013); Klein et al. (2005, 2013, 2014); Streit et al. (2011) for results with TSP (and also with its pressure analogue PSP - this is also referenced here since many details and difficulties of the technique are common to both TSP and PSP). Absolute temperatures as a function of time during the flow are needed to determine heat transfer from the (usually much hotter) test gas into the model surface - this will be the main topic of interest in this paper, and will be further elucidated in following sections. (Measurement of temperature changes alone can be used to determine boundary layer transition in some WT flows - this will not be further discussed here, but an example can be seen in Costantini et al. (2015b).) Since objects (missiles, space transporters and capsules, ...) moving at supersonic and especially hypersonic speeds through some atmospheric gas are exposed to very high gas temperatures behind the generated bow shock, it is essential for the optimization of the external aerodynamics and for the design of the insulation of these vehicles to be able to determine the heat loads that prevail during the various stages of the ascent and descent trajectories. The WT used are often so-called impulse facilities (such as shock tubes or tunnels), giving test times in some cases only in the millisecond (or even microsecond) range; this provides a particular challenge for the TSP technique to be able to carry out measurements in these short times. In recent years, an increased interest (Gardner et al., 2014; Gregory et al., 2014) in the study of instationary flow physical phenomena (transition, turbulence, separation, heat transfer) has led to a requirement for fast acquisition systems: high speed cameras, high power (pulsed) LED's and, above all, paints with sufficiently fast response times to enable these instationary or short-duration phenomena to be captured quantitatively and faithfully.

Some of the first measurements using TSP (and PSP) in a shock tunnel to measure heat loads (and pressure distribution) on a test model were carried out in the early 2000's by the research group of Prof. Asai (then at NAL Chofu, Japan, now at Tohoku University in Sendai, Japan): see, for 
example Nakakita et al. (2003); Ohmi et al. (2006), wherein other work in hypersonic flows is also referenced. The $0.44 \mathrm{~m}$ Hypersonic Shock Tunnel (NAL-HST) has two different operation modes to generate the shock: the Long Duration Mode (1) using fast-acting valves or the High Enthalpy Mode (2) using a bursting diaphragm, with both modes delivering flows with different durations of about $20 \mathrm{~ms}$ and $1 \mathrm{~ms}$ to $5 \mathrm{~ms}$, respectively. The TSP measurements cited in the above-mentioned papers were carried out using operation mode (2). The paint consisted of a $\mathrm{Ru}(\mathrm{phen})$ luminophore embedded in a polyacrylic acid binder. Very thin layers $(\leq 1 \mu \mathrm{m})$ were applied, and the assumption was then made that the temperature measured by TSP differs only slightly from that under the layer on the model surface - this difference was estimated to be only about $4 \%$. Hence, one could take values for the thermal parameter ( $\rho c k)$ for the model material (known) rather than that of the TSP layer (unknown) to calculate heat load from the measured TSP temperature. The thin TSP layers have the disadvantage, however, that the fluorescence intensity is quite low, although this is to some extent compensated by the long flow test times of around $20 \mathrm{~ms}$. In a recent paper by Peng et al. (2016) the results from PSP and TSP measurements carried out in a long-duration hypersonic tunnel have also been presented.

In this paper a different approach is suggested for measurements in the DLR High Enthalpy Shock Tunnel (HEG) with its much shorter test times ( $<10 \mathrm{~ms})$. The DLR Eu-based paint OV322 active (see later) used here has a high fluorescence intensity, but is much thicker $(80 \mu \mathrm{m})$ than that used in long duration tunnels, so that the assumptions for $\rho c k$ made there do not apply here. Here a Medtherm coaxial thermocouple (see later) is flush mounted with the coated test surface, so that temperature measurements from both TSP and Medtherm are available; one can use contiguous regions for temperatures from Medtherm and TSP to do an insitu calibration of $\rho c k$ for the TSP layer, using the known value for the Medtherm. Measurement uncertainties and errors are discussed in great detail in the present paper. (Whilst preparing this manuscript, a measurement using TSP in the High Enthalpy Shock Tunnel HIEST had been presented at the recent 55'th AIAA Aerospace Sciences Meeting: Nagayama et al. (2017).)

Flows in hypersonic facilities present several challenges in adapting the TSP technique to these difficult environments, especially in HEG with its test times of less than $10 \mathrm{~ms}$; however, these challenges have been met and addressed, and are discussed in a recent paper which was presented at the 2015 AIAA SciTech Meeting (Beck et al., 2015). TSP based on a different (blue-shifted) luminophore has been studied by Martinez Schramm et al. (2015) and used successfully in HEG to visualize boundary layer transition (Ozawa et al. 2015) and to examine internal SCRAMJET flows (Laurence et al., 2012).

Presented here are temperature measurements and derived heat loads using TSP on a ramp model placed in a hypersonic flow in HEG in order to demonstrate the feasibility of measuring heat transfer into the model surface under these conditions. Results from one only available HEG run are presented. A discussion of derived $\rho c k$ values for the paint is presented, along with a more detailed analysis of measurement errors arising from uncertainties in $\rho c k$ values, statistical fluctuation in the interrogation area and the finite time response of the paint relative to the camera shutter window. 


\subsection{Experimental section}

The HEG tunnel, the test model (ramp) and TSP measurement procedure have been described in greater detail elsewhere (Hannemann and Martinez Schramm, 2007; Hannemann et al., 2008), so that only a short summary will be presented here. Temperature measurements on a ramp model in a low enthalpy flow in the DLR free piston-driven shock tunnel HEG were carried out at one location with a Medtherm thermocouple and over the whole surface which was coated with the TSP paint. The used HEG test condition XIV (Hannemann et al., 2008) was chosen in order to minimize the effect of HEG background radiation (Beck et al., 2015) with specific enthalpy $h_{0} \approx 3.3 \mathrm{MJ} \mathrm{kg}^{-1}$, Mach number $M=7.4$ and the freestream properties temperature $T_{\infty}=277 \mathrm{~K}$ and density $\rho_{\infty}=11 \mathrm{~g} / \mathrm{m}^{3}$. A schematic drawing of HEG is shown in Fig. 3.1

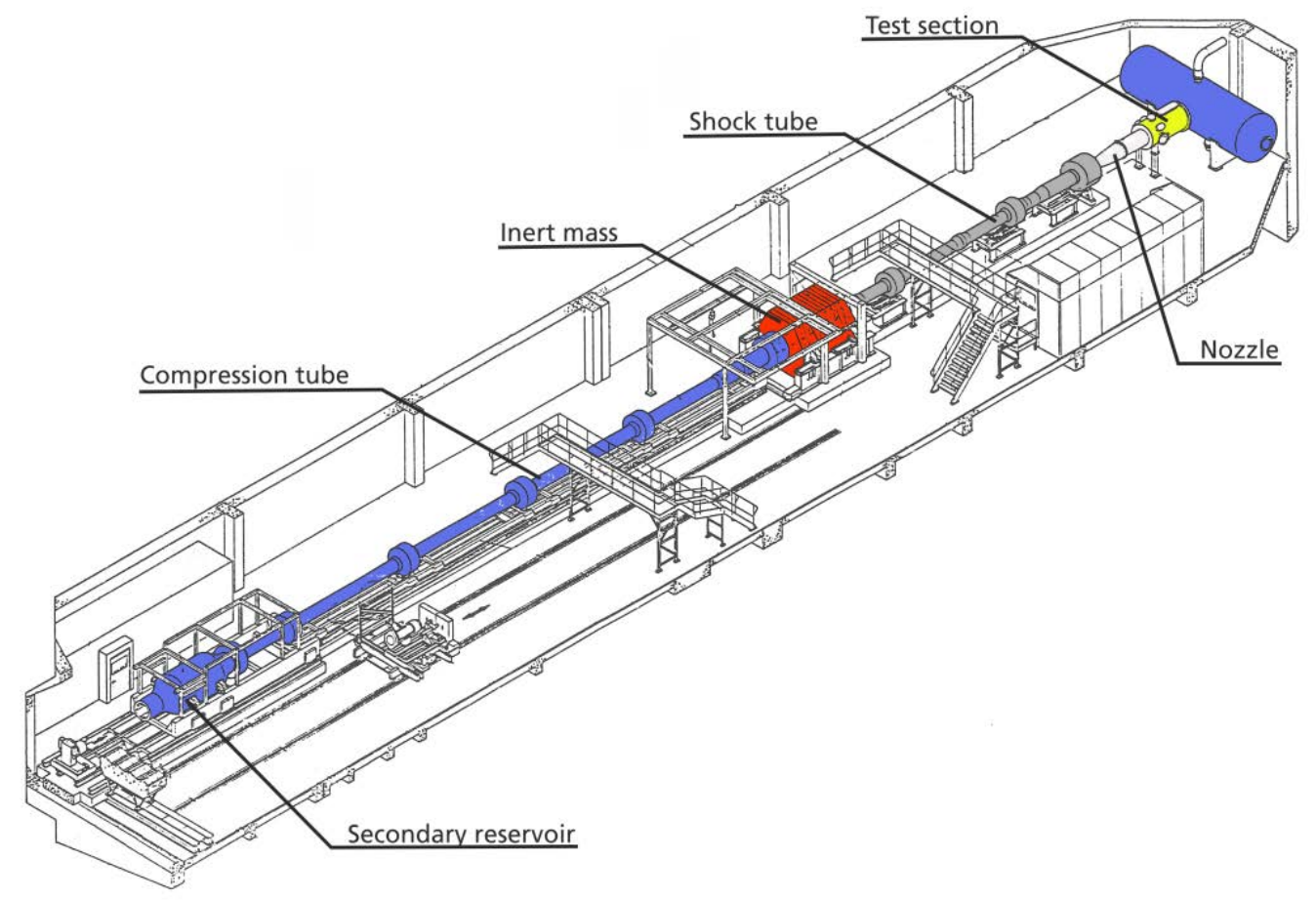

Figure 3.1: Schematic drawing of the High Enthalpy Shock Tunnel Göttingen, HEG. (Source: Wagner et al. (2013))

The ramp model, consisting of a flat aluminium plate (with dimensions $80 \mathrm{~mm} \times 20 \mathrm{~mm}$ ) with a sharp leading edge was placed at an angle of $15^{\circ}$ to the flow direction. It was coated with the DLR OV322 paint (Eu-based luminophore and polyurethane (PU) binder), which had been developed in collaboration with the University of Hohenheim (Ondrus et al., 2015). The ramp model had two small holes, one for a point-wise pressure measurement (using a Kulite ${ }^{\circledR}$ piezo-resistive pressure sensor mounted below the plate in the plate holder) and one for a point-wise temperature measurement (using the coaxial thermocouple Medtherm ${ }^{\circledR}$ protruding from the holder and inserted in the ramp model hole so as to be flush-mounted with the plate surface) - see Figs. 3.2 and 3.3 .

TSP fluorescence was excited with a Rapp ${ }^{\circledR}$ High Power LED operating at $405 \mathrm{~nm}$ in CW mode 


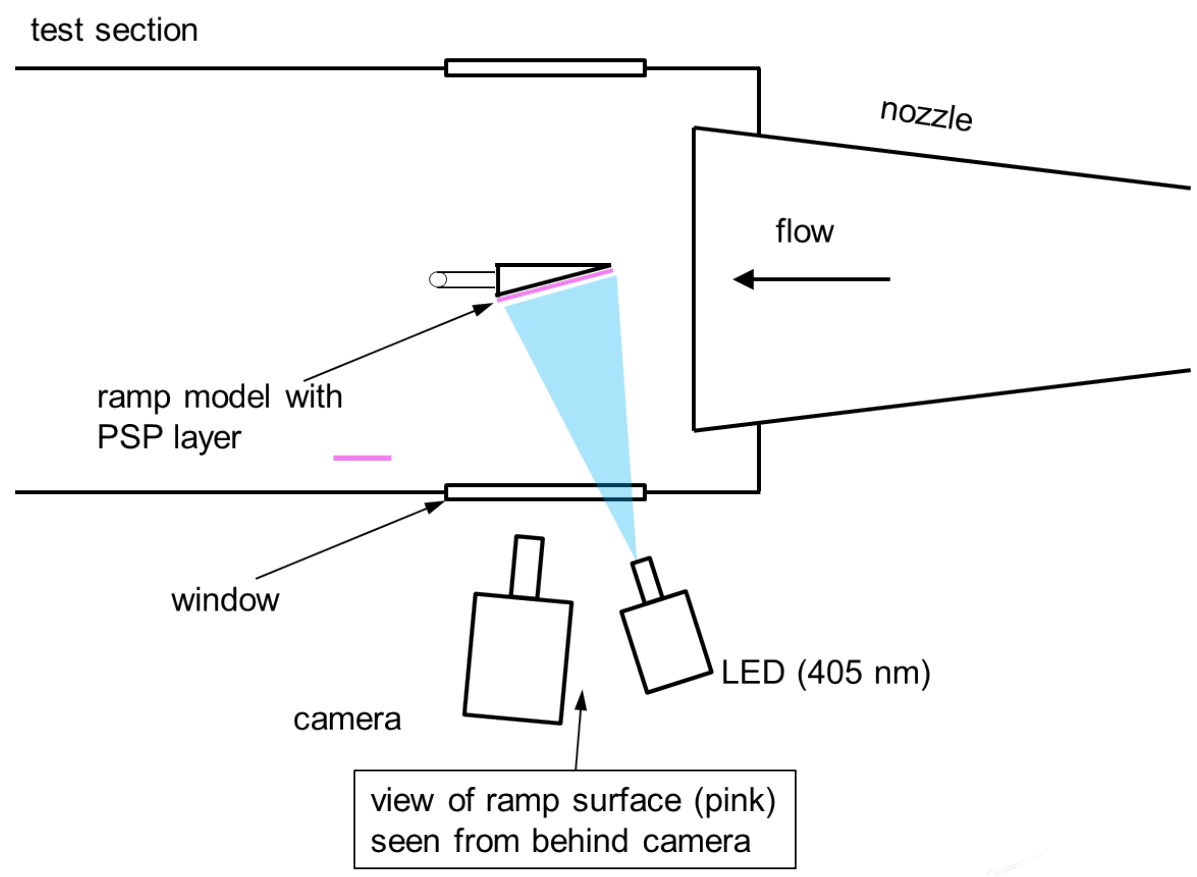

Figure 3.2: Block diagram (plan view) of experimental setup in HEG test section.

and the images were captured with a Photron ${ }^{\circledR}$ HiSpeed Camera SA1 (provided by LaVision ${ }^{\circledR}$ ) running at a sampling rate of $5 \mathrm{kHz}$ and with exposure times of $200 \mu \mathrm{s}$; recordings were carried out over $10 \mathrm{~ms}$, so that 50 images in all were obtained.

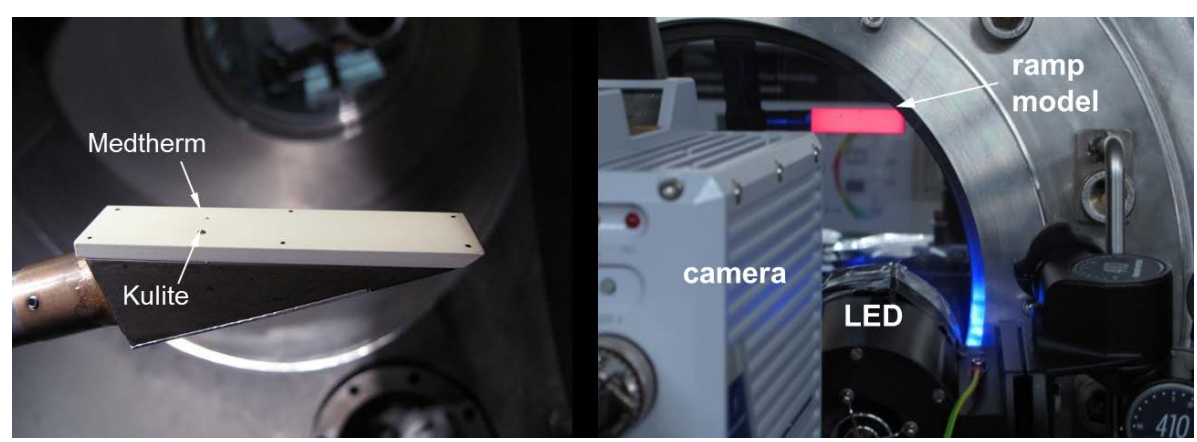

Figure 3.3: Left: Detailed photograph of the ramp model, mounted in the test section. (Flow is from right to left.) Right: Photo of irradiated ramp model (pink color) in test section, showing camera and LED light source.

\subsection{Temperature determination using TSP}

The quenching processes which reduce the intensity of the excited paint luminophore fluorescence $I(T)$ are temperature dependent: $I(T)=f(1 / T)$. Typically, using an Arrhenius formulation, one 
obtains (Liu and Sullivan, 2005):

$$
\ln \frac{I(T)}{I\left(T_{r e f}\right)}=\frac{E_{a}}{R}\left[\frac{1}{T}-\frac{1}{T_{r e f}}\right]
$$

Here $E_{a}$ is the activation energy for the non-radiative process and $R$ is the universal gas constant. In practice, Eq. 3.1 does not always hold exactly, so that one tends (Liu and Sullivan, 2005) to carry out a calibration at known $T$ (and $P$ ) and perform a fit (e.g. polynomial) of $I(T) / I\left(T_{\text {ref }}\right)$ to $T / T_{\text {ref }}$ :

$$
\frac{I\left(T_{r e f}\right)}{I(T)}=f\left(T / T_{r e f}\right)
$$

This is the standard approach adopted here, and was carried out in the laboratory using small coupons coated with the same paint as used on the test model and then calibrated in a test rig with known test conditions (viz. temperature $T$ and pressure $p$ ). A calibration plot for the OV322 paint used here is shown in Fig. 3.4 from which can be seen that this paint has good temperature sensitivity in the range $290 \mathrm{~K}$ to $340 \mathrm{~K}$ (ca. $-3 \% \mathrm{~K}^{-1}$ to $-5 \% \mathrm{~K}^{-1}$ (Ondrus et al., 2015). 1

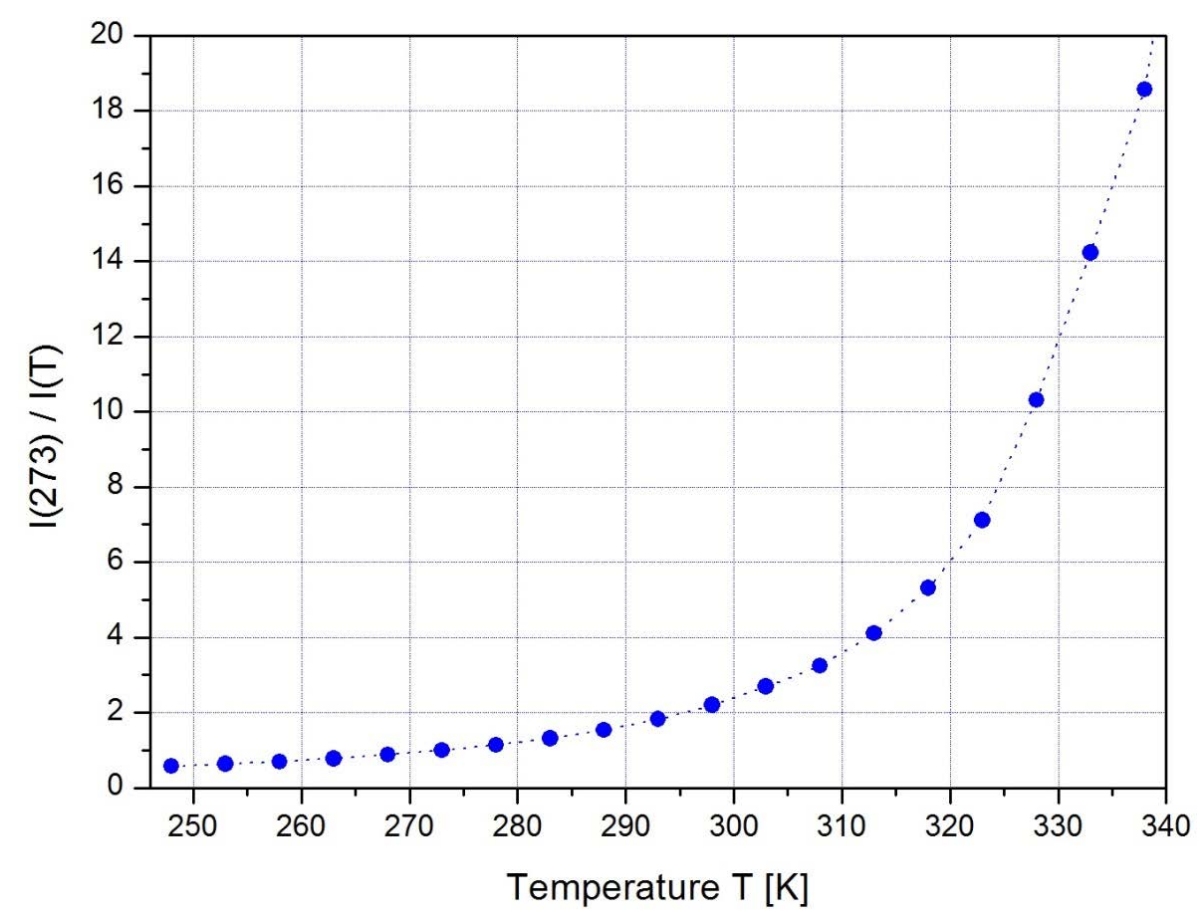

Figure 3.4: Calibration of OV322 paint: intensity ratio $I(273 K) / I(T)$ vs. temperature $T$. (Source: Ondrus et al. (2015).

With this calibration plot, and assuming that the conditions used in the HEG test were sufficiently similar (camera settings, lens settings, LED light source) to those of the calibration, one needs only one reference image in HEG to obtain the temperature results for all other images; these reference images in HEG are those which are obtained before gas arrival (at $t<1 \mathrm{~ms}$ ) - see Fig. 3.5 and later discussion in Sects. 3.6.1 and 3.6.2.

\footnotetext{
${ }^{1}$ The normalization with a different temperature would also be possible, which would lead to a constant multiplicative factor. See for example Ondrus et al. (2015).
} 


\subsection{Determination of heat loads}

\subsubsection{Temperature sensors}

Measurement of heat transfer into a model surface for hypersonic flows of high enthalpy (up to $22 \mathrm{MJ} \mathrm{kg}^{-1}$ ) and short duration (down to $100 \mu \mathrm{s}$ ) places stringent requirements on the performance of the adopted temperature sensors: they must have a fast response and be able to survive the extreme environments of these flows (especially the high temperatures and, in some cases, presence of foreign particulate matter). Over the years coaxial thermoelements have become established as the sensor of choice in HEG (and most shock tunnels) to fulfil the abovementioned requirements. The firm Medtherm supplies these sensors, and also provides a calibration (see later) to be used for the evaluation of heat transfer from temperature measurements. Obviously, these sensors yield point-wise measurements only, so that a test model must be equipped with many to obtain overall heat loads; this may be difficult, especially with some complex geometries. The advantage of a field measurement technique such as TSP lies clearly to hand.

\subsubsection{Evaluation of heat transfer from Medtherms and TSP}

Following an approach suggested by Schultz and Jones (1973), with a modification by Cook and Felderman (1966), two key assumptions were made to obtain a simple relationship for determining heat transfer from temperature measurements:

1. Heat transfer into the model surface (or paint) is one-dimensional;

2. The surface is assumed to be semi-infinite in depth.

Obviously these requirements are only met if the time over which measurement occurs is very short - for the typical test times in shock tunnels $(<10 \mathrm{~ms})$, it can be shown (Martinez Schramm et al. 2015) that these assumptions are valid. Following Hannemann and Martinez Schramm (2007) (wherein a good discussion of heat transfer measurements in shock tunnels can be found), the following equation for heat transfer has been derived:

$$
q_{w}(t)=\sqrt{\frac{\rho c k}{\pi}}\left[\frac{T(t)}{\sqrt{t}}+\frac{1}{2} \int_{0}^{t} \frac{T(t)-T(\tau)}{(t-\tau)^{3 / 2}} d \tau\right]
$$

where $T=$ measured temperature, $\rho=$ material density; $c=$ heat capacity; $k=$ heat conductivity; $\tau=$ time; $t=$ integration time step. The factor $\rho c k$ is also known as the thermal parameter, and is strictly a function of the (sensor) material properties only. This is not fully correct, since $c$ and $k$ may also have temperature dependence - see discussion in Sect. 3.6.4. Since there are no available calibration values for $\rho c k$ of the TSP paint (but see later), the approach adopted here is the following: using temperatures measured by the Medtherm sensor, and using its known $\rho c k$ value (as supplied by the manufacturer: $\rho c k=7.95 \times 10^{7} \mathrm{~kg}^{2} \mathrm{~K}^{-2} \mathrm{~s}^{-5}$ ), determine the heat load at its position using a discretized form of Eq. 3.3. Then assume that the heat load in an adjoining interrogation region (see Fig. 3.5, to be discussed later) is the same as for the Medtherm location, so that, by adjusting (varying) the $\rho c k$ value for the TSP paint in this region, the same heat load as 
for the Medtherm is determined. This is effectively an insitu calibration of the TSP paint, with the found $\rho c k$ being assumed to be the same and applicable over all the whole coated surface.

\subsection{Results and discussion}

This section addresses the following: raw TSP results; TSP temperature results; TSP heat load results; an estimation of the accuracy of and uncertainties in the TSP temperature and heat load results.

\subsubsection{HEG results; TSP images (raw data)}

The results from only one HEG run were available, so that measurements of reproducibility could not be carried out. The temperature (Medtherm) and adjacent pressure (Kulite) measurements at the respective sensor locations (as shown in the inset in Fig. 3.5 are given in Fig. 3.7, temperature is plotted as $\Delta T$ (where $T_{0}=293 \mathrm{~K}$ before gas arrival) as a function of time. The bump seen peaking at $2 \mathrm{~ms}$ in the Medtherm trace (also in the Kulite trace, but less marked) is due to the nozzle starting processes for this HEG condition. Fifty TSP images were recorded, one every $200 \mu$ s, over the $10 \mathrm{~ms}$ test time period. Fig. 3.5 shows one of these raw images (with only background correction applied, but with no correction by a reference image).

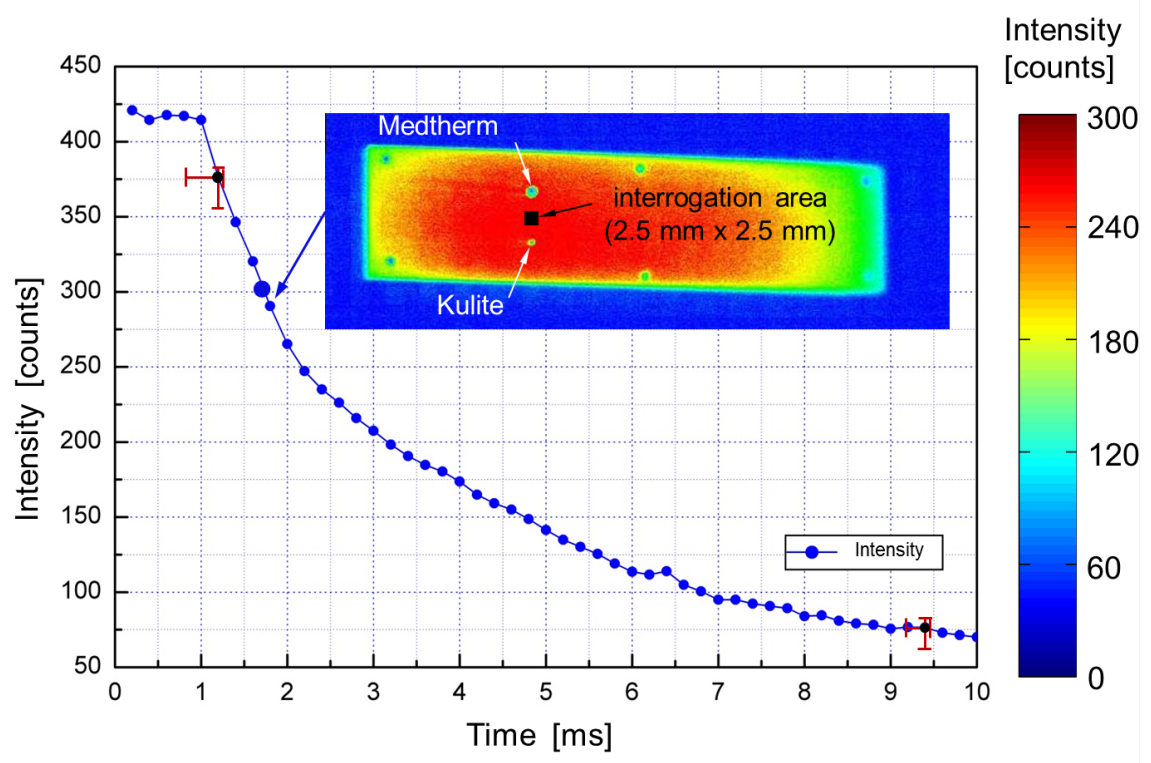

Figure 3.5: Average intensity (counts) in interrogation area vs. time (ms) for all 50 recorded raw images. Intensity and time error bars - note asymmetry - for early and late times are shown. A detailed discussion about the measurement uncertainty is given in Sect. 3.6.4.3. Inset: raw unprocessed image at $t=1.8 \mathrm{~ms}$ showing interrogation area. (Flow in the image is from right to left) 
As mentioned before, and for reasons alluded to there, a small (square) interrogation region close to the Medtherm sensor location was chosen; it was situated equidistant between the Medtherm and Kulite sensors, and had a size of $15 \times 15$ pixels, which corresponds to an area on the ramp of approximately $2.5 \mathrm{~mm} \times 2.5 \mathrm{~mm}$ - see inset in Fig. 3.5. The average (over all pixels) intensity in this region was determined for all 50 images, and then plotted as a function of time, as also shown in Fig. 3.5. (Intensity and time error bars - note asymmetry - for early and late times are shown: for a discussion, see Sect. 3.6.4.3.) The abscissa time zero (origin) corresponds to the arrival of the shock wave at the end of the driven section, at which time data recording is triggered. It can be seen that it takes about $1 \mathrm{~ms}$ for the test gas to arrive at the test section window; hence, for times less than $1 \mathrm{~ms}$, the intensity is at a maximum, after which it subsequently drops as the model surface temperature increases.

The results at $t<1 \mathrm{~ms}$ will later be used as reference values, since the conditions (viz. temperature) are well-known at these early times before the test gas has arrived. They will also be used to apply intensity distribution corrections to the results at $t>1 \mathrm{~ms}$, allowing for various geometrical effects (camera angle and settings), and for the uneven LED illumination - see Sect. 3.5.2. Interestingly, the bump seen clearly in the Medtherm result at $t=2 \mathrm{~ms}$, as referred to before (see Figs. 3.6 and 3.7), appears as a change of gradient in the TSP result at $t \approx 2.5 \mathrm{~ms}-$ see Fig. 3.8. This slight time shift between the Medtherm and TSP results is due to the finite response time of the TSP paint, and will be discussed in more detail in Sect. 3.6.4.3 The evolution (drop) of intensity is as expected from the model described by Eq. 3.3 and with the inherent assumptions referred to before. Note from Fig. 3.5 that the intensities after $7 \mathrm{~ms}$ are only about 100 counts or less, so that the accuracy of the temperature determination will be lower than at early times. This is not a serious restriction, however, since typically the customarily adopted HEG test time window lies much earlier than this (around $3 \mathrm{~ms}$ to $4 \mathrm{~ms}$ ) (see for example Laurence et al. (2014)); the flow at later times may be influenced by effects such as driver gas arrival and presence of other disturbances such as boundary layer leakage, arrival of the contact surface or of the reflected expansion wave, and so is often not further considered.

The change in intensity distribution on the model surface over time, brought about by the increasing surface temperature, can be clearly seen in the four raw images shown in Fig. 3.8 for the times $t=1.8 \mathrm{~ms}, 2.6 \mathrm{~ms}, 5.0 \mathrm{~ms}$ and $9.0 \mathrm{~ms}$. As expected, the average intensity drops steadily. At this stage, however, without application of the reference image corrections referred to before, nothing can be inferred about the change of temperature as a function of time over the surface this will be addressed in the next section. Nevertheless, one can already see some physical features: for example, a wake downstream of the Medtherm (its position is shown in Fig. 3.5), clearly visible at $t=2.6 \mathrm{~ms}$. The wake most likely arises from the sensor being mounted not fully flush with the paint surface, but recessed slightly (by about $10 \mu \mathrm{m}$ to $100 \mu \mathrm{m}$ ) below it ${ }^{2}$ Note again that the results at later times are most likely subject to disturbances which influence the state of the test gas; in spite of this, the development in intensity over time all the way up to $10 \mathrm{~ms}$ looks reasonable and

\footnotetext{
${ }^{2}$ The slight dent at the sensor location is not expected to influence the temperature and heat flux measurements of the Medtherm sensor significantly, since the boundary layer thickness at the sensor location is expected to be at least one order of magnitude larger.
} 


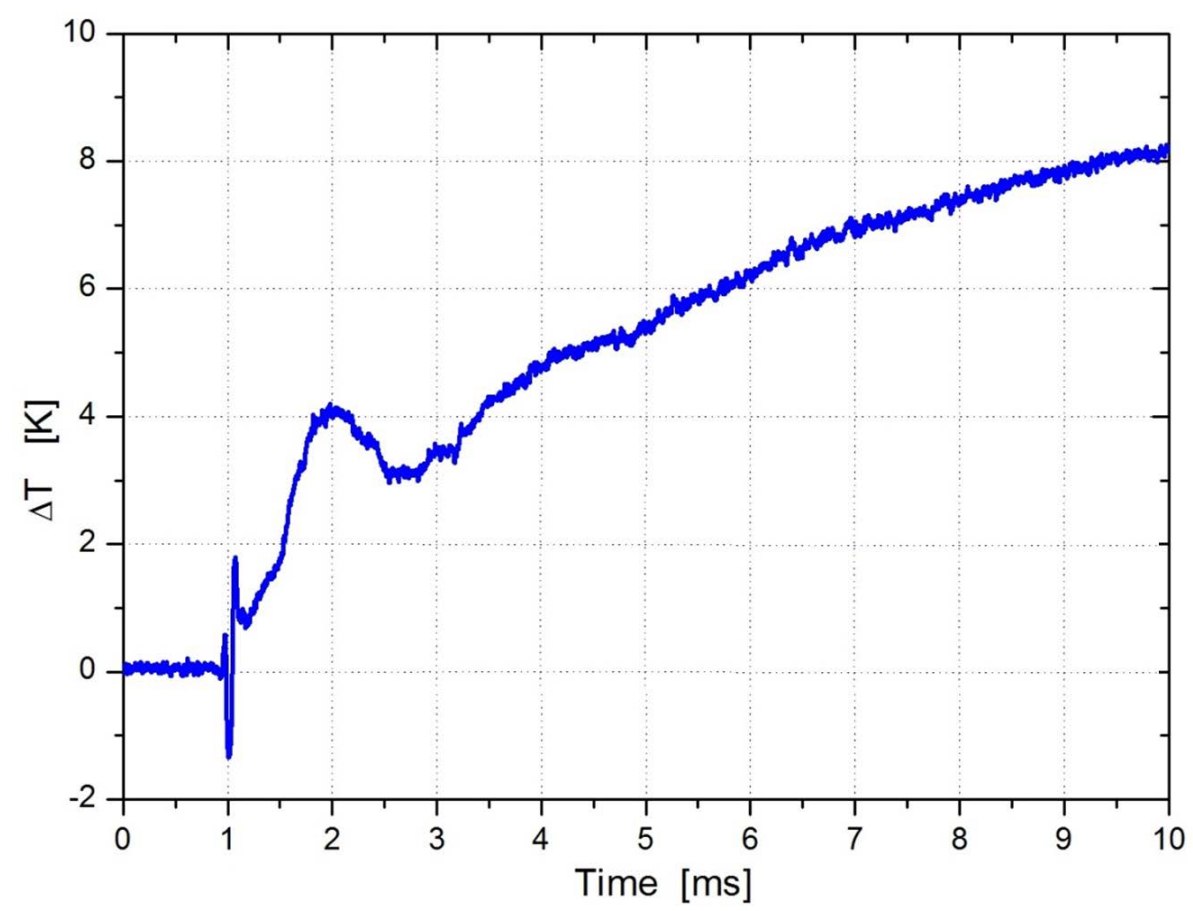

Figure 3.6: Temperature change $\Delta T$ measured by a Medtherm sensor on ramp model in this HEG run.

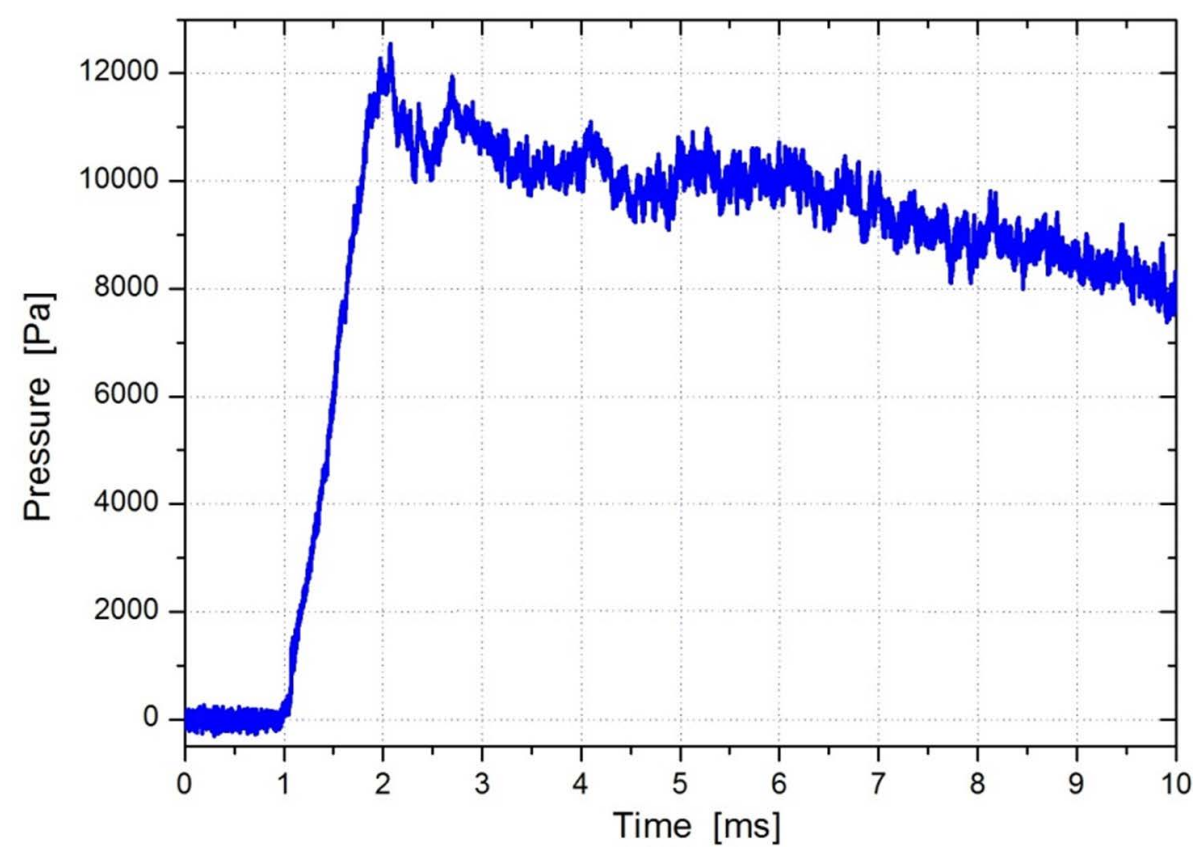

Figure 3.7: Pressure change $P$ measured by a Kulite sensor on ramp model in this HEG run.

believable. 


\subsubsection{TSP temperature results}

Using the first four recorded images as reference $\left(T_{r e f}=293 \mathrm{~K}\right)$, and with the help of the calibration plot shown in Fig. 3.4. one can then obtain temperatures over the whole model surface for all remaining 46 images. Ten results at different times $(0.4 \mathrm{~ms}, 2 \mathrm{~ms}, 3 \mathrm{~ms}, 4 \mathrm{~ms}, 5 \mathrm{~ms}, 6 \mathrm{~ms}, 7 \mathrm{~ms}$, $8 \mathrm{~ms}, 9 \mathrm{~ms}$ and $10 \mathrm{~ms}$ ) are shown in Fig. 3.9. where the first image (at $t=0.4 \mathrm{~ms}$ ) is a typical reference image before gas arrival. (The apparent asymmetry (e.g. see direction of the streak wakes) is due to non-parallel alignment of the wedge relative to the flow direction, which can already be inferred from the non-horizontal orientation of the raw images seen in Figs. 3.5 and 3.8.)

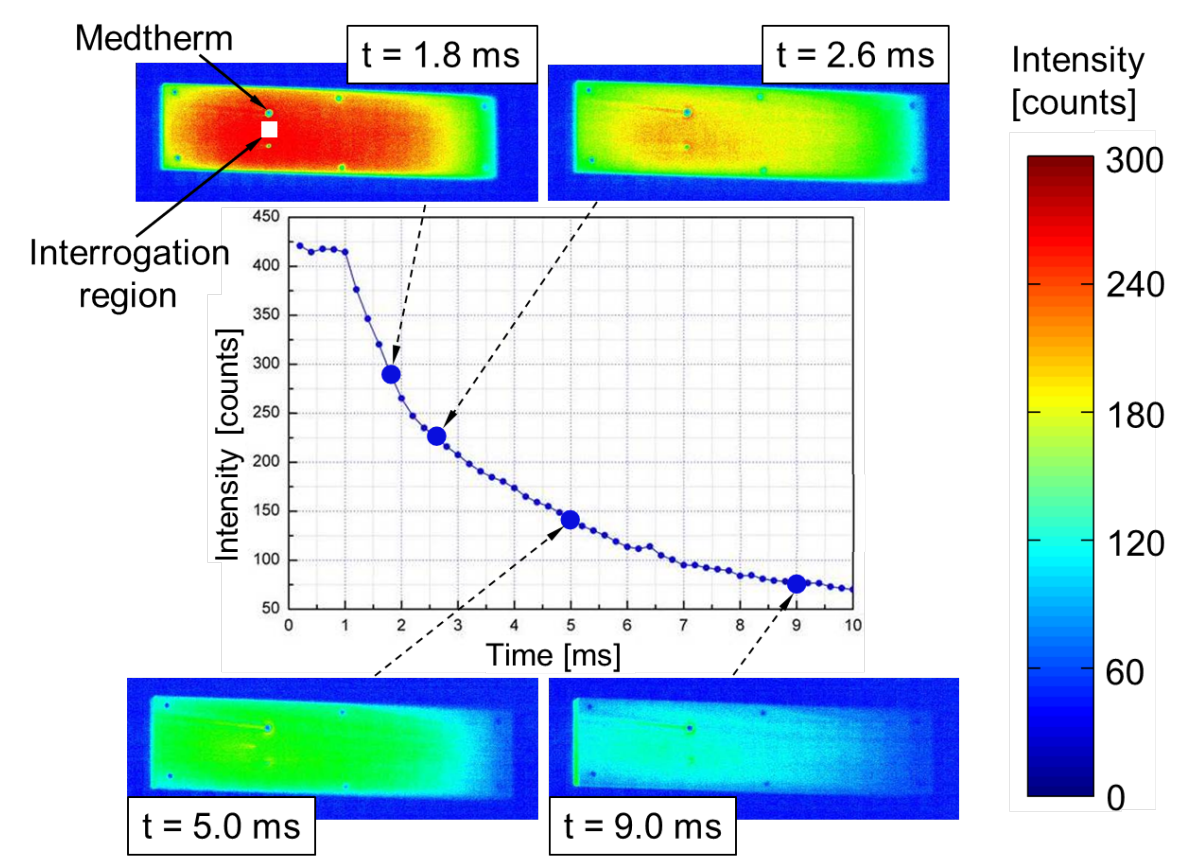

Figure 3.8: Average intensity (counts) in interrogation region vs. time (ms) for all 50 recorded raw images, showing four sample raw intensity images at times shown. (Flow in these images is from right to left)

Quite clearly one can see the development of temperature on the surface over time. (The result at $t=0.4 \mathrm{~ms}$ corresponds to a reference image, viz. before gas arrival.) Results for $t>5 \mathrm{~ms}$ suggest temperatures in excess of $330 \mathrm{~K}$ close to the leading edge; these lie outside the calibration range, so that the TSP results close to the leading edge for these late times can only be seen as semi-quantitative. However, it should be noted that the used TSP has been shown to work up to $380 \mathrm{~K}$ in a laboratory environment (Ondrus et al., 2015).

One can now plot the obtained average TSP temperatures in the interrogation region (referred to before) for all 46 images and compare the temperature development with the Medtherm results this comparison is shown in Fig. 3.10 . (Temperature and time error bars for early and late times are shown: for a discussion, see later.) Even though the TSP results after $t=2.5 \mathrm{~ms}$ look good and follow the expected trend, nevertheless recall the remarks made earlier. The difference in development of the actual temperature values obviously arises from the vastly different thermal 


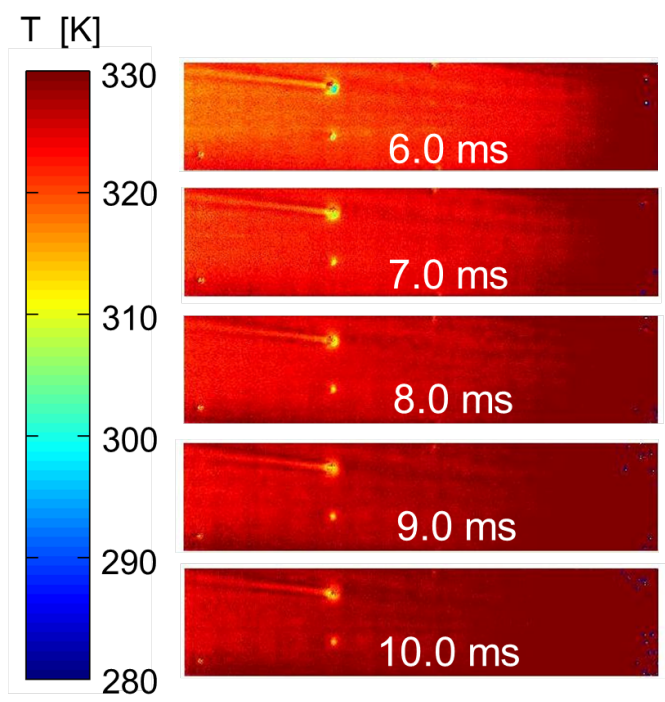

Figure 3.9: TSP temperature results ( $2 \mathrm{D}$ images) over the whole model surface for 10 different times during the HEG run. (Flow in these images is from right to left)

properties $(\rho c k)$ of the Medtherm and TSP paint. This becomes clear from Table 3.1, where $\rho, c$ and $k$ values $\left(\right.$ Eng, 2015) for three typical materials are listed, along with the products $\rho c k$ and $(\rho c k)^{1 / 2}$ - recall the presence of $(\rho c k)^{1 / 2}$ in Eq. 3.3. These materials have been chosen as 'representative': $\mathrm{Al}$ represents the test model material, Ni the Medtherm and PMMA (polymethylmethacrylate, a polymer commonly used in TSP) the paint. The values of $(\rho c k)$ and $(\rho c k)^{1 / 2}$ for the Medtherm, as supplied by the manufacturer, are also shown. (An aside: note that $\mathrm{Ni}$ has different $(\rho c k)$ values to that of the Medtherm. This is not surprising, since the Medtherm is not made of just $\mathrm{Ni}$, and some abraded dust from its test preparation has also been rubbed into the sensor.) The final row shows the values of $(\rho c k)^{1 / 2}$ for the various materials normalized to the value $(\rho c k)_{\text {PMMA }}^{1 / 2}$. As can be seen, mainly the much smaller $\mathrm{k}$ (heat conductance) value for PMMA compared with the other three materials leads also to the vastly different $(\rho c k)^{1 / 2}$ values - factors are up to about 40 times larger. The polymer based paint (represented by PMMA in Table 3.1 cannot conduct away the absorbed heat quickly enough, so that the paint surface heats up to higher values compared to those measured by the Medtherm.

\subsubsection{TSP heat load results}

Using the discretized form of Eq. 3.3 referred to before, the TSP temperature results shown in Fig. 3.9 can now be processed to give the heat loads on the model surface. This local heat load can be determined from the Medtherm measurement at its position (see Fig. 3.5 for location), so that the TSP average temperatures over time in the adjacent interrogation region can be used to determine a local heat load from TSP measurements, with $\rho c k_{\text {insitu }}$ being varied as a parameter to yield the same heat load from both the TSP and the adjacent Medtherm measurements - this is effectively an insitu calibration of the TSP paint using the Medtherm. The value obtained for the TSP paint with this approach was $\rho c k_{\text {insitu }}=4.5 \times 10^{6} \mathrm{~kg}^{2} \mathrm{~K}^{-2} \mathrm{~s}^{-5}$. It is again assumed that this 


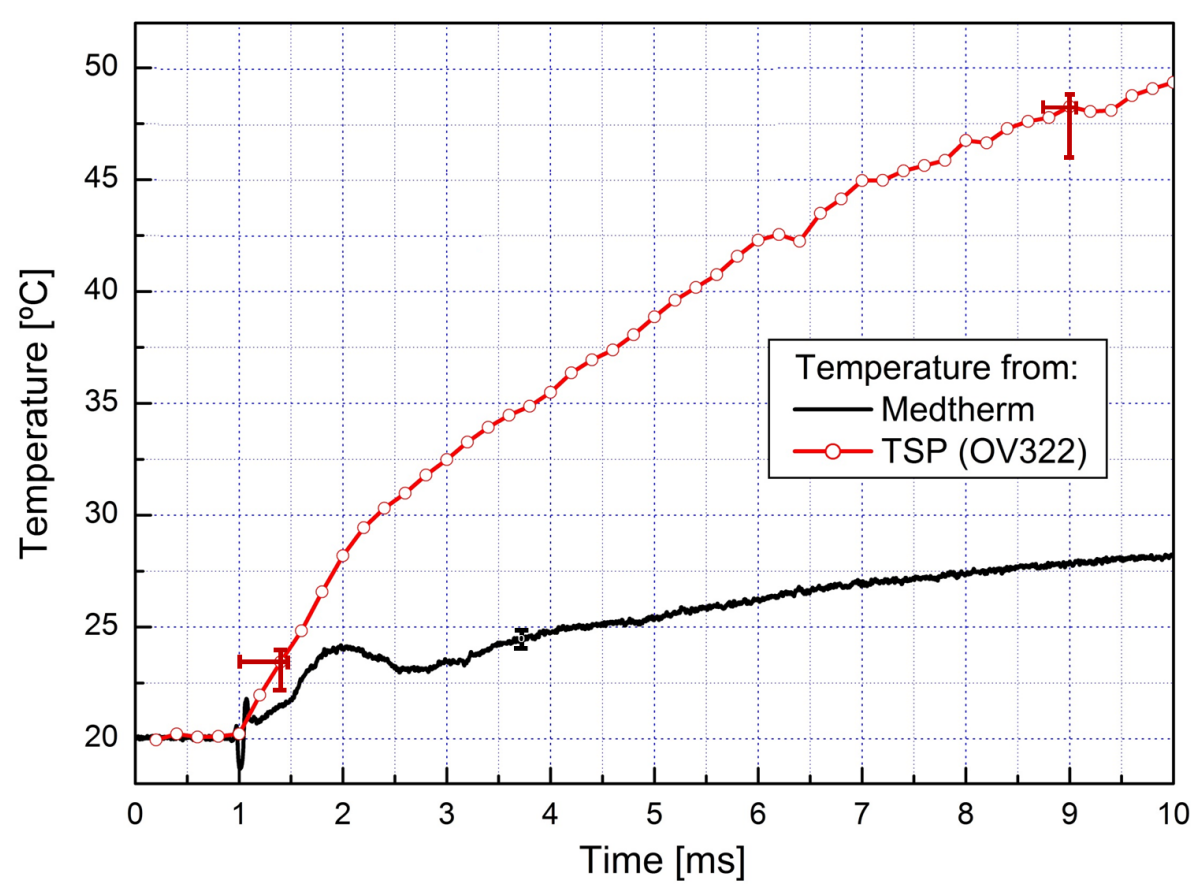

Figure 3.10: Medtherm and adjacent TSP temperatures vs. time in the HEG run, the latter using TSP calibration data. (Average TSP temperature in the interrogation area - see Fig. 3.5.)

value of $\rho c k$ pertains over the whole model surface and over the whole time, so that heat loads for all 46 images (from $t=1 \mathrm{~ms}$ to $10 \mathrm{~ms}$ ) over the whole ramp surface can be obtained. (The validity and inherent uncertainties of this assumption will be further discussed in Sect. 3.6.4.) Ten sample heat load results at different times $(0.4 \mathrm{~ms}, 2 \mathrm{~ms}, 3 \mathrm{~ms}, 4 \mathrm{~ms}, 5 \mathrm{~ms}, 6 \mathrm{~ms}, 7 \mathrm{~ms}, 8 \mathrm{~ms}, 9 \mathrm{~ms}$ and $10 \mathrm{~ms}$ ), as already shown for temperatures in Fig. 3.9. are shown in Fig. 3.11. (For the image before gas arrival at $t=0.4 \mathrm{~ms}$ the heat load is obviously zero.) Note that maximum heat loads of $1.6 \mathrm{MWm}^{-2}$ and more are obtained in the upstream (right hand side) positions on the model, even at earlier times (but recall earlier comments on paint calibration and possible damage).

As before with temperature (Fig. 3.10), one can now compare the heat load obtained from Medtherm with that from TSP in its adjacent interrogation region. This comparison is shown in Fig. 3.12. (Average error bars in time and heat transfer are shown: see discussion later.)

Here, at times after $t \approx 3 \mathrm{~ms}$ (i.e. after the initial disturbances seen in the Medtherm results), both heat load results are the same over all times and have average values of $0.73 \pm 0.07 \mathrm{MWm}^{-2}$ for TSP and $0.70 \pm 0.08 \mathrm{MWm}^{-2}$ for Medtherm. But recall: this must obviously be so, since the Medtherm results had been used to calibrate the TSP, so that the agreement has been 'forced'. Nevertheless, the constancy and agreement of heat loads over the whole time for both TSP and Medtherm is very encouraging. The bump in the Medtherm result at $t=1.6 \mathrm{~ms}$ to $2.0 \mathrm{~ms}$ correlates well with the smaller one in the TSP result at $t=2 \mathrm{~ms}$ when one considers the TSP negative time correction of $0.4 \mathrm{~ms}$ due to the finite paint response time - this will be discussed later in Sect. 3.6.4. 
Table 3.1: Comparison of material properties ( $\rho$ density, $c$ heat capacity, $k$ heat conductance) and the thermal parameter $(\rho c k)^{1 / 2}$ for nickel Ni (representing the Medtherm thermoelement), aluminum Al (test model) and PMMA (a typical TSP paint polymer). (Source: see text)

\begin{tabular}{llllll}
\hline Property & Units & Medtherm & $\mathrm{Ni}$ & $\mathrm{Al}$ & PMMA \\
\hline Density $\rho$ & $\mathrm{kg} / \mathrm{m}^{3}$ & - & 8800 & 2712 & 950 \\
Heat capacity & $\mathrm{Jkg}^{-1} \mathrm{~K}^{-1}$ & - & 540 & 870 & 1450 \\
Heat conductance & $\mathrm{Js}^{-1} \mathrm{~K}^{-1} \mathrm{~m}^{-1}$ & - & 90 & 110 & 0.18 \\
$\rho c k$ & $\mathrm{~J}^{2} \mathrm{~m}^{-4} \mathrm{~K}^{-2} \mathrm{~s}^{-1}$ & & & & \\
& $\left(\mathrm{~kg}^{2} \mathrm{~K}^{-2} \mathrm{~s}^{-5}\right)$ & $7.95 \times 10^{7}$ & $4.3 \times 10^{8}$ & $2.6 \times 10^{8}$ & $2.5 \times 10^{5}$ \\
$\sqrt{\rho c k}$ & $\mathrm{Jm}^{-2} \mathrm{~K}^{-1} \mathrm{~s}^{-1 / 2}$ & 8915 & 20700 & 16000 & 500 \\
$\sqrt{\rho c k} / \sqrt{(\rho c k)_{P M M A}}$ & $\left(\mathrm{kgK}^{-1} \mathrm{~s}^{-5 / 2}\right)$ & & 41 & 32 & 1 \\
\hline
\end{tabular}

\subsubsection{Accuracy of TSP temperature and heat load results}

Here an attempt will be made to address and identify possible sources of errors leading to uncertainties in the derived temperature and heat load results. These sources are both systematic and random. The final goal will be to determine confidence limits for these measured properties; they have already been shown in the foregoing plots as error bars. Specific other sources such as stray illumination (considered the major problem with quantitative TSP in shock tunnels) and particles (as dust) are not considered explicitly here; they have been discussed elsewhere (Beck et al., 2015). However, for the HEG run condition chosen here (low $h_{0}$, low $\rho_{\infty}$ ), the influence of these sources was minimal and could therefore be neglected here.

\subsubsection{Thermal parameter $\rho c k$ : material properties}

The TSP paint consists of three layers sprayed onto an aluminum substrate: the bottom primer layer, a screen layer and finally the active layer consisting of the polymer (here PU) with its embedded luminophores (Eu complex). Sottong (DLR Cologne, private communication (2015)) carried out material and thermal analyses of the aluminum substrate and these three layers. Each layer thickness and thence its density were determined; Fig. 3.13 shows a scanning electron microscope (SEM) image of a sectional cut through the aluminum substrate and its paint coatings, showing the thicknesses of the three layers and the structure of their surfaces. Heat conductance coefficients $k$ for the layers and substrate at temperatures from $25^{\circ} \mathrm{C}$ to $50{ }^{\circ} \mathrm{C}$ were also measured; these values for $k$ (in $\mathrm{Wm}^{-1} \mathrm{~K}^{-1}$ ) are plotted as a function of temperature in Fig. 3.14. The coefficient $k$ for aluminum is much larger than that for the active layer, by a factor of about 100 . However, in the previous estimate of $k$ in Table 3.1, the factor was more like $600(\approx 110 / 0.18)$, which is obviously a huge over-estimate, based on these new measurement results. The conclusion remains the same, however: the main driving force for the different response of the Medtherm and TSP paint to an externally applied heat load is the large difference in $k$. The largest temperature dependence is 


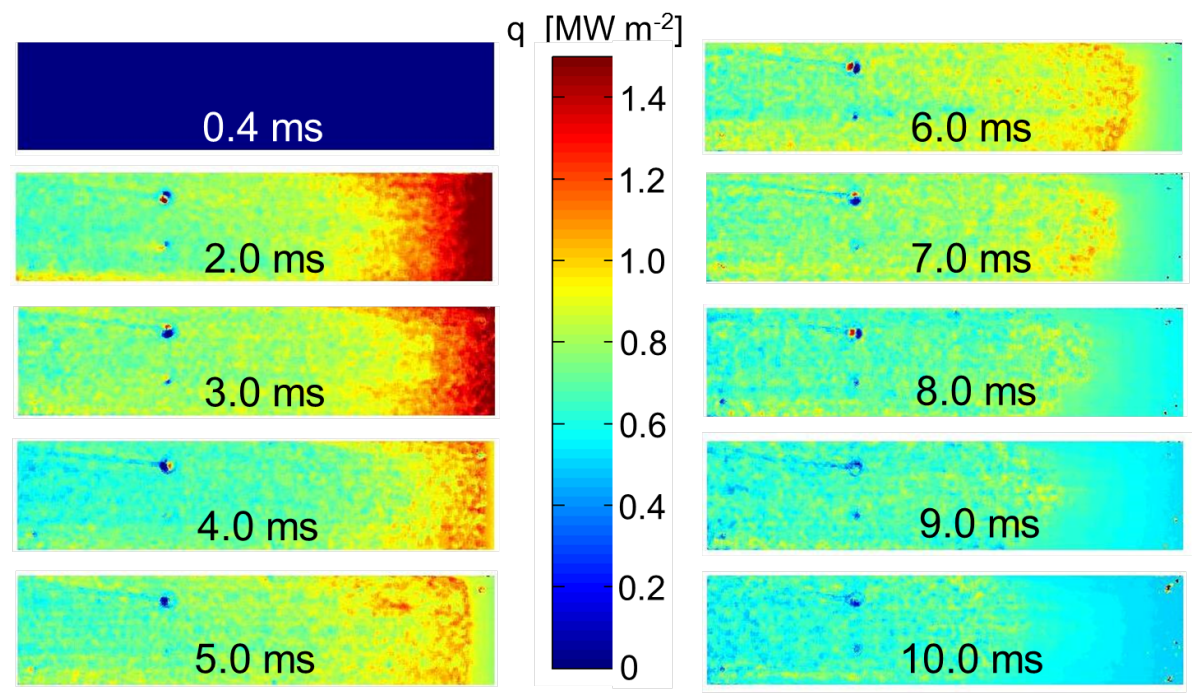

Figure 3.11: Heat loads from TSP measurement (2D images) over the whole model surface for 10 different times during the HEG run. (Flow in these images is from right to left)

shown by the innermost primer and the active layers, where $\mathrm{k}$ drops by about $15 \%$ from $25^{\circ} \mathrm{C}$ to $50^{\circ} \mathrm{C}$. For the middle screen layer the drop is only about $7 \%$. One can assume (and parametric tests here have shown) that for these short test times $(<10 \mathrm{~ms})$ mainly (but not solely) the material properties of the outer active layer play the more significant role in influencing the heat transfer process.

Finally, using the layer properties determined by Sottong, one can obtain a value of $\rho c k$ and compare it with that determined by the Medtherm insitu calibration of the TSP paint (which was $\rho c k_{\text {insitu }}=4.5 \times 10^{6} \mathrm{~kg}^{2} \mathrm{~K}^{-2} \mathrm{~s}^{-5}$ - see before). Recall that this is different than the approach used by (Nakakita et al. 2003), where very thin paint layers were used (see Sect. 3.2). (For reference, recall that $\rho c k_{\text {Medtherm }}=7.95 \times 10^{7} \mathrm{~kg}^{2} \mathrm{~K}^{-2} \mathrm{~s}^{-5}$ ). Hence, over the temperature range $25^{\circ} \mathrm{C}$ to $50^{\circ} \mathrm{C}$, it is estimated that the assumption of a constant (viz. average) value of $k$ would also lead to an uncertainty (inaccuracy) in the calculated heat loads of about $\pm 5 \%$ (recall: $(\rho c k)^{1 / 2}$, and not $\rho c k$, enters into Eq. 3.3. Sottong measured a constant value of $c=930 \pm 45 \mathrm{~m}^{2} \mathrm{~s}^{-2} \mathrm{~K}^{-1}$ for all layers and over a temperature range $0{ }^{\circ} \mathrm{C}$ to $50{ }^{\circ} \mathrm{C}$. Taking the TSP sample values supplied by Sottong: $\rho=1330 \mathrm{kgm}^{-3}, c=930 \pm 45 \mathrm{~m}^{2} \mathrm{~s}^{-2} \mathrm{~K}^{-1}$ and $k=2.5 \pm 0.2 \mathrm{Wm}^{-1} \mathrm{~K}^{-1}$ (this value being for just the active layer), one obtains $\rho c k_{\text {mat }}=3.1( \pm 0.3) \times 10^{6} \mathrm{~kg}^{2} \mathrm{~s}^{-5} \mathrm{~K}^{-2}$, based just on the material properties. $\rho c k_{\text {insitu }}$ and $\rho c k_{\text {mat }}$ differ by about $30 \%$. If one assumes that the second screen layer also plays some role in the heat transfer process, this would lead to larger effective values of $k$, lying somewhere in the range $2.3 \mathrm{Wm}^{-1} \mathrm{~K}^{-1}$ (active layer) and $6.1 \mathrm{Wm}^{-1} \mathrm{~K}^{-1}$ (screen), with the subsequent $\rho c k_{\text {mat }}$ then lying between 3.1 and $8.2 \mathrm{~kg}^{2} \mathrm{~s}^{-5} \mathrm{~K}^{-2} . \rho c k_{\text {insitu }}$ is right in the middle of this range. $c$ was measured to be temperature-independent in the range $25^{\circ} \mathrm{C}$ to $50^{\circ} \mathrm{C}$. However, $c$ was measured for the whole sample, and not for the individual layers, which makes it difficult to assess how this simplification may affect the appropriate value of $c$ for the active layer. In conclusion, then, given these uncertainties in the material properties, and based on present available information, one 


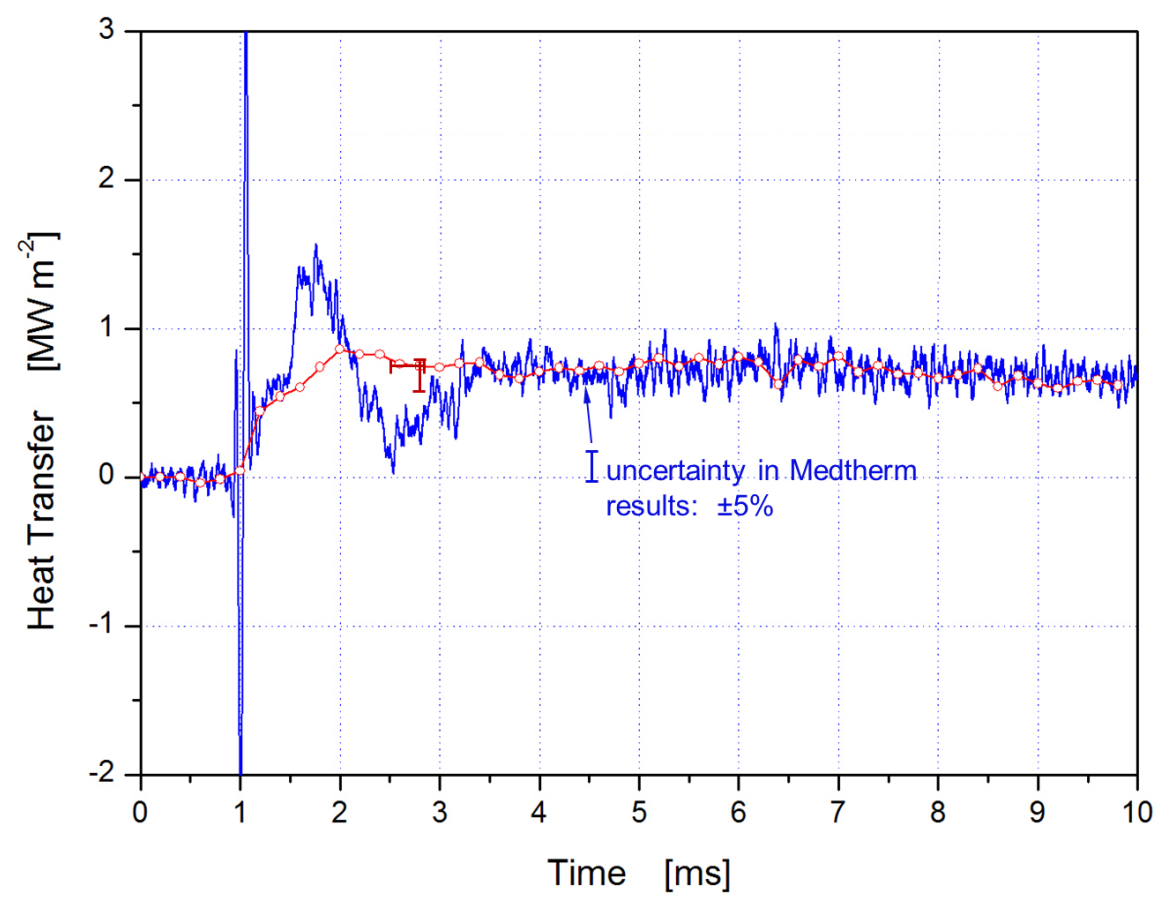

Figure 3.12: Comparison of heat load on the ramp from TSP (in the interrogation area) and from Medtherm vs. time. The $\rho c k$ value for TSP was obtained by an insitu calibration using the Medtherm result - see text

can nevertheless state that the thermal parameters based on the insitu calibration with the Medtherm are the most reliable. Values based on material properties have a large degree of uncertainty, which adds support and weight to the insitu method of calibration adopted here.

\subsubsection{The interrogation area}

As mentioned before, an interrogation area of $15 \times 15$ pixels (corresponding to about $2.5 \mathrm{~mm} \times$ $2.5 \mathrm{~mm}$ ) had been used to compare TSP and Medtherm values - see Fig. 3.5. This size choice represents a trade-off between two counteracting aspects: the area must be large enough to deliver good statistical dynamics (especially at later times where the signals are quite low - see Fig. 3.5) on the one hand, but, on the other, small enough to avoid too much spatial smearing (the Medtherm has a $1.6 \mathrm{~mm}$ diameter, corresponding to an area of about $2.0 \mathrm{~mm}^{2}$ ). An analysis of the interrogation area for all 50 images led to a maximum value of relative error (based on the mean and standard deviation) of $2 \%$ for the intensities (Figs. 3.5 and 3.8), and for temperatures of $\pm 2 \mathrm{~K}$ at $292 \mathrm{~K}$ and $\pm 4 \mathrm{~K}$ at $340 \mathrm{~K}$ (see Fig. 3.10); similar uncertainties are to be expected for the heat loads. These error estimates are based solely on the statistics of counts in the interrogation region. They do not represent the total error/uncertainty - see later. 


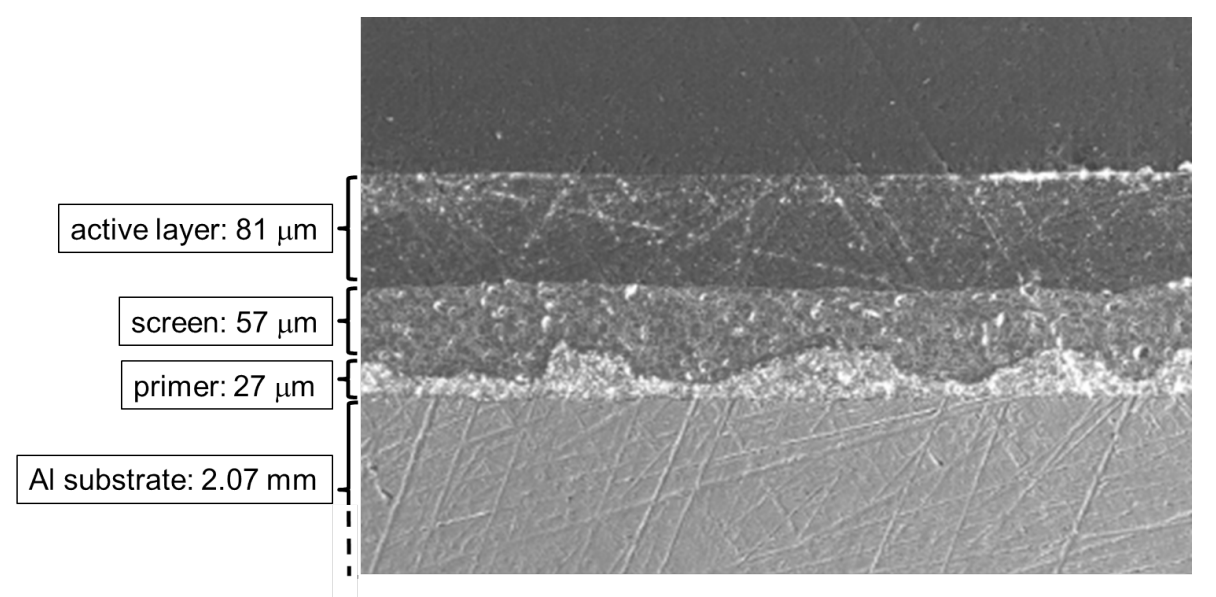

Figure 3.13: SEM (scanning electron microscope) image of cross section through various TSP paint layers, used to determine layer thicknesses (and thence density). Source: see text

\subsubsection{Correction for time response of the paint}

This is potentially a larger source of uncertainty in the final heat load values, influencing both the time and amplitude axes. After excitation by LED light of the Eu complexes in the OV322 paint to an excited electronic state, these Eu molecules relax back to their ground state with a characteristic luminescence relaxation time $\tau$. Generally one assumes a mono-exponential decay (Liu and Sullivan, 2005), so that $\tau$ represents just one simple decay process characterized by just this one constant. The decay process is temperature-dependent, with temperature influencing the rate of thermal quenching of the excited molecular state: use is in fact made of this phenomenon in the TSP lifetime method, where measurement of intensity decay rather than relative intensity is used by Ondrus et al. (2015). They measured $\tau$ as a function of temperature and pressure for the OV322 paint - see Fig. 3.15 .

Since pressures in the HEG run were around $100 \mathrm{kPa}$ (see Fig. 3.7 earlier), $\tau$ is then seen to be about $330 \mu$ s and $200 \mu \mathrm{s}$ at $25^{\circ} \mathrm{C}$ and $50^{\circ} \mathrm{C}$, respectively. These are long times, of duration similar to that of the camera exposure time $\left(t_{E}=200 \mu \mathrm{s}\right)$ in the HEG measurement with $\mathrm{CW}$ LED excitation. Hence, for any given exposure window (image), the measured signal is made up of components from fluorescence excited in this window plus decaying fluorescence excited in previous windows. This leads to a time smearing of the results over some windows, and perhaps also to an influence on the measured intensity in the window (especially so if the fluorescence intensity changes significantly from one window to the next). The following is an assessment of the influence of this effect on ensuing uncertainties in both time and amplitude, and will be carried out based on the following assumptions?

1. Fluorescence decay time $\tau \approx 400 \mu \mathrm{s}\left(=2 t_{E}\right)$;

\footnotetext{
${ }^{3}$ It should be noted, that these assumptions were made in order to assess the measurement uncertainty for the described measurement case. It is not meant to be a general and rigorous analysis for all possible measurement conditions.
} 


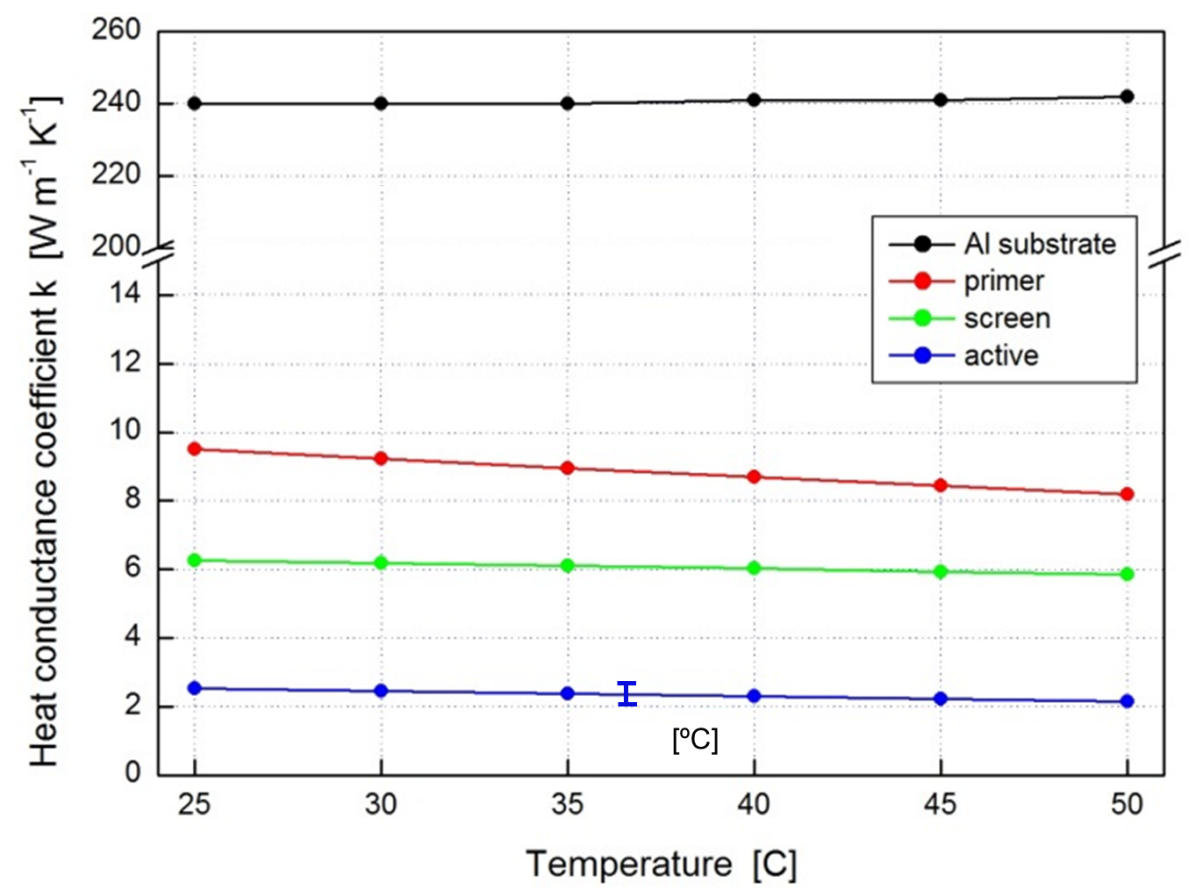

Figure 3.14: Measured heat conduction coefficients $k$ as a function of temperature for the three layers making up the TSP paint (error bar for active layer shown). Note break in the ordinate scale. Source: see text

2. Decay over several windows is given by just the one decay constant;

3. Excitation is via a very short single LED pulse at the start of each window;

4. Only three contiguous windows are considered;

5. This analysis is considered to apply for a large number of single pulse excitations over the whole window (viz. simulating $\mathrm{CW}$ excitation);

6. Areas $A$ under the curves (see Fig. 3.16) are assumed to scale with initial intensities (see text).

The sketch of fluorescence decay curves in Fig. 3.16 (note: ordinates are not drawn to scale) show in the abscissa adjacent exposure time windows of width $t_{E}$ (where $t_{E}=200 \mu \mathrm{s}$ ). The signal measured by the camera in window $n$ (in green), is made up of the sum of contributions from excitation in windows $(n-2),(n-1)$ and $n$ itself, and will now be considered. For initial simplification, it is assumed that excitation in each of the windows $(n-2),(n-1)$ and $n$ occurs at the beginning (e.g. by a very short LED pulse), with ensuing fluorescence decay over three subsequent windows to the respective levels $1 / e^{3 / 2}, 1 / e^{1 / 2}$ and $1 / e$ of the initial fluorescence $I_{i}$. For example, fluorescence from window $(n-2)$ has fallen to a value of $1 / e^{3 / 2} \approx 22 \%$ of its original value at the end of window $n$; contributions from even earlier windows are not included in this assessment. Furthermore, for simplification, it has been assumed that $\tau \approx 2 t_{E}$ (this is 


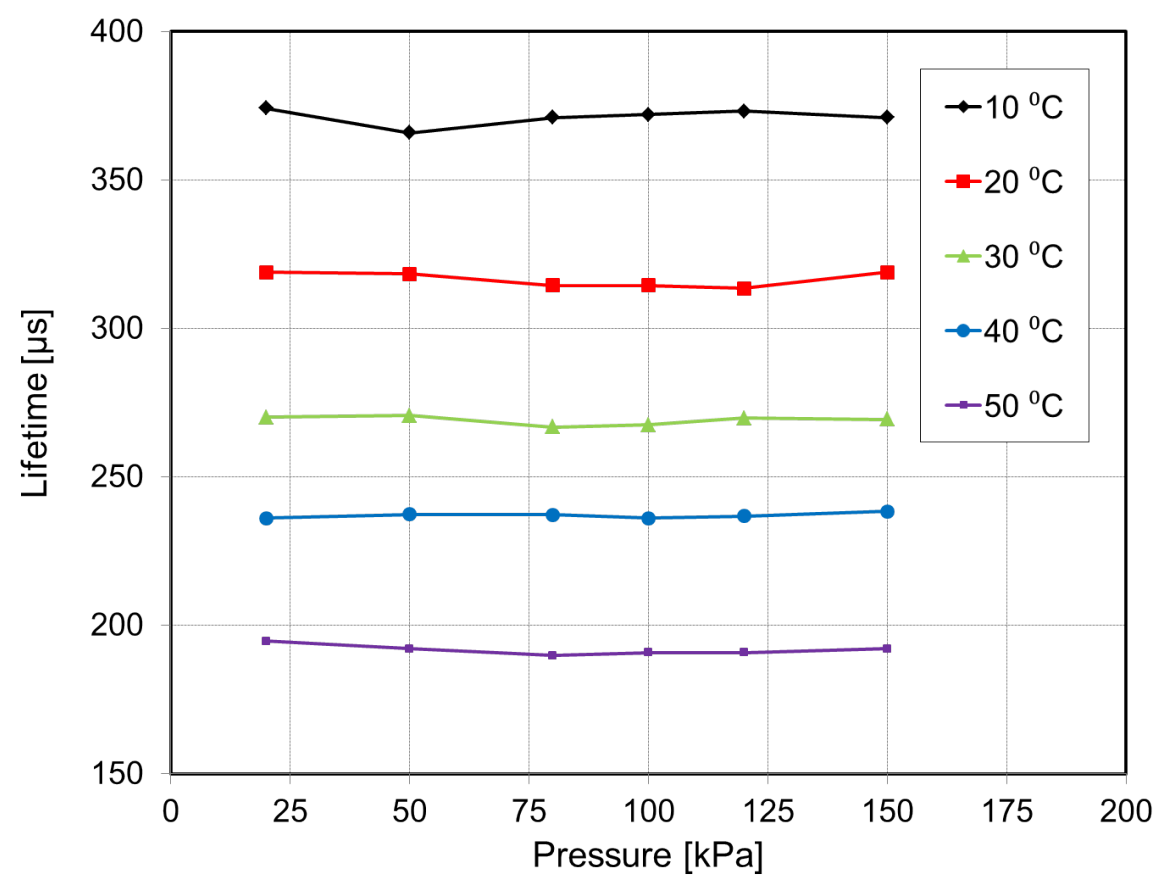

Figure 3.15: Luminescence lifetimes $\tau$ of OV322 paint as a function of temperature and pressure $p$ (see text). (Source: Ondrus et al. (2015))

approximately correct at the early times). The intensity measured by the camera in window $n$ is then $A(n-2)_{3}+A(n-1)_{2}+A n_{1}$ (see Fig. 3.16, namely:

$$
I_{t o t}=I_{n-2} \int_{2 t_{E}}^{3 t_{E}} e^{\left(-t / 2 t_{E}\right)} d t+I_{n-1} \int_{t_{E}}^{2 t_{E}} e^{\left(-t / 2 t_{E}\right)} d t+I_{n} \int_{0}^{t_{E}} e^{\left(-t / 2 t_{E}\right)} d t
$$

where $I_{n-2}, I_{n-1}$ and $I_{n}$ are the initial fluorescence intensities in their respective windows. For Case 1 in Fig. 3.16, it has been assumed that $I=I_{n-2}=I_{n-1}=I_{n}$, so that Eq. 3.4 simplifies to:

$$
\begin{aligned}
& I_{t o t}=I\left[\int_{2 t_{E}}^{3 t_{E}} e^{\left(-t / 2 t_{E}\right)} d t+I_{n-1} \int_{t_{E}}^{2 t_{E}} e^{\left(-t / 2 t_{E}\right)} d t+I_{n} \int_{0}^{t_{E}} e^{\left(-t / 2 t_{E}\right)} d t\right] \\
& I \int_{0}^{3 t_{E}} e^{\left(-t / 2 t_{E}\right)} d t
\end{aligned}
$$

Hence, from Fig. 3.16 ,

$$
\begin{aligned}
& A n_{3}=I_{n-2}\left[\int_{2 t_{E}}^{3 t_{E}} e^{\left(-t / 2 t_{E}\right)} d t\right] \equiv A(n-2)_{3} \\
& A n_{3}=I_{n-2}\left[\int_{t_{E}}^{2 t_{E}} e^{\left(-t / 2 t_{E}\right)} d t\right] \equiv A(n-2)_{2}
\end{aligned}
$$

In other words, the measured signal would be the same as that which arises from capturing the fluorescence from just the one excitation at the start of window $\mathrm{n}$ for the three following windows $n$, $n+1$ and $n+2$. In Fig. 3.16 Case 1 this is shown pictorially: $A n_{1}+A n_{2}+A n_{3}=A n_{1}+A(n-1)_{2}+$ $A(n-2)_{3}$. Case 1 is the "trivial" case and applies only for equal initial fluorescence intensities. In Case 2 these intensities are not equal, but, for the measurements here, falling, as shown in Fig. 3.16 


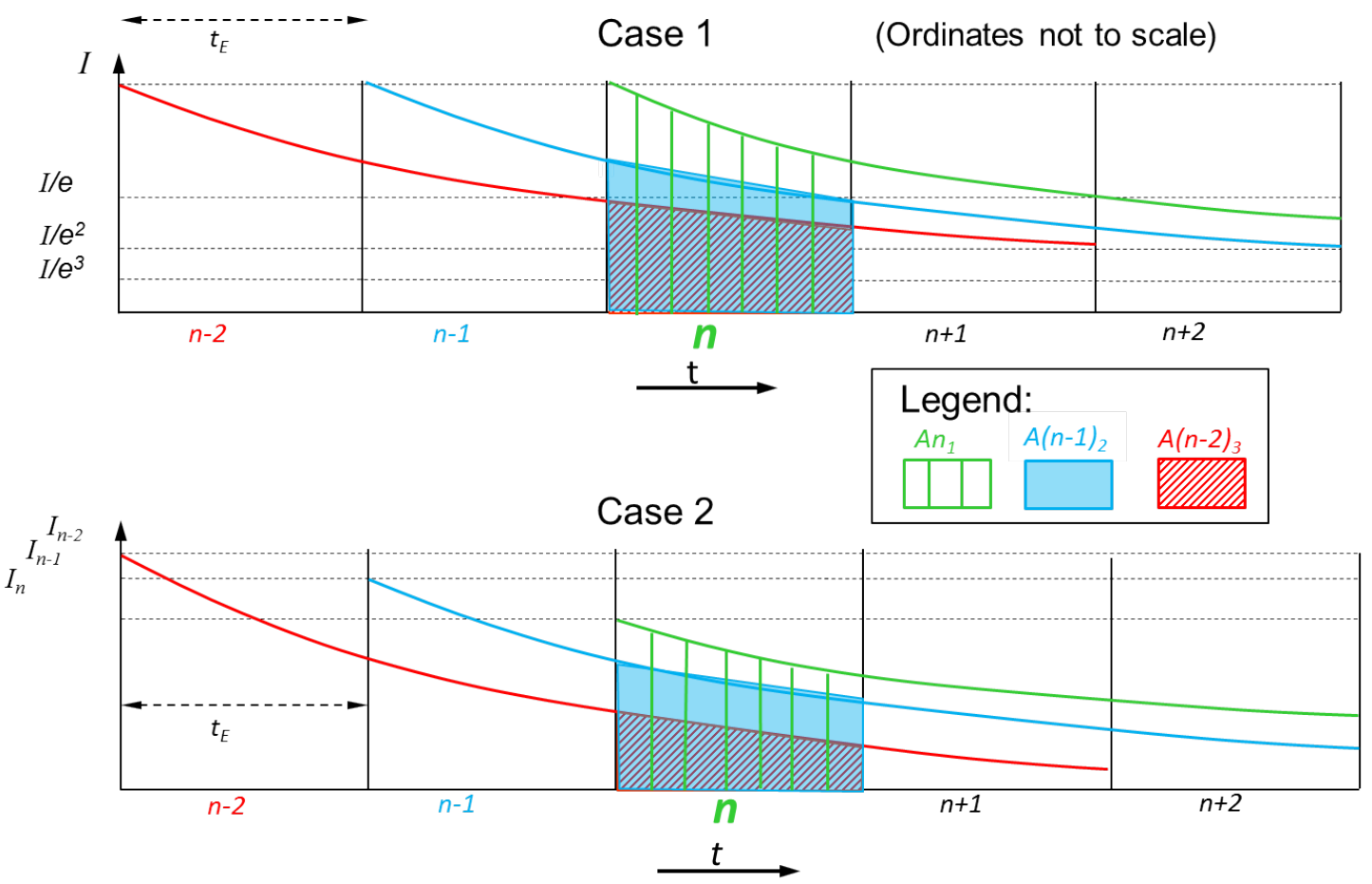

Figure 3.16: Fluorescence decay curves over contiguous windows assuming single short pulse excitation at the beginning of each window. Constant (Case 1) and changing (Case 2) initial fluorescence intensities for the 3 windows are considered. (See text.) The areas $A$ pertain to those under the corresponding curves for fluorescence decay from windows $(n-2),(n-1)$ and $n$

Case 2. (Obviously increasing temperature over time leads to decreasing fluorescence intensity.) Hence the contributions from windows $n-1$ and $n-2$ to intensity measured by the camera in window $n$ are larger, leading to a measured intensity which is too high: $A n_{3}<A(n-2)_{3}$ and $A n_{2}<A(n-1)_{2}$. The influence of this effect on the measurement uncertainty in window $\mathrm{n}$ will now be assessed, based on the measured differences between the intensities $I_{n-2}, I_{n-1}$ and $I_{n}$, as given by the results shown earlier in Fig. 3.5. here one sees that intensities drop by about $8 \%$ per window over the first three windows after gas arrival, where this drop is largest: $I_{n-2} / I_{n-1} \approx I_{n-1} / I_{n} \approx 0.92$. Assuming that the relative areas $A(n-2)_{3} / A n_{3}$ and $A(n-1)_{2} / A n_{2}$ (see Fig. 3.16 Case 2) scale with the initial intensities, namely:

$$
\begin{aligned}
& \frac{A(n-2)_{3}}{A n_{3}}=\frac{I_{n-2}}{I_{n}}=0.92^{2} \\
& \frac{A(n-1)_{2}}{A n_{2}}=\frac{I_{n-2}}{I_{n}}=0.92
\end{aligned}
$$


the total intensity $I_{t o t}$ measured in window $n$ then becomes:

$$
\begin{aligned}
I_{t o t} & =A n_{1}+A(n-1)_{2}+A(n-2)_{3} \\
& =A n_{1}+A n_{2} \cdot \frac{I_{n-1}}{I_{n}}+A n_{3} \cdot \frac{I_{n-2}}{I_{n}} \\
& =A n_{1}+0.92 \cdot A n_{2}+0.92^{2} \cdot A n_{3}
\end{aligned}
$$

Now evaluate the integrals $A n_{1}, A n_{2}$ and $A n_{3}$ to get:

$$
A n_{1}: A n_{2}: A n_{3}=1: \frac{1}{e^{1 / 2}}: \frac{1}{e^{3 / 2}} \approx 100: 60: 36
$$

The discrepancies in the total signal $A n_{1}+A n_{2}+A n_{3}$ arise from the abovementioned differences for $A n_{2}$ vs. $A(n-1)_{2}$ and for $A n_{3}$ vs. $A(n-2)_{3}$, which correspond to, respectively, $(1-0.92)=$ $0.08=8 \%$ and $(1-0.922)=0.15=15 \%$. This leads to a total signal of $100+60+36=196$, while the correction factor (or uncertainties) amount to $60 \cdot(-0.08)+36 \cdot(-0.15)=-10$. The relative uncertainty is therefore about $-10 / 196=-0.05=-5 \%$. This is strictly not an error, but corresponds to a systematic discrepancy or a correction factor. Note that the sign is negative (since the intensity is dropping). The above discussion and estimations were based on an assumption of single short pulse LED excitation at the beginning of each window. However, CW LED excitation was used in the experiment. If one considers the $\mathrm{CW}$ excitation over a window to be made up of an infinite (viz. a large number) of single pulse excitations, then the conclusions reached here should still be valid, since each single pulse excitation at times within the window would behave and be evaluated in a similar way as above. The total uncertainty in amplitude (intensity), based only on those issues discussed in the preceding subsections, is hence estimated to be $2 \%$ and $-7 \%$. The uncertainty in the time domain, based on the model calculations discussed above, is $-2 t_{E}$, i.e. about $-0.4 \mathrm{~ms}$. These errors/uncertainties are included in the respective plots shown earlier - note they are asymmetrical.

\subsubsection{Normalized TSP and Medtherm temperatures}

Finally, a check on the performance of the TSP paint is to plot normalized temperature $T$ vs. time $t$, and assume that $T=f\left(t^{1 / 2}\right)$, or $T_{2}=f(t)$ - as suggested from Eq. 3.3 . If the behaviour of TSP (especially its time response) were to have a strong effect, one would expect the plots for TSP and Medtherm to show different trends over time. Fig. 3.17 shows a plot of $\left[\left(T_{t}-T_{0}\right) /\left(T_{\max }-T_{0}\right)\right]^{2}$ vs. $t$, where $T_{0}=$ temperature before gas arrival, $T_{\max }=$ temperature at $10 \mathrm{~ms}$ and $T_{t}=$ temperature at time $t$. Apart from the bumps in the Medtherm plot at early times, both plots show very similar behaviour, demonstrating that TSP, in spite of its larger time response (compared with Medtherm), can still deliver meaningful results of temperatures and thence of heat load.

\subsection{Conclusions}

This work has demonstrated the usability of TSP to measure temperatures on a surface in a hypersonic, short-duration flow, and therefrom to calculate the heat load, making use of those 


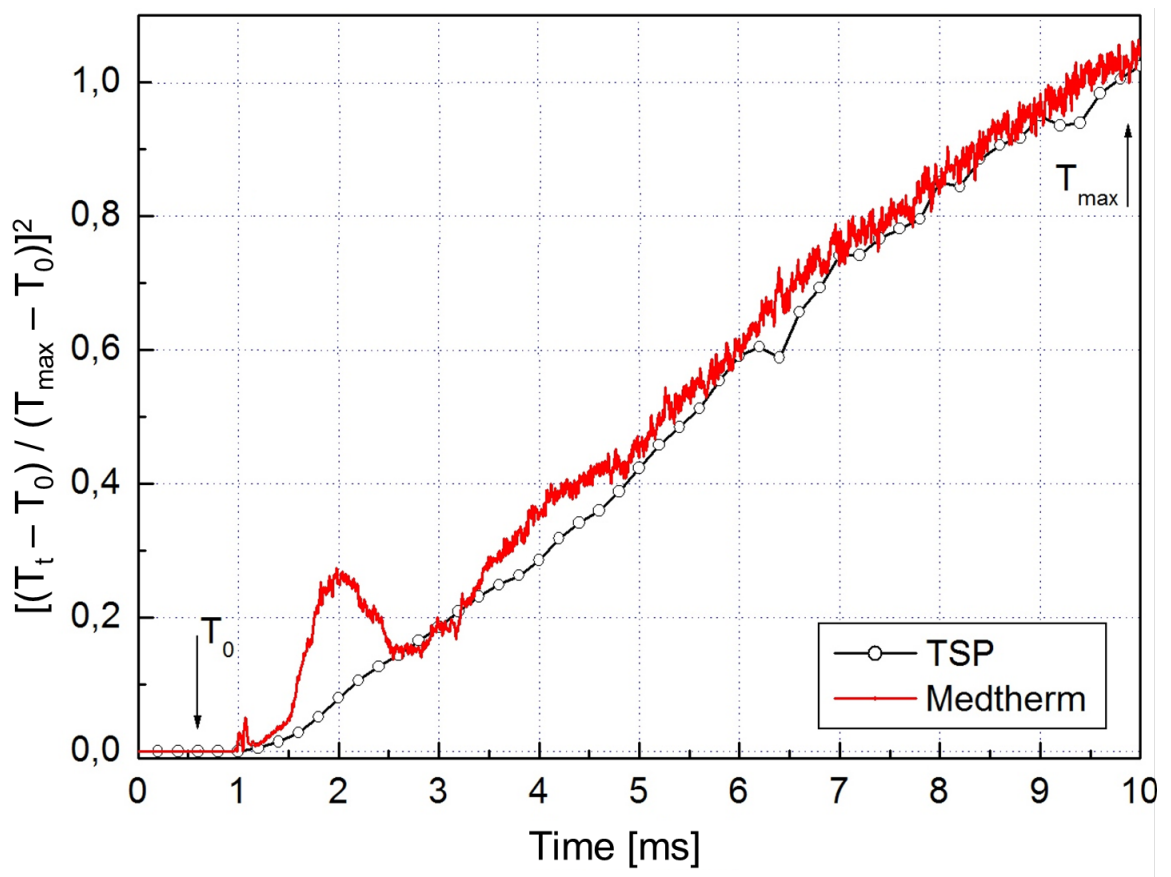

Figure 3.17: TSP and Medtherm temperatures T vs. time $t$, plotted normalized to minimum and maximum values, and assuming $T=f\left(t^{1 / 2}\right)$ (viz. $T_{2}=f(t)$ ): see Sect. 3.6.4.4. $T_{0}$ is the temperature before gas arrival; $T_{\max }$ is the temperature at $10 \mathrm{~ms}$. For error/uncertainty estimate: see Fig. 3.12

assumptions generally applied to these types of flows. Both temperature and heat load results on the surface of a ramp model placed in a Mach $M=7.4$ HEG flow were shown. A Medtherm coaxial thermocouple situated at one position on the model was used to calibrate the TSP paint (viz. to obtain the thermal parameter $\rho c k$ for the paint). An average heat load in the interrogation region of $0.73 \pm 0.07 \mathrm{MWm}^{-2}$ has been measured. A further more detailed discussion of sources of error and uncertainty in the final results was given.

\section{Acknowledgements}

The authors are grateful to the following for their assistance: Werner Sachs (DLR, now retired) for help in data processing using the DLR in-house code nToPas; Marco Costantini (DLR Göttingen) for invaluable contributions to determining the TSP paint material properties; Reinhard Sottong (DLR Cologne) for carrying out measurements of material properties on samples of paint; Jan Martinez Schramm (DLR Göttingen) for test preparation support; Vlado Ondrus (University of Hohenheim) for development of the paint OV322; Uwe Fey (DLR Göttingen) for construction of the LED multi-lens array. 


\section{Mach number effects in the Cryogenic Ludwieg-Tube Göttingen (Risius et al., 2018a)}

Citation and credit: Reprinted with permission from New Results in Numerical and Experimental Fluid Mechanics XI. Notes on Numerical Fluid Mechanics and Multidisciplinary Design, vol 136, Springer, Cham, 2018, doi:10.1007/978-3-319-64519-3_28, Copyright 2018, Springer-Verlag GmbH Germany

Reference: Risius et al. (2018a)

Title: "Experimental investigation of Mach number and pressure gradient effects on boundarylayer transition in two-dimensional flow"

Authors: Steffen Risius, Marco Costantini, Stefan Hein, Stefan Koch and Christian Klein

\section{Contributions of the first author:}

- improved the TSP measurement system in order to carry out TSP measurements with higher spatial and temporal resolution in the Cryogenic Ludwieg-Tube Göttingen (DNW-KRG)

- carried out the model assembly and installation in DNW-KRG

- automated the data acquisition and evaluation of temperature sensors, pressure transducers and TSP result images

- developed the test matrices, organized and carried out the measurement campaigns

- checked the results for consistency throughout the measurement campaigns

- investigated the separation region at the trailing edge of the PaLASTra model and reduced its size by installation of an additional aft part

- showed the impact of separation-induced pressure fluctuations on the location of laminarturbulent transition

- developed a correction method to calculate adiabatic transition Reynolds numbers

- re-analyzed the turbulence spectrum of DNW-KRG to quantify its influence on laminarturbulent transition

- quantified the surface-pressure gradient by the Hartree parameter and shape factor

- applied a TSP calibration to retrieve quantitative temperature distributions in streamwise direction

- carried out boundary-layer calculations and compressible and incompressible linear stability 
analysis

- analyzed the results which revealed the connection between the turbulence level and the transition locations

- quantified pressure gradient, Mach number and unit Reynolds number effects on laminarturbulent transition

- compared results by compressible and incompressible critical $N$-factor calculations and showed their consistency with earlier measurements

- made the first and final version of all figures

- wrote the first and final version of the manuscript

- carried out the correspondence with the editor and implemented the corrections suggested by the referees

\subsection{Abstract}

The influence of Mach number $(M=0.35$ to 0.65$)$, chord Reynolds number $\left(R e_{c}=6 \times 10^{6}\right.$ to $\left.10 \times 10^{6}\right)$ and pressure gradient $\left(d c_{p} / d x=-0.6\right.$ to $\left.0.07 \mathrm{~m}^{-1}\right)$ on laminar-turbulent boundary layer transition was experimentally investigated in the Cryogenic Ludwieg-Tube Göttingen (DNW-KRG). For this investigation the existing two-dimensional wind tunnel model, PaLASTra, which offers a quasi-uniform streamwise pressure gradient, was modified in order to reduce the size of the flow separation at its trailing edge. The streamwise temperature distribution and the location of laminar-turbulent transition were measured by means of temperature-sensitive paint (TSP). It was found that the transition Reynolds number exhibits a linear dependence on the pressure gradient, characterized by the Hartree parameter, and that an increasing Mach number leads to a linear decrease of the transition Reynolds number. The latter effect is likely due to an increase of the total pressure turbulence level with Mach number in DNW-KRG. The measured pressure and temperature distributions served as input for boundary layer calculations and linear-stability analysis. $\mathrm{N}$-factors were calculated according to compressible and incompressible stability theory. At zero pressure gradient a critical $N$-factor of approximately 9.5 and 9.0 was found for incompressible and compressible calculations, respectively.

\subsection{Introduction}

Drag reduction by means of natural laminar flow technology is a promising way to reduce wall shear stress, which can reduce fuel consumption of commercial aircraft significantly (Schrauf, 2005). Tollmien-Schlichting (T-S) waves are the dominating mechanism leading to 'natural' transition of boundary layers developing on smooth two-dimensional surfaces in low free-stream disturbance environments (Schlichting and Gersten, 2000). At these conditions the linear stability 


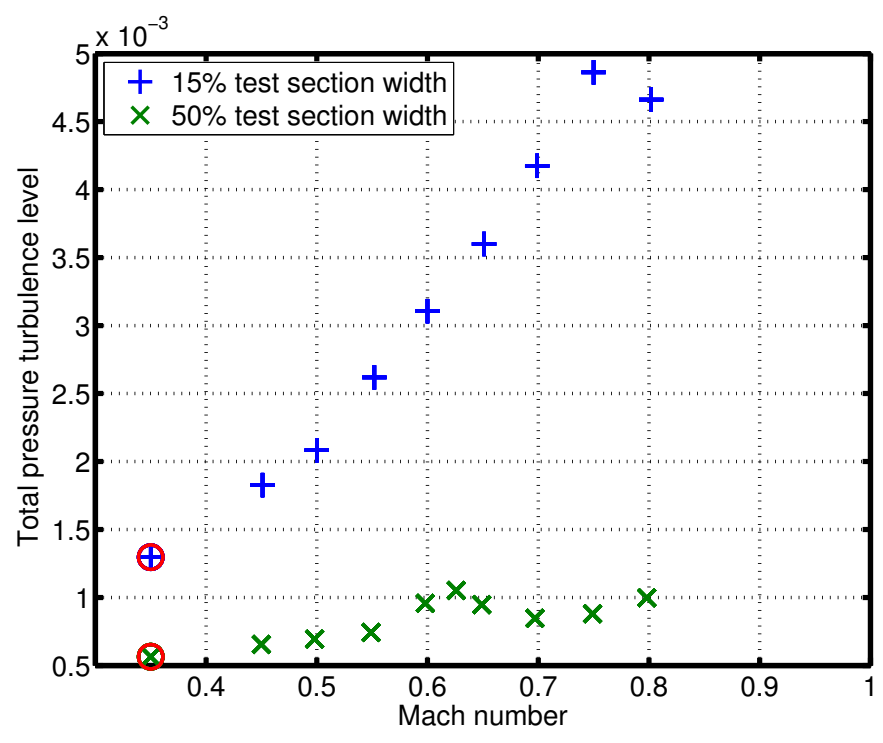

Figure 4.1: Total pressure turbulence level as a function of $M$ in DNW-KRG at $15 \%$ and $50 \%$ test section width. Encircled data points were extrapolated from measured data. Based on Fig. 4.35 of Koch (2004)

theory (Schlichting and Gersten, 2000), in particular the $e^{N}$-method, predicts a stabilizing effect of compressibility on boundary layer transition (Arnal and Vermeersch, 2011). However, there is only limited experimental verification of this effect for large subsonic Mach numbers. In this work we show a systematic experimental investigation of pressure gradients, Mach numbers and chord Reynolds number effects on boundary layer transition.

\subsection{Experimental Setup and Test Conditions}

The experiments were performed in the low-turbulence, based on mass flux $\left(T u_{\rho u} \approx 0.6 \times 10^{-3}\right)$, Cryogenic Ludwieg-Tube Göttingen (DNW-KRG) (Koch, 2004; Rosemann, 1997), a wind tunnel operated with gaseous nitrogen as working fluid. By expansion of the working fluid a temperature difference between flow and model surface occurs during a run. Therefore, the model surface temperature, $T_{w}$, is generally higher than the adiabatic wall temperature, $T_{a w}$, which enhances boundary layer instability and can cause transition to occur further upstream than in the adiabatic case (Schlichting and Gersten, 2000). However, the influence of a non-adiabatic surface temperature on the transition Reynolds number can be corrected (Costantini et al., 2016a). For calculation of adiabatic transition Reynolds numbers, $R e_{x T \text {, aw }}$, we use the following approximation, based on a linear fit of the data from Costantini et al. (2016a), to correct the measured non-adiabatic transition Reynolds number 1 .

$$
\operatorname{Re}_{x T} / \operatorname{Re}_{x T, a w} \approx\left(T_{w} / T_{a w}\right)^{-4} \approx-3.455 \cdot T_{w} / T_{a w}+4.455
$$

\footnotetext{
${ }^{1}$ The term 'transition Reynolds number' will always refer to $R e_{x T, a w}$ in the following.
} 
The uncertainties in the inflow Mach and Reynolds numbers in the present work were within $M= \pm 0.002$ and $R e_{c}= \pm 0.05 \times 10^{6}$. In order to guarantee an interference free flow around the wind tunnel model the upper and lower walls of the test section were adapted (Rosemann, 1997). It is known that the total pressure turbulence level, $T u_{p 0}$, of DNW-KRG increases with Mach number Koch, 2004). At a fixed unit Reynolds number of $R e_{1} \approx 30 \times 10^{6} \mathrm{~m}^{-1}, T u_{p 0}$ increases by a factor of about 1.7 in the center of the test section from $T u_{p 0} \approx 0.06 \%$ at $M=0.45$ to $T u_{p 0} \approx 0.1 \%$ at $M=0.8$ (Fig. 4.1). It increases even more strongly by a factor of 2.5 in the vicinity of the wall (measured at $15 \%$ of the test section width): from $T u_{p 0} \approx 0.19 \%$ at $M=0.45$ to $T u_{p 0} \approx 0.49 \%$ at $M=0.8$ (Koch, 2004). This fact makes the comparison of transition Reynolds numbers measured at different Mach numbers challenging, as it will be discussed in Section 4.4 .

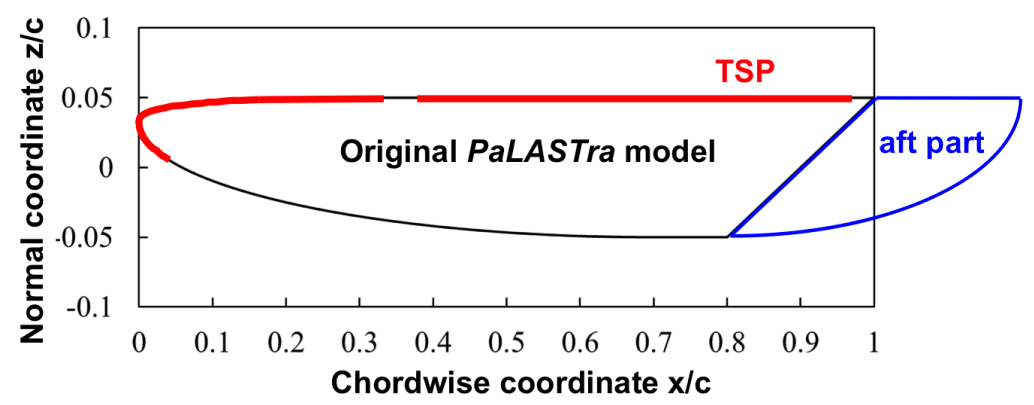

Figure 4.2: Side view of the original PaLASTra model with an additional aft part at the trailing edge and temperature-sensitive paint (TSP) on top (Costantini et al. 2015a, 2016a b). The original chord length of $c=0.2 \mathrm{~m}$ is used for normalization of the chordwise coordinate

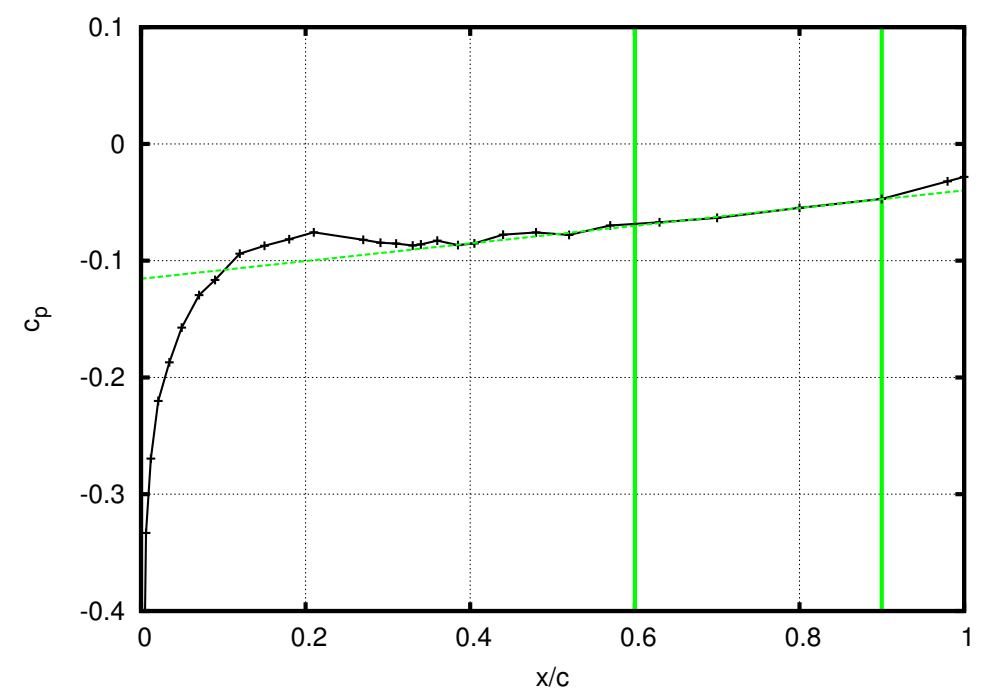

Figure 4.3: Distribution of pressure coefficient, $c_{p}$, on model upper side at $R e_{c}=6 \times 10^{6}$ and $M=0.65$. The continuous green lines at $x / c=0.6$ and 0.9 indicate the region where the pressure distribution was fitted linearly to evaluate $\beta_{H}$. The green dotted line visualizes the linear fit of the pressure distribution 
The wind tunnel model used for the present study was the two-dimensional PaLASTra model (Costantini et al., 2015a, 2016a b). On the lower surface of the original PaLASTra model an abrupt contour change was imposed at $x / c=80 \%$ (Fig. 4.2 to fix boundary layer separation (Costantini et al., 2016a). However, the large separation region, originating downstream of the original PaLASTra model, leads to strong pressure fluctuations (i.e. acoustic noise) inside the test section, which are likely to increase the initial amplitude of the disturbances within the laminar boundary layer and thus reduce the transition Reynolds number (Costantini et al., 2016a).

To reduce the separation region, an additional aft part was designed and attached to the original PaLASTra model as shown in Fig. 4.2. This modification of the model reduces the size of the separation region and the magnitude of the emitted pressure fluctuations. After modification the separation-induced pressure fluctuations were below the minimum measurable quantity of a Kulite pressure transducer (CCQ-093), which was operated at up to $200 \mathrm{kHz}$ and mounted flush with the test section side wall at a location upstream of the model.

The pressure distribution on the upper side of the modified PaLASTra model is shown in Fig. 4.3 , As the pressure gradient was essentially uniform on a large portion of the upper surface, an average pressure gradient was determined by fitting a linear function to the pressure distribution between $60 \%<x / c<90 \%$ In order to characterize the streamwise surface-pressure distribution, the average pressure gradient was then used to determine the Hartree parameter, $\beta_{H}$, with

$$
\beta_{H}=\left(\frac{1}{2}-\frac{1-c_{p}}{x\left(\partial c_{p} / \partial x\right)}\right)^{-1}
$$

The values of $x$ and $c_{p}$ at the center of the fitted region were used Meyer and Kleiser (1989).

Non-intrusive global measurement of the surface temperature distribution was carried out using a TSP (Ondrus et al., 2015). TSP formulation, surface quality, optical setup, acquisition and evaluation of the TSP images were the same as described in Costantini et al. (2016a). In order to determine the surface temperature distribution on the model upper side the TSP was calibrated in an external calibration chamber (Egami et al., 2007). Surface temperature distributions in the streamwise direction, extracted from the TSP data at five spanwise sections, are shown in Fig. 4.4 .

The influence of Mach number on boundary layer transition was investigated for three different Mach numbers $(M=0.35,0.50$ and 0.65$)$ at three different chord Reynolds numbers $\left(R e_{c}=6 \times 10^{6}\right.$, $8 \times 10^{6}$ and $10 \times 10^{6}$, based on a chord length of $c=0.2 \mathrm{~m}^{-1}$ in order to allow comparison with earlier results) for various pressure gradients. A systematic variation of the pressure gradient was possible by adjusting the angle-of-attack between $-3.5^{\circ}$ and $0^{\circ}$.

Boundary layer computations were performed using the boundary layer solver COCO (Schrauf, 1998), which was modified in order to incorporate not only the measured surface pressure but also the measured surface temperature distributions as inputs. Thus, it was accounted for the influence of the non-adiabatic surface temperature on the boundary layer. These computations were used to determine displacement $\left(\delta_{1}\right)$ and momentum thickness $\left(\delta_{2}\right)$, as well as the shape factor $H_{12}=\delta_{1} / \delta_{2}$, which was used to characterize the boundary layer velocity profile. In order to compare the critical

\footnotetext{
${ }^{2}$ For comparison, the pressure distribution was also fitted between $35 \%<x / c<90 \%$, leading to negligible differences in $\beta_{H}$ (not shown here).
} 

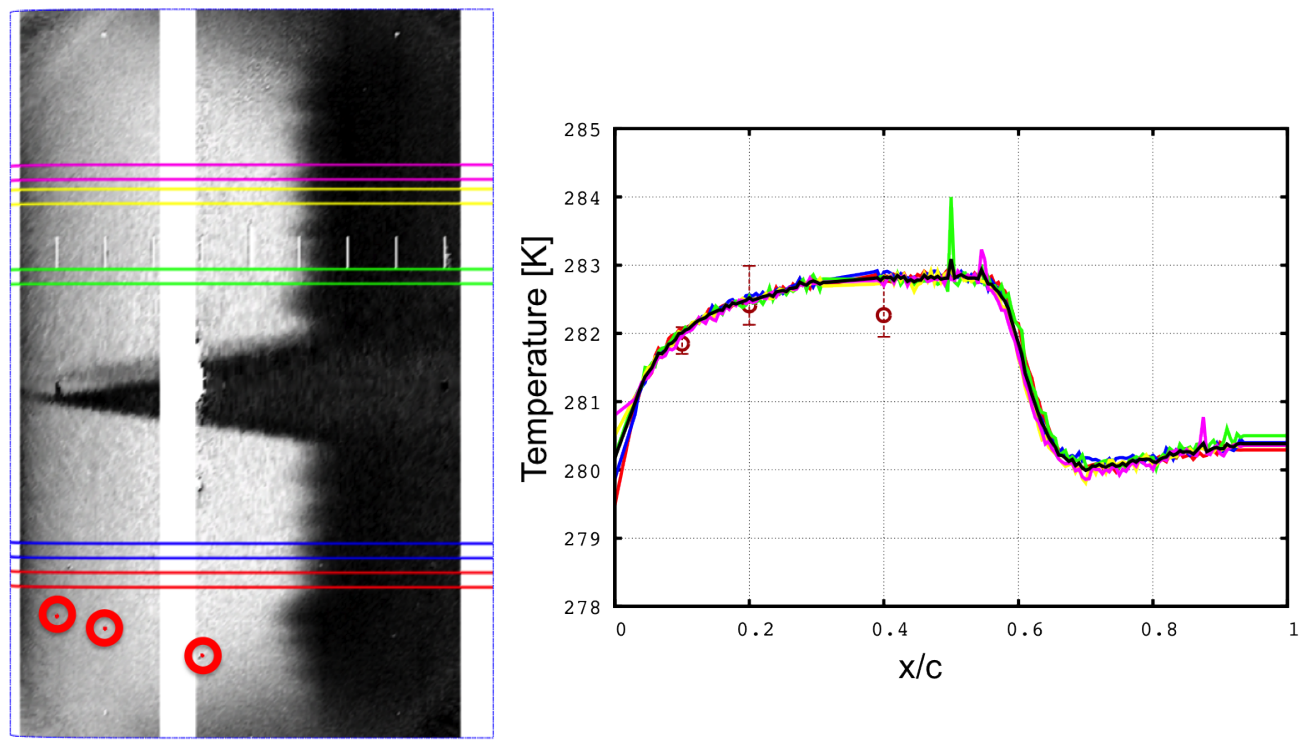

Figure 4.4: Left image: TSP result image at $\operatorname{Re}_{c}=6 \times 10^{6}, M=0.65$ and an angle of attack of $-1.5^{\circ}$, where bright and dark areas correspond to laminar and turbulent regions, respectively. The flow direction is from left to right. The whitened strips indicate metallic surfaces of the model where no paint had been applied. The two turbulent wedges in the mid-span domain are caused by pressure taps in the leading edge region. The red circles indicate the location of thermocouples embedded in the TSP (Costantini et al., 2016a). The horizontal lines indicate the location of temperature measurement by TSP. To increase the signal-to-noise ratio, the intensity values were averaged over a spanwise range, as indicated by means of two lines placed at the spanwise bounds of the evaluated range for each location (Costantini et al., 2016a).

Right image: Temperature distribution measured by TSP (colored lines, correspond to left image) and their average (black line) as a function of chordwise coordinate. The temperature information was inter- and extrapolated linearly in the whitened strips, where no paint had been applied. For comparison also the thermocouple measurements (red circles with error bars) are shown. The flow conditions are the same as in Fig. 4.3 and Fig. 4.5 


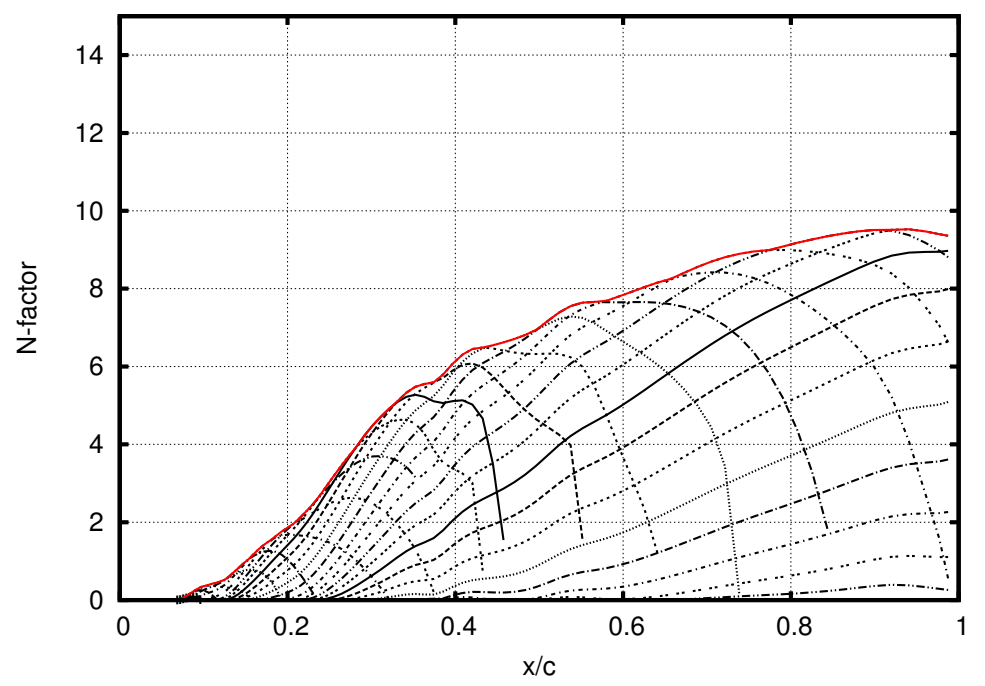

Figure 4.5: Amplification factors of Tollmien-Schlichting waves of compressible $N$-factor calculation as a function of normalized chordwise coordinate $x / c$, computed for the same test conditions as in Fig. 4.3 and Fig. 4.4. The lines correspond to different amplified frequencies ranging from $f_{\min }=5883 \mathrm{~Hz}$ to $f_{\max }=83000 \mathrm{~Hz}$. The critical $N$-factor was determined by finding the maximum $N$-factor (indicated by the red line) at the transition location measured with TSP

$N$-factors at different Mach numbers, the incompressible shape factor $H_{12 \text {,inc }}=\delta_{1, \text { inc }} / \delta_{2, \text { inc }}$ was used. Amplification rates of T-S waves for the computed boundary layer were determined by means of LILO (Schrauf, 2006). According to linear, local stability theory and the quasi-parallel flow assumption, both incompressible and compressible stability computations were carried out and their results were correlated with the measured transition location to assess critical $N$-factors (Fig. 4.5).

\subsection{Results}

\subsubsection{Mach number influence on the transition Reynolds number}

The adiabatic transition Reynolds number, $R e_{x T, a w}$, as a function of Hartree parameter, $\beta_{H}$, is shown in Fig. 4.6, for three different Mach numbers $(M=0.35,0.50$ and 0.65$)$, and a chord Reynolds number of $R e_{c}=6 \times 10^{6}$. A linear function $\operatorname{Re}_{x T, a w}\left(\beta_{H}\right)=a \cdot \beta_{H}+b$ with a slope, $a$, and an intercept, $b$, was fitted through each data set. The same data evaluation was carried out also for chord Reynolds numbers of $R e_{c}=8 \times 10^{6}$ and $10 \times 10^{6}$ (not displayed here). The intercepts, $b=\operatorname{Re}_{x T, a w}\left(\beta_{H}=0\right)$, of all data sets appear to be determined by Mach number and independent of the chord Reynolds number (Table 4.1). The mean and standard deviation of the intercepts were calculated separately for each Mach number (Table 4.1).

The mean intercepts, $\bar{b}$, as a function of $M$ can be fitted by the following linear function,

$$
\bar{b}=-7.53 \cdot M+6.73
$$




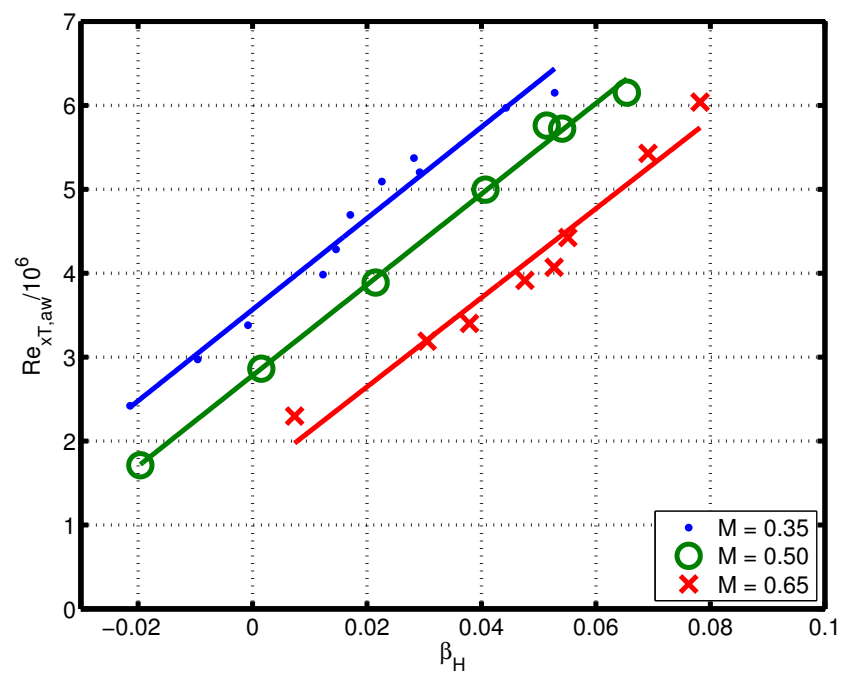

Figure 4.6: Transition Reynolds number, $R e_{x T, a w}$, as a function of Hartree parameter, $\beta_{H}$, for three different Mach numbers $M=0.35,0.50$ and 0.65 and a chord Reynolds number of $R e_{c}=6 \times 10^{6}$

Table 4.1: Intercepts, $b$, for each chord Reynolds number and Mach number as well as their mean, $\bar{b}$, and standard deviation in units of $10^{6}$. For each intercept the coefficient of determination of the linear fit, $R^{2}$, is given in brackets

\begin{tabular}{llll}
\hline & $M=0.35$ & $M=0.50$ & $M=0.65$ \\
\hline$R e_{c}=6 \times 10^{6}$ & $3.57(0.974)$ & $2.78(0.989)$ & $1.59(0.994)$ \\
$R e_{c}=8 \times 10^{6}$ & $3.96(0.996)$ & $3.42(0.977)$ & $1.47(0.996)$ \\
$R e_{c}=10 \times 10^{6}$ & $4.45(0.958)$ & $3.25(0.996)$ & $2.14(0.998)$ \\
\hline Mean & 3.99 & 3.15 & 1.73 \\
std. dev. & 0.44 & 0.33 & 0.36 \\
\hline
\end{tabular}


Table 4.2: Total pressure turbulence level, $T u_{p 0}$; normalized and inverted total pressure turbulence level $1 / \hat{T} u_{p 0}$; intercepts $b$; and normalized intercepts $\hat{b}$; as a function of Mach number

\begin{tabular}{lllllll}
\hline & $\begin{array}{l}T u_{p 0} \\
(15 \%)^{\#}\end{array}$ & $\begin{array}{l}T u_{p 0} \\
(50 \%)^{\S}\end{array}$ & $b$ & $\begin{array}{l}1 / \hat{T} u_{p 0} \\
(15 \%)^{\#}\end{array}$ & $\begin{array}{l}1 / \hat{T} u_{p 0} \\
(50 \%)^{\S}\end{array}$ & $\hat{b}$ \\
\hline$M=0.35$ & $1.3 \times 10^{-3}$ & $5.6 \times 10^{-4}$ & 3.99 & 1.00 & 1.00 & 1.00 \\
$M=0.50$ & $2.1 \times 10^{-3}$ & $6.9 \times 10^{-4}$ & 3.15 & 0.62 & 0.81 & 0.79 \\
$M=0.65$ & $3.6 \times 10^{-3}$ & $9.5 \times 10^{-4}$ & 1.73 & 0.36 & 0.60 & 0.43
\end{tabular}

${ }^{\#}$ The values in brackets give the percentage of test section width at which the measurement was conducted: $15 \%$ corresponds to a measurement close to the wall. ${ }^{\S} 50 \%$ corresponds to the center of the test section.

with a coefficient of determination of $R^{2}=0.979$. The linear decrement of $\bar{b}$ shows that an increase in Mach number leads to a reduction of the transition Reynolds number. This fact can also be seen in Fig. 4.6 directly, by comparing the transition Reynolds numbers for different Mach numbers at a constant $\beta_{H}$. These results appear to stand in contrast with the stabilizing effect of compressibility predicted by linear stability theory (Arnal and Vermeersch, 2011). However, also the variation of the free-stream disturbance environment as a function of the Mach number has to be taken into account. As described in Sect. 4.3 , the total pressure turbulence level, $T u_{p 0}$, increases with $M$ in the investigated Mach number range, which may outweigh the stabilizing effect of compressibility.

The value of $T u_{p 0}$ was linearly extrapolated to $M=0.35$ from Fig. 4.35 of Koch (2004). The values of $T u_{p 0}$ at $M=0.50$ and 0.65 were then normalized by the extrapolated value at $M=0.35$, with $\hat{T u_{p 0}}=T u_{p 0} / T u_{p 0}(M=0.35)$. Since an inverse relation to the transition Reynolds number is expected (Schlichting and Gersten, 2000) the values of $\hat{T} u_{p 0}$ were additionally inverted (Table 4.2). Also the intercepts, $b$, were normalized by their value at $M=0.35: \hat{b}=b / b(M=0.35)$.

The normalized (and inverted) values are summarized in Table 4.2 for comparison. It can be seen that the values of $\hat{b}$ are bounded by the values of $1 / \hat{T} u_{p 0}$ with the lower bound given by $1 / \hat{T} u_{p 0}$ at $15 \%$ test section width and the upper bound given by $1 / \hat{T} u_{p 0}$ at $50 \%$ test section width. It shows that the change in transition Reynolds number (represented by $\hat{b}$ ) is in inverse proportion to the change in turbulence level $\left(\hat{T} u_{p 0}\right)$ with Mach number: $\hat{b} \sim 1 / \hat{T} u_{p 0}$. This result suggests that the increase in total pressure turbulence level is very likely the cause for the observed decrease in transition Reynolds number with Mach number.

\subsubsection{Compressible and incompressible $N$-factor analysis}

The critical $N$-factors were determined with compressible (Fig. 4.7) and incompressible theory (Fig. 4.8). In the compressible case the determined critical $N$-factors vary between 6.7 and 10.2 , while in the incompressible case the variation with $\beta_{H}$ is smaller and the determined critical $N$-factors lay between 7.7 and 10.1 .

The average critical $N$-factor in DNW-KRG for the modified PaLASTra model at zero pressure gradient $\left(H_{12, \text { inc }}=2.59\right)$ is about 9.0 for the compressible $N$-factor calculation and at about 9.5 


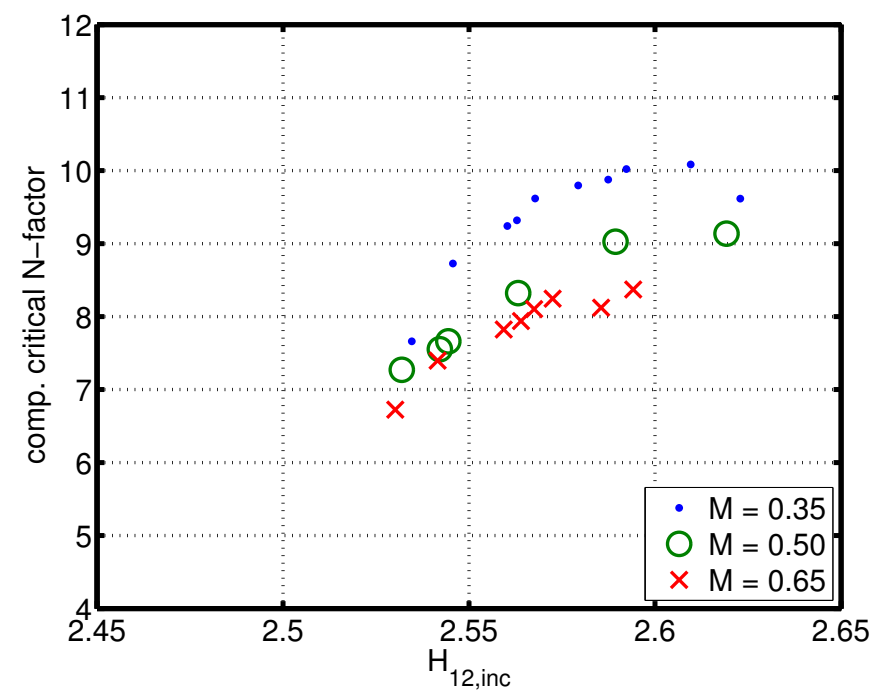

Figure 4.7: Compressible critical $N$-factor as a function of $H_{12, \text { inc }}$ for a Mach number range of $M=0.35-0.65$ and a chord Reynolds number of $R e_{c}=6 \cdot 10^{6}$

in the case of incompressibility, with a variation with $M$ of $\Delta N_{\text {crit,comp }} \approx \pm 1$ and $\Delta N_{\text {crit, inc }} \approx \pm 0.5$ respectively.

It can be seen that in the compressible and incompressible case the determined critical $N$-factors show a dependency on $H_{12, \text { inc }}$. This dependence was observed before and can be accounted to shortcomings of the $e^{N}$-method (Arnal et al., 1997).

In the compressible case the determined critical $N$-factor decreases with increasing Mach number, as it would be expected by Mack's relation based on the turbulence level of velocity fluctuations $T u_{u}=\sqrt{\frac{1}{3}\left(u^{\prime 2}+v^{\prime 2}+w^{\prime 2}\right)} / U_{\infty}$ for $T u_{u} \geq 10^{-3}$ Mack, 1977):

$$
N_{T}=-8.43-2.4 \ln \left(T u_{u}\right)
$$

In contrast to the compressible case, the incompressible case does not show a clear trend with Mach number, as it would be expected by Mack's relation (Eq. 4.4).3

\subsection{Conclusions}

The effect of Mach number and pressure gradient on the transition Reynolds number was investigated in DNW-KRG. The expected stabilizing effect of compressibility on boundary layer transition, as predicted by linear stability theory, could not be observed. This behavior is likely due to an increase of the total pressure turbulence level with Mach number in DNW-KRG.

The modification of the PaLASTra model proved to be useful in order to reduce the magnitude of pressure fluctuations, caused by flow separation at the trailing edge of the model, below the minimum measurable quantity. A linear relation between Hartree parameter and transition Reynolds

\footnotetext{
${ }^{3} \mathrm{~A}$ direct comparison with the critical $N$-factor based on Mack's relation is not possible, as the velocity turbulence level, $T u_{u}$, of DNW-KRG is unknown, due to measurement difficulties of the velocity turbulence level in DNW-KRG (Koch, 2004).
} 


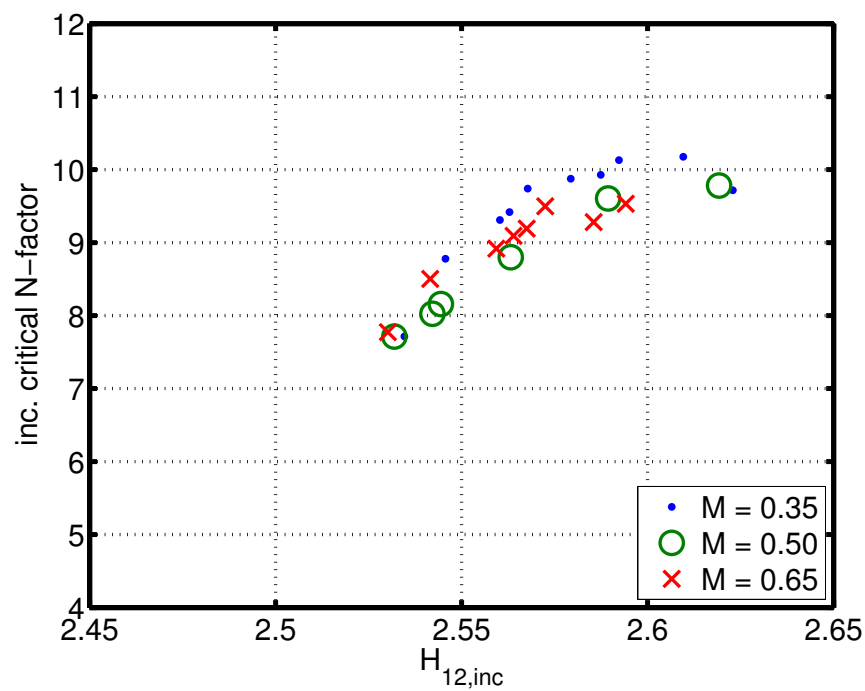

Figure 4.8: Incompressible critical $N$-factor as a function of $H_{12, i n c}$ for a Mach number range of $M=0.35-0.65$ and a chord Reynolds number of $R_{c}=6 \cdot 10^{6}$

number was found. It can be concluded that the modified PaLASTra model can be a useful tool to study laminar-turbulent transition in two-dimensional flows.

A critical $N$-factor of about 9.0, for compressible calculations, and 9.5 for incompressible calculations, was found for PaLASTra in DNW-KRG. This result proves that even though the total pressure turbulence level of DNW-KRG increases with Mach number, the wind tunnel is well suited for laminar flow investigations.

\section{Acknowledgements}

We would like to thank L. Schojda, L. Koop and H. Rosemann for their support and advise; V. Ondrus (Univ. Hohenheim), T. Kleindienst (DLR), C. Fuchs (DLR) for model and test preparation; J. Ost, M. Hilfer and U.Henne for their help during the measurement campaign, data evaluation and interpretation (all DLR). Furthermore we would like to thank K. Plettenberg, R. Kahle, M. Aschoff, and S. Hucke (German-Dutch Wind Tunnels) for the support during the wind-tunnel test campaigns. 



\section{Unit Reynolds number, Mach number and pressure gradient effects on laminar-turbulent transition in two-dimensional boundary layers (Risius et al., 2018b)}

Citation and credit: Reprinted from Experiments in Fluids, volume 59, article 86, 2018, Copyright 2018, The Authors doi:10.1007/s00348-018-2538-8

Reference: Risius et al. (2018b)

Title: "Unit Reynolds number, Mach number and pressure gradient effects on laminar-turbulent transition in two-dimensional boundary layers"

Authors: Steffen Risius, Marco Costantini, Stefan Koch, Stefan Hein and Christian Klein

\section{Contributions of the first author:}

- conducted a literature review on Mach and unit Reynolds number effects and on the influence of free stream turbulence spectra on laminar-turbulent transition

- implemented and carried out the evaluation of the transition locations over the complete span of the PaLASTra model

- investigated the self-similarity of the boundary-layer velocity distribution in connection with the shape factor and Hartree parameter

- analyzed the spectral level of total pressure fluctuations and showed its frequency-dependent influence on the transition Reynolds number

- isolated and quantified the Mach number and unit Reynolds number effects in the Cryogenic Ludwieg-Tube

- implemented corrections of the critical $N$-factor by integrating the influence of the spectral level of total pressure fluctuations on the initial amplitude of Tollmien-Schlichting waves

- developed a correction method of the incidence angle of acoustic disturbances on the critical $N$-factor

- compared critical $N$-factors obtained by compressible and incompressible linear stability analysis with the described correction methods

- quantified the measurement uncertainty and repeatability of the results 
- discussed the results, compared them with literature and drew the conclusions

- made the first and final version of all figures

- wrote the first and final version of the manuscript

- carried out the correspondence with the editor and implemented the corrections suggested by the referees

\subsection{Abstract}

The influence of unit Reynolds number $\left(R e_{1}=17.5 \times 10^{6} \mathrm{~m}^{-1}\right.$ to $\left.80 \times 10^{6} \mathrm{~m}^{-1}\right)$, Mach number $(M=0.35$ to 0.77$)$ and incompressible shape factor $\left(H_{12}=2.50\right.$ to 2.66$)$ on laminar-turbulent boundary layer transition was systematically investigated in the Cryogenic Ludwieg-Tube Göttingen (DNW-KRG). For this investigation the existing two-dimensional wind tunnel model, PaLASTra, which offers a quasi-uniform streamwise pressure gradient, was modified to reduce the size of the flow separation region at its trailing edge. The streamwise temperature distribution and the location of laminar-turbulent transition were measured by means of temperature-sensitive paint (TSP) with a higher accuracy than attained in earlier measurements.

It was found that for the modified PaLASTra model the transition Reynolds number $\left(R e_{t r}\right)$ exhibits a linear dependence on the pressure gradient, characterized by $H_{12}$. Due to this linear relation it was possible to quantify the so-called 'unit Reynolds number effect', which is an increase of $R e_{t r}$ with $R e_{1}$. By a systematic variation of $M, R e_{1}$ and $H_{12}$ in combination with a spectral analysis of freestream disturbances, a stabilizing effect of compressibility on boundary layer transition, as predicted by linear stability theory, was detected ('Mach number effect'). Furthermore, two expressions were derived which can be used to calculate the transition Reynolds number as a function of the amplitude of total pressure fluctuations, $R e_{1}$ and $H_{12}$.

To determine critical $N$-factors, the measured transition locations were correlated with amplification rates, calculated by incompressible and compressible linear stability theory. By taking into account the spectral level of total pressure fluctuations at the frequency of the most amplified Tollmien-Schlichting wave at transition location, the scatter in the determined critical $N$-factors was reduced. Furthermore, the receptivity coefficients dependence on incidence angle of acoustic waves was used to correct the determined critical $N$-factors. Thereby, a found dependency of the determined critical $\mathrm{N}$-factors on $\mathrm{H}_{12}$ decreased, leading to an average critical $\mathrm{N}$-factor of about 9.5 with a standard deviation of $\sigma \approx 0.8$.

\subsection{Introduction}

Natural laminar flow (NLF) technology is a functional technology to reduce wall shear stress and fuel consumption of commercial aircraft (Crouch, 2015; Fujino et al., 2003). It is implemented 
on aerodynamic surfaces with zero to moderate sweep angles, where the predominant instabilities leading to transition to turbulence are Tollmien-Schlichting (T-S) waves. In particular, T-S waves are the main instability mechanism for two-dimensional laminar boundary layers developing on smooth surfaces. They are excited via the receptivity process and the initial stage of their amplification can be described by linear stability theory; in a second amplification stage, the T-S waves become unstable to three-dimensional perturbations, described by secondary instability theory. The secondary instabilities lead to the formation of $\Lambda$-structures which cause 'hair-pin' vortices close to the wall and result in the break down to turbulence (Schlichting and Gersten, 2000). This transition process is also referred to as 'natural' laminar-turbulent transition which is typical for low freestream disturbance environments, as encountered in flight (Joslin, 1998).

However, many conventional wind tunnels have disturbance levels higher than in flight, which can cause the mechanism of 'natural' transition to be changed or bypassed and can lead to discrepancies between transition locations measured in wind tunnels and in flight (Fisher and Dougherty, 1982; Michel and Froebel, 1988). Results from Meier et al. (1987) indicate that the movement of transition location is not determined by the RMS (root mean square) turbulence level, but instead by the spectral level in the frequency range, in which the T-S waves are most unstable. Therefore they concluded from their experiments that "the Reynolds number at which the transition onset was detected can be correlated perfectly with the spectral level in the frequency range representative for the Tollmien-Schlichting waves" (Meier et al., 1987).

\subsubsection{Unit Reynolds number effect}

The described correlation can be used to explain the so-called 'unit Reynolds number effect', which refers to an increase in transition Reynolds number with unit Reynolds number, measured in self-similar boundary layers 11 A concise explanation of the unit Reynolds number effect was given by Arnal and Délery (2004) with the words "when the unit Reynolds number increases (for a fixed value of the Mach number), the range of unstable waves shifts to higher frequencies. In the freestream, the energy of the corresponding pressure fluctuations decreases, so that the transition Reynolds number increases."

\subsubsection{Mach number effect}

By the systematic variation of $M, R e_{1}$ and $H_{12}$ in combination with a spectral analysis of freestream disturbances, it was also possible to investigate the so-called 'Mach number effect' on laminarturbulent transition in two-dimensional boundary layers. The Mach number effect refers to a stabilizing effect of compressibility on boundary layer transition, as predicted by linear stability theory: the consideration of compressibility effects in linear stability theory leads to reduced growth rates of Tollmien-Schlichting waves (Arnal and Vermeersch, 2011). Under the assumption of a constant critical $N$-factor (see below) the reduced growth rates will lead to a delay of laminar turbulent transition (Arnal and Vermeersch, 2011). However, in wind tunnel experiments, the

\footnotetext{
${ }^{1}$ The transition Reynolds number, $R e_{t r}$, is defined as $R e_{t r}=U_{\infty} \cdot x_{t r} / v_{\infty}$, with the transition location, $x_{t r}$, freestream velocity, $U_{\infty}$, and kinematic viscosity, $v_{\infty}$. The unit Reynolds number is defined as $R e_{1}=U_{\infty} / \nu_{\infty}$ in units of $\mathrm{m}^{-1}$.
} 
stabilizing compressibility effect is opposed by an increased initial amplitude of the T-S waves, due to an increased freestream disturbance level with Mach number (Arnal, 1989). Therefore the direct comparison of transition Reynolds numbers, measured at different Mach numbers, is not meaningful, due to changes in the disturbance spectrum, as shown in Sect.5.4.1

\subsubsection{Transition prediction by the $e^{N}$-method and correction of determined critical $N$-factors}

The $e^{N}$-method is based on linear stability theory (Schlichting and Gersten, 2000) and was first published in 1956 (Smith and Gamberoni, 1956; van Ingen, 1956). Since then, it has been widely used for transition prediction on aircraft surfaces (Hue et al., 2015, 2018, Schrauf, 1994, 2000, 2005; Schrauf et al., 1996, 1998; Streit et al., 2011; van Ingen, 2008; Voogt, 1996). The $N$-factor method uses growth rates of T-S waves in a laminar boundary layer calculated by linear stability theory and assumes that transition takes place where the most unstable disturbances are amplified by a factor of $e^{N}$, where $N$ is assumed to be a universal constant. However, it has been found that the critical $N$-factor is not a universal constant, but instead depends strongly on the flow conditions (van Ingen, 2008). Therefore it varies for each wind tunnel and model and has to be determined by a semi-empirical method, based on a correlation of stability calculations with experimental data (van Ingen, 2008).

It has been concluded, that the major drawback of the $e^{N}$-method, is its implicit assumption that all unstable waves have the same initial amplitude, which is not possible in practical applications due to variations in the external disturbance spectrum and receptivity (Arnal, 1989). This observation was succinctly worded by Stetson et al. (1986) who stated that "knowledge of the stability characteristics of a boundary layer is only part of the problem. The external disturbances must be prescribed in order to make boundary layer transition prediction based upon stability considerations."

To improve the $N$-factor method Mack (1977) made one of the earliest attempts to take the turbulence level of velocity fluctuations, $T u_{u}=\sqrt{\frac{1}{3}\left(u^{\prime 2}+v^{\prime 2}+w^{\prime 2}\right)} / U_{\infty}$, into account and corrected the critical $N$-factor with

$$
N=-8.43-2.4 \ln \left(T u_{u}\right)
$$

For a turbulence intensity of $0.07 \%$ (which is typical of low turbulence subsonic wind tunnels (Michel and Froebel, 1988) Mack's correlation gives a critical $N$-factor of $N_{c r i t}=9$. However, it has been found that Mack's method is most useful at high turbulence levels above $T u_{u} \approx 0.1 \%$, while it shows much scatter in low-turbulence environments, as relevant for this study (van Ingen, 2008).

The low reliability in low-turbulence environments may be explained by the fact that the RMS turbulence level is not sufficient to describe the disturbance environment. Instead, the spectral level in the frequency range representative for Tollmien-Schlichting waves leading to transition has to be considered as described above.

Besides total pressure fluctuations also static pressure fluctuations have to be considered. However, the static pressure fluctuations in DNW-KRG remain almost constant for the investigated frequency, Mach number and Reynolds number ranges (Koch, 2004). 


\subsubsection{Compressible and incompressible $N$-factor analysis}

The linear stability analysis, used for the $N$-factor method, can be carried out either with or without incorporation of compressibility effects (Schrauf, 2006). It has been found that critical $N$-factors exhibit a larger scatter when they are calculated with compressible linear stability theory, than in the case of incompressible linear stability theory (Schrauf, 1994, 2000, Schrauf et al., 1998). This observation contradicts the general expectation that a model which incorporates more physical processes (i.e. compressibility effects) produces more consistent results. Therefore it has been conjectured that compressibility effects in linear stability theory may be compensated by another physical mechanism that leads to less consistent results of the determined compressible critical $N$-factors (Schrauf, 2000).

\subsubsection{Scope of the work}

\subsubsection{Unit Reynolds number effect}

The unit Reynolds number effect has been observed in various hypersonic facilities (McCauley et al. 1966: Softley et al., 1969; Stainback, 1967; Stainback et al., 1974) and was carefully investigated by Stetson et al. (1986). However, it has never been investigated in a large subsonic flow regime before, which is most relevant for commercial aircraft today. To examine the unit Reynolds number effect for the transonic Cryogenic Ludwieg-Tube Göttingen (DNW-KRG), a systematic experimental investigation of the influences of unit Reynolds number $\left(R e_{1}=17.5 \times 10^{6} \mathrm{~m}^{-1}\right.$ to $\left.80 \times 10^{6} \mathrm{~m}^{-1}\right)$, incompressible shape factor $\left(H_{12}=2.50\right.$ to 2.66$)$ and Mach number $(M=0.35$, 0.50 and 0.65 ) on transition Reynolds number was conducted in this study. In DNW-KRG unit Reynolds numbers of up to $R e_{1}=400 \times 10^{6} \mathrm{~m}^{-1}$ can be achieved for two-dimensional test models by decreasing temperature down to $100 \mathrm{~K}$ and increasing pressure up to 10 bar (Rosemann, 1997). In the current study the charge temperature was kept constant at about $283 \mathrm{~K}$, while the charge pressure of the wind tunnel was varied to adjust the unit Reynolds number 2 The Mach number of the flow was varied by adjusting the cross section of the sonic throat downstream of the test section (Koch, 2004), while a variation of the shape factor was achieved by varying the angle-of-attack of the model (Costantini, 2016, Risius et al. 2018a). Limitations to the test envelope were due to: (a) the maximal charge pressure of DNW-KRG, which limited the maximal unit Reynolds number, (b) boundary-layer separation downstream of a pressure minimum in the leading-edge area at high angles-of-attack, (c) blockage effects of the model inside the test section at large negative angles-of-attack, which limited the range of examinable pressure gradients (shape factors), and (d) the chord length of the model, which limited the detectable transition location.

Due to the systematic approach, the current work exceeds earlier investigations on the unit Reynolds number effect, as it does not only give a qualitative explanation, but allows a quantitative calculation of the transition Reynolds number as a function of incompressible shape factor and unit Reynolds number for three different Mach numbers.

\footnotetext{
${ }^{2}$ The 'charge temperature' and 'charge pressure' are the working gas temperature and pressure at rest before the fast-acting valve of DNW-KRG is opened and the test run is initiated.
} 


\subsubsection{Mach number effect}

Due to the inherent coupling of Mach number and freestream disturbance spectrum the direct comparison of transition Reynolds numbers, measured at different Mach numbers, is not meaningful. However, in this paper it will be shown that a comparison of transition Reynolds numbers, obtained at different Mach numbers, can be conclusive if the total pressure disturbance spectrum is approximated as a function of $\operatorname{Re}_{1}, H_{12}$ and $M$ and if this approximation is used to express the transition Reynolds number as a function of the incompressible shape factor and of the spectral level of total pressure fluctuations.

\subsubsection{Correction of compressible and incompressible critical $N$-factors}

Incompressible and compressible stability theory was employed to calculate critical $N$-factors for all available Mach and Reynolds numbers. To conduct a correction of the determined critical $\mathrm{N}$-factors, the spectral level of total pressure fluctuations in the frequency range of T-S waves leading to transition was used. It will be shown that this correction leads to a significantly better correlation of the determined compressible critical $N$-factors.

To correct the influence of acoustic disturbances in the present study, the dependence found by Heinrich et al. (1988) was approximated linearly and used to correct the influence on the determined critical $N$-factors of the incidence angle of acoustic waves reaching the boundary layer. It was assumed that this incidence angle of the acoustic waves is equal to the angle-of-attack of the model in the wind tunnel.

\subsubsection{Outline of the paper}

In the next section of the paper (Sect. 5.3), the experimental setup, TSP data analysis, boundary layer computations and linear stability calculations are described.

In Sect. 5.4 first a spectral analysis of total pressure fluctuations, measured in the freestream of the wind tunnel, is presented (Sect. 5.4.1). Afterwards a method to correct the influence of non-adiabatic surface temperatures on the transition location, is shown (Sect. 5.4.2). Then, the influence of the incompressible shape factor on transition Reynolds number is approximated linearly for all Mach and unit Reynolds numbers (Sect. 5.4.3). These relations are used to calculate the transition Reynolds number as a function of the unit Reynolds number with the help of a power law approximation (Sect. 5.4.4). Afterwards, a function is derived which gives the most amplified frequency of T-S waves at transition location depending on unit Reynolds number and incompressible shape factor (Sect. 5.4.5). In the last part of Sect. 5.4, the above relations are combined to give the transition Reynolds number as a function of the spectral level of total pressure fluctuations for both approximations derived (Sect. 5.4.6). These relations allow a comparison of the transition Reynolds numbers measured at different Mach numbers and, therefore, different total pressure turbulence levels.

In Sect. 5.5, the determined critical $N$-factors of incompressible and compressible linear stability theory are presented. The correction of compressible critical $N$-factors is carried out by correcting initial amplitudes with the spectral level of total pressure fluctuations in the frequency range relevant 
for T-S induced transition (Sect. 5.5.1). Furthermore, the receptivity coefficients dependence on incidence angle is used to correct determined critical $N$-factors (Sect. 5.5.2).

Measurement uncertainties and repeatability of the results are analyzed and discussed in Sect.5.6. In the last parts of the paper the main outcome of the analysis is summarized and discussed (Sect.5.7) and finally a conclusion is drawn (Sect. 5.8).

\subsection{Experimental setup and boundary layer computations}

The experimental data presented in this paper originates from six different measurement campaigns of the modified PaLASTra wind tunnel model that were carried out over a time span of two years. Most of the data presented was taken during the first wind tunnel entry, while later measurements, marked as 'later entry' (abbreviation: 'l.e.'), were used only for completion of the data and check of repeatability.

\subsubsection{The Cryogenic Ludwieg-Tube Göttingen}

The experiments were performed in the transonic Cryogenic Ludwieg-Tube Göttingen (Koch, 2004; Rosemann, 1997). The blow down wind tunnel is operated intermittently with gaseous nitrogen as driving gas and has good flow quality. The total temperature turbulence leve ${ }^{3}, T u_{T_{0}}$, in the center of the test section, is lower than $0.04 \%$ at Mach number $M=0.8$, at unit Reynolds number of $R e_{1}=30 \times 10^{6} \mathrm{~m}^{-1}$ and a charge temperature, $T_{c} \approx 282 \mathrm{~K}$, and it decreases with lower Mach numbers. The mass flux turbulence leve ${ }^{3}, T u_{\rho u}$, in the center of the test section is approximately $0.06 \%$ at $M=0.8, T_{c} \approx 283 \mathrm{~K}$ and $30 \times 10^{6}<R e_{1}<77 \times 10^{6} \mathrm{~m}^{-1}$; it increases slightly at lower Mach numbers, but remains smaller than $0.08 \%$ (Koch, 2004). To guarantee an interference-free flow around the wind tunnel model, the upper and lower test section walls were adapted (Rosemann, 1997). The uncertainties in the inflow Mach and Reynolds numbers in the present work were within $M= \pm 0.002$ and $R e_{1}= \pm 0.25 \times 10^{6} \mathrm{~m}^{-1}$.

\subsubsection{The two-dimensional wind tunnel model PaLASTra}

The wind tunnel model used for the present study was the two-dimensional PaLASTra model (Costantini, 2016; Costantini et al. 2015b, 2016ab). On the lower surface of the original PaLASTra model an abrupt contour change was imposed at $x / c=80 \%$ (Fig. 5.1), to fix boundary layer separation (Costantini et al., 2016a). However, the large separation region, originating downstream of the original PaLASTra model, leads to strong pressure fluctuations (i.e. acoustic noise) inside the test section; these are likely to increase the initial amplitude of the disturbances within the laminar boundary layer (through the receptivity process) and thus reduce transition Reynolds numbers (Costantini et al., 2016a).

To reduce the separation region, an additional aft part was designed and attached to the original PaLASTra model as shown in Fig. 5.1. This modification of the model reduces the size of the

\footnotetext{
${ }^{3}$ The turbulence level of a quantity $x$ is defined as $T u_{x}=\sqrt{\overline{(x-\bar{x})^{2}}} / \bar{x}=x_{R M S} / \bar{x}$, where $\bar{x}$ is the temporal average of $x$ and $x_{R M S}$ is the RMS of the fluctuations.
} 


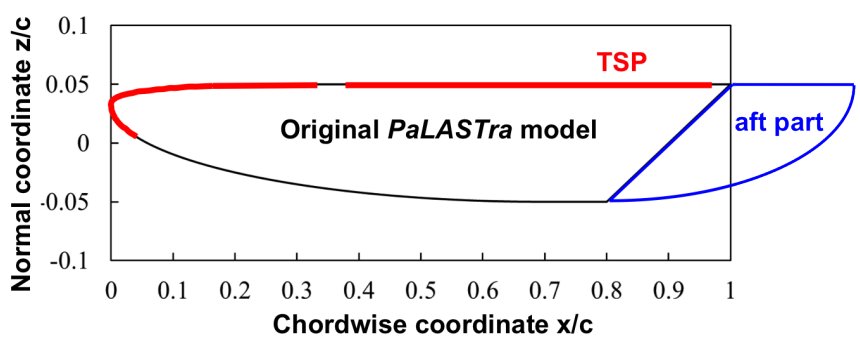

Figure 5.1: Side view of the original PaLASTra model with an additional aft part at the trailing edge and Temperature-Sensitive Paint (TSP) on top. The original chord length of $c=0.2 \mathrm{~m}$ is used for normalization of the chordwise coordinate

separation region and the magnitude of the emitted pressure fluctuations. After modification, the separation-induced pressure fluctuations were below the minimum observable quantity of a Kulite pressure transducer (CCQ-093), which was operated at up to $200 \mathrm{kHz}$ and mounted flush with the test section side wall at a location upstream of the model 4

A typical pressure distribution on the upper side of the modified PaLASTra model, measured by pressure taps (with $0.25 \mathrm{~mm}$ diameter), is shown in Fig. 5.2. Downstream of the leading edge region with $x / c>20 \%$, the pressure gradient is essentially uniform on a large portion of the upper surface. Only around $x / c=35 \%$ the pressure distribution shows some slight variations from an ideally smooth one, due to a model part junction (Costantini, 2016; Costantini et al., 2015b, 2016a).

Because T-S waves and the transition position are very sensitive to the surface quality great care was taken to achieve smooth surface conditions. The surface waviness was less than $h / a \leq 0.0025$, where $h$ is half the amplitude of the wave and $a$ corresponds to its quarter wavelength in streamwise direction, thus fulfilling the criteria for allowable waviness for laminar flow (Carmichael, 1959; Fage. 1943; Gluyas, 1967). The model surface was polished to an average roughness of $R_{a}=0.038 \mu \mathrm{m}$ with a standard deviation of $0.01 \mu \mathrm{m}$, while the mean roughness depth was $R_{z}=0.32 \mu \mathrm{m}$ with a standard deviation of $0.11 \mu \mathrm{m}$. In the leading edge region the average roughness and mean roughness depth were even further reduced to $R_{a}=0.027 \mu \mathrm{m}$ and $R_{z}=0.20 \mu \mathrm{m}$. The step at the model part junction at $x / c=35 \%$ was less than $0.5 \mu \mathrm{m}$ (Costantini, 2016).

Non-intrusive global measurement of the surface temperature distribution was carried out using a temperature-sensitive paint (Ondrus et al. 2015). TSP formulation, surface quality, optical setup, acquisition and evaluation of the TSP images were the similar to the ones described in (Costantini et al. 2016a). Enhancements were made in the data acquisition by installation of new LEDs to illuminate the TSP, leading to an increased temporal resolution and contrast of TSP result images. Furthermore, a new camera setup was developed; it was used during the later entries of the PaLASTra model and increased the spatial resolution, temporal resolution and contrast of the result images even further.

The transition detection in the current study was conducted by the maximum gradient technique, which has been described by Costantini (2016) and Costantini et al. (2016a). However, it was not

\footnotetext{
${ }^{4}$ The minimum observable quantity is restricted by the amplitude of pressure fluctuations emitted by the turbulent boundary layer of the test section side wall in which the Kulite pressure transducer was mounted.
} 


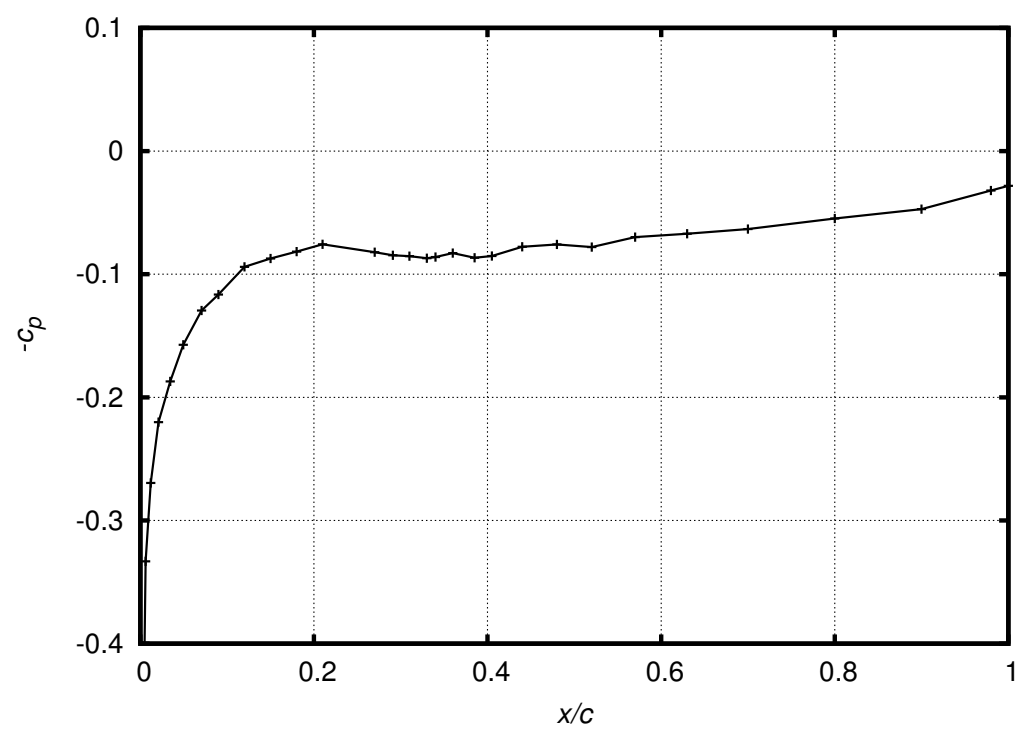

Figure 5.2: Chordwise evolution of pressure coefficient, $c_{p}$, on model upper side at $\operatorname{Re}_{1}=$ $30 \times 10^{6} \mathrm{~m}^{-1}, M=0.65$ and an angle-of-attack of $-1.5^{\circ}$. The measurement uncertainty of the pressure tap measurement leads to an error of $c_{p} \lesssim \pm 0.005$ (not shown in the plot). The flow conditions are the same as in Fig. 5.3 - Fig. 5.8

only conducted at ten spanwise locations, as in earlier studies (Costantini, 2016, Costantini et al., 2015b, 2016a), but in this work it was extended to almost the complete span. Side wall effects, turbulent wedges and significant flow disturbances were excluded from the transition detection, as shown in Fig. 5.3. The RMS of the variation in transition location along the span was determined for each data point and plotted as an error bar in Fig. 5.10 .

To determine the surface temperature distribution on the upper side of the model, the TSP was calibrated in an external calibration chamber (Egami et al., 2012). Surface temperature distributions in the streamwise direction, which were extracted from the TSP data at five spanwise sections, are shown in Fig. 5.4.

The described improvements in the measurement technique allowed the determination of transition locations with a higher accuracy than in earlier measurements (Costantini, 2016, Costantini et al. 2012, 2015b, 2016a b) as well as the correction of temperature effects (see Sect. 5.4.2). These advances made it possible to conduct a systematic analysis of unit Reynolds number, Mach number and pressure gradient effects on transition, as described in Sect. 5.4

\subsubsection{Boundary layer computations}

Laminar boundary layer computations were performed using the compressible boundary layer solver COCO (Schrauf, 1998), which was modified in order to incorporate not only the measured surface pressure but also the measured surface temperature distributions as inputs. Thus, it was accounted for the influence of the non-adiabatic surface temperature on the boundary layer. COCO calculates a fully laminar boundary layer, which was used to determine incompressible displacement 


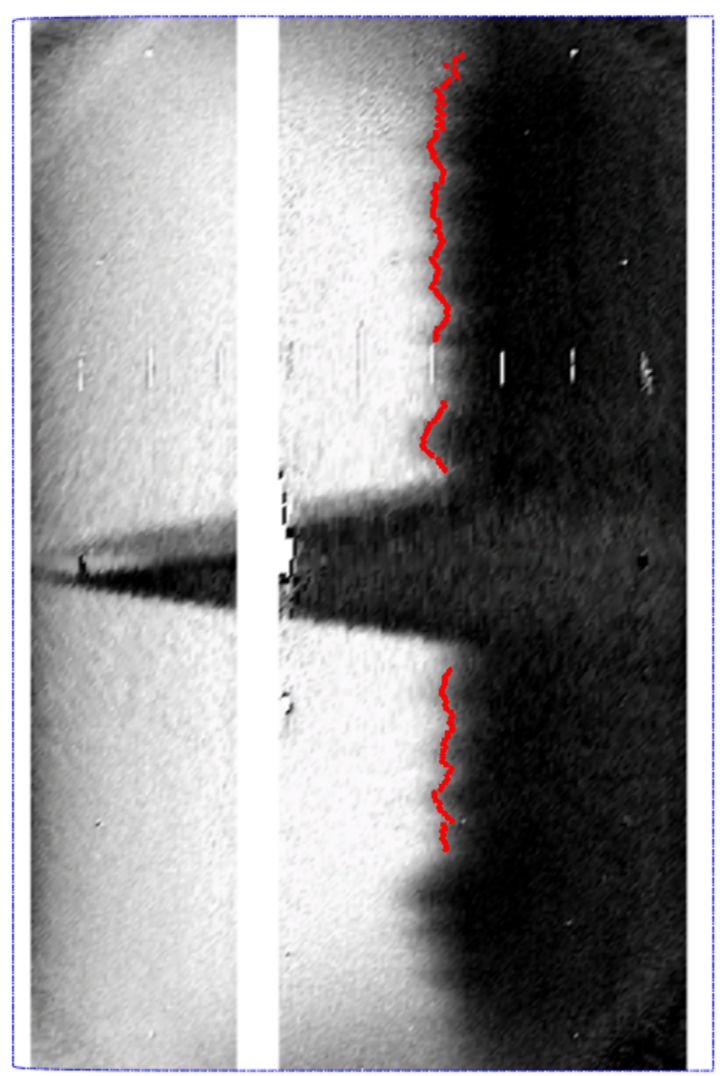

Figure 5.3: TSP result image where bright and dark areas correspond to laminar and turbulent regions, respectively. The flow direction is from left to right. The whitened strips indicate metallic surfaces of the model where no TSP had been applied. Markers indicating every $10 \%$ chord are visualized by thin white lines. The two turbulent wedges in the mid-span domain are caused by pressure taps in the leading edge region. The red line indicates the detected transition location by the maximal gradient technique at the undisturbed flow locations (Costantini, 2016, Costantini et al., 2016a). The flow conditions are the same as in Fig. 5.2

$\left(\delta_{1}\right)$ and momentum thickness $\left(\delta_{2}\right)$ of the laminar boundary layer. The average incompressible shape factor ${ }^{5}, H_{12}=\delta_{1} / \delta_{2}$, was determined by averaging the incompressible shape factor curve between $24 \%<x / c<90 \%$ to characterize the boundary layer velocity profile (Fig. 5.5) 6 . As an alternative to the use of $H_{12}$ to quantify the influence of the pressure gradient, it is also possible to use the Hartree parameter $\beta_{H}$, based on the average pressure gradient, $\partial c_{p} / \partial x$, over the region $24 \%<x / c<90 \%$ (see Fig. 5.5) with (Meyer and Kleiser, 1989):

$$
\beta_{H}=\left(\frac{1}{2}-\frac{1-c_{p}}{x\left(\partial c_{p} / \partial x\right)}\right)^{-1}
$$

\footnotetext{
${ }^{5}$ The incompressible shape factor was used instead of the compressible shape factor, to allow a comparison of different Mach numbers at the same pressure gradient.

${ }^{6}$ The same analysis was carried out for an averaged shape factor curve between $60 \%<x / c<90 \%$, to exclude discontinuities of the pressure distribution, caused by the model part junction at $x / c=35 \%$. The obtained results show the same trends as the ones presented here, but with a smaller standard deviations in the shape factor.
} 

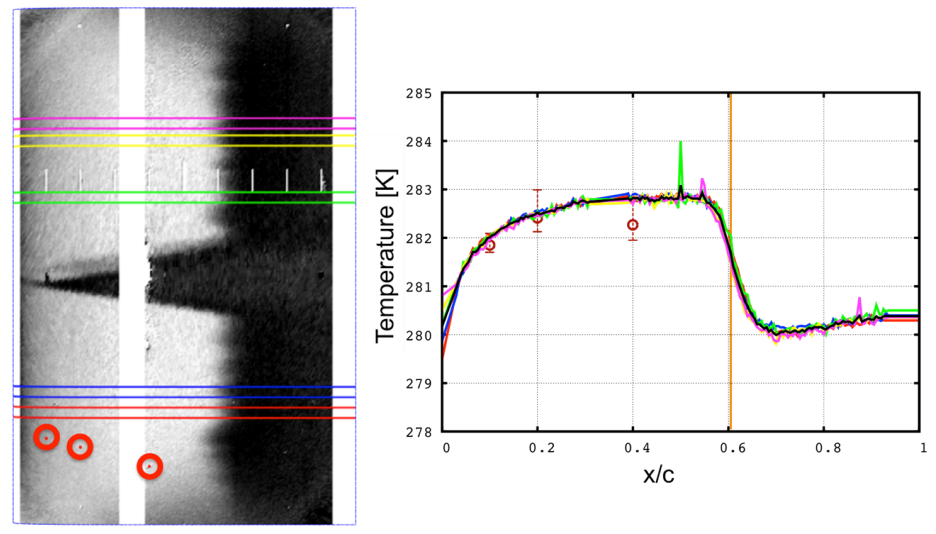

Figure 5.4: Left image: The same TSP result image as shown in Fig. 5.3 with temperature evaluation of TSP (Risius et al. 2018a). Red circles indicate the location of thermocouples embedded in the TSP (Costantini et al., 2016a). Right image: Temperature distribution measured by TSP (colored lines, correspond to those in left image) and their average (black line) as a function of chordwise coordinate (Risius et al., 2018a). The transition location is detected at the maximal temperature gradient at $x / c \approx 61 \%$, marked by a vertical orange line. The flow conditions are the same as in Fig. 5.2

Fig. 5.6 shows the relationship between $H_{12}$ and $\beta_{H}$, which can be approximated for all Mach and unit Reynolds numbers by $\beta_{H}=-0.687 \cdot H_{12}+1.810$. The use of $\beta_{H}$ for the quantification of the pressure gradient leads to similar results as the use of $H_{12}$ (Risius et al., 2018a). However, it can be seen from Fig. 5.6 that the intercept with the $y$-axis of the linear approximation depends on the Mach number. Furthermore, it should be noted that $\beta_{H}$ is not constant over the complete upper surface (Costantini et al. 2016a). For these reasons, the use of $H_{12}$ appears more appropriate for the analysis presented in this paper.

The results of boundary layer computations are also plotted as streamwise flow velocity, $u$, normalized by streamwise velocity at the boundary layer edge, $u_{e}$, against the normalized distance from the wall, $y / \delta_{1}$, at different chordwise coordinates, $x / c$ (Fig. 5.7). It can be seen that the upper side of the modified PaLASTra model exhibits a nearly self-similar boundary layer profile downstream of $x / c=5 \%$ (under the assumption of laminarity over the whole model surface). The same trend was observed for other unit Reynolds numbers. The boundary layer flow developing along the PaLASTra model can be regarded as self-similar for the current investigation.

\subsubsection{Linear stability analysis}

The boundary-layer velocity profiles that were calculated with $C O C O$ are used to conduct a local linear stability analysis by solving the Orr-Sommerfeld equation, which is a fourth-order differential equation, used to calculate amplification rates of T-S waves (Orr, 1907; Sommerfeld, 1908). It has been shown that for incompressible two-dimensional flow configurations the two-dimensional perturbations are most unstable (Squire, 1933). This assumption, known as Squire's theorem, is strictly only valid for incompressible flows. However, it has been shown that it is also a valid for 
the flow conditions investigated in this study (Arnal, 1992, Costantini et al., 2015a, 2016a). The ratio between the amplitude $A$ at a streamwise position $x$ and the initial amplitude $A_{0}$ at the initial position $x_{0}$ is given by $A / A_{0}=e^{N}$, where $N$ is determined by the envelope strategy which uses the most amplified T-S wave at the transition location. Amplification rates of T-S waves for the computed boundary layer were determined by means of $L I L O$ (Schrauf, 2006). According to linear, local stability theory and the quasi-parallel flow assumption, compressible and incompressible stability computations were carried out and their results were correlated with the measured transition location to assess critical $\mathrm{N}$-factors (Fig. 5.8). Furthermore, the frequency of the most amplified T-S wave at the transition location, $f_{t r}$, was determined.

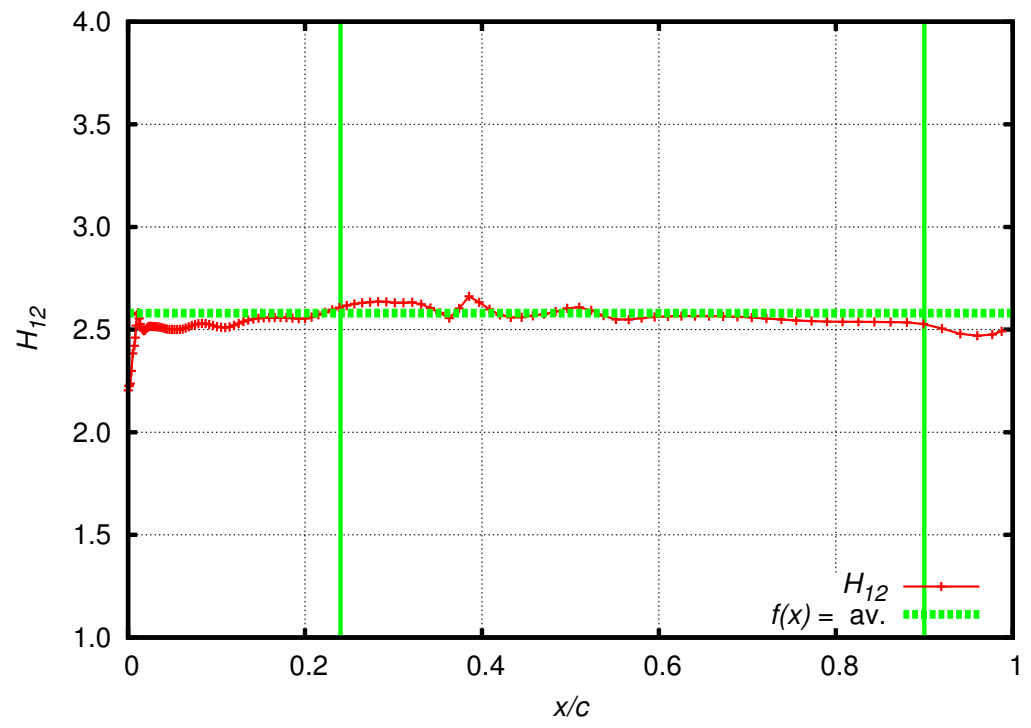

Figure 5.5: Distribution of incompressible shape factor, $H_{12}$, on the model upper side, computed for the same test conditions as in Fig. 5.2 under the assumption of a completely laminar boundary layer. The continuous green lines at $x / c=0.24$ and 0.9 indicate the region where the shape factor was averaged to determine $H_{12}$, which is used for the further analysis. The average value of $H_{12}$ is visualized by the green dotted line. For quantification of the measurement uncertainty, the RMS was also determined (as shown in Figs. 5.10 and 5.15

\subsection{Analysis of stability modifiers}

The influences of pressure gradient and unit Reynolds number on transition Reynolds number were analyzed separately for $M=0.35,0.50$ and 0.65 . Detailed results will be mainly shown for $M=$ 0.35, while data from the other Mach numbers will be summarized in tables and shown in Figs. A.1 to A.4 in the Appendix. For better readability, unit Reynolds numbers and transition Reynolds numbers will be normalized, leading to the definition of $R e_{1}^{*}=R e_{1} /\left(10^{6} \mathrm{~m}\right)$ and $R e_{t r}^{*}=R e_{t r} / 10^{6}$, respectively.

In the following analysis, important equations are labeled with roman numbers, to allow easier 


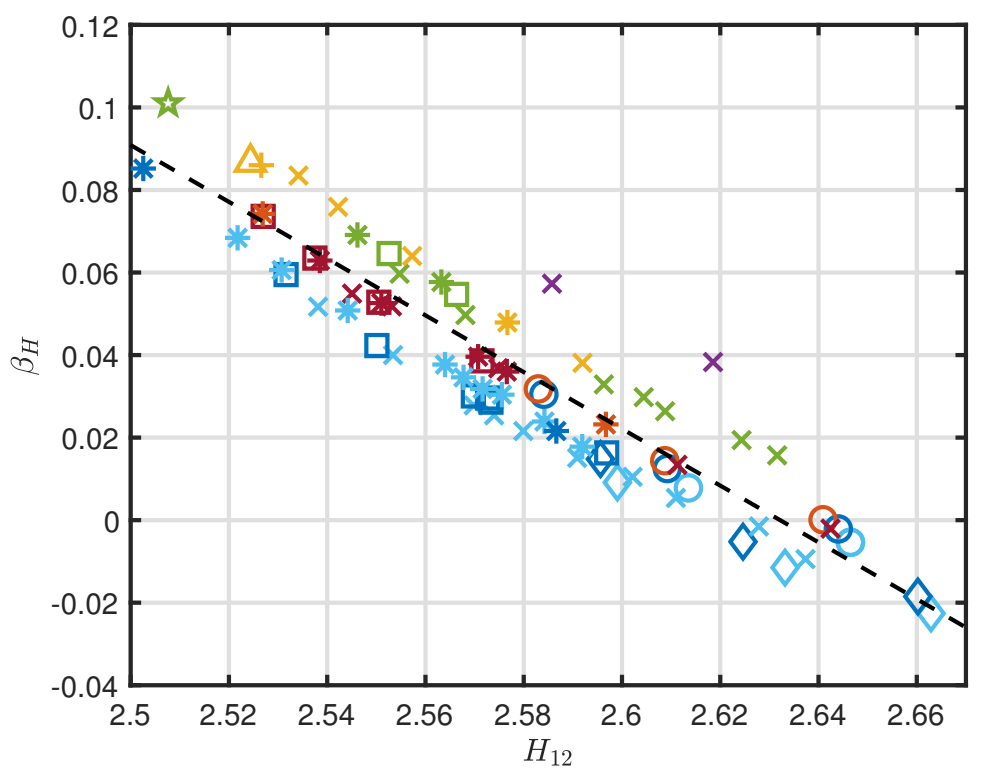

Figure 5.6: Hartree parameter, $\beta_{H}$, as a function of shape factor, $H_{12}$, for all available Mach numbers $(M=0.35$ (blue), 0.50 (red), 0.65 (green/yellow) and 0.77 (purple)) and unit Reynolds numbers $\left(R e_{1}=17.5 \times 10^{6} \mathrm{~m}^{-1}\right.$ to $\left.80 \times 10^{6} \mathrm{~m}^{-1}\right)$. The legend is shown in Fig. 5.22

referencing of the coefficients later on. Slopes are labeled with $\alpha$ and intercepts with $\beta$. Coefficients that are used directly in the approximation of the shape factor are labeled with $h_{i}$, where $i=0,1$, or 2, depending on the degree of $H_{12}^{i}$, to which $h_{i}$ corresponds.

\subsubsection{Spectral analysis of total pressure fluctuations}

To quantify flow disturbances relevant for the amplification of T-S waves leading to transition, the normalized spectral level of total pressure fluctuations, $p^{\star}=p_{0} / / \overline{p_{0}}$, of a measurement conducted by Koch (2004) was reanalyzed. In the log-log plot the frequency dependency of $p^{\star}$ was approximated between $390 \mathrm{~Hz}$ and $10 \mathrm{kHz}$ by the following relation (shown by black dashed lines in Fig. 5.9]:

$$
\log \left(p^{\star}\right)=\alpha_{\mathrm{I}, M} \cdot \log (f)+\beta_{\mathrm{I}, M}
$$

To calculate $\alpha_{\mathrm{I}, M}$ and $\beta_{\mathrm{I}, M}$ at any Mach number, the coefficients $\alpha_{\mathrm{I}, M}$ and $\beta_{\mathrm{I}, M}$, measured at $M=0.45,0.50,0.55,0.60$ and 0.65 (Koch, 2004), were approximated by another set of linear functions, with $\alpha_{\mathrm{I}, M}=0.036 \cdot M-0.458$ and $\beta_{\mathrm{I}, M}=1.537 \cdot M-3.462$, which leads to the following approximation of $p^{\star}: 7$

$$
p^{\star}=f^{\alpha_{\mathrm{I}, M}} \cdot 10^{\beta_{\mathrm{I}, M}}=f^{0.036 \cdot M-0.458} \cdot 10^{1.537 \cdot M-3.462}
$$

The approximated coefficients $\alpha_{\mathrm{I}, M}$ and $\beta_{\mathrm{I}, M}$ are summarized in Table 5.1 and shown by a yellow line for $M=0.35$ in Fig. $5.9{ }^{8}$

\footnotetext{
${ }^{7}$ In this study the approximation is also used for frequencies above $10 \mathrm{kHz}$.

${ }^{8}$ The same analysis can be carried out with a dimensionless frequency $F=2 \pi f v / U_{\infty}^{2}$ that leads to the following approximation of the second coefficient: $\beta_{\mathrm{I}, M}=1.530 \cdot M-7.367$. However, to calculate the transition Reynolds number
} 


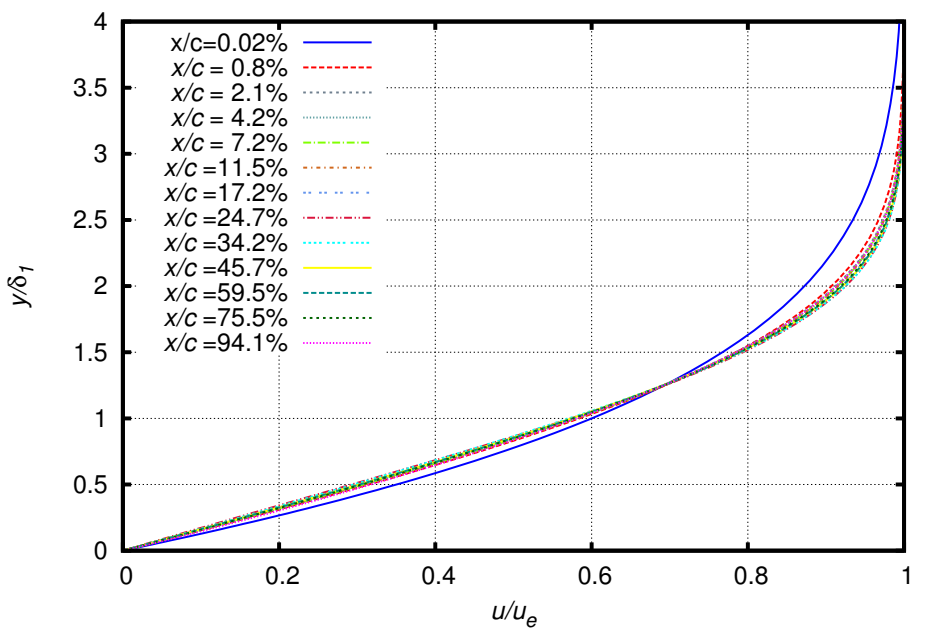

Figure 5.7: Streamwise flow velocity, $u$, normalized by streamwise velocity at the boundary layer edge, $u_{e}$, plotted against distance from the wall, $y$, normalized by incompressible boundary layer displacement thickness, $\delta_{1}$, for different chordwise coordinates, $x / c$. Beyond a chordwise coordinate of about $x / c=5 \%$ the normalized velocity profile remains almost identical. The flow conditions are the same as in Fig. 5.2

It can be seen from Fig. 5.9 that $p^{\star}$ increases with Mach number in the investigated frequency range. Therefore, also the RMS total pressure turbulence level increases with $M$ (Koch, 2004). This fact prohibits a direct comparison of transition Reynolds numbers measured at different Mach numbers (Risius et al., 2018a).

Concerning the unit Reynolds number influences, it has been shown that the total pressure turbulence level (RMS-value) of DNW-KRG increases with unit Reynolds number (Koch, 2004). However, the turbulence level growth is exclusively due to an increasing energy of pressure fluctuations at frequencies below $1.5 \mathrm{kHz}$. When the spectral distribution is analyzed, it can be seen that the energy contained in higher frequencies is independent of the unit Reynolds number and decreases with increasing frequencies (Koch, 2004). The described increase of the total pressure turbulence level (RMS-value) is thus only caused by frequencies below the relevant frequency range of Tollmien-Schlichting waves $\left(5000 \mathrm{~Hz} \lesssim f_{T S} \lesssim 30000 \mathrm{~Hz}\right)$ and has no relevant influence on the current experiment. Therefore, a turbulence level which is independent of unit Reynolds number can be assumed in this analysis.

\subsubsection{Correction of non-adiabatic surface temperature}

Due to the working principle of DNW-KRG, the expanding flow leads to a pressure and temperature drop at the beginning of each test run, which causes a temperature difference between the flow and the surface of the model. Therefore, the (non-adiabatic) model surface temperature, $T_{\text {naw }}$, is generally higher than the adiabatic wall temperature, $T_{a w}$, which enhances boundary layer as a function of the spectral level of total pressure fluctuations a dimensional formulation of the relationship is required (see Secs. 5.4.5 and 5.4.6. 


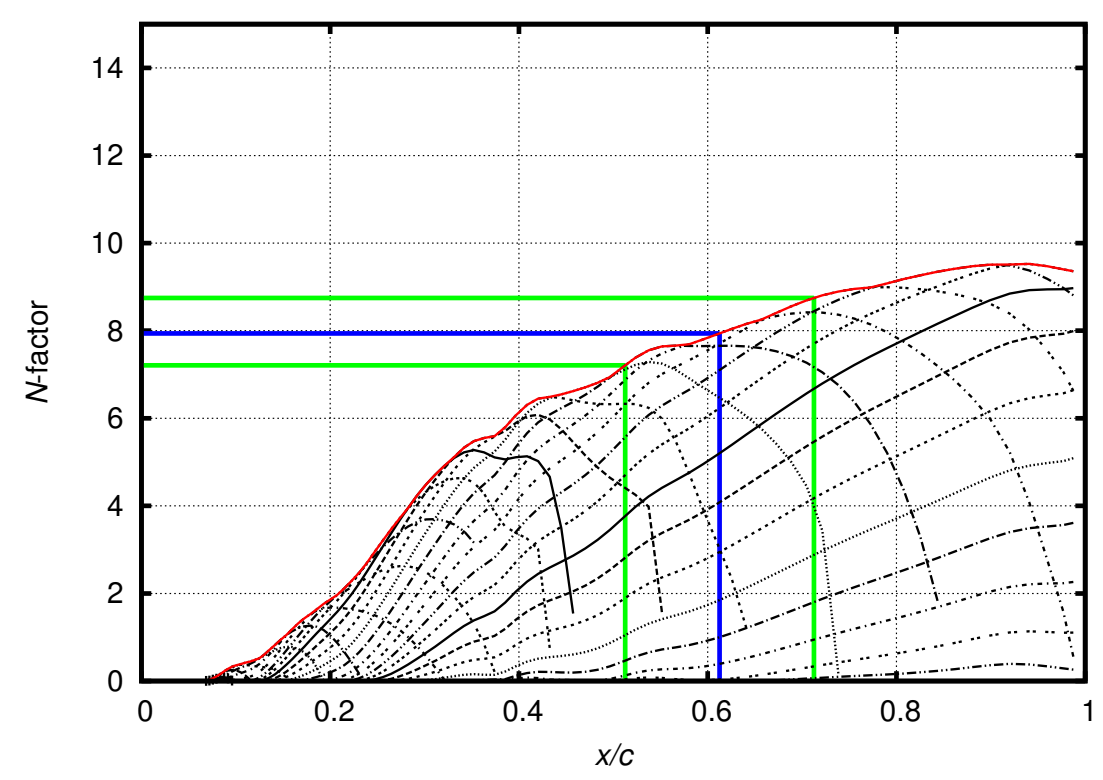

Figure 5.8: Compressible $N$-factors of Tollmien-Schlichting waves from compressible linear stability analysis as a function of normalized chordwise coordinate $x / c$, computed for the same test conditions as in Fig. 5.2. The black lines correspond to different amplified frequencies ranging from $f_{\min } \approx 6 \mathrm{kHz}$ to $f_{\max }=83 \mathrm{kHz}$. The envelope $N$-factor curve, indicating the maximal amplification, is marked by a red line. By reading off the maximum amplification at the transition location (blue line at $x / c \approx 0.6$ ) the compressible critical $N$-factor was determined $\left(N_{\text {comp }} \approx 8\right)$. When maximal possible variations of the transition location $( \pm 10 \%)$ are projected on the $N$-factor curve (green lines) an uncertainty of about $N \approx \pm 1$ can be estimated

instability and can cause transition to occur further upstream than in the adiabatic case (Boehman and Mariscalco, 1976; Costantini, 2016, Costantini et al., 2015b, 2016a, Fisher and Dougherty, 1982, Liepmann and Fila, 1947; Mack, 1984, Özgen, 2004; Schlichting and Gersten, 2000). However, the influence of a non-adiabatic surface temperature on the transition Reynolds number can be corrected (Costantini, 2016, Costantini et al., 2016a). To correct the measured non-adiabatic transition Reynolds number, $R e_{t r, n a w}^{\star}$, and calculate the adiabatic transition Reynolds numbers, $R e_{t r}^{\star}$, the following approximation is used, based on a linearized fit of the data from Costantini 2016 9 .

$$
\frac{R e_{t r, n a w}^{\star}}{\operatorname{Re}_{t r}^{\star}} \approx\left(\frac{T_{\text {naw }}}{T_{a w}}\right)^{\varphi} \approx \varphi \cdot \frac{T_{\text {naw }}}{T_{a w}}-\varphi+1
$$

Because the temperature difference between non-adiabatic and adiabatic model surface temperature, $\Delta T=T_{\text {naw }}-T_{a w}$, is small compared to the adiabatic surface temperature, with $\Delta T / T_{a w} \lesssim 0.05 \ll 1$ (Costantini, 2016), the linearization is valid for all Mach numbers and wall temperature ratios investigated in this study ${ }^{10}$ The exponent $\varphi$ was found to take different values, depending on Mach

\footnotetext{
${ }^{9}$ The term 'transition Reynolds number' will always refer to the adiabatic $R e_{t r}^{\star}$ in this paper.

${ }^{10}$ The observed trends of the linearized fit were confirmed by applying the $e^{N}$-method with a changing surface temperature distribution and comparing the determined transition locations under the assumption of a constant critical
} 


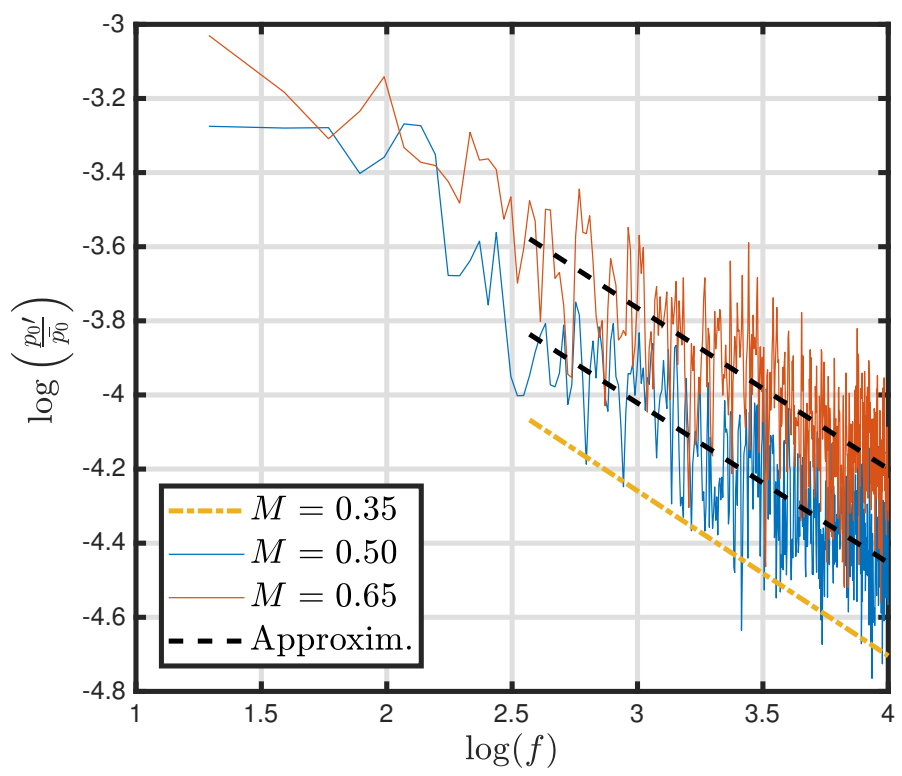

Figure 5.9: Power spectrum of total pressure fluctuations of DNW-KRG for different Mach numbers and a unit Reynolds number of $R e_{1}=30 \times 10^{6} \mathrm{~m}^{-1}$. The measured curves at $M=0.50$ (blue) and $M=0.65$ (red) were averaged from measurements at $15 \%$ and $50 \%$ test section width (see Fig. 4.37 of Koch (2004)). The decline of energy at frequencies between $f_{\text {min }} \approx 10^{2.6} \mathrm{~Hz} \approx 390 \mathrm{~Hz}$ and $f_{\max }=10^{4} \mathrm{~Hz}$ was approximated by Eq. 5.3, as shown by the dashed black lines. The function at $M=0.35$ (dashed yellow line) was calculated by Eq. I. D. Data is based on Koch (2004)

number, with $\varphi=-7$ (for $M=0.35$ ), $\varphi=-6$ (for $M=0.50$ ) and $\varphi=-3.5$ (for $M=0.65$ ) at the original PaLASTra model in DNW-KRG, which may be due to an increase in turbulence level with Mach number (Costantini, 2016).

\subsubsection{Influence of shape factor on transition Reynolds number}

It was found that the transition Reynolds number increases linearly with a more pronounced favourable pressure gradient, corresponding to a shape factor decrease, which is shown in Fig. 5.10 for $M=0.35$. The graphs for $M=0.50$ and $M=0.65$ are available in the Appendix. Consequently a linear function

$$
\operatorname{Re}_{t r}^{*}=h_{\mathrm{II}, 1} \cdot H_{12}+h_{\mathrm{II}, 0}
$$

with an intercept, $h_{\mathrm{II}, 0}$, and a slope, $h_{\mathrm{II}, 1}$, was fitted through each combination of Mach and Reynolds number (shown by solid lines in Fig. 5.10p. The coefficients $h_{\mathrm{II}, 0}$ and $h_{\mathrm{II}, 1}$ are summarized in Tables 5.2 and 5.3 , respectively. It can be seen that for a fixed value of $H_{12} \lesssim 2.6$ an increasing unit Reynolds number leads to an increasing transition Reynolds number (Fig. 5.10 and Table 5.2). Furthermore, an increasing unit Reynolds number leads to a decreasing slope $h_{\mathrm{II}, 1}$ (Fig. 5.10 and 
Table 5.1: Approximated coefficients $\alpha_{\mathrm{I}, M}$ and $\beta_{\mathrm{I}, M}$ of the double logarithmic relation between the spectral level of total pressure fluctuations and the frequency, calculated with Eq. I]

\begin{tabular}{lll}
\hline$M$ & $\alpha_{\mathrm{I}, M}$ & $\beta_{\mathrm{I}, M}$ \\
\hline 0.35 & -0.445 & -2.923 \\
0.45 & -0.441 & -2.770 \\
0.50 & -0.440 & -2.693 \\
0.55 & -0.438 & -2.616 \\
0.60 & -0.436 & -2.540 \\
0.65 & -0.434 & -2.462 \\
\hline
\end{tabular}

Table 5.3 11

Table 5.2: Intercepts, $h_{\mathrm{II}, 0}$, for measured unit Reynolds number and Mach number (see Eq. II

\begin{tabular}{llll}
\hline$R e_{1}^{*}$ & $M=0.35$ & $M=0.50$ & $M=0.65$ \\
\hline 17.5 & 63.14 & & \\
22.5 & & 86.26 & \\
30.0 & 105.15 & 103.96 & 103.09 \\
40.0 & 117.21 & 128.77 & 139.84 \\
50.0 & 148.00 & 171.05 & 141.63 \\
\hline
\end{tabular}

\subsubsection{Influence of unit Reynolds number $\left(R e_{1}^{\star}\right)$ on transition Reynolds number $\left(R e_{t r}^{\star}\right)$}

The approximation of the double logarithmic relation between the spectral level of total pressure fluctuations, $p^{\star}$, and the frequency (Eq. 5.3) motivates the use of a power law approach to approximate the transition Reynolds number as a function of the unit Reynolds number. It is known (Arnal, 1989) that in 'noisy' hypersonic wind tunnels a power relation exists with

$$
R e_{t r}^{\star} \sim\left(R e_{1}^{\star}\right)^{\alpha_{\mathrm{III}}}
$$

The exponent $\alpha_{\text {III }}$ is an empirical constant which was found to range between 0.1 and 0.6 for hypersonic flows (Arnal, 1989). To find the value of $\alpha_{\text {III }}$ for PaLASTra in DNW-KRG, the transition Reynolds number was plotted as a function of the unit Reynolds number, calculated with Eq. II] for different values of $H_{12}$ in a log-log plot (Fig. 5.11). In agreement with the hypersonic results, it was found in the present work that $\alpha_{\text {III }}$ takes values between 0.1 and 0.6 for accelerated flows, depending on Mach number and $H_{12}$ (also see Fig. A.3 in the Appendix). The dependence of $R e_{t r}^{*}$

\footnotetext{
${ }^{11}$ The determined intercepts $\left(h_{\mathrm{II}, 0}\right)$ and slopes $\left(h_{\mathrm{II}, 1}\right)$ can be approximated linearly as a function of unit Reynolds number to give a linear approximation of $R e_{t r}^{*}$ as a function of $R e_{1}^{*}$ and $H_{12}$ which is not shown here.
} 
Table 5.3: Slopes, $h_{\mathrm{II}, 1}$, for measured unit Reynolds number and Mach number (see Eq. II . For each slope the number of evaluated data points and the coefficient of determination of the linear fit, $R^{2}$, is given in brackets

\begin{tabular}{llll}
\hline$R e_{1}^{*}$ & $M=0.35$ & $M=0.50$ & $M=0.65$ \\
\hline 17.5 & -22.74 & & \\
& $(3 ; 0.962)$ & & \\
22.5 & & -31.43 & \\
& & $(3 ; 1.000)$ & \\
30.0 & -38.52 & -38.09 & -37.97 \\
& $(11 ; 0.992)$ & $(6 ; 0.999)$ & $(7 ; 0.997)$ \\
40.0 & -42.94 & -47.52 & -52.13 \\
& $(6 ; 0.971)$ & $(4 ; 0.999)$ & $(2 ; 1.000)$ \\
50.0 & -54.75 & -63.83 & -52.73 \\
& $(9 ; 0.981)$ & $(4 ; 1.000)$ & $(2 ; 1.000)$ \\
\hline
\end{tabular}

Table 5.4: Coefficients $h_{\text {III }, \alpha, i}$ with $i=0,1,2$ of the quadratic functions used to approximate $\alpha_{\text {III }}$ for the dependence of $R e_{t r}^{\star}$ on $R e_{1}^{\star}$ (Eq. IIIT)

\begin{tabular}{llll}
\hline$M$ & $h_{\mathrm{III}, \alpha, 2}$ & $h_{\mathrm{III}, \alpha, 1}$ & $h_{\mathrm{III}, \alpha, 0}$ \\
\hline 0.35 & -19.21 & 94.87 & -116.45 \\
0.50 & -25.77 & 128.25 & -159.09 \\
0.65 & -24.01 & 119.71 & -148.83 \\
\hline
\end{tabular}

on $R e_{1}^{*}$ was approximated via

$$
R e_{t r}^{*}=\left(R e_{1}^{*}\right)^{\alpha_{\mathrm{III}}} \cdot 10^{\beta_{\mathrm{III}}}
$$

where quadratic functions were used to approximate $\alpha_{\mathrm{III}}$ and $\beta_{\mathrm{III}}$ with $\alpha_{\mathrm{III}}=h_{\mathrm{III}, \alpha, 2} \cdot H_{12}^{2}+h_{\mathrm{III}, \alpha, 1}$. $H_{12}+h_{\mathrm{III}, \alpha, 0}$ and $\beta_{\mathrm{III}}=h_{\mathrm{III}, \beta, 2} \cdot H_{12}^{2}+h_{\mathrm{III}, \beta, 1} \cdot H_{12}+h_{\mathrm{III}, \beta, 0}$. A plot of the approximated quadratic functions is shown in Fig. 5.12 for $M=0.35$ (and in Fig. A.3 for $M=0.50$ and $M=0.65$ in the Appendix). The coefficients $h_{\mathrm{III}, \alpha, i}$ and $h_{\mathrm{III}, \beta, i}$ with $i=0,1,2$ are summarized in Tables 5.4 and 5.5 , respectively.

The dependency of the transition Reynolds number on the unit Reynolds number, approximated by the power law approach (Eq. III), is shown for each Mach number in Fig.5.13 for constant values of $H_{12}=2.51,2.54$ and 2.59, while the found dependency of $R e_{t r}^{\star}$ on $H_{12}$ is shown in Fig. 5.14 for $R e_{1}^{\star}=10,50$ and 80 . It can be seen that the transition Reynolds number increases with $R e_{1}^{\star}$ and decreases with $H_{12}$. It can also be seen that the transition Reynolds number decreases with increasing Mach number, mainly because of the increasing level of total pressure fluctuations with Mach number (Sect. 5.4.1). 


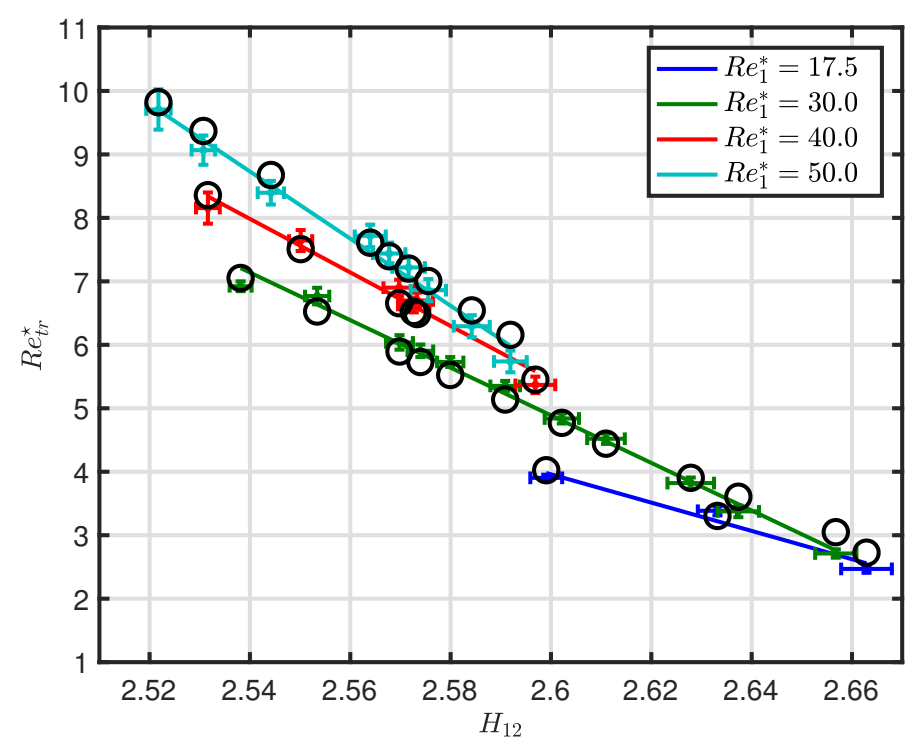

Figure 5.10: The transition Reynolds number as a function of the incompressible shape factor, $H_{12}$, for different unit Reynolds numbers at $M=0.35$. The vertical and horizontal error bars are RMS values of the transition location variation along the span (Fig. 5.3) and the chordwise shape factor approximation (Fig. 5.5, respectively. Black circles mark the calculated transition Reynolds numbers with the help of Eq. III

Table 5.5: Coefficients $h_{\mathrm{III}, \beta, i}$ with $i=0,1,2$ of the quadratic functions used to approximate $\beta_{\mathrm{III}}$ for the dependence of $R e_{t r}^{\star}$ on $R e_{1}^{\star}$ (Eq. IIIT)

\begin{tabular}{llll}
\hline$M$ & $h_{\mathrm{III}, \beta, 2}$ & $h_{\mathrm{III}, \beta, 1}$ & $h_{\mathrm{III}, \beta, 0}$ \\
\hline 0.35 & 10.77 & -54.50 & 69.11 \\
0.50 & 29.37 & -147.63 & 185.75 \\
0.65 & 25.20 & -127.19 & 160.89 \\
\hline
\end{tabular}

\subsubsection{Relation between frequency of most amplified T-S wave at the transition location $\left(f_{t r}\right)$ and the unit Reynolds number $\left(R e_{1}^{\star}\right)$}

The frequency of the most amplified T-S wave at the transition location, $f_{t r}$, was calculated using $L I L O$ as described in Sect. 5.3.3 ${ }^{12}$ In Fig. 5.15 $f_{t r}$ is plotted as a function of the shape factor, $H_{12}$, for different unit Reynolds numbers. To find the dependency of $f_{t r}$ on $R e_{1}^{\star}$ the following analysis was carried out: linear functions were used to approximate the relationship between $f_{t r}$ and $H_{12}$ with $f_{t r}=a_{R e_{1}^{\star}} \cdot H_{12}+b_{R e_{1}^{\star}}$ for each $R e_{1}^{\star}$. The average slope, $\bar{a}=\frac{1}{n} \Sigma_{R e_{1}^{\star}} a_{R e_{1}^{\star}}$, was determined and a linear function with the slope $\bar{a}$ was plotted through the mean values of $H_{12}$ and $f_{t r}$, for each $R e_{1}^{\star}$. These functions were used to find linear relations between $f_{t r}$ and $R e_{1}^{\star}$ for selected values of $H_{12}$.

\footnotetext{
${ }^{12}$ Frequencies of the most amplified waves calculated by incompressible and compressible stability theory were almost identical. In this case the incompressible calculations were used.
} 


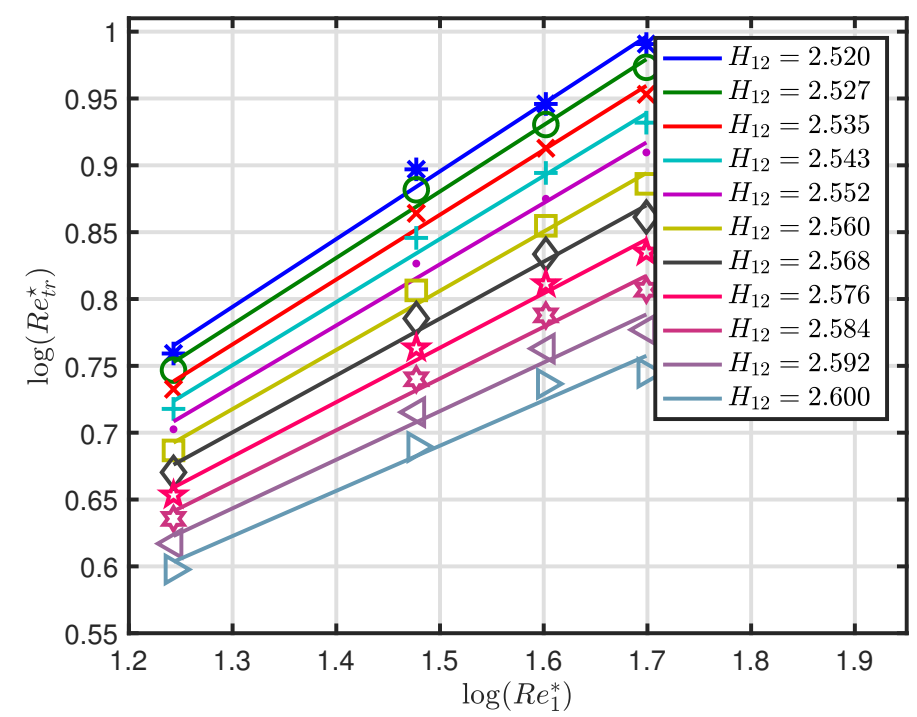

Figure 5.11: Transition Reynolds number as a function of unit Reynolds number in a double logarithmic plot for different values of $H_{12}$ at $M=0.35$. The symbols correspond to transition Reynolds numbers computed with Eq. II for $R e_{1}^{\star}=17.5,30,40$ and 50, corresponding to $\log \left(R e_{1}^{\star}\right) \approx 1.24,1.48,1.60$ and 1.70 , respectively. The approximations are shown by solid lines

The dependency of $f_{t r}$ on the $R e_{1}^{\star}$ can then be written as

$$
\begin{aligned}
f_{t r} & =\alpha_{\mathrm{IV}} \cdot R e_{1}^{\star}+\beta_{\mathrm{IV}} \\
& =\alpha_{\mathrm{IV}} \cdot R e_{1}^{\star}+\left(h_{\mathrm{IV}, \beta, 1} \cdot H_{12}+h_{\mathrm{IV}, \beta, 0}\right),
\end{aligned}
$$

where the coefficient $\beta_{\mathrm{IV}}$ was approximated linearly by $\beta_{\mathrm{IV}}=h_{\mathrm{IV}, \beta, 1} \cdot H_{12}+h_{\mathrm{IV}, \beta, 0}$. The coefficients $\alpha_{\mathrm{IV}}, h_{\mathrm{IV}, \beta, 0}$ and $h_{\mathrm{IV}, \beta, 1}$ are summarized in Table 5.6. The dependence of $f_{t r}$ on $R e_{1}^{*}$ is visualized in Fig. 5.16 for a fixed value of $H_{12}=2.59$. It can be seen that the unstable frequencies increase linearly with $R e_{1}^{\star}$. The linear increase of $f_{t r}$ with $R e_{1}^{\star}$ agrees with expectations from the definition of the dimensionless frequency, $F$, used in instability computations: $F=2 \pi \cdot f \cdot v / u_{e}^{2}$ Arnal et al. 1997; Schlichting and Gersten, 2000). This observation is also in agreement with previous results presented in the literature (Masad and Zurigat, 1994; Reed et al., 1996, Zurigat et al., 1992). The relationship given in Eq. IV can also be expressed with a dimensionless frequency,

$$
F_{t r}=\frac{2 \pi \cdot f_{t r}}{R e_{1} \cdot U_{\infty}}
$$

which leads to:

$$
F_{t r}=h_{\mathrm{IV}, \beta, 1, F} \cdot H_{12}+h_{\mathrm{IV}, \beta, 0, F}
$$

The resulting relationship (Eq. IVa) is shown in Fig. 5.17 and can be approximated by $F_{t r}=$ $1.140 \times 10^{-4} \cdot H_{12}-2.746 \times 10^{-4}$. Eq. IVa reveals no dependency on Mach and unit Reynolds numbers since they were integrated into $F_{t r}$. However, to perform a combination of the equations, as shown in the next section ( $\mathrm{Sec}$.5.4.6), it is necessary to express the unit Reynolds number 


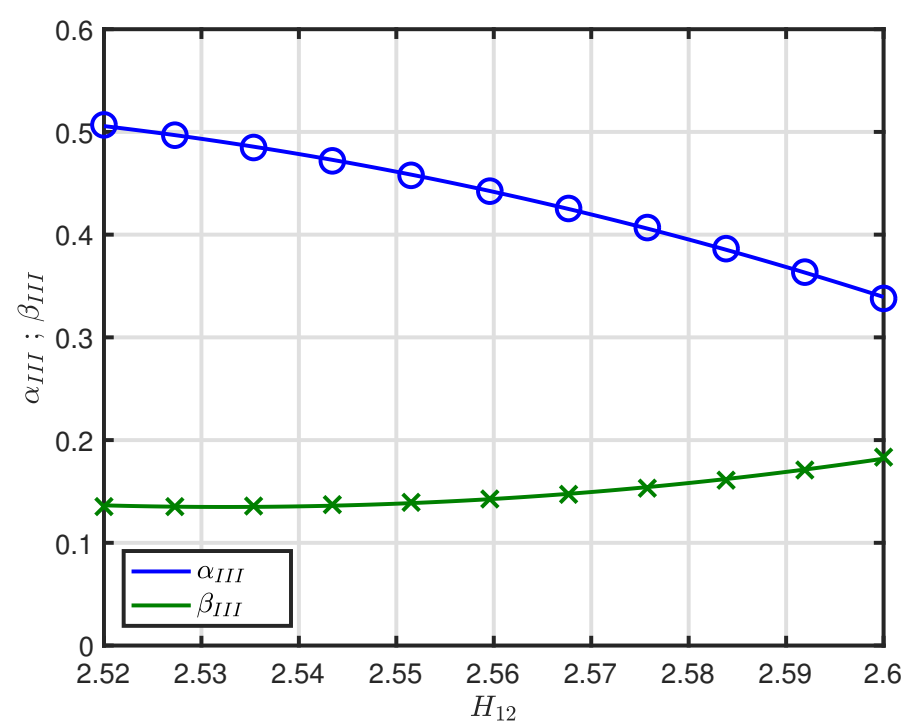

Figure 5.12: Coefficients $\alpha_{\text {III }}$ and $\beta_{\text {III }}$ as a function of $H_{12}$ at $M=0.35$ with approximations based on quadratic functions with $\alpha_{\mathrm{III}}=h_{\mathrm{III}, \alpha, 2} \cdot H_{12}^{2}+h_{\mathrm{III}, \alpha, 1} \cdot H_{12}+h_{\mathrm{III}, \alpha, 0}$ and $\beta_{\mathrm{III}}=h_{\mathrm{III}, \beta, 2} \cdot H_{12}^{2}+h_{\mathrm{III}, \beta, 1} \cdot H_{12}+h_{\mathrm{III}, \beta, 0}$

dependency explicitly, as done in Eq. IV, to eliminate $R e_{1}^{\star}$ in Eq. III (see Sec. 5.4.6.2, ${ }^{13}$ Therefore, dimensional frequencies will be used in the following.

Table 5.6: Coefficients $\alpha_{\mathrm{IV}}, h_{\mathrm{IV}, \beta, 1}$ and $h_{\mathrm{IV}, \beta, 0}$ of the linear functions used to approximate the dependence of $f_{t r}$ on $R e_{1}$ (Eq. IV)

\begin{tabular}{llll}
\hline$M$ & $\alpha_{\mathrm{IV}}$ & $h_{\mathrm{IV}, \beta, 1}$ & $h_{\mathrm{IV}, \beta, 0}$ \\
\hline 0.35 & 260.9 & 41024 & -103346 \\
0.50 & 338.5 & 72506 & -181870 \\
0.65 & 482.1 & 130938 & -333150 \\
\hline
\end{tabular}

\subsubsection{Combination of equations}

The equations labeled with roman numbers can be combined to express the transition Reynolds number as a function of the spectral level of total pressure fluctuations and $H_{12}$. Therefore, the unit Reynolds number is first expressed as a function of the frequency of the most amplified T-S wave at transition location. Then, the resulting function is inserted into Eq. III.

\footnotetext{
${ }^{13}$ This observation can also be understood from a physical standpoint, since the geometry and dimensions of the wind tunnel and wind tunnel model are relevant for the described analysis.
} 


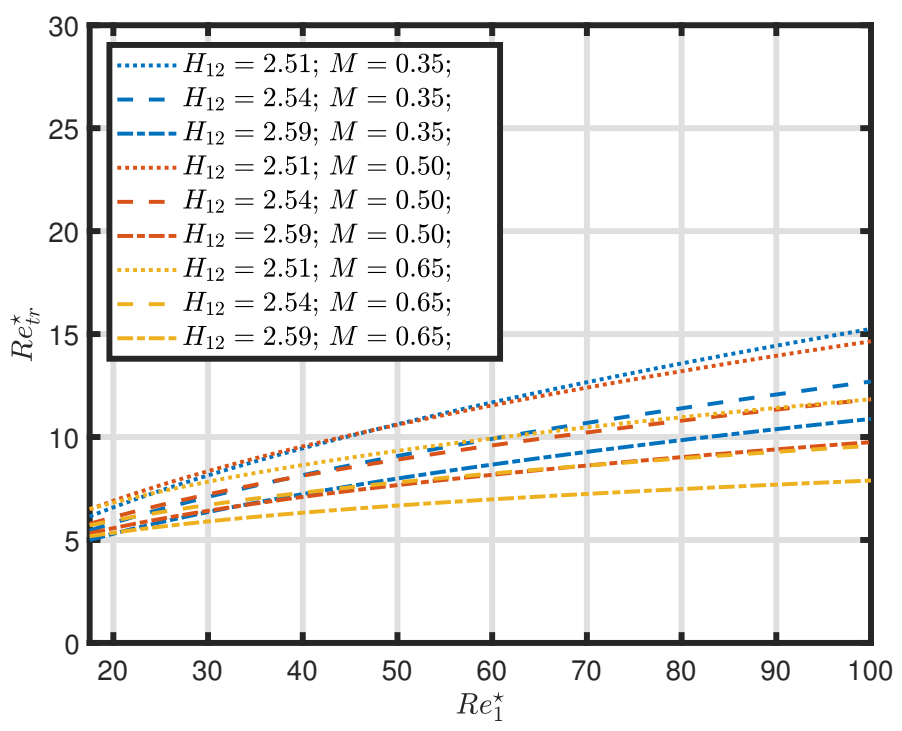

Figure 5.13: Transition Reynolds number, $R e_{t r}^{\star}$, as a function of unit Reynolds number, $R e_{1}^{\star}$, based on the power law approximation (Eq. III) with $H_{12}=2.51,2.54$ and 2.59

\subsubsection{Relationship between the unit Reynolds number $\left(R e_{1}^{\star}\right)$ and the spectral level of total pressure fluctuations $\left(p^{\star}\right)$}

The relation between unit Reynolds number, $R e_{1}^{\star}$, and the spectral level of total pressure fluctuations, $p^{\star}$, for the frequency of the most amplified T-S wave at transition location can be found by combination of Eqs. IV and I] with $f=f_{t r}$ and solving for $R e_{1}^{\star}$ :

$$
\begin{aligned}
\operatorname{Re}_{1}^{*} & =\frac{1}{\alpha_{\mathrm{IV}}}\left(f_{t r}-\beta_{\mathrm{IV}}\right) \\
& =\frac{1}{\alpha_{\mathrm{IV}}}\left(\left(\frac{p^{\star}}{10^{\beta_{\mathrm{I}}}}\right)^{\frac{1}{\alpha_{\mathrm{I}}}}-\beta_{\mathrm{IV}}\right) \\
& =\frac{1}{\alpha_{\mathrm{IV}}}\left(\left(\frac{p^{\star}}{10^{\beta_{\mathrm{I}}}}\right)^{\frac{1}{\alpha_{\mathrm{I}}}}-\left(h_{\mathrm{IV}, \beta, 1} \cdot H_{12}+h_{\mathrm{IV}, \beta, 0}\right)\right)
\end{aligned}
$$

\subsubsection{Transition Reynolds number $\left(R e_{t r}^{\star}\right)$ as a function of spectral level of total pressure fluctuations $\left(p^{\star}\right)$ and $H_{12}$}

To gain the transition Reynolds number as a function of spectral level of total pressure fluctuations Eq. $\mathrm{V}$ can be inserted into Eq. III:

$$
\begin{aligned}
\operatorname{Re}_{t r}^{*} & =\left(\operatorname{Re}_{1}^{*}\right)^{\alpha_{\mathrm{III}}} \cdot 10^{\beta_{\mathrm{III}}} \\
& =\left(\frac{1}{\alpha_{\mathrm{IV}}}\left(\left(\frac{p^{\star}}{10^{\beta_{\mathrm{I}}}}\right)^{\frac{1}{\alpha_{\mathrm{I}}}}-\beta_{\mathrm{IV}}\right)\right)^{\alpha_{\mathrm{III}}} \cdot 10^{\beta_{\mathrm{III}}}
\end{aligned}
$$

Eq. 5.7 is plotted as function of $H_{12}$ for a constant value of $p^{\star}=1 \cdot 10^{-4.5} \approx 3.162 \times 10^{-5}$ in Fig. 5.18, It can be seen from Fig. 5.18, that the calculated transition Reynolds numbers increases with increasing flow acceleration (corresponding to decreasing $H_{12}$ ). 


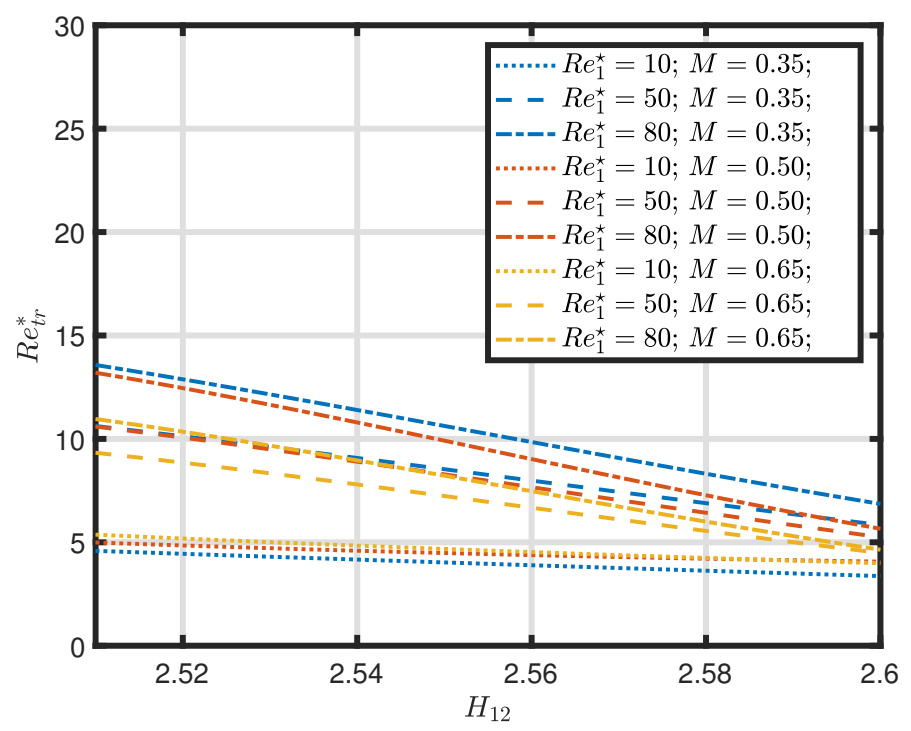

Figure 5.14: Transition Reynolds number, $R e_{t r}^{\star}$, as a function of shape factor, $H_{12}$, based on the power law approximation (Eq. III) with $R e_{1}^{\star}=10,50$ and 80

At a fixed spectral level of total pressure fluctuations $\left(p^{\star}=10^{-4.5}\right)$ the transition Reynolds numbers at different Mach numbers can finally be compared: It can be seen that the transition Reynolds numbers increase significantly with Mach number. This observation may be explained by compressibility effects, as predicted by linear stability theory (see Sect. 5.2.2, which were now isolated.

Transition Reynolds numbers, calculated by Eq. 5.7, are plotted as function of $p^{\star}$ with a constant value of $H_{12}=2.54 \mathrm{in} \mathrm{Fig.} \mathrm{5.19}$. It can be seen that for all $p^{\star}$, shown in Fig. 5.19, the calculated transition Reynolds numbers also show a significant increase with Mach number, which may also be accounted to compressibility effects.

\subsection{Compressible and incompressible critical $N$-factors and methods for correction}

The critical $\mathrm{N}$-factors were determined for all available data points with compressible and incompressible stability theory and shown in the top graphs of Figs. 5.20 and 5.21, respectively. It can be seen that in both cases the determined critical $N$-factors generally decrease with decreasing $H_{12}$.

For comparison of the Mach number influence on the determined critical $\mathrm{N}$-factors, the $\mathrm{N}$ factors of the first tunnel entry were analyzed separately for each Mach number and their mean and standard deviation are compared in Table 5.7. Furthermore, the determined mean critical $N$-factors are plotted as a function of $M$ in Fig. 5.23. While the compressible critical $N$-factors decrease over the whole Mach number range, the incompressible critical $N$-factors remain almost constant for $M=0.50$ and 0.65 . Therefore the standard deviation over all Mach numbers of the compressible critical $N$-factor is larger than in the incompressible case (see Table 5.7 last row). However, when the Mach numbers are analyzed separately, the compressible critical $N$-factors 


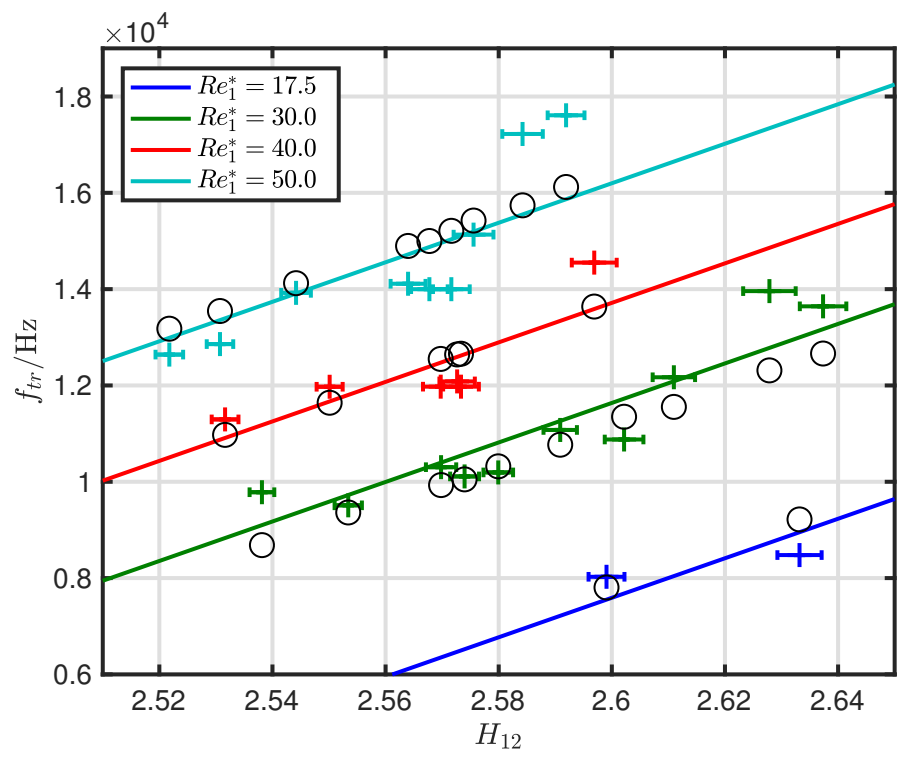

Figure 5.15: Frequency of the most amplified T-S wave at the transition location as a function of $H_{12}$ for different values of unit Reynolds numbers at $M=0.35$. The same analysis has been carried out and is presented in Fig. A.4 of the Appendix for $M=0.50$ and $M=0.65$. Error bars are RMS values of the $H_{12}$ approximation as shown in Fig. 5.5 . For comparison the black circles show the approximated value of $f_{t r}$ with the help of Eq. IV

exhibit a smaller variation (standard deviation, $\sigma$, and maximal variation, $\Delta N=N_{\max }-N_{\min }$ ) than in the incompressible case.

To capture the expected increase in initial disturbance amplitude by an increased spectral level of total pressure fluctuations, a correction method of the critical $N$-factor was developed. The correction method is founded on three basic assumptions of linear stability theory and receptivity (van Ingen, 2008):

1. The critical $N$-factor relates the starting amplitude of the T-S wave, $A_{0}$, to the amplitude of the T-S wave at which transition occurs, $A_{T S, t r}$ van Ingen, 2008):

$$
N=\ln \left(\frac{A_{T S, t r}}{A_{0}}\right)=\ln \left(A_{T S, t r}\right)-\ln \left(A_{0}\right)
$$

2. The Tollmien-Schlichting waves become unstable and lead to transition at a certain amplitude. (Often an amplitude of about $1 \%$ of the freestream velocity, $A_{T S, t r} \approx 0.01 \cdot U_{\infty}$ is assumed (Herbert, 1997; Würz et al., 2012b)

3. The receptivity process remains unchanged and a linear relation between initial amplitude of the T-S wave, $A_{0}$, and the spectral level of total pressure fluctuations, $p^{\star}$, exists with $A_{0} \sim p^{\star}$ (Fuciarelli et al., 2000; Lin et al., 1992; Saric and White, 1998; Saric et al., 1999) 


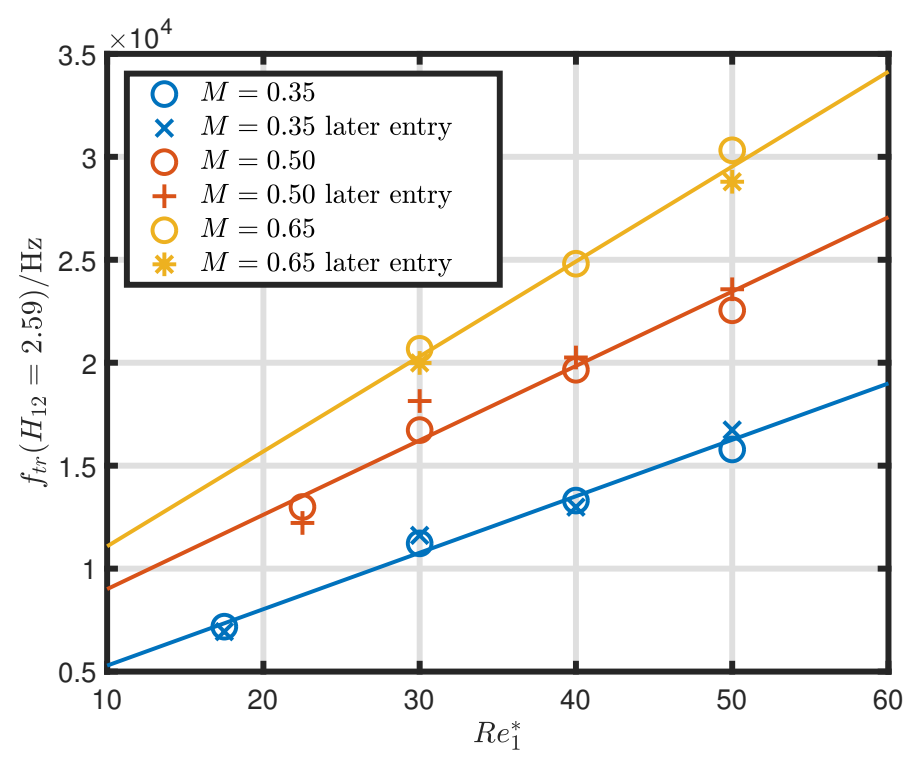

Figure 5.16: Frequency of the most amplified T-S wave at the transition location as a function of unit Reynolds number for a constant value of $H_{12}=2.59$ at $M=0.35, M=0.50$ and $M=0.65$. The data of later tunnel entries, which was also used for approximation, is in agreement with data of the first tunnel entry

\subsubsection{Correction of the critical $N$-factors with the spectral level of total pressure fluctuations ( $p^{\star}$-correction)}

A corrected critical $N$-factor was defined for an arbitrary reference spectral level of $p_{\text {ref }}^{\star}=$ $1.5 \times 10^{-5}$, in parallel with Eq. 5.8 .

$$
N_{p^{\star}}=\ln \left(\frac{A_{T S, t r}}{A_{0, p^{\star}}}\right)=\ln \left(A_{T S, t r}\right)-\ln \left(A_{0, p^{\star}}\right)
$$

To gain a reference critical $N$-factor, Eq. 5.8 was subtracted from Eq. 5.9. The reference critical $N$-factor, $N_{p^{\star}}$, was then calculated under the assumption of a linear relationship between $A_{0}$ and $p^{\star}$, with:

$$
N_{p^{\star}}=\ln \left(\frac{A_{0}}{A_{0, p^{\star}}}\right)+N=\ln \left(\frac{p^{\star}}{p_{r e f}^{\star}}\right)+N
$$

The spectral level $\left(p^{\star}\right)$ corresponding to the critical $N$-factor was determined by inserting the frequency of the most amplified T-S wave at transition location and the Mach number into Eq. I.

It can be seen from Fig. 5.23 and Table 5.7 that the dependency of the corrected compressible critical $N$-factor $\left(N_{c o m p, p^{\star}}\right)$, exhibits much less variation with Mach number than the compressible critical $N$-factor without correction. Table 5.7 also shows that the corrected critical $N$-factors ( $N_{\text {comp }, p^{\star}}$ and $N_{i n c, p^{\star}}$ ) exhibit smaller standard deviations and maximal variations than the compressible and incompressible $N$-factors $\left(N_{\text {comp }}\right.$ and $N_{i n c}$ ). However, it can also be seen from the plots in the middle of Figs. 5.20 and 5.21 that the critical $N$-factors corrected by the corresponding spectral level $\left(N_{c o m p, p^{\star}}\right.$ and $N_{i n c, p^{\star}}$ ) still show a dependency on $H_{12}$. Therefore the influence of acoustic disturbances is analyzed in the next section. 


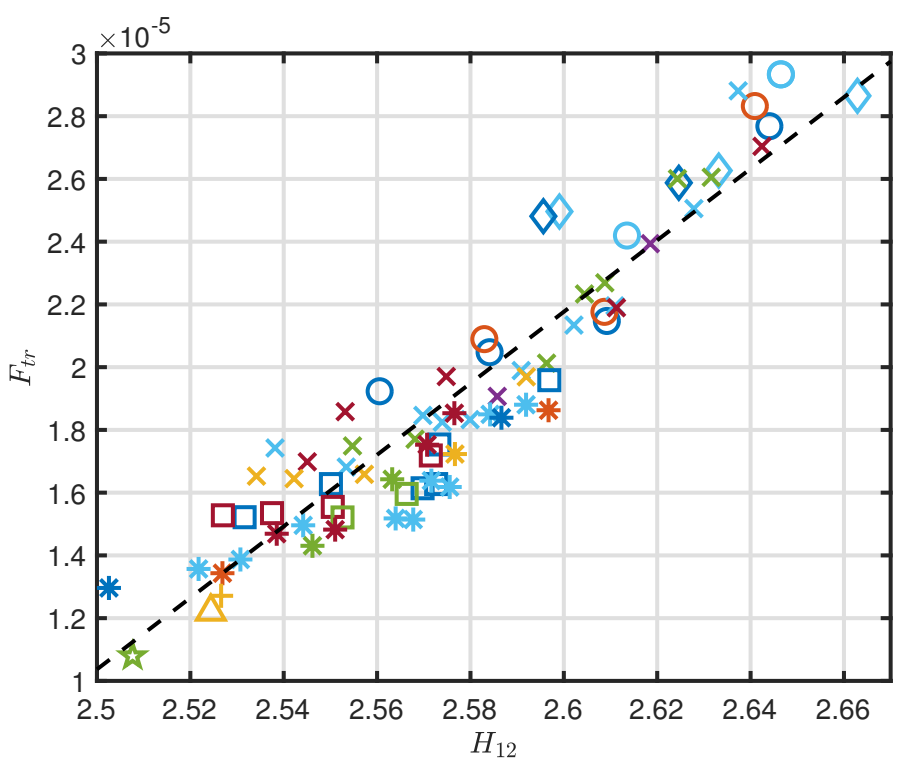

Figure 5.17: Dimensionless frequency $F_{t r}$ of the most amplified T-S wave at the transition location as a function of $H_{12}$ for all available Mach $(M=0.35$ to 0.77$)$ and unit Reynolds numbers $\left(R e_{1}=17.5 \times 10^{6} \mathrm{~m}^{-1}\right.$ to $\left.80 \times 10^{6} \mathrm{~m}^{-1}\right)$. The legend is shown in Fig. 5.22

\subsubsection{Correction of the determined critical $N$-factors by receptivity dependency of acoustic disturbances on incidence angles (C-correction)}

Random fluctuations can be decomposed into three distinct modes: vorticity, sound and entropy (Kovasznay, 1953; Michel and Froebel, 1988). While the influence of total pressure fluctuations which correspond to non-isentropic variations that constitute the entropy mode, acoustic disturbances, which correspond to isentropic fluctuations, will be investigated in this section. In the following, the dependence of receptivity on the incidence angle of acoustic disturbances is used to correct the determined critical $\mathrm{N}$-factor. The correction method is founded on the following four assumptions:

1. The acoustic disturbances remain constant for the investigated ranges of frequency, Mach and Reynolds numbers

2. The receptivity of acoustic disturbances depends strongly on the incidence angle by which disturbances are coupled into the boundary layer (Erturk and Corke, 2001; Fuciarelli et al. 2000; Goldstein and Hultgren, 1989; Haddad and Corke, 1998; Hammerton and Kerschen, 1996, Heinrich et al., 1988). The receptivity coefficient, $C$, is defined as the ratio of T-S wave amplitude to acoustic wave amplitude. It increases with increasing incidence angle, $\theta$, and can be approximated linearly for small angles (Heinrich et al., 1988), with a slope, $c$, via

$$
C=1+c \cdot \theta
$$




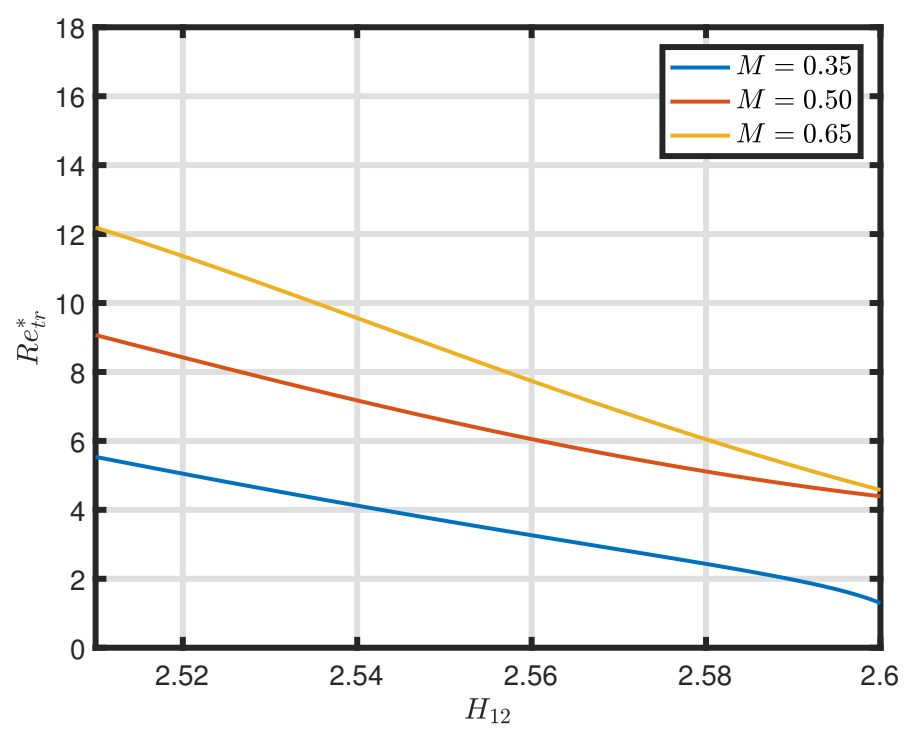

Figure 5.18: Transition Reynolds number, calculated by Eq. 5.7, is plotted as a function of $H_{12}$ for a constant value of $p^{\star}=10^{-4.5} \approx 3.162 \times 10^{-5} . R e_{t r}^{\star}$ decreases with increasing $H_{12}$ and $R e_{t r}^{\star}$ increases with $M$

3. Acoustic disturbances in the wind tunnel are assumed to originate mainly from the storage tube. Therefore, they can be assumed to be aligned with the flow direction. Hence, the incidence angle, $\theta$, is assumed to have the same magnitude as the angle-of-attack of the model, $\alpha$, with $\theta=|\alpha|$

4. A constant value of $c=0.1818 /{ }^{\circ}$ is assumed, which is based on an approximation of results by Heinrich et al. (1988) for a flat plate with sharp leading edge at $M=0.1^{14}$

Based on these assumptions a receptivity corrected $N$-factor, $N_{C}$, can be derived in parallel with Eq. 5.10. Under the assumption of a reference receptivity coefficient, $C_{r e f}=1$, these assumptions lead to the following correction:

$$
N_{C}=\ln \left(\frac{C}{C_{r e f}}\right)+N=\ln (1+c \cdot|\alpha|)+N
$$

The $N$-factor corrections of total pressure fluctuations (Eq. 5.10) and acoustic disturbances (Eq. 5.12) can be combined to calculate corrected $N$-factors, $N_{p^{\star}, C}$, via:

$$
N_{p^{\star}, C}=\ln \left(\frac{p^{\star}}{p_{\text {ref }}^{\star}}\right)+\ln (1+c \cdot|\alpha|)+N
$$

The results are compared in Figs. 5.20, 5.21 (bottom) and Table 5.7. By correcting the angular dependency of the receptivity coefficient, the dependency of the determined critical $\mathrm{N}$-factors

\footnotetext{
${ }^{14}$ The found receptivity coefficients vary strongly, depending on nose shape, nose radius and Mach number (eg. Heinrich et al. (1988) and Shahriari et al. (2016). Therefore, the assumed value of $c \approx 0.2$ can only be an approximation, which is used to investigate the plausibility of the investigated trends. The main goal of the described $C$-correction method is to illustrate the working principle, which can be used in the future for other investigations with more appropriate values of $c$. For further discussion on the influence of $c$, see also Sect. 5.7.3.2
} 


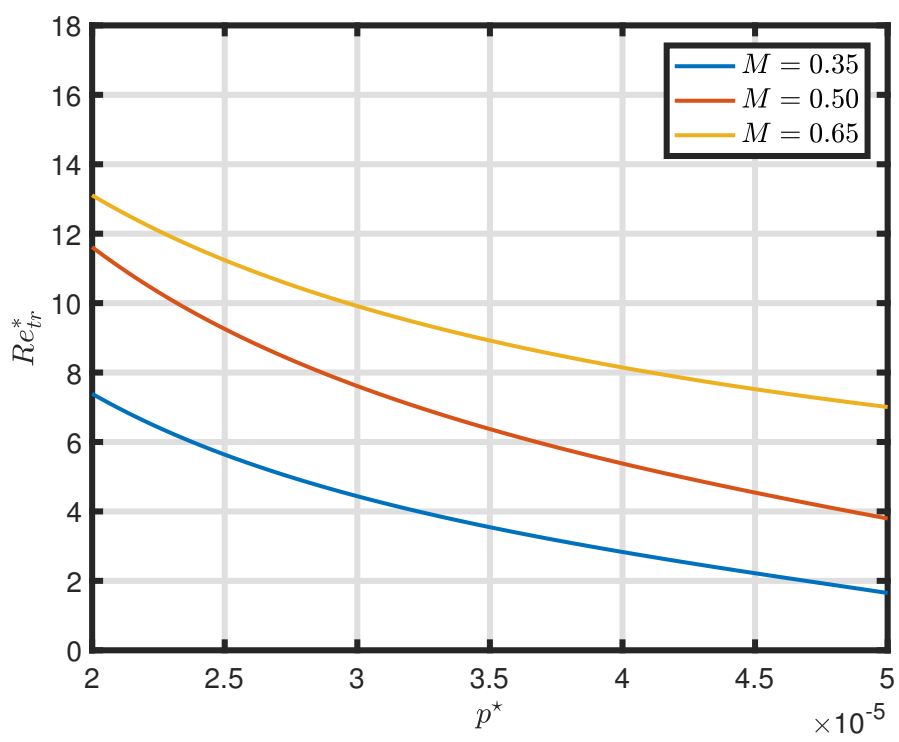

Figure 5.19: Transition Reynolds number, calculated by Eq. 5.7, is plotted as function of $p^{\star}$ for a constant value of $H_{12}=2.54$. $R e_{t r}^{\star}$ decreases with increasing $p^{\star}$ and $R e_{t r}^{\star}$ increases with $M$

on $H_{12}$ is reduced but not completely eliminated. It can be seen that the corrected compressible $N$-factors, $N_{\text {comp }, p^{\star}, C}$, show the smallest maximal variations.

\subsection{Uncertainties and repeatability}

This section contains four parts: In the first part, uncertainties of the measured variables are described (Sect. 5.6.1). In the second part, uncertainties of the transition Reynolds number analysis are discussed (Sect. 5.6.2), while, in the third part, uncertainties in the analysis of critical $N$-factors are specified (Sect. 5.6.3). In the last part of this section, the repeatability for different wind tunnel entries is discussed (Sect. 5.6.4).

\subsubsection{Uncertainties of measured parameters}

The influence of measurement uncertainties of the flow parameters (unit Reynolds number, Mach number, freestream and wall temperatures) are so small that they can be neglected in the current analysis. To quantify the uncertainties in transition Reynolds number and shape factor, their root mean square (RMS) were determined (Sect. 5.3.2 and 5.3.3), leading to $\left(R e_{t r}^{\star}\right)_{\mathrm{RMS}}=0.5$ and $\left(H_{12}\right)_{\mathrm{RMS}}=0.01$, which corresponds to relative errors of about $5 \%$ and $0.5 \%$, respectively. Based on Figs. 5.9 and 5.15, the uncertainties in the spectral level $\left(p^{\star}\right)$ and in the most amplified frequency at transition location $\left(f_{t r}\right)$ are estimated to be about $10 \%$. 

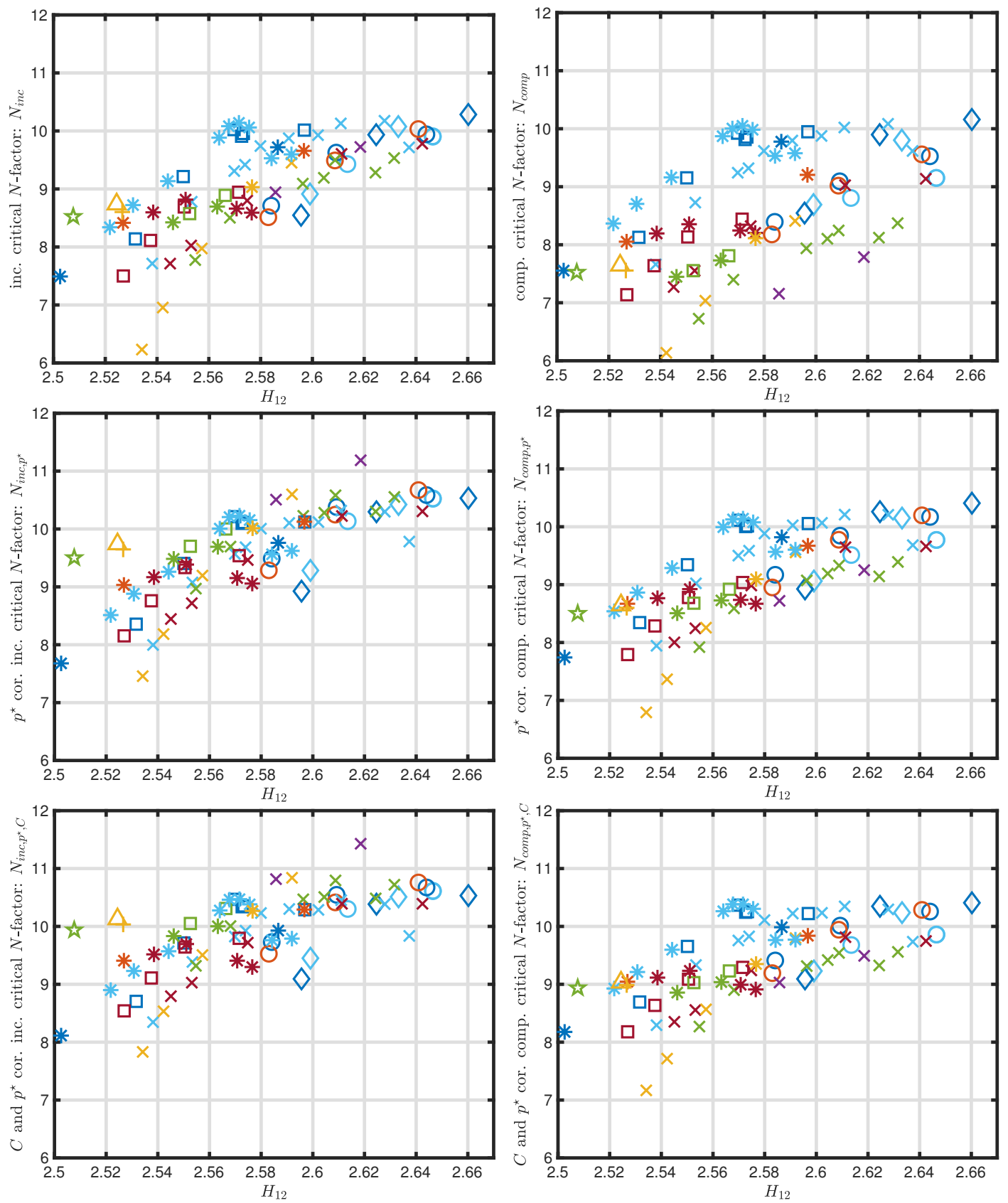

Figure 5.20: Incompressible critical $N$-factors, Figure 5.21: Compressible critical $N$-factors,

$N_{i n c}, N_{i n c, p^{\star}}$ and $N_{i n c, p^{\star}, C}$ (from top to bottom) as a function of $H_{12}$ at all available Mach $(M=$ 0.35 to 0.77$)$ and unit Reynolds numbers $\left(R e_{1}=17.5 \times 10^{6} \mathrm{~m}^{-1}\right.$ to $\left.80 \times 10^{6} \mathrm{~m}^{-1}\right)$. The legend is shown in Fig. 5.22
$N_{\text {comp }}, N_{\text {comp }, p^{\star}}$ and $N_{\text {comp }, p^{\star}, C}$ (from top to bottom) as a function of $H_{12}$ at all available Mach $(M=$ 0.35 to 0.77$)$ and unit Reynolds numbers $\left(R e_{1}=17.5 \times 10^{6} \mathrm{~m}^{-1}\right.$ to $\left.80 \times 10^{6} \mathrm{~m}^{-1}\right)$. The legend is shown in Fig. 5.22 


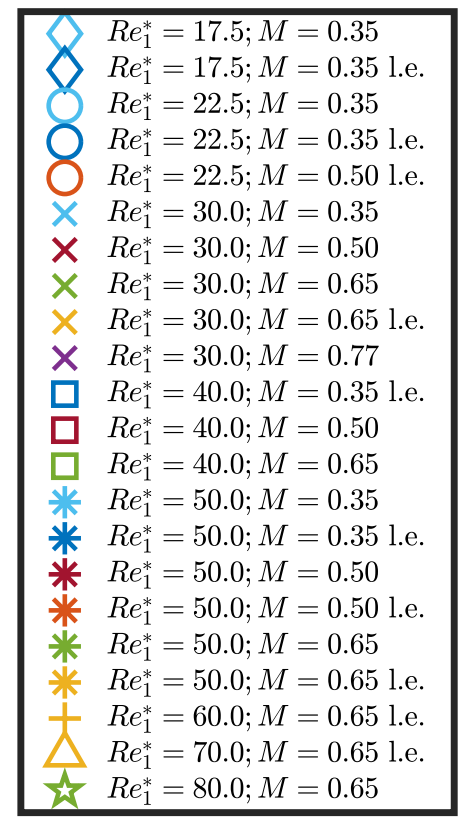

Figure 5.22: Legend for all available Mach $(M=0.35$ to 0.77$)$ and unit Reynolds numbers $\left(R e_{1}=\right.$ $17.5 \times 10^{6} \mathrm{~m}^{-1}$ to $80 \times 10^{6} \mathrm{~m}^{-1}$ plotted in Figs. 5.6, 5.17, 5.20 and 5.21

\subsubsection{Uncertainties in the transition Reynolds number analysis}

In the relation between the unit Reynolds number and the transition Reynolds number (Sect. 5.4.4) where a power law is used $\left(R e_{t r}^{*}=\left(R e_{1}^{*}\right)^{\alpha_{\text {III }}} \cdot 10^{\beta_{\text {III }}}\right.$ with $\alpha_{\text {III }}$ and $\beta_{\text {III }}$ approximated by quadratic functions), variations are about $5 \%$, as shown in Fig. 5.12.

Further uncertainties are induced by the calculated transition Reynolds number, based on the correction of the non-adiabatic surface temperature (Sect.5.4.2): the used coefficients for correction were measured with the original PaLASTra model which induces additional pressure fluctuations inside the test section. The influence of these pressure fluctuations on the transition location is too complex to be quantified, so that the approximations should be checked with the modified PaLASTra model in the future.

Due to large uncertainties in the quantification of (a) nose bluntness and (b) receptivity effects, they were not included in the transition Reynolds number analysis. These effects may lead to further uncertainties in the determined transition Reynolds numbers, which are difficult to estimate. Based on the above discussions, a rigorous error calculus is not possible for the derived equation (Eq. 5.7), but may be assumed to be in the order of about $10 \%$.

The derived function (Eq. 5.7p can also be extrapolated to other spectral levels and shape factors; however, this should be done with great caution as it is not based on measurements. Furthermore, it should be noted that the power spectrum of total pressure fluctuations was only measured up to $10 \mathrm{kHz}$ and extrapolated for higher frequencies. In this context it would of course be useful to extend the measurement to larger measurement ranges, or repeat them with a higher accuracy. Nevertheless, the presented results are an important step to describe the Mach number and unit Reynolds number effect quantitatively. 


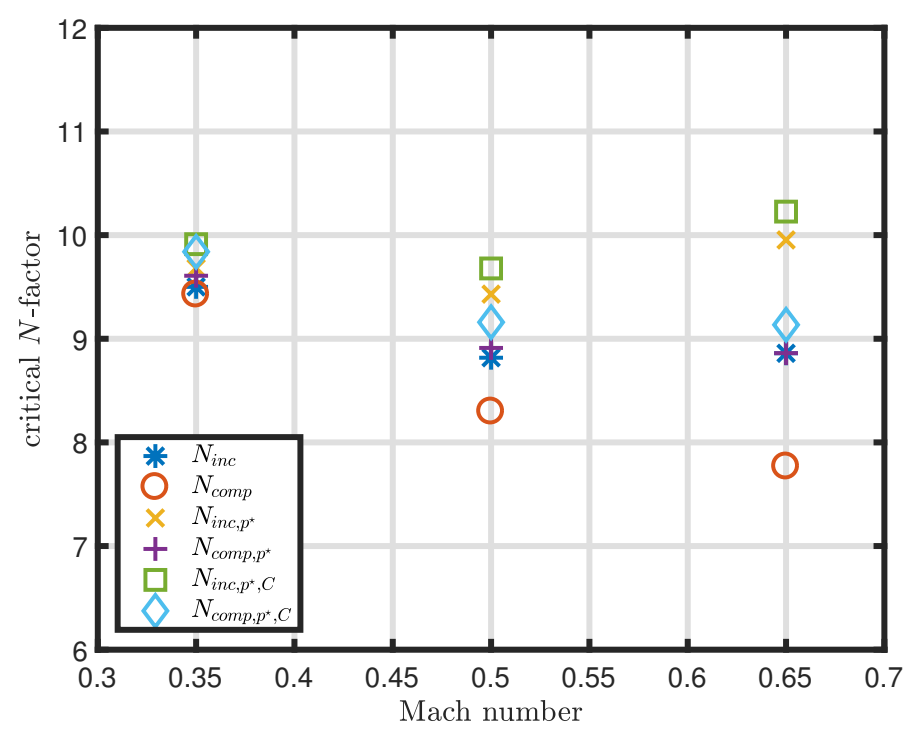

Figure 5.23: Comparison of determined mean critical $N$-factors as a function of Mach number. Compressible critical $N$-factors, $N_{\text {comp }}$, decrease with increasing Mach numbers (data from Table 5.7p

Table 5.7: Determined critical $N$-factors by compressible theory $\left(N_{\text {comp }}\right)$, incompressible theory $\left(N_{\text {inc }}\right)$, corrected spectral level of total pressure fluctuations $\left(N_{c o m p, p^{\star}}\right.$ and $\left.N_{i n c, p^{\star}}\right)$ and additionally corrected receptivity of acoustic disturbances $\left(N_{c o m p, p^{\star}, C}\right.$ and $\left.N_{i n c, p^{\star}, C}\right)$ with their standard deviations ( $\sigma$, behind the \pm sign) and the maximal variation of the $N$ factor $\left(\Delta N=N_{\max }-N_{\min }\right)$ in round brackets. ${ }^{\#}$ The row labeled with 'all', refers to all available critical $N$-factors, as shown in Figs. 5.20 and 5.21

\begin{tabular}{llll}
\hline$M$ & $N_{\text {inc }}$ & $N_{\text {comp }}$ & $N_{\text {inc, } p^{\star}}$ \\
\hline 0.35 & $9.50 \pm 0.68(2.46)$ & $9.43 \pm 0.65(2.42)$ & $9.68 \pm 0.65(2.43)$ \\
0.50 & $8.82 \pm 0.75(2.41)$ & $8.30 \pm 0.65(2.01)$ & $9.43 \pm 0.74(2.37)$ \\
0.65 & $8.86 \pm 0.53(1.76)$ & $7.77 \pm 0.47(1.65)$ & $9.95 \pm 0.49(1.61)$ \\
all $^{\#}$ & $8.99 \pm 0.95(4.29)$ & $8.50 \pm 1.14(4.90)$ & $9.60 \pm 0.84(4.06)$ \\
\hline \hline$M$ & $N_{\text {comp }, p^{\star}}$ & $N_{\text {inc }, p^{\star}, C}$ & $N_{\text {comp }, p^{\star}, C}$ \\
\hline 0.35 & $9.61 \pm 0.62(2.27)$ & $9.91 \pm 0.59(2.16)$ & $9.84 \pm 0.57(2.09)$ \\
0.50 & $8.91 \pm 0.63(1.98)$ & $9.68 \pm 0.64(2.07)$ & $9.16 \pm 0.54(1.68)$ \\
0.65 & $8.86 \pm 0.43(1.47)$ & $9.91 \pm 0.60(2.45)$ & $9.13 \pm 0.38(1.29)$ \\
all $^{\#}$ & $9.11 \pm 0.87(4.02)$ & $9.85 \pm 0.76(3.81)$ & $9.36 \pm 0.78(3.53)$ \\
\hline
\end{tabular}




\subsubsection{Uncertainties in the critical $N$-factor analysis}

Even more difficult is the quantification of uncertainties of the determined critical $N$-factors, because additionally uncertainties in the receptivity process (discussed in Sect. 5.7.3.2) and linear stability analysis would have to be considered.

Due to the many unknown factors the description of uncertainties shall be restricted to the found variations in $N$-factors. When the maximum possible variations of the transition location $( \pm 10 \%)$ are projected onto the $N$-factor curve, an uncertainty of about $N= \pm 1$ can be estimated (Fig. 5.8). Hence, the value of \pm 1 can be seen as a meaningful estimation for the uncertainty of the determined critical $N$-factors.

At a fixed value of $H_{12}$ a scatter of the determined critical $N$-factors between $\Delta N=3$ (without corrections) and $\Delta N=1.5$ (with corrections applied) was found. When the dependence on $H_{12}$ is included and all available data points are analyzed, variations between $\Delta N=4.9$ (without corrections) and $\Delta N=3.5$ (with corrections applied) are found.

In general, it should be emphasized that the scatter of the found $N$-factors is smaller than in most previous investigations conducted at high Reynolds numbers (Schrauf, 1994, 2000, 2005; Schrauf et al., 1996, 1998).

\subsubsection{Repeatability of wind tunnel entries}

As mentioned in Sect. 5.3, the modified PaLASTra model was repeatedly tested in DNW-KRG in six different measurement campaigns over a time span of two years. Between the second entry and later entries the test section of the wind tunnel was consolidated and slightly modified. Also the PaLASTra model was disassembled several times within this time range. However, it can be seen from Figs. 5.6, 5.17, 5.20 and 5.21, that in general a good repeatability of the results can be found when a sufficient amount of data points are acquired.

\subsection{Discussion of results}

In this study the influence of unit Reynolds number, Mach number and pressure gradient (quantified by the incompressible shape factor) on the transition Reynolds number was investigated; this will be summarized and discussed in the first part of this section (Sect. 5.7.1). Furthermore, a correction of the determined compressible and incompressible critical $N$-factors was carried out as is summarized and discussed in the second part of this section (Sect. 5.7.2).

\subsubsection{Factors influencing transition Reynolds number}

\subsubsection{Unit Reynolds number effect}

A concise summary of the observed increase of $R e_{t r}$ with $R e_{1}$ was given by Arnal (1989), who stated that "the relative motion of the environmental disturbances spectrum and of the linearly unstable frequency range can give rise to a strong unit Reynolds number effect." Arnal also noted that the cause for the unit Reynolds number effect may be a combined response to many factors, 
such as changes in (a) nose bluntness, (b) the receptivity of the model boundary layer, (c) the freestream disturbance spectrum and (d) the range of potentially unstable frequencies (Arnal, 1989). Based on the factors (c) and (d), Stetson et al. (1986) gave a good schematic explanation of the unit Reynolds number effect with the following words: "With increasing unit Reynolds number, generally it is expected that the frequencies of the most unstable boundary layer disturbances will increase more rapidly than the upper frequency excitation limit of the environment. The result is that disturbances above some frequency may not grow even though they are unstable."

Founded on the same approach, the unit Reynolds number effect was investigated in this work. However, instead of assuming an upper frequency excitation limit of the environment (like Stetson et al. (1986)), in this investigation the excitation intensity of the environment is quantified by the spectral level of total pressure fluctuations. Furthermore, it is not assumed that disturbances above some frequency will not grow at all (as done by Stetson et al. (1986)) instead it is assumed that the initial amplitude of disturbances in the boundary layer varies, depending on Mach number and frequency. With this approach it was not only possible to give a qualitative explanation of the unit Reynolds number effect, but also to describe it quantitatively. To achieve this quantitative description, two approximations were carried out in this paper:

1. The spectral level of total pressure fluctuations was approximated as a function of frequency and Mach number (Eq. I)

2. The dependence of $R e_{t r}^{\star}$ on $R e_{1}^{\star}$ was approximated with the help of a power law approach (Eq. III)

The power law used to quantify the dependence of $R e_{t r}^{\star}$ on $R e_{1}^{\star}$ is given by $R e_{t r}^{*}=\left(R e_{1}^{*}\right)^{\alpha_{\mathrm{III}}} \cdot 10^{\beta_{\mathrm{III}}}$ (Eq. III). The exponents found for accelerated flows $\left(\alpha_{\mathrm{III}}=0.1\right.$ to 0.6$)$, agree with findings in hypersonic wind tunnels Arnal, 1989). The coefficients $\alpha_{\text {III }}$ and $\beta_{\text {III }}$ were calculated with the help of quadratic approximations, $\alpha_{\mathrm{III}}=h_{\mathrm{III}, \alpha, 2} \cdot H_{12}^{2}+h_{\mathrm{III}, \alpha, 1} \cdot H_{12}+h_{\mathrm{III}, \alpha, 0}$ and $\beta_{\mathrm{III}}=h_{\mathrm{III}, \beta, 2} \cdot H_{12}^{2}+$ $h_{\mathrm{III}, \beta, 1} \cdot H_{12}+h_{\mathrm{III}, \beta, 0}$, for each Mach number separately.

\subsubsection{Mach number effect}

As discussed in Sect. 5.2.2, a stabilizing effect of compressibility on boundary layer transition is predicted by linear stability theory. However, an increased initial amplitude of the T-S waves, due to an increased freestream disturbance level, prohibits a direct comparison of transition Reynolds numbers measured at different Mach numbers (Arnal, 1989). To allow a comparison of transition Reynolds numbers measured at different Mach numbers, which have a different freestream disturbance level, an additional third approximation was carried out (Sects. 5.4.5 and 5.7.1.1:

3. The frequency of the most amplified T-S wave at the transition location was approximated as a function of $H_{12}$ and $R e_{1}^{\star}(\mathrm{Eq} . \mathrm{IV}$ ).

By combination of the above equations (labeled with roman numbers) the unit Reynolds number was eliminated, and the transition Reynolds number was expressed as functions of the freestream 
disturbance environment, characterized by $p^{\star}$, and by the shape factor, $H_{12}$ (Eq. 5.7). For a comparison of different Mach numbers, a fixed spectral level of total pressure fluctuations $\left(p^{\star}=\right.$ $\left.10^{-4.5}\right)$ or a fixed shape factor $\left(H_{12}=2.54\right)$ were entered into Eq. 5.7 and visualized in Figs. 5.18 and 5.19. Within the measurement range $\left(2 \times 10^{-5} \leq p^{\star} \leq 5 \times 10^{-5}\right.$ and $\left.2.51 \leq H_{12} \leq 2.6\right)$ the resulting functions show similar trends. It was found that the transition Reynolds number decreases with increasing $p^{\star}$ (Fig. 5.19) and also with increasing $H_{12}$ (Fig. 5.18), as expected (Schlichting and Gersten, 2000).

Since the different freestream disturbance levels are corrected in Figs. 5.18 and 5.19, the transition Reynolds numbers at different Mach numbers can be compared conclusively. It can be seen that the transition Reynolds number increases significantly with Mach number. This result reveals the expected trend of larger Mach numbers leading to larger transition Reynolds numbers at the same freestream disturbance level, the reason being the stabilizing effect of compressibility on T-S waves (Arnal and Vermeersch, 2011).

\subsubsection{Calculation and correction of the critical $\mathrm{N}$-factor}

The $N$-factor method is a widely used method for transition prediction on two-dimensional boundary layers (van Ingen, 2008). However, large discrepancies have been found in the determined critical $N$-factors (Schrauf, 1994, 2000, 2005, Schrauf et al., 1996, 1998). In the first part of this section, the results of compressible and incompressible critical $N$-factors are compared and the influences of unit Reynolds number, Mach number and shape factor on $R e_{t r}^{\star}$ are discussed (Sects. 5.7.2.1 and 5.7.2.2). In the second part, two approaches for the correction of determined critical $N$-factors, based on the spectral level of total pressure fluctuations (Sect. 5.7.3.1), and the incidence angle dependent receptivity of acoustic disturbances (Sect. 5.7.3.2) are summarized and discussed.

\subsubsection{Mach number influence on compressible and incompressible critical $N$-factors}

Local linear stability analysis was used to determine critical $N$-factors with compressible and incompressible theory for all available data points. An average compressible critical $N$-factor of $N_{\text {comp }}=8.50$, with a standard deviation of $\sigma_{\text {comp }}=1.14$, and an average incompressible critical $N$-factor of $N_{i n c}=8.99$, with a standard deviation of $\sigma_{i n c}=0.95$, were found for PaLASTra in DNWKRG. When all data points are compared, the maximal variations $\left(\Delta N=N_{\max }-N_{\min }\right)$ show the same trends as the standard deviations: the determined compressible critical $N$-factor exhibit larger variations $\left(\Delta N_{\text {comp }}=4.90\right)$ as compared to the incompressible critical $N$-factor $\left(\Delta N_{\text {comp }}=4.29\right)$. The same trends have been found by Schrauf (1994, 2000) and Schrauf et al. (1998), who report that critical $N$-factors calculated with incompressible theory exhibit a better correlation than critical $N$-factors calculated with compressible theory. The larger fluctuations of compressible critical $N$-factors are due to their Mach number dependence, as shown in Fig. 5.23 the compressible critical $\mathrm{N}$-factors decrease with increasing Mach number, which agrees with findings by Schrauf (2000).

At first sight, the observed decrease of compressible critical $N$-factors with $M$ appears to be contradictory to what one would expect physically: as the compressible $N$-factor analysis also 
captures the stabilizing effect of compressibility on the boundary layer, a smaller variation with Mach number would be expected (Arnal and Vermeersch, 2011; Schrauf et al., 1996). However, it is very likely that the stabilizing effect of compressibility is compensated by a destabilizing effect of increasing flow disturbances with Mach number (Arnal, 1989), which may explain the better correlation of the determined incompressible critical $N$-factors. Therefore, the influence of pressure fluctuations on the determined critical $N$-factor is discussed in more detail below (Sect. 5.7.3).

It is also interesting to note that, if critical $N$-factors of different Mach numbers are compared separately, as done in Sect. 5.5. compressible critical $N$-factors exhibit a smaller standard deviation and a smaller maximal variation than the incompressible critical $N$-factors (Table 5.7). This observation is in line with the Mach number dependence as discussed above. Furthermore, it shows that, it is not valid to assume, in general, a better correlation of the incompressible critical $N$-factor as compared to the compressible critical $N$-factor. Instead, the influence of the external disturbance spectrum has to be considered and corrected. However, in case the influence of the external disturbance spectrum on the critical $N$-factor is not corrected, actually incompressible critical $N$-factors were found to show a better correlation.

\subsubsection{Influence of unit Reynolds number on critical $N$-factors}

In a wind tunnel test, as reported by Schrauf (2000), it has been found that compressible and incompressible critical $N$-factors depend on unit Reynolds number. However, the trends Schrauf (2000) found in the wind tunnel, contradict the trends that were found in flight tests. This observation was explained by an increasing disturbance amplitude of the wind tunnel tests with unit Reynolds number (Schrauf, 2000). In the current investigation a slight increase (with a maximum of $\Delta N \approx 1$ ) of the critical $N$-factor with unit Reynolds number at small $H_{12}$ was found (see also Figs. A.5 to A.10 in Appendix). However, when all data points were compared (Figs. 5.20 and 5.21 ) the trend was not found to be stringent and lies within the measurement accuracy, as discussed below (see Sect. 5.6.3). This observation can be explained by the fact that the disturbance amplitude of pressure fluctuations in DNW-KRG is independent of unit Reynolds number in the relevant frequency range (see Sect. 5.4.1.

\subsubsection{Correction of the determined critical $N$-factors}

In Sect. 5.5 two steps were made to correct the influence of pressure fluctuations on the determined critical $N$-factors. The first step, which was described in Sect. 5.5.1, aims to correct the critical $N$-factor values taking into account the Mach number dependence of the amplitude of freestream total pressure fluctuations whose frequency corresponds to the T-S wave responsible for transition and will be discussed in Sect.5.7.3.1. The second step, which was described in Sect.5.5.2, focuses on the receptivity dependency of acoustic disturbances, which varies with incidence angle and will be discussed in Sect. 5.7.3.2. 


\subsubsection{Correction of total pressure fluctuations}

As explained above, it is likely that the found Mach number dependency of the compressible critical $\mathrm{N}$-factors is caused by an increasing spectral level of total pressure fluctuations with Mach number (analyzed in Sect. 5.4.1). Furthermore, the frequency dependency of the spectral level of total pressure fluctuations is likely to be at least partially responsible for the described influence of unit Reynolds number and shape factor on the determined critical $N$-factors.

To correct the influence of an increasing level of total pressure fluctuations with Mach number, the compressible and incompressible critical $N$-factors were corrected by relating the spectral level of total pressure fluctuations to the initial amplitude of the T-S waves linearly (Sect. 5.5.1). By this correction, the standard deviations and maximal variations were reduced for the incompressible $\left(N_{\text {inc }, p^{\star}}=9.60 \pm 0.84(4.06)\right)$ and compressible critical $N$-factors $\left(N_{\text {comp }, p^{\star}}=9.11 \pm 0.87(4.02){ }^{15}\right.$ The improvement in $\sigma$ and $\Delta N$ is significantly larger in the compressible case (an average improvement of about $21 \%$ ) than in the incompressible case (an average improvement of about $8 \%$ ), when all data points are compared. By comparing the $p^{\star}$-corrected critical $N$-factors, shown in the middle of Figs. 5.20 and 5.21, it can be seen that the variation is significantly reduced, compared to $N_{i n c}$ and $N_{\text {comp }}$, shown at the top of Figs. 5.20 and 5.21 .

Furthermore, it should be noted that the correction of the incompressible critical $N$-factor $\left(N_{i n c, p^{\star}}\right)$ produces new outliers. This observation is also resembled by the (slightly) smaller maximal variation of the compressible case $\left(\Delta N_{c o m p, p^{\star}}=4.02\right)$ compared to the incompressible case $\left(\Delta N_{i n c, p^{\star}}=4.06\right)$, which is even more significant when Mach numbers are compared individually (Table 5.7). Therefore, it can be conjectured that the $p^{\star}$-corrected compressible critical $N$-factors $\left(N_{c o m p, p^{\star}}\right)$ capture more of the relevant physical processes leading to transition. In contrast, it can also be conjectured that the incompressible critical $N$-factors capture in general less physical processes and are, therefore, less suitable for transition prediction when the spectral level of total pressure fluctuations are incorporated.

\subsubsection{Correction of the dependence on incidence angle of receptivity of acoustic disturbances}

Apart from total pressure fluctuations, also acoustic disturbances have a significant influence on T-S induced transition, as discussed in Sect. 5.5.2. Since receptivity of acoustic disturbances depends strongly on the incidence angle (which was assumed to correspond to the angle-of-attack), a linear approximation based on calculations of Heinrich et al. (1988) was used to correct the determined critical $N$-factors.

It was found that the determined critical $N$-factors, $N_{p^{\star}, C}$, with corrected influence of total pressure fluctuations $\left(p^{\star}\right)$ and receptivity of acoustic disturbances $(C)$, exhibit the smallest standard deviations $(\sigma)$ and the smallest maximal variations $(\Delta N)$ of all cases. The values of $\Delta N$ and $\sigma$ are smaller for $N_{c o m p, p^{\star}, C}$ compared to $N_{i n c, p^{\star}, C}$ (except for a slightly smaller standard deviation of $N_{\text {inc }, p^{\star}, C}$, when all data points are compared). It can therefore be conjectured that the $C$ - and $p^{\star}$ -

\footnotetext{
${ }^{15}$ Notation: The critical $N$-factor $(N)$, its standard deviation $(\sigma)$ and maximal variation $\left(\Delta N=N_{\max }-N_{\min }\right)$ are summarized here, as in Table 5.7 with: $N \pm \sigma(\Delta N)$.
} 
corrected compressible critical $N$-factors $\left(N_{c o m p, p^{\star}, C}\right)$ capture the most relevant physical processes in this study.

Furthermore, it is interesting to note that the dependence of the $C$-corrected critical $N$-factors ( $N_{p^{\star}, C}$ ) on $H_{12}$ could be reduced by the employed corrections, but it could not be removed completely. A better cancellation would be possible if a significantly larger dependence of receptivity on the angle-of-attack would be assumed. The slope $c$ in Eq. 5.11 would be required to be about twenty times larger, which appears to be too large to be reasonable at first sight. However, the assumption that the angle-of-attack is identical with the angle of incidence (assumption 3 of Sect. 5.5.2) might not be fully valid, since acoustic waves may not be aligned with the flow direction. Furthermore, the receptivity process is still not completely understood and the receptivity coefficients determined in past work have been found to vary by an order of magnitude (Heinrich et al., 1988; Shahriari et al. 2016). Additionally, it must be considered that the freestream disturbances in the experiment might not enter the boundary layer only at the leading edge, but also at discontinuities of the model surface, such as model part junctions (see also Sect.5.3.2. Also non-parallel effects, non-linear mechanisms and influences of nose bluntness would need to be considered (Arnal et al., 1997). The knowledge of this missing information would probably further improve the prediction of transition.

In this context it should be emphasized that the concept of a universal critical $N$-factor is based on the assumption of linear growth of Tollmien-Schlichting waves. However, the transition from laminar to turbulent flow is a non-linear process (Würz et al. 2012a b). Therefore, the existence of a universal $N$-factor, which is completely independent of $M, \operatorname{Re}_{1}$ and $\alpha$ cannot be expected.

\subsection{Conclusion}

The described improvements in the spatial and temporal transition detection method of TSP and the quantitative surface temperature measurement allowed the determination of the transition Reynolds number with a high accuracy. Since the measured surface temperature distribution was also incorporated into boundary layer calculations, it was also possible to account for temperature effects on the $\mathrm{N}$-factor calculation. These advances in the measurement technique allowed the systematic investigation of unit Reynolds number, Mach number and pressure gradient effects on laminar-turbulent transition in two-dimensional boundary layers.

The modified PaLASTra model has shown its capability to study laminar-turbulent transition in two-dimensional flows systematically for various Mach numbers, unit Reynolds numbers and pressure gradients in DNW-KRG. The modifications of the PaLASTra model proved to be useful to reduce the magnitude of pressure fluctuations caused by flow separation at the trailing edge of the original model below the minimum observable quantity. It was found that the model exhibits a quasi-uniform streamwise pressure gradient, characterized by the incompressible shape factor, $H_{12}$. A linear dependence between $H_{12}$ and the transition Reynolds number was found for each combination of Mach and unit Reynolds number, which was approximated by linear functions with the intercepts, $h_{\mathrm{II}, 0}$, and slopes, $h_{\mathrm{II}, 1}$.

It was therefore possible to show in this study that not the RMS turbulence level alone is meaningful for T-S induced transition, but instead the spectral level in the relevant frequency range 
has to be considered, as conjectured by Meier et al. (1987). The relation between the spectral level and the frequency range relevant for T-S induced transition was used to explain and quantify the observed unit Reynolds number effect on the transition Reynolds number. To this end, an analytic function was derived which approximates the transition Reynolds number as a function of unit Reynolds number. A power law relation between unit Reynolds number and transition Reynolds number was used, with $R e_{t r} \sim R e_{1}^{\alpha_{\text {III }}}$. The exponent $\alpha_{\text {III }}$ was found to range between 0.1 and 0.6 for accelerated flows, which agrees well with measurements in hypersonic wind tunnels (Arnal, 1989).

The unique feature of DNW-KRG to vary Mach number by adjusting the cross section of the sonic throat downstream of the test section and unit Reynolds number by adjusting the pressure of the working gas (Rosemann, 1997), was also used to conduct a systematic study of the Mach number effect (or 'compressibility effect') on the location of laminar-turbulent transition. Therefore, the systematic variation of $M, R e_{1}^{\star}$ and $H_{12}$ was combined with a detailed analysis of the freestream turbulence spectrum in the wind tunnel, which allowed the experimental quantification of the Mach number effect. Hence, the presented analysis gives the first systematic and quantitative description of the unit Reynolds number and Mach number effect in a subsonic flow.

A compressible and incompressible linear stability analysis was carried out and the critical $\mathrm{N}$-factors were determined. It was shown, in agreement with earlier findings (Schrauf, 1994. 2000, 2005; Schrauf et al. 1996, 1998), that the exclusive incorporation of compressibility into linear stability analysis leads to a larger deviation in the determined critical $N$-factors as compared to incompressible stability analysis. However, when not only compressibility but also the varying influence of the spectral level of total pressure fluctuations on the initial T-S wave amplitude is incorporated in the $N$-factor analysis, the correlation of compressible critical $N$-factors is equally good or even better than in the incompressible case.

The approach to develop a correction of the $e^{N}$-method is in line with earlier attempts of other researchers (e.g. Mack (1977); van Ingen (2008)), who used the RMS turbulence level to correct the critical $N$-factor. However, the correction presented here is not only based on the RMS turbulence level, but on the level of external disturbances in the frequency range relevant for T-S waves leading to transition. In contrast to earlier approaches (Mack, 1977, van Ingen, 2008), the presented correction method leads to good results in the investigated low-turbulence environment.

The determined critical $N$-factors were also found to show a dependency on $H_{12}$, which agrees with findings in Arnal et al. (1997). These effects have been accounted to shortcomings of the $e^{N}$ method as, for instance, nonparallel effects, the receptivity process or non-linear mechanisms (Arnal et al. 1997). As an attempt to correct the influences of receptivity process, a correction method was developed in this study: the receptivity dependency of acoustic disturbances on incidence angles was incorporated in the correction of the determined critical $N$-factors. It was found that, even though the chosen parameters (based on investigations by Heinrich et al. (1988)) were not large enough to remove the complete dependency of the determined critical $N$-factors on $H_{12}$, this dependency could be reduced. The correlation of the determined critical $N$-factors was thus further improved when the receptivity of acoustic disturbances was also corrected by the suggested method.

The average critical $N$-factors of $N_{c o m p, p^{\star}, C}=9.36$ with a standard deviation of $\sigma_{c o m p, p^{\star}, C}=0.78$ and $N_{i n c, p^{\star}, C}=9.85$ with $\sigma_{i n c, p^{\star}, C}=0.76$ proved that the modified PaLASTra model in DNW-KRG 
provides a well suited experimental setup for laminar flow investigations. It can be concluded that the modified PaLASTra model can be a useful tool to study laminar-turbulent transition in two-dimensional flows systematically.

Acknowledgements We would like to thank A. Dillmann, L. Koop, M. Rein, H. Rosemann (all DLR) for their support and advise; U. Becker (DLR), C. Fuchs (DLR), T. Kleindienst (DLR), V. Ondrus (Univ. Hohenheim) and L. Schojda (DLR) for model and test preparation; U. Henne, M. Hilfer, J. Ost and A. Weiss (all DLR) for their help during the measurement campaign, data evaluation and interpretation. Furthermore we would like to thank M. Aschoff, S. Hucke, R. Kahle and K. Plettenberg (German-Dutch Wind Tunnels) for the support during the wind-tunnel test campaigns. We also thank the anonymous reviewers for their helpful comments and corrections which further improved the manuscript. 



\title{
Part III
}

\section{CONCLUSION}

\author{
6 Summary and conclusion \\ 7) Closing remarks and outlook
}





\section{Summary and conclusion}

In the accumulated published papers of this thesis, the results from applying a new quantitative and time-resolved surface temperature measurement technique are presented. The results include the determination of heat transfer in a hypersonic flow, improvements in the detection of transition locations and analyses of the influences of free stream disturbances, Mach number, Reynolds number and pressure gradient effects on the transition Reynolds number. Furthermore, software tools for boundary-layer calculations (COCO) and linear stability analysis of Tollmien-Schlichting waves $(L I L O)$ were extended to incorporate the effects of surface temperature distributions and two methods were developed to correct the influence of free stream disturbances on the determined critical $N$-factors. To summarize the accomplishments, the ten questions formulated in the motivation section (Chapter 1) are addressed with respect to the detailed results described in the corresponding chapters.

1. How can the temperature on a model surface be measured non-intrusively, time-resolved and quantitatively?

In order to conduct a time-resolved quantitative measurement of the surface temperature on a model surface in a shock tunnel, a temperature-sensitive paint was employed which was calibrated in an external calibration device (Chapter 3 and Appendix B). The calibration device allows the systematic variation of temperature and pressure as well as the simultaneous measurement of the luminescent intensity of the TSP, which is excited with light of an appropriate wave length (Egami et al. 2012). By this calibration procedure a function can be obtained which relates the normalized intensity to the temperature measured by TSP (Eq. B.4). The calibration function is used to calculate the unknown temperature distribution on a surface from a measured distribution of the luminescent intensity (Fig. 3.4). The TSP used in the current study exhibits almost no dependence on pressure but a high temperature sensitivity of about $-3.7 \% \mathrm{~K}^{-1}$ to $-5 \% \mathrm{~K}^{-1}$ (Ondrus et al. 2015).

The surface temperature measurement described in Chapter 3 was carried out in the High Enthalpy Shock Tunnel Göttingen (HEG), which is a short-duration test facility that allows the measurement at supersonic and hypersonic flow speeds (Hannemann and Martinez Schramm, 2007). Since the HEG is an impulse facility the total test time was less than $10 \mathrm{~ms}$ (HEG test condition XIV). Within this test time 50 images with an exposure time of $200 \mu$ s were obtained, which corresponds to a sampling rate of $5 \mathrm{kHz}$. Therefore, it was necessary to use a high speed camera and a high power LED to achieve a sufficient illumination of the test sample (Sec. 3.3).

The quantitative temperature distributions, obtained with the time-resolved measurement system are presented in Chapter 3 . The measured temperature distribution is shown in Fig. 3.9 and compared to the reading of a Medtherm sensor in Fig. 3.10 and Fig. 3.17. Because the lifetime of the TSP is within the time range of the exposure time, an elaborate discussion of measurement 
uncertainties was also conducted (Sec. 3.6.4.3).

\section{How can heat transfer be determined from the time-resolved temperature information?}

In order to calculate the heat transfer from the obtained time-resolved temperature information a one-dimensional heat transfer model was assumed having a semi-infinite depth ( $\mathrm{Sec}$. 3.5). It has been shown that these assumptions are valid for typical test times in shock tunnels (Martinez Schramm et al. 2015). Based on these assumptions, a simple relationship can be derived between the temperature and heat transfer (Cook and Felderman, 1966; Hannemann and Martinez Schramm, 2007; Schultz and Jones, 1973). However, apart from the temperature information, the model requires the determination of the thermal parameter $\sqrt{\rho c k}$ of the TSP. The thermal parameter was determined by an insitu calibration as well as by a separate thermal analysis of the TSP (Sec. 3.6.4.1). It is also shown in Chapter 3 that the values obtained with both approaches are in the same range, lending further weight to their trustworthiness. The resulting heat transfer distribution on the surface of the test model is determined with TSP and calculated using the insitu calibration (Figs. 3.11 and 3.12).

3. How can locations of laminar-turbulent transition be reliably determined from surface temperature (or TSP intensity) distributions?

In order to detect laminar-turbulent transition of a boundary layer on a model surface it is possible to make use of the different heat transfer coefficients between laminar and turbulent boundarylayers. Due to the large mixing of turbulent flows, convective heat transfer is larger in a turbulent than in a laminar boundary-layer. If the temperature of the external flow differs from the model surface temperature, the surface temperature of the model adjusts faster to the surrounding flow temperature in the turbulent region than in the laminar region. This temperature difference can be visualized by temperature-sensitive paint which is applied on the surface of the model (Fey and Egami, 2007). The transition location can be defined by finding the maximum temperature gradient in flow direction (Risius, 2016). An algorithm which incorporates the detection of the maximum temperature gradient on the three-dimensional grid of the test model was integrated in the nToPas software, which was used for the evaluation of TSP images (Appendix B and Costantini et al. (2018)).

In a first step the described method for transition detection was carried out at only a few specific spanwise locations. At these locations a chordwise cut in flow direction was evaluated in order to determine the position of laminar-turbulent transition (Fig. 4.4). In a second step, the transition detection algorithm was extended over the complete span, as shown in Fig.5.3. Both approaches are compared in Fig. B.16 of the Appendix B. The extended method allows both, a more reliable approach for transition detection, as well as a quantification of the variations of transition locations in spanwise directions.

4. How can the influences of surface temperature distributions on the transition Reynolds number of two-dimensional flows be quantified and used as a correction?

As described in the discussion of the previous question, a temperature difference between the flow and the model is to see transition detection with TSP. In the Cryogenic Ludwieg-Tube (DNW$\mathrm{KRG}$ ) the required temperature step is induced by expansion of the working fluid which leaves the 
storage tube during the test run and expands through the test section (Koch, 2004). The temperature difference between the flow and the model also influences the stability of the boundary layer and, therefore, the location of laminar-turbulent transition (Schlichting and Gersten, 2000).

In earlier measurements the influence of the temperature difference between flow and model on the transition location was measured (Costantini, 2016). The obtained dependence was approximated linearly in Chapters 4 and 5 to correct the influence of the model temperature on the measured transition Reynolds numbers (Eqs. 4.1 and 5.4). Therefore, it was possible to correct for the influences of non-adiabatic model surface temperatures on the measured transition Reynolds numbers.

5. What is the effect of pressure gradient variations on the transition Reynolds number and how can it be quantified?

As expected, it was found that an increased acceleration of the boundary layer due to a favourable pressure gradient leads to an increased transition Reynolds number, (Schlichting and Gersten, 2000). For the PaLASTra model a linear dependence of the transition Reynolds number on the Hartree parameter (Fig. 4.6) and shape factor (Figs. 5.6 and 5.10) was found. For a further analysis the transition Reynolds number was approximated as a linear function of the incompressible shape factor for three different Mach numbers and six different unit Reynolds numbers (Fig. A.1 and Tables 5.2 and 5.3. The determined coefficients of the approximations (slope and intercept) were used to study the effects of unit Reynolds number and Mach number in more detail, which will be described in the following.

\section{How can the unit Reynolds number effect on transition Reynolds numbers be quantified?}

It is known that in wind tunnel measurements at hypersonic flow speeds the transition Reynolds number increases with the unit Reynolds number, which is referred to as 'unit Reynolds number effect' (Sec. 5.2.1). The unit Reynolds number effect is caused by an increasing frequency of Tollmien-Schlichting waves with increasing unit Reynolds number and the simultaneous decrease of energy of free flow disturbances with increasing frequency. The described trends were investigated and quantified for the first time in a transonic wind tunnel in Chapter 5 .

Based on linear approximations of the transition Reynolds number as a function of the shape factor (which was used to characterize the pressure gradient of the boundary layer) it was possible to investigate the unit Reynolds number effect in more detail (Sec. 5.7.1.1). The dependence of transition Reynolds number on the unit Reynolds number was given by a power law relation (Eq. III and Fig. 5.11). The approximated coefficients used for the power law relation were determined by quadratic approximations (Fig. 5.13). The approximative relation makes it possible to calculate the transition Reynolds number of the PaLASTra model for any given value of shape factor and unit Reynolds number (Figs. 5.13 and 5.14). It was found that the transition Reynolds number increases with $R e_{1}^{\star}$ and decreases with $H_{12}$, as expected.

7. What are the effects of free stream disturbances on boundary-layer transition and how can they be quantified and corrected?

The quantification of free stream turbulence parameters is an important aspect in many different fields of fluid dynamics. It plays an important role not only in the formation of clouds and in 
the quantification of atmospheric flows (Risius et al., 2015b), but also has a strong impact on the location of laminar-turbulent transition in a boundary layer (Chapters 4 and 5). Although the practical aspect of these two examples is quite different, both fields of science can learn much from earth other, as the detailed analysis of turbulence spectra shows. In Chapter 5 the power spectrum of total pressure fluctuations measured in DNW-KRG (Fig. 5.9p was used to quantify and correct the influence of free stream disturbances on transition Reynolds numbers (Figs. 5.18 and 5.19) and critical $N$-factors (Figs. 5.20 and 5.21). Therefore, the intensity of total pressure fluctuations in the frequency range relevant for Tollmien-Schlichting-induced transition was quantified and the transition Reynolds numbers (Eq. 5.7) and critical $N$-factors (Eq. 5.10) were normalized according to values obtained at a reference level of free stream disturbances. As a result, it was possible to compare transition Reynolds numbers (Sec. 5.4.6.2) and critical $N$-factors (Sec. 5.5.1) which were obtained at different levels of free stream total pressure fluctuations.

This correction of free stream disturbances is important to wind tunnel measurements since the spectrum of the disturbances changes depending on the flow conditions, e.g. Mach number, Reynolds number (Sec. 5.2). Also the frequency range which is most relevant for TollmienSchlichting-induced transition depends on the flow conditions (Sec. 5.4.5). It may therefore not be meaningful to compare transition Reynolds numbers and critical $N$-factors which were obtained at different Mach and Reynolds numbers or angles-of-attack directly (Secs. 4.4.2 and 5.4.4). Instead, a correction of the transition Reynolds numbers or critical $N$-factors is necessary, in order to allow the comparison of different values (Eqs. 5.7 and 5.10). This observation becomes even more important when one considers that the level of free stream disturbances in free flight is generally much lower than that achievable in a wind tunnel (Fisher and Dougherty, 1982). In order to allow a transfer of transition measurements to free flight conditions it is essential to conduct corrections of the measurements, such as the ones presented in Chapter 5 . After the described correction methods have been applied to the obtained transition data, it is meaningful to investigate the effects of Mach number, Reynolds number and pressure gradient on two-dimensional boundary-layer transition, as described in Secs. 5.7.1.1, 5.7.1.2 and 5.7.3.1.

8. What is the effect of compressibility on the transition Reynolds number and how can it be quantified?

The stabilizing effect of compressibility due to an increasing Mach number as predicted by linear stability theory (Arnal and Vermeersch, 2011) was systematically investigated in Chapters 4 and 5 under subsonic flow conditions. The correction methods described above made it feasible to compare transition Reynolds numbers obtained at different Mach numbers and, each at different levels of turbulence intensity (Secs. 4.4 and 5.4.6.2). The correction was done by finding the relevant frequency range of Tollmien-Schlichting waves leading to transition and normalizing the transition Reynolds number using a reference level of total pressure fluctuations (Eq. 5.7). The discovered trends of the Mach number influences show a significant indication of the expected stabilization by compressibility (Figs. 5.18 and 5.19).

9. How can transition prediction based on linear stability theory and analysis of the free stream disturbance spectrum be improved? 
The $e^{N}$-method is a standard method for transition prediction on airfoils. It is based on the assumption that transition takes place where the most unstable frequency of a Tollmien-Schlichting wave is amplified by a factor of $e^{N}$, where $N$ is a universal constant, which is referred to as critical $N$-factor. However, it has been found that the critical $N$-factor is not a universal constant but depends on the wind tunnel and free flow disturbances (van Ingen, 2008). Therefore, the critical $\mathrm{N}$-factor is usually determined by correlating a measured transition location with amplification rates of Tollmien-Schlichting waves (Fig. 4.5). In order to correct the influence of free flow disturbances a first correction method based on the RMS turbulence level has been proposed by Mack (1977). In Chapter 5 a new correction method is proposed that is not only based on the RMS turbulence level but on the energy of total pressure fluctuations in the frequency range of Tollmien-Schlichting waves leading to transition (Sec. 5.5.1). The initial amplitude of the Tollmien-Schlichting wave is normalized to a reference value depending on the energy of the free flow total pressure fluctuations (Sec. 5.7.3.1).

However, total pressure fluctuations quantify only one possible aspect of free flow disturbances. Acoustic disturbances have also an important influence on Tollmien-Schlichting induced transition. Because the receptivity of the boundary layer to acoustic noise depends strongly on incidence angles, a further correction method was developed (Sec. 5.5.2). It corrects the critical $N$-factors depending on the incidence angles of acoustic waves which are assumed to depend on the anglesof-attack. As reported in Chapter 5 it was found that a significant improvement in the correlation of the determined critical $N$-factors of the PaLASTra model is achieved by these correction methods (Table 5.7 and Figs. 5.20, 5.21 and 5.7).

Another important step to improve the transition prediction with linear stability analysis was to incorporate the chordwise temperature distribution, measured with temperature-sensitive paint, into the boundary-layer solver $C O C O$ (Secs. 4.3 and 5.3.3). The calculated boundary-layer profile was used by the linear stability analysis tool $L I L O$ in order to calculate the amplification rates of Tollmien-Schlichting waves 5.3.4. Due to incorporation of the measured temperature distribution the calculated amplification rates are shown to be more meaningful (Figs 4.5 and 5.8). The first results obtained with this procedure are presented in Chapter 4.

10. Which influences do compressible and incompressible linear stability analysis have on the correlation of the critical $N$-factors?

Linear stability theory can be conducted, using either incompressible or compressible linear stability equations (Secs. 4.4.2 and 5.2.4). It has been found in earlier measurements that determined critical $N$-factors show a better correlation when incompressible, rather than compressible linear stability analysis is used (Schrauf, 2000; Schrauf et al., 1998). The same trends have been found in this study and are reported in Chapters 4 and 5 .

However, before this study, it had not been understood why incompressible stability analysis leads to a better correlation of the determined critical $N$-factors (Sec. 4.4.2). This observation contradicts the general expectation: a model which incorporates more physical processes (i.e. here compressibility effects) nevertheless produces less consistent results (Sec. 5.2.4). After the correction of free stream disturbances of the determined critical $N$-factors, a better correlation of 
the compressible critical $N$-factors was observed (Chapter 5.5.1). From the results of the correction methods it can be concluded that the influences of total pressure fluctuations are most likely the cause for the observed behaviour (Sec. 5.7.3.1). 


\section{Closing remarks and outlook}

It was shown that the described measurement of time-resolved quantitative surface temperature distributions have led to many important results which may have both practical and fundamental (or philosophical) aspects, as Reynolds (1884) put it. The described work will be continued in the following two directions which should augment each other.

Future fundamental research is directed towards the measurement of fluid dynamic quantities by the temperature-sensitive paint method. For example, the measurement of heat transfer can give important information for the calculation of wall-shear stresses via the Reynolds analogy. Especially promising concerning a quantification of wall-shear stress measurements with temperature-sensitive paint are experiments in water, as reported by Lemarechal et al. (2018).

The more practical direction of active research is the investigation of the influence of twodimensional surface imperfections on boundary-layer transition. In order to adopt Natural Laminar Flow airfoils to commercial flight, the determination of manufacturing tolerances is crucial. Twodimensional imperfections, such as steps and gaps, will be investigated in the Cryogenic LudwiegTube in order to quantify their influence on laminar-turbulent transition. This work leads in the direction described by Costantini et al. (2015b). 



\section{Bibliography}

Contribution of working group I to the fourth assessment report of the intergovernmental panel on climate change. 2007. URL/http://www.ipcc.ch/publications_and_data/ar4/wg1/en/ contents.html.

Engineering ToolBox, 2015. URL www . engineeringtoolbox.com.

ACARE. Strategic Research and Innovation Agenda - 2017 update, 2017. URL http://www.acare4europe.org/documents/ strategic-research-innovation-agenda-2017-update.

D. Arnal. Laminar-turbulent transition problems in supersonic and hypersonic flows. In AGARD Special Course on Aerothermodynamics of Hypersonic Vehicles, number AGARD-R-761, pages 8-1-8-45. Rhode-Saint-Genèse, Belgium, 1989.

D. Arnal. Boundary layer transition: Prediction, application to drag reduction. Technical Report AGARD Rept. 786, 1992.

D. Arnal and J. Délery. Laminar-turbulent transition and shock wave/boundary layer interaction. In RTO AVT Lecture Series on Critical Technologies for Hypersonic Vehicle Development, RTO-EN-AVT-116, pages 4-1-4-46. Rhode-St-Genèse, Belgium, 2004.

D. Arnal and O. Vermeersch. Compressibility effects on laminar-turbulent boundary layer transition. International Journal of Engineering Systems Modelling and Simulation, 3(1-2):26-35, Jan. 2011. doi:10.1504/IJESMS.2011.038747.

D. Arnal, G. Casalis, J. Cousteix, and J. Reneaux. Laminar-turbulent transition in subsonic boundary layers - Research and applications in France. In 28th Fluid Dynamics Conference. American Institute of Aeronautics and Astronautics, June 1997. doi:10.2514/6.1997-1905.

K. Asai, W. Beck, M. Costantini, Y. Egami, R. H. Engler, U. Henne, C. Klein, L. Koop, Y. Le Sant, Y. Mebarki, M.-C. Merienne, V. Ondrus, J. Ost, A. Weiss, D. Yorita, I. Micknaus, C. Rosenstock, J. Agocs, C. Fuchs, T. Kleindienst, and S. Risius. Application of Pressure/Temperature Sensitive Paint Theory and Practice. Göttingen, Deutschland, 2017. URL http://elib.dlr.de/ 117566/.

W. H. Beck, C. Klein, U. Henne, J. M. Schramm, A. Wagner, K. Hannemann, T. Gawehn, and A. Guelhan. Application of temperature and pressure sensitive paints to DLR hypersonic facilities: "lessons learned". In 53rd AIAA Aerospace Sciences Meeting, volume 2015-0023. American Institute of Aeronautics and Astronautics, 2015. doi:10.2514/6.2015-0023 
P. Blasius. Grenzschichten in Flüssigkeiten mit kleiner Reibung. Zeitschrift fur Mathematik und Physik, 56(1), 1908.

L. I. Boehman and M. G. Mariscalco. Stability of highly-cooled compressible laminar boundary layers. Technical Report UDRI-TR-76-70, Dayton University, Ohio, USA, Oct. 1976.

B. H. Carmichael. Surface waviness criteria for swept and unswept laminar suction wings. Technical Report NOR-59-438 (BLC-123), Northrop Aircraft Inc., 1959.

CleanSky. Joint Technology Initiative. URL http://www.cleansky.eu/

W. J. Cook and E. J. Felderman. Reduction of data from thin-film heat-transfer gages - A concise numerical technique. AIAA Journal, 4(3):561-562, 1966. doi:10.2514/3.3486.

M. Costantini. Experimental Analysis of Geometric, Pressure Gradient and Surface Temperature Effects on Boundary-Layer Transition in Compressible High Reynolds Number Flow. PhD Thesis, RWTH Aachen University, Aachen, 2016. URL http://elib.dlr.de/117965/.

M. Costantini, U. Fey, U. Henne, and C. Klein. Influence of non-adiabatic model surface on transition measurements using the temperature-sensitive paint technique in a cryogenic wind tunnel. American Institute of Aeronautics and Astronautics, June 2012. doi:10.2514/6.20122830.

M. Costantini, U. Fey, U. Henne, and C. Klein. Nonadiabatic surface effects on transition measurements using temperature-sensitive paints. AIAA Journal, 53(5):1172-1187, May 2015a. doi:10.2514/1.J053155.

M. Costantini, S. Risius, and C. Klein. Experimental investigation of the effect of forward-facing steps on boundary layer transition. Procedia IUTAM, 14:152-162, $2015 \mathrm{~b}$. doi:10.1016/j.piutam.2015.03.036.

M. Costantini, S. Hein, U. Henne, C. Klein, S. Koch, L. Schojda, V. Ondrus, and W. Schröder. Pressure gradient and nonadiabatic surface effects on boundary layer transition. AIAA Journal, 54(11):3465-3480, Nov. 2016a. doi:10.2514/1.J054583.

M. Costantini, S. Risius, C. Klein, and W. Kühn. Effect of forward-facing steps on boundary layer transition at a subsonic Mach number. In New Results in Numerical and Experimental Fluid Mechanics X, Notes on Numerical Fluid Mechanics and Multidisciplinary Design, pages 203-213. Springer, Cham, 2016b. doi:10.1007/978-3-319-27279-5_18.

M. Costantini, U. Henne, S. Risius, and C. Klein. A robust method for reliable transition detection. 2018. submitted.

J. Crouch. Boundary-layer transition prediction for laminar flow control. American Institute of Aeronautics and Astronautics, June 2015. doi $10.2514 / 6.2015-2472$.

P. Czysz and W. P. Dixon. Quantitative heat transfer measurement using thermographic phosphors. Optical Engineering, 7(3):070377, Mar. 1969. doi:10.1117/12.7971513. 
P. A. Davidson. Turbulence: An Introduction for Scientists and Engineers. Oxford University Press, Oxford, UK ; New York, 1 edition edition, July 2004.

L. de Luca, G. M. Carlomagno, and G. Buresti. Boundary layer diagnostics by means of an infrared scanning radiometer. Experiments in Fluids, 9(3):121-128, May 1990. doi:10.1007/BF00187411.

Y. Egami, U. Fey, and J. Quest. Development of New Two-Component TSP for Cryogenic Testing. In AIAA Aerospace Sciences Meeting and Exhibit, pages 8-11, 2007.

Y. Egami, U. Fey, C. Klein, J. Quest, V. Ondrus, and U. Beifuss. Development of new twocomponent temperature-sensitive paint (TSP) for cryogenic testing. Measurement Science and Technology, 23(11):115301, 2012. doi:10.1088/0957-0233/23/11/115301

R. H. Engler, K. Hartmann, and B. Schulze. Aerodynamic assessment of an optical pressure measurement system (OPMS) by comparison with conventional pressure measurements in a high speed wind tunnel. In International Congress on Instrumentation in Aerospace Simulation Facilities, pages 17-24, 1991. doi:10.1109/ICIASF.1991.186220.

R. H. Engler, C. Klein, and O. Trinks. Pressure sensitive paint systems for pressure distribution measurements in wind tunnels and turbomachines. Measurement Science and Technology, 11(7): 1077, 2000. doi:10.1088/0957-0233/11/7/320

E. Erturk and T. C. Corke. Boundary layer leading-edge receptivity to sound at incidence angles. Journal of Fluid Mechanics, 444:383-407, Oct. 2001. doi 10.1017/S0022112001005456.

A. Fage. The smallest size of a spanwise surface corrugation which affects boundary layer transition on an airfoil. ARC Reports and Memoranda 2120, 1943.

V. M. Falkner and S. W. Skan. LXXXV. Solutions of the boundary-layer equations. The London, Edinburgh, and Dublin Philosophical Magazine and Journal of Science, 12(80):865-896, 1931.

U. Fey and Y. Egami. Transition detection by temperature-sensitive paint. Springer Handbook of Experimental Fluid Mechanics, pages 537-552, 2007.

U. Fey, R. H. Engler, Y. Egami, Y. Iijima, K. Asai, U. Jansen, and J. Quest. Transition detection by temperature sensitive paint at cryogenic temperatures in the European Transonic Wind tunnel (ETW). In 20th International Congress on Instrumentation in Aerospace Simulation Facilities, 2003. ICIASF '03, pages 77-88. IEEE, 2003. doi:10.1109/ICIASF.2003.1274855.

U. Fey, Y. Egami, and C. Klein. Temperature-sensitive paint application in cryogenic wind tunnels: Transition detection at high reynolds numbers and influence of the technique on measured aerodynamic coefficients. In 22nd International Congress on Instrumentation in Aerospace Simulation Facilities (ICIASF), pages 1-17, 2007.

U. Fey, Y. Egami, R. Konrath, T. Kirmse, T. Ahlefeldt, and J. Kompenhans. Advanced measurement techniques for high Reynolds number testing in cryogenic wind tunnels. In 48th AIAA Aerospace Sciences Meeting Including the New Horizons Forum and Aerospace Exposition, 2010. 
U. Fey, C. Klein, V. Ondrus, S. Loose, and C. Wagner. Investigation of Reynolds number effects in high-speed train wind tunnel testing using temperature-sensitive paint. volume 44/2013, pages 29-47, Berlin, Germany, 2013. IFV Bahntechnik e.V.

D. L. Fisher and N. S. Dougherty, Jr. Flight and wind-tunnel correlation of boundary-layer transition on the AEDC transition cone. NASA STI/Recon Technical Report N, 83, Nov. 1982.

D. Fuciarelli, H. Reed, and I. Lyttle. Direct numerical simulation of leading-edge receptivity to sound. AIAA Journal, 38(7):1159-1165, 2000. doi:10.2514/2.1109.

M. Fujino, Y. Yoshizaki, and Y. Kawamura. Natural-laminar-flow airfoil development for a lightweight business jet. Journal of Aircraft, 40(4):609-615, 2003. doi:10.2514/1.168.

A. D. Gardner, C. Klein, W. E. Sachs, U. Henne, H. Mai, and K. Richter. Investigation of threedimensional dynamic stall on an airfoil using fast-response pressure-sensitive paint. Experiments in Fluids, 55(9):1807, Sept. 2014. doi:10.1007/s00348-014-1807-4.

G. L. Gluyas. Final report on LFC aircraft design data laminar flow control demonstration program. Technical Report NOR 67-136, Northrop Aircraft Inc., 1967.

M. Goldstein and L. Hultgren. Boundary-layer receptivity to long-wave freestream disturbances. Annual Review of Fluid Mechanics, 21:137-166, 1989. doi:10.1146/annurev.fl.21.010189.001033.

J. Green and J. Quest. A short history of the European Transonic Wind Tunnel ETW. Progress in Aerospace Sciences, 47(5):319-368, 2011. doi:10.1016/j.paerosci.2011.06.002.

J. W. Gregory, H. Sakaue, T. Liu, and J. P. Sullivan. Fast pressure-sensitive paint for flow and acoustic diagnostics. Annual Review of Fluid Mechanics, 46(1):303-330, Jan. 2014. doi:10.1146/annurev-fluid-010313-141304.

O. M. Haddad and T. C. Corke. Boundary layer receptivity to free-stream sound on parabolic bodies. Journal of Fluid Mechanics, 368:1-26, Aug. 1998. doi:10.1017/S0022112098001311.

P. W. Hammerton and E. J. Kerschen. Boundary-layer receptivity for a parabolic leading edge. Journal of Fluid Mechanics, 310:243-267, Mar. 1996. doi:10.1017/S0022112096001796.

K. Hannemann and J. Martinez Schramm. High enthalpy, high pressure short duration testing of hypersonic flows. In C. Tropea, J. Foss, and A. Yarin, editors, Springer Handbook of Experimental Fluid Mechanics, pages 1081-1125. Springer-Verlag Berlin Heidelberg, 2007.

K. Hannemann, J. Martinez Schramm, and S. Karl. Recent extensions to the High Enthalpy Shock Tunnel Göttingen (HEG). In 2nd International ARA Days, Arcachon, Frankreich, 2008.

R. Heinrich, M. Choudhari, and E. Kerschen. A comparison of boundary layer receptivity mechanisms. In 1st National Fluid Dynamics Conference. American Institute of Aeronautics and Astronautics, July 1988. doi:10.2514/6.1988-3758. 
C. Heister. Methodik zur näherungsweisen Vorhersage des laminar-turbulenten Umschlags an Hubschrauberrotoren. PhD thesis, 2016. URL http://dx.doi.org/10.18419/opus-8893.

T. Herbert. Parabolized stability equations. Annual Review of Fluid Mechanics, 29(1):245-283, 1997. doi:10.1146/annurev.fluid.29.1.245.

M. Hilfer, S. Dufhaus, D. Yorita, C. Klein, and A. Petersen. Application of pressure and temperature sensitive paints on a highly loaded turbine guide vane in a transonic linear cascade. In Global Power and Propulsion Society Forum, Zürich, Schweiz, 2017.

B. Hof, J. Westerweel, T. M. Schneider, and B. Eckhardt. Finite lifetime of turbulence in shear flows. Nature, 443(7107):59, Sept. 2006. doi:10.1038/nature05089

D. Hue, O. Vermeersch, D. Bailly, V. Brunet, and M. Forte. Experimental and numerical methods for transition and drag predictions of laminar airfoils. AIAA Journal, 53(9):2694-2712, Sept. 2015. doi $10.2514 / 1 . J 053788$.

D. Hue, O. Vermeersch, J. Duchemin, O. Colin, and D. Tran. Wind-tunnel and CFD investigations focused on transition and performance predictions of laminar wings. AIAA Journal, 56(1): 132-145, 2018. doi:10.2514/1.J056088.

R. D. Joslin. Overview of laminar flow control. Technical Report TP-1998-208705, NASA, 1998. URL https://ntrs .nasa.gov/search.jsp?R=19980232017.

C. Klein. Einsatz einer druckempfindlichen Beschichtung (PSP) zur Bestimmung des momentanen Druckfeldes von Modellen im Windkanal. Berichtsreihe, Georg-August-University, Göttingen, Germany, 1997. URL http://elib.dlr.de/13573/.

C. Klein, R. H. Engler, U. Henne, and W. E. Sachs. Application of pressure-sensitive paint for determination of the pressure field and calculation of the forces and moments of models in a wind tunnel. Experiments in Fluids, 39(2):475-483, Aug. 2005. doi:10.1007/s00348-005-1010-8.

C. Klein, U. Henne, W. Sachs, S. Hock, N. Falk, U. Beifuss, V. Ondrus, and S. Schaber. Pressure Measurement on Rotating Propeller Blades by means of the Pressure-Sensitive Paint Lifetime Method. In 51st AIAA Aerospace Sciences Meeting Including the New Horizons Forum and Aerospace Exposition, volume 2013-0483, Grapevine, Texas, USA, 2013. AIAA. doi: $10.2514 / 6.2013-483$.

C. Klein, U. Henne, W. Sachs, U. Beifuss, V. Ondrus, M. Bruse, R. Lesjak, and M. Löhr. Application of Carbon Nanotubes (CNT) and Temperature-Sensitive Paint (TSP) for the Detection of Boundary Layer Transition. In 52nd AIAA Aerospace Sciences Meeting, 2014-1482, Gaylord National, Maryland, USA, 2014.

E. J. Klein. Liquid crystals in aerodynamic testing. Astronautics \& Aeronautics, (6):70-73, 1968. URL https : //ntrs .nasa.gov/search.j.sp?R=19680053722 
S. Koch. Zeitliche und räumliche Turbulenzentwicklung in einem Rohrwindkanal und deren Einfluss auf die Transition an Profilmodellen. PhD Thesis, Georg-August-University, Göttingen, 2004.

P. Kolodner and J. A. Tyson. Remote thermal imaging with $0.7-\mu \mathrm{m}$ spatial resolution using temperature-dependent fluorescent thin flims. Applied Physics Letters, 42(1):117-119, Jan. 1983. doi:10.1063/1.93766.

L. S. G. Kovasznay. Turbulence in supersonic flow. Journal of the Aeronautical Sciences, 20(10): 657-674, 1953. doi:10.2514/8.2793.

S. Laurence, H. Ozawa, D. Lieber, J. Martinez Schramm, and K. Hannemann. Investigation of Unsteady/Quasi-Steady Scramjet Behavior using High-Speed Visualization Techniques. In 18th AIAA/3AF International Space Planes and Hypersonic Systems and Technologies Conference, Tours, France, Sept. 2012. American Institute of Aeronautics and Astronautics. doi:10.2514/6.2012-5913.

S. J. Laurence, A. Wagner, and K. Hannemann. Schlieren-based techniques for investigating instability development and transition in a hypersonic boundary layer. Experiments in Fluids, 55 (8):1782, Aug. 2014. doi:10.1007/s00348-014-1782-9.

J. Lemarechal, C. Klein, U. Henne, D. Puckert, and U. Rist. Transition delay by oblique roughness elements in a Blasius boundary-layer flow. In 2018 AIAA Aerospace Sciences Meeting, number 2018-1057. American Institute of Aeronautics and Astronautics, 2018. doi:10.2514/6.2018-1057.

H. W. Liepmann and G. H. Fila. Investigations of effects of surface temperature and single roughness elements on boundary-layer transition. NACA Technical Report 890, Jan. 1947.

N. Lin, H. L. Reed, and W. S. Saric. Effect of leading-edge geometry on boundary-layer receptivity to freestream sound. In Instability, Transition, and Turbulence, ICASE NASA LaRC Series, pages 421-440. Springer, New York, NY, 1992. doi:10.1007/978-1-4612-2956-8_42

T. Liu and J. P. Sullivan. Pressure and Temperature Sensitive Paints. Experimental Fluid Mechanics. Springer-Verlag Berlin Heidelberg, 2005.

L. M. Mack. Transition prediction and linear stability theory. In AGARD Conference Proceedings No 224 Laminar-Turbulent Transition, pages 1-2-1-22, Oct. 1977. URL http://adsabs harvard.edu/abs/1977ltt. .agarQ....M.

L. M. Mack. Boundary-layer linear stability theory. In AGARD Spec. Course on Stability and Transition of Laminar Flow, AGARD-R-709, pages 3-1-3-81, Rhode-Saint-Genèse, Belgium, June 1984.

J. Martinez Schramm, K. Hannemann, H. Ozawa, W. Beck, and C. Klein. Development of temperature sensitive paints in the High Enthalpy Shock Tunnel Göttingen, HEG. Lisbon, Portugal, Mar. 2015. 
J. A. Masad and Y. H. Zurigat. Effect of pressure gradient on first mode of instability in compressible boundary layers. Physics of Fluids, 6(12):3945-3953, Dec. 1994. doi:10.1063/1.868384.

W. D. McCauley, A. Saydah, and J. Bueche. The effect of controlled three dimensional roughness on hypersonic laminar boundary layer transition. AIAA Paper, 66:26, 1966.

H. U. Meier, U. Michel, and H.-P. Kreplin. The influence of wind tunnel turbulence on the boundary layer transition. In Perspectives in Turbulence Studies, pages 26-46. Springer, Berlin, Heidelberg, 1987. doi:10.1007/978-3-642-82994-9_2.

S. Melber-Wilkending, A. Heidebrecht, and G. Wichmann. CFD validation by wind tunnel measurements: Uncertainty assessment by numerical simulation of complete wind tunnel flows. In RTO-MP-AVT-147, Greece, 2007. doi:10.14339/RTO-MP-AVT-147. URL http://elib dlr.de/52152/.

F. Meyer and L. Kleiser. Numerical investigation of transition in 3D boundary layers. In AGARD, Fluid Dynamics of Three-Dimensional Turbulent Shear Flows and Transition (See N90-10356 01-34), Apr. 1989.

U. Michel and E. Froebel. Lower limit for the velocity fluctuation level in wind tunnels. Experiments in Fluids, 6(1):49-54, Jan. 1988. doi:10.1007/BF00226134

T. Nagayama, H. Nagai, H. Tanno, and T. Komuro. Global heat flux measurement using temperaturesensitive paint in High-Enthalpy Shock Tunnel HIEST. In 55th AIAA Aerospace Sciences Meeting, AIAA SciTech Forum. American Institute of Aeronautics and Astronautics, Jan. 2017. doi $10.2514 / 6.2017-1682$.

K. Nakakita, T. Osafune, and K. Asai. Global heat transfer measurement in a hypersonic shock tunnel using temperature-sensitive paint. In 41st Aerospace Sciences Meeting and Exhibit, Aerospace Sciences Meetings. American Institute of Aeronautics and Astronautics, Jan. 2003. doi: $10.2514 / 6.2003-743$.

S. Ohmi, H. Nagai, K. Asai, and K. Nakakita. Effect of TSP layer thickness on global heat transfer measurement in hypersonic flow. In 44th AIAA Aerospace Sciences Meeting and Exhibit, Aerospace Sciences Meetings. American Institute of Aeronautics and Astronautics, Jan. 2006. doi: $10.2514 / 6.2006-1048$.

V. Ondrus, R. J. Meier, C. Klein, U. Henne, M. Schäferling, and U. Beifuss. Europium 1,3di(thienyl)propane-1,3-diones with outstanding properties for temperature sensing. Sensors and Actuators A: Physical, 233:434-441, Sept. 2015. doi:10.1016/j.sna.2015.07.023.

W. M. Orr. The stability or instability of the steady motions of a perfect liquid and of a viscous liquid. Part II: A viscous liquid. In Proceedings of the Royal Irish Academy. Section A: Mathematical and Physical Sciences, volume 27, pages 69-138. JSTOR, 1907. 
H. Ozawa, S. J. Laurence, J. M. Schramm, A. Wagner, and K. Hannemann. Fast-response temperature-sensitive-paint measurements on a hypersonic transition cone. Experiments in Fluids, 56(1):1853, Jan. 2015. doi:10.1007/s00348-014-1853-y.

S. Özgen. Effect of heat transfer on stability and transition characteristics of boundarylayers. International Journal of Heat and Mass Transfer, 47(22):4697-4712, Oct. 2004. doi:10.1016/j.ijheatmasstransfer.2004.05.026.

R. K. Pachauri and L. Mayer, editors. Climate Change 2014: Synthesis Report. Contribution of Working Groups I, II and III to the Fifth Assessment Report of the Intergovernmental Panel on Climate Change. Intergovernmental Panel on Climate Change, Geneva, Switzerland, 2015.

D. Peng, L. Jiao, Z. Sun, Y. Gu, and Y. Liu. Simultaneous PSP and TSP measurements of transient flow in a long-duration hypersonic tunnel. Experiments in Fluids, 57(12):188, Dec. 2016. doi:10.1007/s00348-016-2280-z.

L. Prandtl. Über Flüssigkeitsbewegung bei sehr kleiner Reibung, Verhandlungen des dritten internationalen Mathematiker-Kongresses (Heidelberg 1904). pages 484-491, 1905. URL http://digital.library.unt.edu/ark:/67531/metadc65275.

H. L. Reed, W. S. Saric, and D. Arnal. Linear stability theory applied to boundary layers. Annual Review of Fluid Mechanics, 28:389-428, 1996. doi:10.1146/annurev.fl.28.010196.002133.

O. Reynolds. XXIX. An experimental investigation of the circumstances which determine whether the motion of water shall he direct or sinuous, and of the law of resistance in parallel channels. Philosophical Transactions of the Royal Society of London, 174:935-982, Jan. 1884. doi:10.1098/rstl.1883.0029.

S. Risius. Entwurf eines Messsystems zur instationären quantitativen TSP Messung am kryogenen Rohrwindkanal Göttingen (KRG). Berichtsreihe DLR-IB 224-2014 A 103, 2014. URL http: //elib.dlr.de/90744/

S. Risius. Application of TSP (Temperature Sensitive Paint) HiLamBiz (High Speed Wind Tunnel Test of a Laminar Configuration Bizjet) No. 338517, June 2016. URL https://www . etw. de/ publications/learn-more-movie/laminar-testing.

S. Risius, W. Sachs, and U. Henne. Know-how transfer towards the application of TemperatureSensitive Paint at the German-Dutch Wind Tunnels and the European Transonic Windtunnel. Berichtsreihe DLR-IB 224-2013 A 137, 2013. URL http://elib.dlr.de/83281/.

S. Risius, C. Fuchs, U. Henne, and C. Klein. Temperature-sensitive paint (TSP) results of the HINVA measurement campaign in the European Transonic Windtunnel (ETW). Berichtsreihe DLR-IB 224-2014 C 168, 2014a. URL http://elib.dlr.de/90743/.

S. Risius, U. Henne, and W. Sachs. Anleitungen für das TSP-Messsystem am European Transonic Windtunnel (ETW). Berichtsreihe DLR-IB 224-2014 A 105, 2014b. URL http://elib.dlr de/90739/. 
S. Risius, M. Costantini, and T. Kleindienst. Untersuchung optischer Komponenten (LEDs und Filter) für den Einsatz bei kryoTSP und warmTSP. Berichtsreihe 224-2015 A 113, 2015a. URL http://elib.dlr.de/98739/

S. Risius, H. Xu, F. Di Lorenzo, H. Xi, H. Siebert, R. A. Shaw, and E. Bodenschatz. Schneefernerhaus as a mountain research station for clouds and turbulence; Part 1: Flow conditions and large-scale turbulence. Atmos. Meas. Tech., 8(8):3209-3218, Aug. 2015b. doi:10.5194/amt-83209-2015.

S. Risius, W. H. Beck, C. Klein, U. Henne, and A. Wagner. Determination of heat transfer into a wedge model in a hypersonic flow using temperature-sensitive paint. Experiments in Fluids, 58 (9):117, Sept. 2017. doi:10.1007/s00348-017-2393-z.

S. Risius, M. Costantini, S. Hein, S. Koch, and C. Klein. Experimental investigation of Mach number and pressure gradient effects on boundary layer transition in two-dimensional flow. In New Results in Numerical and Experimental Fluid Mechanics XI, Notes on Numerical Fluid Mechanics and Multidisciplinary Design, pages 305-314. Springer, Cham, 2018a. doi:10.1007/978-3-31964519-3_28.

S. Risius, M. Costantini, S. Koch, S. Hein, and C. Klein. Unit Reynolds number, Mach number and pressure gradient effects on laminar-turbulent transition in two-dimensional boundary layers. Experiments in Fluids, 59(86), May 2018b. doi:10.1007/s00348-018-2538-8.

H. Rosemann. The Cryogenic Ludwieg-Tube Tunnel at Göttingen. In Special Course on Advances in Cryogenic Wind Tunnel Technology, number R-812 in AGARD, pages 8-1-8-13. Neuilly-surSeine, 1997.

R. Rudnik, N. F. Bier, J. Quest, and M. Schulz. Flight Test Tailored ETW Testing of a Commercial Transport High Lift Configuration. In 53rd AIAA Aerospace Sciences Meeting. American Institute of Aeronautics and Astronautics, Jan. 2015. doi 10.2514/6.2015-1559

W. Saric and E. White. Influence of high-amplitude noise on boundary-layer transition to turbulence. In 29th AIAA, Fluid Dynamics Conference, number 1998-2645 in Fluid Dynamics and co-located Conferences. American Institute of Aeronautics and Astronautics, June 1998. doi $10.2514 / 6.1998-2645$.

W. Saric, E. White, and H. Reed. Boundary-layer receptivity to freestream disturbances and its role in transition. In 30th Fluid Dynamics Conference, number 1999-3788. American Institute of Aeronautics and Astronautics, 1999. doi:10.2514/6.1999-3788

H. Schlichting. Zur Enstehung der Turbulenz bei der Plattenströmung. Nachrichten von der Gesellschaft der Wissenschaften zu Göttingen, Mathematisch-Physikalische Klasse, 1933:181208, 1933.

H. Schlichting and K. Gersten. Boundary-Layer Theory. Springer, Berlin, New York, 8th edition edition, Mar. 2000. 
G. Schrauf. Transition prediction using different linear stability analysis strategies. In 12th Applied Aerodynamics Conference, number 1994-1848 in Fluid Dynamics and co-located Conferences, pages 367-375. American Institute of Aeronautics and Astronautics, June 1994. doi:10.2514/6.1994-1848.

G. Schrauf. COCO-A program to compute velocity and temperature profiles for local and nonlocal stability analysis of compressible, conical boundary layers with suction. ZARM Technik report, 1998.

G. Schrauf. Evaluation of the A320 hybrid laminar fin experiment. In European Congress on Computational Methods in Applied Sciences and Engineering, ECCOMAS, Barcelona, Spain, Sept. 2000.

G. Schrauf. Status and perspectives of laminar flow. The Aeronautical Journal, 109(1102):639-644, Dec. 2005. doi:10.1017/S000192400000097X.

G. Schrauf. LILO 2.1 User's guide and tutorial. Bremen, Germany, GSSC Technical Report, 6, 2006.

G. Schrauf, T. Herbert, and G. Stuckert. Evaluation of transition in flight tests using nonlinear parabolized stability equation analysis. Journal of Aircraft, 33(3):554-560, May 1996. doi:10.2514/3.46980.

G. Schrauf, J. Perraud, D. Vitiello, and F. Lam. Comparison of boundary-layer transition predictions using flight test data. Journal of Aircraft, 35(6):891-897, Nov. 1998. doi:10.2514/2.2409.

G. B. Schubauer and H. K. Skramstad. Laminar-boundary-layer oscillations and transition on a flat plate. Technical Report NACA-TR-909, NASA, Jan. 1948. Also bound with NACA Annual Report 34, p. 327-357.

D. L. Schultz and T. V. Jones. Heat-transfer measurements in short-duration hypersonic facilities. Technical Report AGARD-AG-165, Advidsory Group for Aerospace Research and Development, Paris, France, Feb. 1973.

N. Shahriari, D. J. Bodony, A. Hanifi, and D. S. Henningson. Acoustic receptivity simulations of flow past a flat plate with elliptic leading edge. Journal of Fluid Mechanics, 800, Aug. 2016. doi:10.1017/jfm.2016.433.

H. Siebert, R. A. Shaw, J. Ditas, T. Schmeissner, S. P. Malinowski, E. Bodenschatz, and H. Xu. High-resolution measurement of cloud microphysics and turbulence at a mountaintop station. Atmos. Meas. Tech., 8(8):3219-3228, Aug. 2015. doi:10.5194/amt-8-3219-2015.

A. M. O. Smith and N. Gamberoni. Transition, pressure gradient, and stability theory. Technical Report ES. 26388, Douglas Aircraft Co Inc, El Segundo, CA, 1956.

E. Softley, B. C. Graber, and R. E. Zempel. Experimental observation of transition of the hypersonic boundary layer. AIAA Journal, 7(2):257-263, 1969. doi:10.2514/3.5083 
A. Sommerfeld. Ein Beitrag zur hydrodynamischen Erklärung der turbulenten Flüssigkeitsbewegung. In G. Castelnuovo, editor, Atti Del IV Congresso Internazionale Dei Matematici, volume 3, pages 116-124, Rome, 1908.

H. B. Squire. On the stability for three-dimensional disturbances of viscous fluid flow between parallel walls. Proc. R. Soc. Lond. A, 142(847):621-628, 1933. doi 10.1098/rspa.1933.0193.

P. Stainback. Some effects of roughness and variable entropy on transition at a Mach number of 8. In 5th Aerospace Sciences Meeting. American Institute of Aeronautics and Astronautics, Jan. 1967. doi:10.2514/6.1967-132.

P. C. Stainback, R. D. Wagner, F. K. Owen, and C. C. Horstmann. Experimental studies of hypersonic boundary-layer transition and effects of wind-tunnel disturbances. Technical Report NASA TN D-7453, National Aeronautics and Space Administration, 1974.

K. Stetson, E. Thompson, J. Donaldson, and L. Siler. Laminar boundary layer stability experiments on a cone at Mach 8. IV- On unit Reynolds number and environmental effects. In 4th Joint Fluid Mechanics, Plasma Dynamics and Lasers Conference. American Institute of Aeronautics and Astronautics, May 1986. doi:10.2514/6.1986-1087.

T. Streit, K. Horstmann, G. Schrauf, S. Hein, U. Fey, Y. Egami, J. Perraud, I. S. El Din, U. Cella, and J. Quest. Complementary numerical and experimental data analysis of the ETW Telfona pathfinder wing transition tests. In 49th AIAA Aerospace Sciences Meeting Including the New Horizons Forum and Aerospace Exposition. American Institute of Aeronautics and Astronautics, Jan. 2011. doi:10.2514/6.2011-881

A. Styles and S. Risius. Assessment and validation of aerodynamic performance results for a natural laminar flow transonic wing tested in cryogenic conditions via simulation of turbulent wedges in CFD. Bristol, England, 2016. URL http://elib.dlr.de/105719/

W. Tollmien. Über die Entstehung der Turbulenz. 1. Mitteilung. Nachrichten von der Gesellschaft der Wissenschaften zu Göttingen, Mathematisch-Physikalische Klasse, 1929:21-44, 1928.

C. Tropea, A. L. Yarin, and J. F. Foss, editors. Springer Handbook of Experimental Fluid Mechanics. Springer handbooks. Springer, Berlin, 2007.

J. van Ingen. A suggested semi-empirical method for the calculation of the boundary layer transition region. Technical Report Rapport VTH-74, Technische Hogeschool Delft, Vliegtuigbouwkunde, Delft, 1956.

J. van Ingen. The eN Method for transition prediction. Historical review of work at TU Delft. In 38th Fluid Dynamics Conference and Exhibit, number 2008-3830 in Fluid Dynamics and co-located Conferences. American Institute of Aeronautics and Astronautics, June 2008. doi: $10.2514 / 6.2008-3830$. 
A. Vollan and L. Alati. A new optical pressure measurement system (OPMS). In International Congress on Instrumentation in Aerospace Simulation Facilities, pages 10-16, 1991. doi:10.1109/ICIASF.1991.186219.

N. Voogt. Flight testing of a Fokker 100 test aircraft with laminar flow glove. In 2nd European Forum on Laminar Flow Technology, volume 2.1, pages 2-3-2-14, Paris, France, 1996.

A. Wagner, M. Kuhn, J. M. Schramm, and K. Hannemann. Experiments on passive hypersonic boundary layer control using ultrasonically absorptive carbon-carbon material with random microstructure. Experiments in Fluids, 54(10):1606, Oct. 2013. doi:10.1007/s00348-013-1606-3.

J. Wild. Simulation and flight Re-number testing of high-lift systems. In 6th European Conference on Computational Fluid Dynamics, Barcelona, Spanien, 2014.

W. Würz, D. Sartorius, M. Kloker, V. I. Borodulin, Y. S. Kachanov, and B. V. Smorodsky. Detuned resonances of Tollmien-Schlichting waves in an airfoil boundary layer: Experiment, theory, and direct numerical simulation. Physics of Fluids, 24(9):094103, Sept. 2012a. doi:10.1063/1.4751246.

W. Würz, D. Sartorius, M. Kloker, V. I. Borodulin, Y. S. Kachanov, and B. V. Smorodsky. Nonlinear instabilities of a non-self-similar boundary layer on an airfoil: Experiments, DNS, and theory. European Journal of Mechanics - B/Fluids, 31:102-128, Jan. 2012b. doi:10.1016/j.euromechflu.2011.07.006.

Y. H. Zurigat, A. H. Nayfeh, and J. A. Masad. Effect of pressure gradient on the stability of compressible boundary layers. AIAA Journal, 30(9):2204-2211, Sept. 1992. doi:10.2514/3.11206. 


\section{Part IV}

\section{Appendices}

A Further results

B Principles of temperature-sensitive paint and advances in the measurement system

?? Curriculum Vitae 



\section{Appendix A}

\section{Further results}

The first part of the Appendix shows the results of the transition Reynolds number analysis obtained for the Mach numbers $M=0.50$ and $M=0.65$ (Fig. A.1 - Fig. A.4). The second part shows incompressible and compressible critical $N$-factors of the first wind tunnel entry for each Mach number separately (Fig. A.5- Fig. A.10).
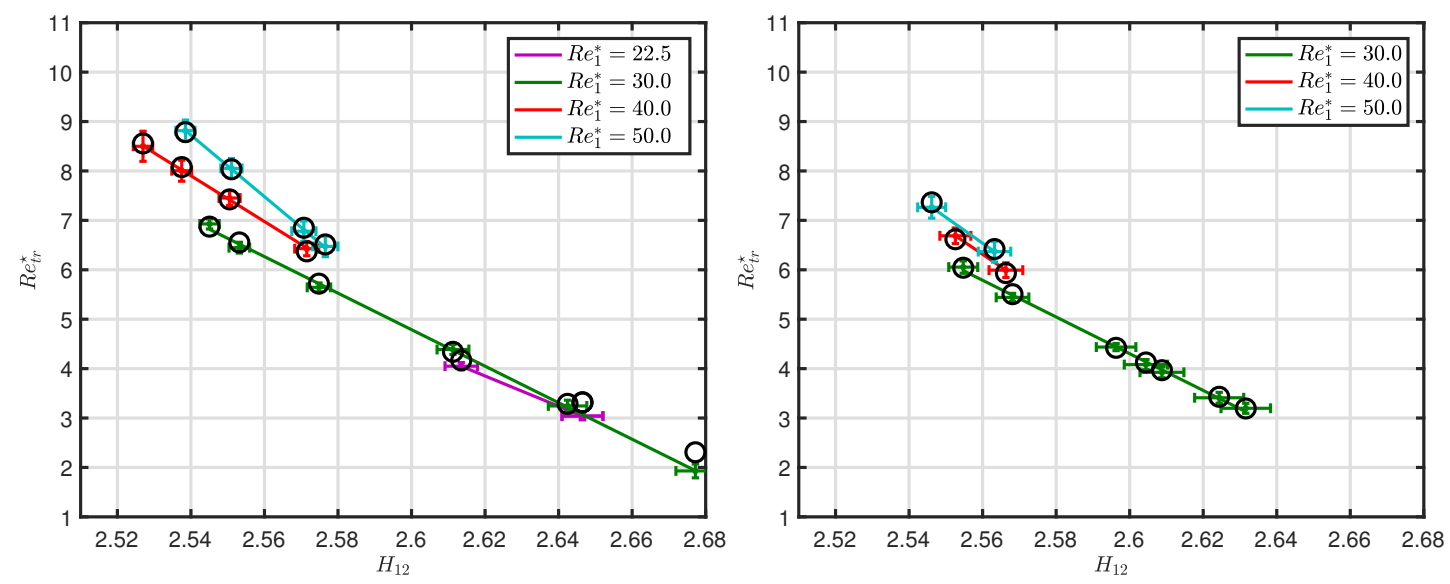

Figure A.1: Transition Reynolds number as a function of the incompressible shape factor, $H_{12}$, for different unit Reynolds numbers at $M=0.50$ (left) and $M=0.65$ (right). The vertical and horizontal error bars are RMS values of the transition location variation along the span (Fig. 5.3) and the chordwise shape factor approximation (Fig. 5.5), respectively. Black circles mark the calculated transition Reynolds numbers with the help of Eq. [III 

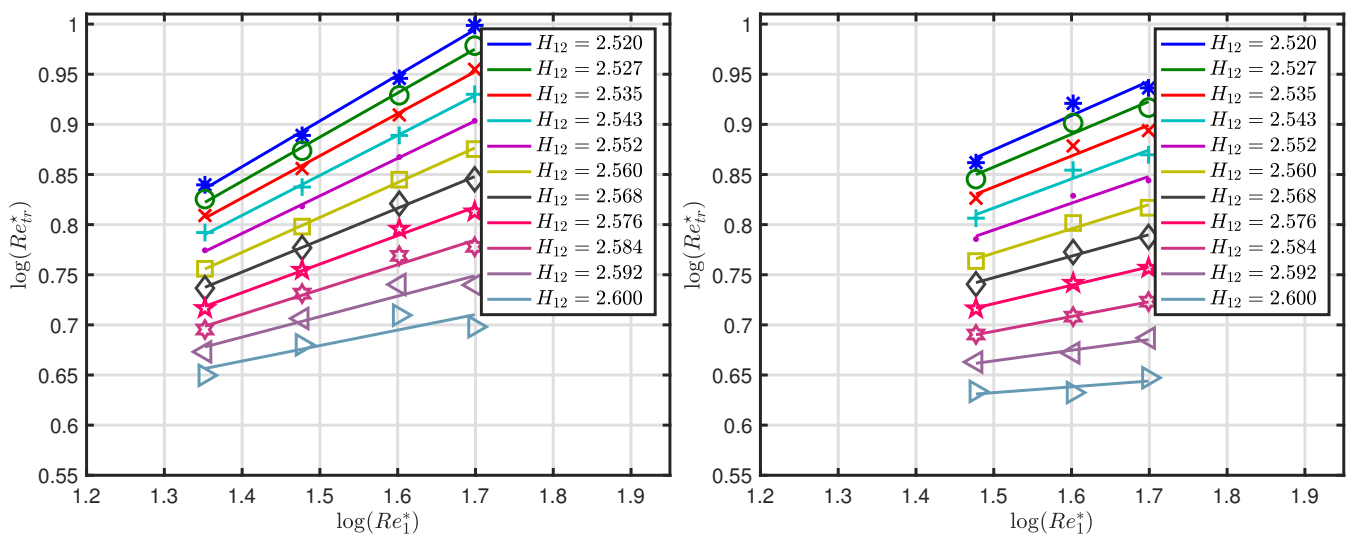

Figure A.2: Transition Reynolds number as a function of unit Reynolds number in a double logarithmic plot for different values of $H_{12}$ at $M=0.50$ (left) and $M=0.65$ (right). The symbols correspond to transition Reynolds numbers computed with Eq. II for different values of $R e_{1}^{\star}$
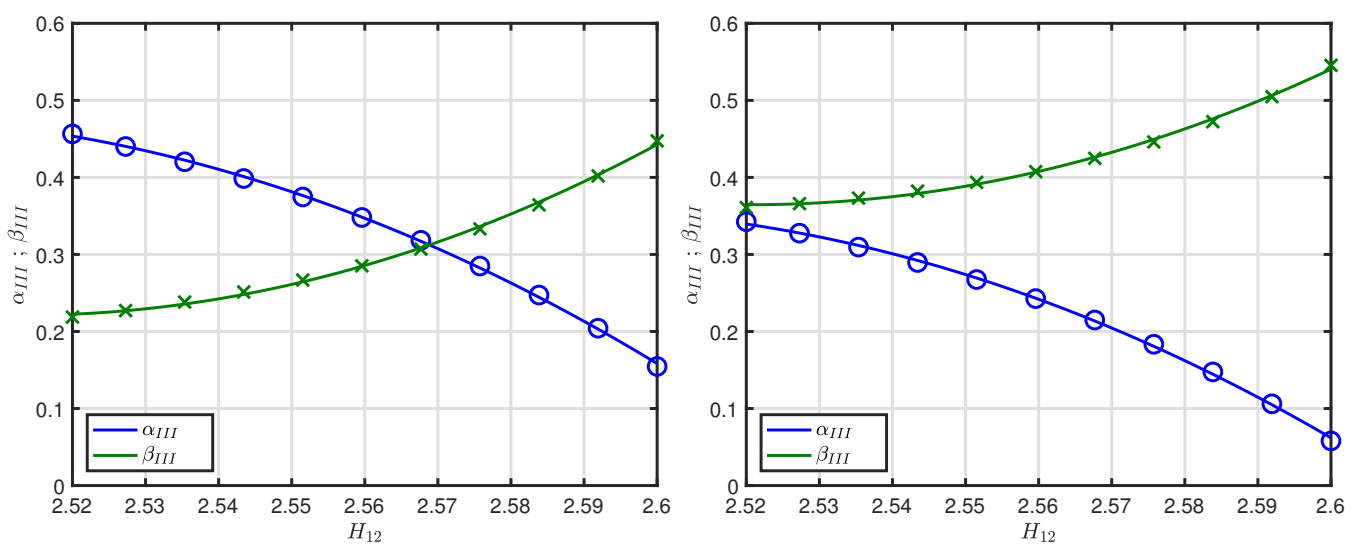

Figure A.3: Coefficients $\alpha_{\text {III }}$ and $\beta_{\text {III }}$ as a function of $H_{12}$ at $M=0.50$ (left) and $M=0.65$ (right) with approximations based on quadratic functions with $\alpha_{\mathrm{III}}=h_{\mathrm{III}, \alpha, 2} \cdot H_{12}^{2}+h_{\mathrm{III}, \alpha, 1}$. $H_{12}+h_{\mathrm{III}, \alpha, 0}$ and $\beta_{\mathrm{III}}=h_{\mathrm{III}, \beta, 2} \cdot H_{12}^{2}+h_{\mathrm{III}, \beta, 1} \cdot H_{12}+h_{\mathrm{III}, \beta, 0}$ 

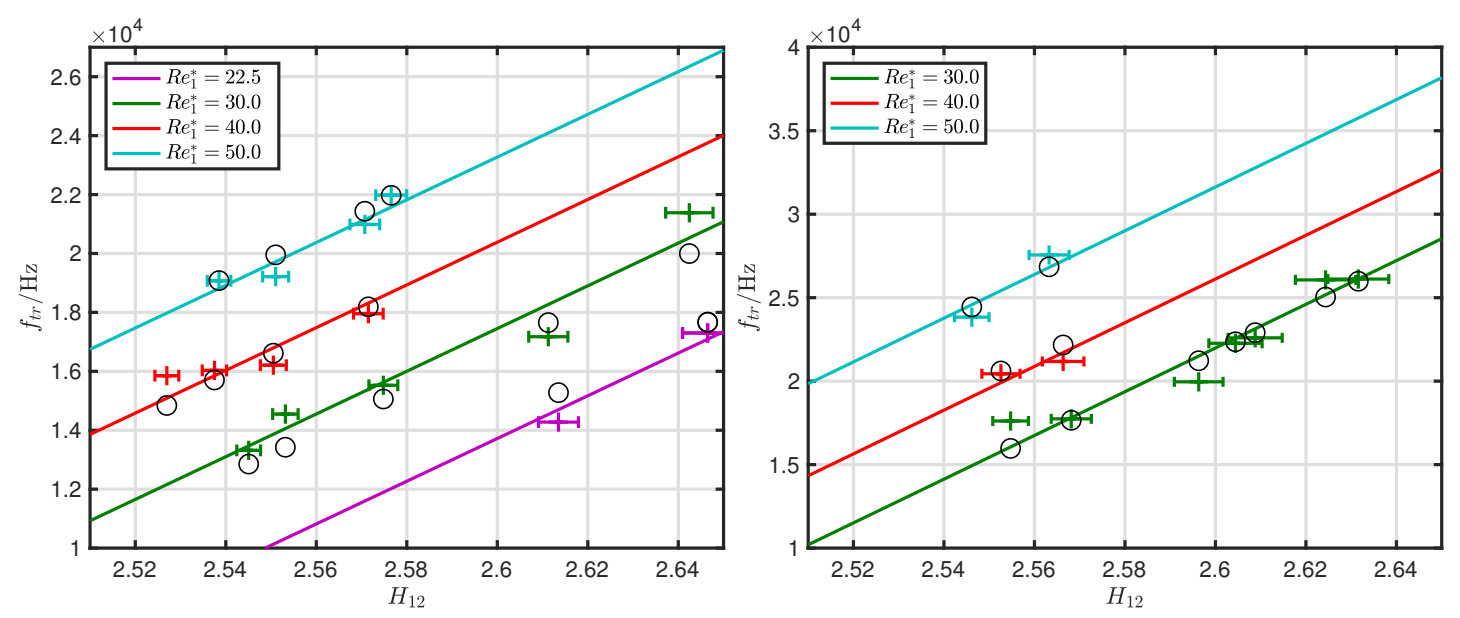

Figure A.4: Frequency of the most amplified T-S wave at the transition location as a function of $H_{12}$ for different values of unit Reynolds numbers at $M=0.50$ (left) and $M=0.65$ (right). Error bars are RMS values of the $H_{12}$ approximation as shown in Fig. 5.5. For comparison the black circles visualize the approximated value of $f_{t r}$ with the help of Eq. IV 

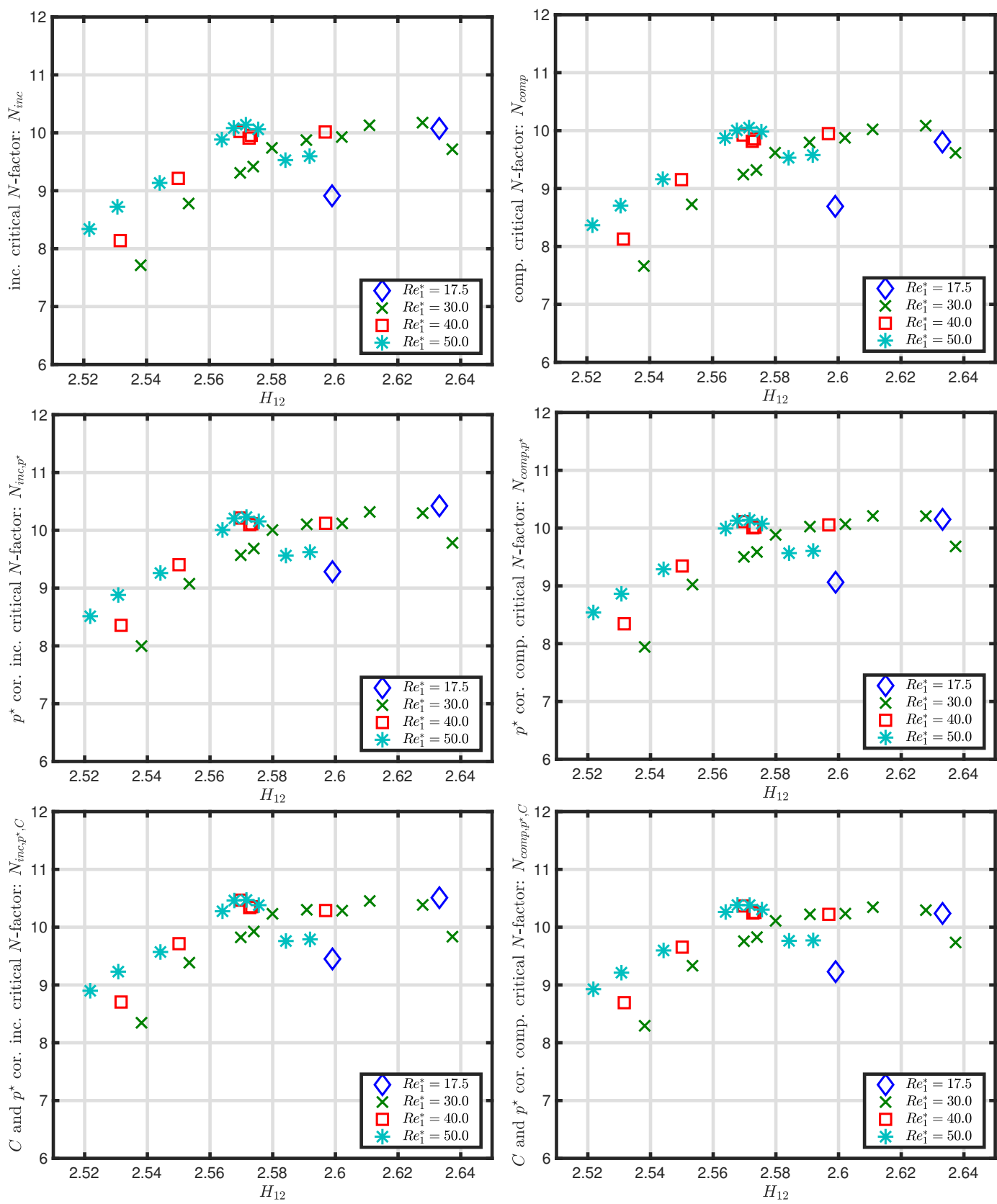

Figure A.5: Incompressible critical $N$-factors, Figure A.6: Compressible critical $N$-factors,

$N_{i n c}, N_{i n c, p^{\star}}$ and $N_{i n c, p^{\star}, C}$ (from top to bottom) as a function of $H_{12}$ at $M=0.35$
$N_{\text {comp }}, N_{\text {comp }, p^{\star}}$ and $N_{\text {comp }, p^{\star}, C}$ (from top to bottom) as a function of $H_{12}$ at $M=0.35$ 

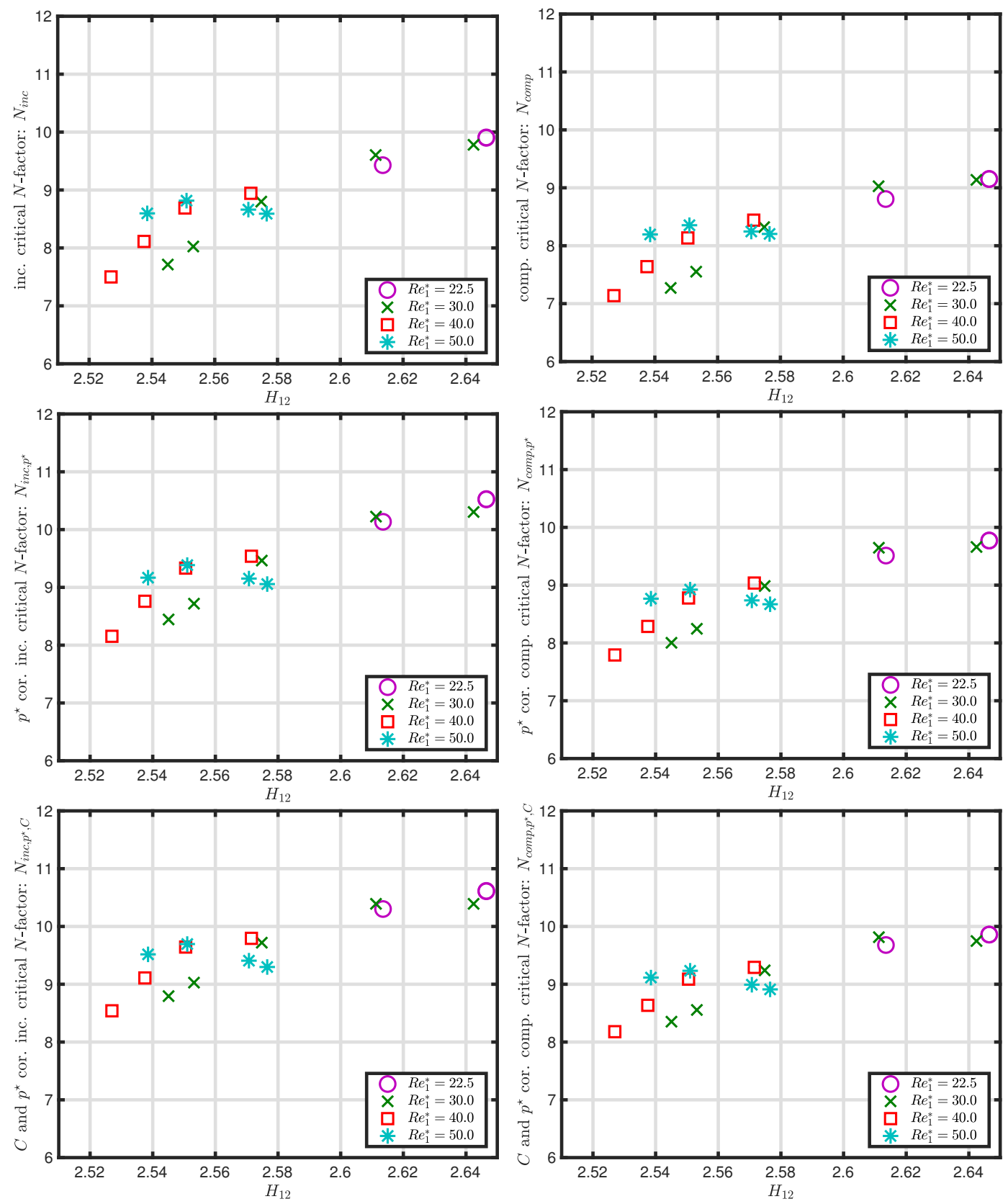

Figure A.7: Incompressible critical $N$-factors, Figure A.8: Compressible critical $N$-factors, $N_{i n c}, N_{i n c, p^{\star}}$ and $N_{i n c, p^{\star}, C}$ (from $N_{\text {comp }}, N_{\text {comp }, p^{\star}}$ and $N_{\text {comp }, p^{\star}, C}$ top to bottom) as a function of $H_{12}$ at $M=0.50$ (from top to bottom) as a function of $H_{12}$ at $M=0.50$ 

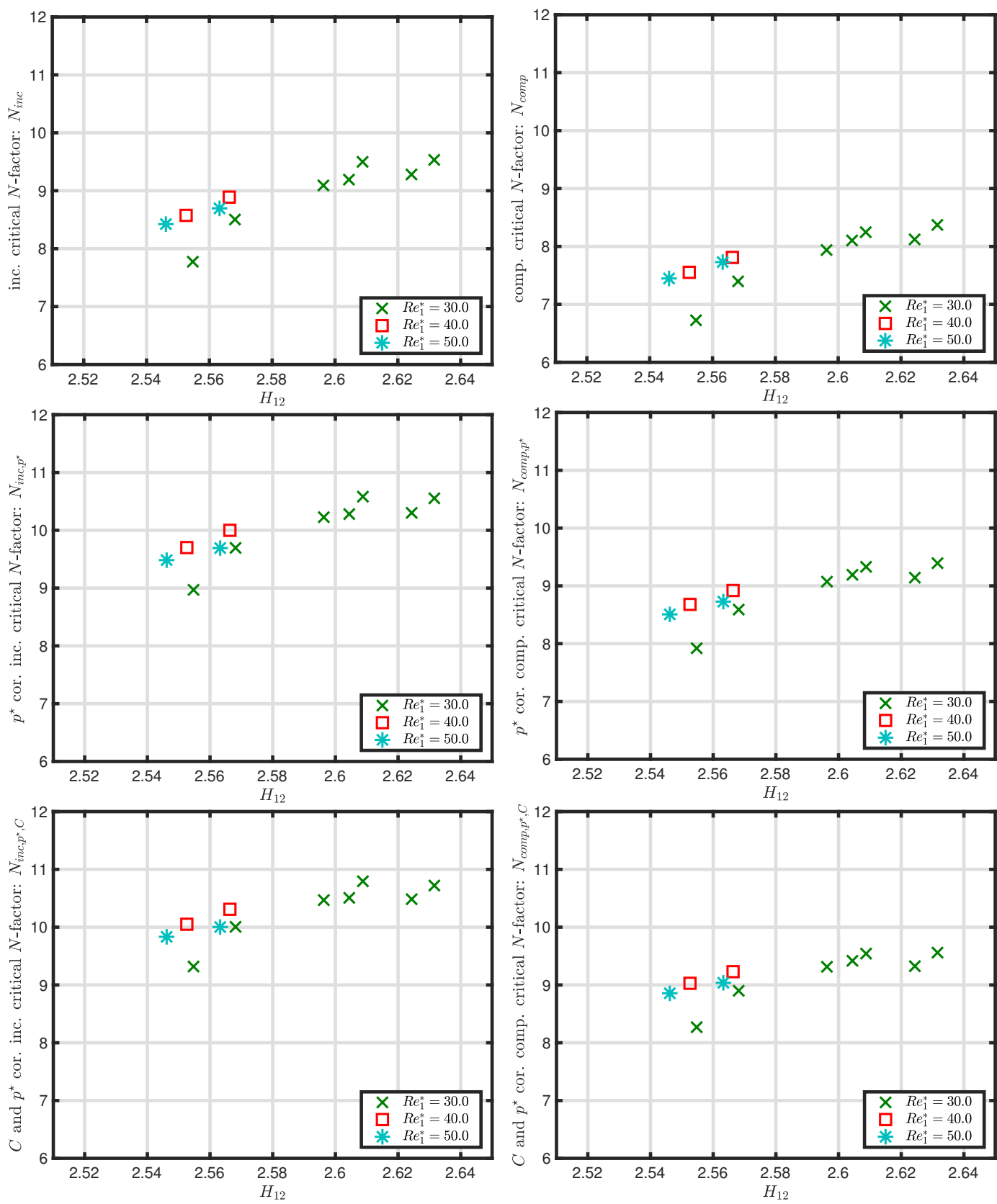

Figure A.9: Incompressible critical $N$-factors, Figure A.10: Compressible critical $N$-factors,

$N_{i n c}, N_{i n c, p^{\star}}$ and $N_{i n c, p^{\star}, C}$ (from top to bottom) as a function of $H_{12}$ at $M=0.65$
$N_{\text {comp }}, N_{\text {comp }, p^{\star}}$ and $N_{\text {comp }, p^{\star}, C}$ (from top to bottom) as a function of $H_{12}$ at $M=0.65$ 


\title{
Appendix B
}

\section{Principles of temperature-sensitive paint and advances in the measurement system}

\author{
B.1 Thermographic measurements and the \\ temperature-sensitive paint (TSP) technique ${ }^{1}$
}

The temperature distribution on a surface can be measured by various methods. Thermographic phosphors and thermochromic liquid crystals have been applied already in the 1960s for aerodynamic testing (Czysz and Dixon, 1969; Klein, 1968). The infrared thermography technique has been used since the 1980s for flow visualization and non-intrusive temperature and heat flux measurements (eg. de Luca et al. (1990)). In the 1980s also the polymer-based TSPs were developed by Kolodner and Tyson (1983) who used a Europium-based TSP to measure the surface temperature distribution of an integrated circuit.

For TSP applications, temperature-sensitive molecules are embedded in a polymer-based paint which is coated on the investigated surface. The molecules are excited with light of a proper wavelength causing the molecules to go into an excited electronic state. The molecules go from the excited state back into the ground state through radiation of light (luminescence) or a radiationless process. The basic principle of TSP is the process of thermal quenching, which refers to a decreasing quantum efficiency of luminescence with increasing temperature, where the relation between the luminscent intensity $I$ and absolute temperature $T$ can be written in an Arrhenius form of Eq. B.4.

\section{B.1.1 Basics of photophysical processes}

The electronic states of the temperature sensitive molecule can be symbolized by a Jablonski diagram, which is used to visualize the transition of valence electrons between different quantum states. Valence electrons are elevated to a higher energy state by a photon of the energy $E=h \cdot f$, with the Planck constant $h$ and the frequency $f$. Afterwards the valence electron can fall back into the ground state via several different processes:

- vibrational relaxation or external conversion without emission of light

- internal conversion to a lower electronic state (of same multiplicity) without emission of light

\footnotetext{
${ }^{1}$ This description of the general TSP technique follows in many parts the line of thought of Liu and Sullivan (2005)
} 
- intersystem crossing to a lower electronic state (of different multiplicity) without emission of light

- radiative transition with the emission of light (referred to as luminescence):

- spin-allowed radiative transition between two states of same multiplicity (referred to as fluorescence)

- spin-forbidden radiative transition from a triplet state to a singlet state (referred to as phosphorescence)

Since phosphorescence is a forbidden transition, the lifetime of a phosphorescent process is usually longer than the lifetime of a fluorescent process. The general term for both processes is luminescence.

\section{B.1.2 Quenching}

The luminescence intensity of the molecules can be decreased by a variety of processes, referred to as quenching, which causes a higher amount of molecules to go back into the ground state without the emission of light. Two main types of quenching can be distinguished:

- dynamic quenching: the energy of the excited molecule is transmitted to the quencher molecule by collision.

- static quenching: the excited molecule and the quencher molecule form a chemical complex which has a lower luminescence.

Two special cases of dynamic quenching are most relevant for aerodynamic testing: oxygen quenching and thermal quenching. Due to oxygen quenching the air pressure on an aerodynamic model surface can be related to the luminescent intensity via the Stern-Volmer equation, which is the major photophysical mechanism for pressure-sensitive paint (Liu and Sullivan, 2005). Thermal quenching is caused by an increased frequency of molecule collisions at higher temperatures leading to an increased rate of external conversion, which is the major photophysical mechanism for TSP described by the Arrhenius equation (Liu and Sullivan, 2005).

\section{B.1.3 The Arrhenius equation}

According to Liu and Sullivan (2005) the quantum yield of luminescence is given by

$$
\Phi=\frac{I}{I_{a}} \frac{k_{r}}{k_{r}+k_{n r}}
$$

$I$ and $I_{a}$ refer to the luminescent intensity and the rate of excitation, respectively, while the coefficients $k_{r}$ and $k_{n r}$ correspond to the rate constant for radiation process and the combined effect of all non-radiative processes, respectively. The coefficient $k_{n r}$ can be written as a combination of a temperature-independent rate constant, $k_{n r 1}$, and a temperature-dependent rate constant, $k_{n r 0}$ :

$$
k_{n r}=k_{n r 0}+k_{n r 1} \exp \left(-\frac{E_{n r}}{R T}\right)
$$


In Eq. B.2 the factor $R$ is the universal gas constant, $T$ is the absolute temperature in Kelvin and $E_{n r}$ is the activation energy for the non-radiative process. Eq. B.1 and Eq. B.2 can be combined to give

$$
\ln \left(\frac{I(T)\left[I_{0}-I\left(T_{r e f}\right)\right]}{I\left(T_{r e f}\right)\left[I_{0}-I(T)\right]}\right)=\frac{E_{n r}}{R}\left(\frac{1}{T}-\frac{1}{T_{r e f}}\right)
$$

where $I_{0}=I(T=0)$ is the luminescence intensity at $T=0$ (Liu and Sullivan, 2005). For $\frac{\left\|I(T)-I\left(T_{\text {ref }}\right)\right\|}{I_{0}} \ll 1$ and $\frac{I(T) I\left(T_{\text {ref }}\right)}{I_{0}^{2}} \ll 1$ a relation between the luminescence intensity and temperature can be written in an Arrhenius form (Liu and Sullivan, 2005):

$$
\ln \left(\frac{I(T)}{I\left(T_{r e f}\right)}\right)=\frac{E_{n r}}{R}\left(\frac{1}{T}-\frac{1}{T_{r e f}}\right)
$$

\section{B.1.4 Temperature sensitive molecules}

In this study a Europium (Eu) complex (Fig. B.1 left) is used as luminophore in TSP which is described in detail by Ondrus et al. (2015). The molecules are characterized by an excitation peak at $\lambda_{\text {ex }} \approx 410 \mathrm{~nm}$ and a peak of emission at $\lambda_{\text {ex }} \approx 614 \mathrm{~nm}$ (Fig. B.1 right). In order to determine the temperature and pressure sensitivity of the TSP, small test samples are coated with TSP and calibrated in a calibration chamber (see Sect. B.2.1). The normalized intensity and the negative relative temperature sensitivity are shown in Fig. B.2. It can be seen that the intensity of the TSP decreases with increasing temperature; this is caused by the increasing rate of thermal quenching with increasing temperatures (see Sects. B.1.1 and B.1.3.
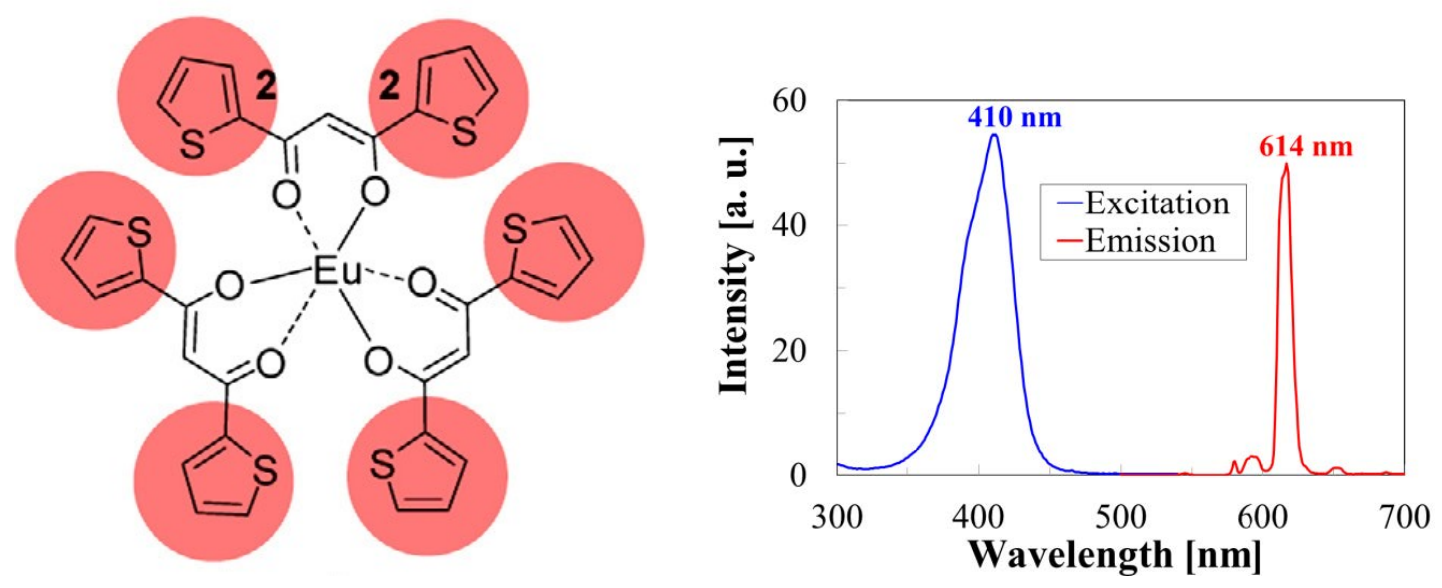

Figure B.1: Left: Example structures of the Europium complex used as luminophore in this study. (By courtesy of Elsevier from Ondrus et al. (2015)). Right: Excitation and emission spectra $\left(\lambda_{e x}=405 \mathrm{~nm}\right)$ of Ruthenium complex in a three-component polyurethane (3C-PU) with a PU-based screen layer at $30^{\circ} \mathrm{C}$ and $100 \mathrm{kPa}$ (By courtesy of Costantini (2016); Ondrus et al. (2015)) 

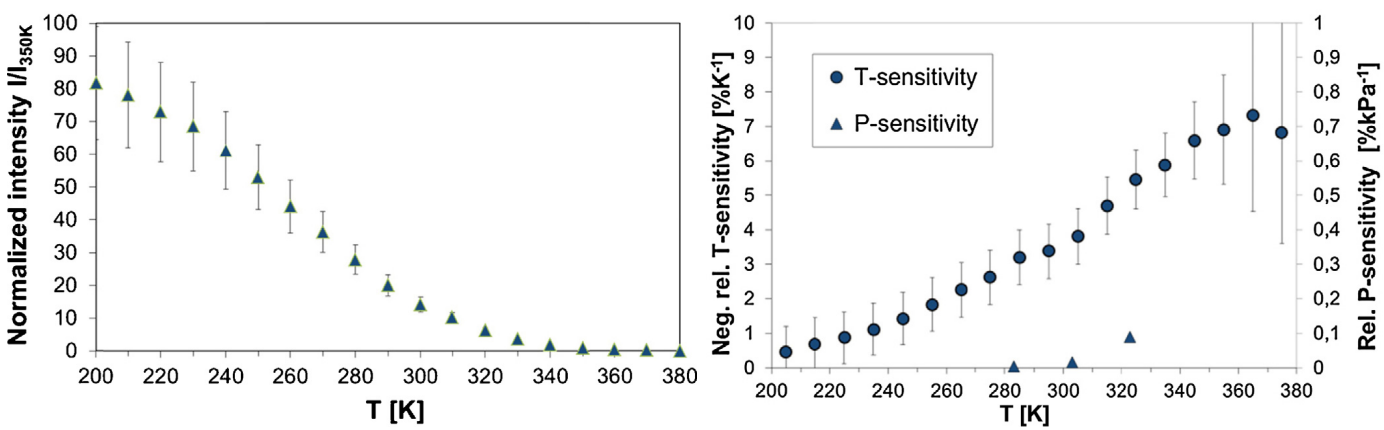

Figure B.2: Normalized luminescence intensity with respect to its value at $350 \mathrm{~K}$ (left) and relative temperature and pressure sensitivity (right) as a function of temperature in 3C-PU. Standard deviations are shown as error bars. (By courtesy of Elsevier from Ondrus et al. (2015))

\section{B.2 Setup for TSP data acquisition}

The general setup which is required for TSP measurements consists of the following devices:

- light source

- scientific camera (CCD or CMOS)

- optical filters for cameras and LEDs

- trigger device for synchronization of data acquisition

- PC for data processing and storage

- model coated with TSP

An overview over an exemplary setup is shown in Fig. B.3. More detailed descriptions of the TSP measurement technique are given in Tropea et al. (2007) and Liu and Sullivan (2005). Important improvements made for TSP measurements are described in the following sections.

\section{B.2.1 Calibration chamber for TSP}

In order to conduct a calibration of the TSP a calibration chamber was used which allows the controlled adjustment of temperature and pressure for a small TSP test sample (Egami et al., 2012). A schematic setup for the calibration of TSP is shown in Fig. B.4. The optical components (LEDs and filters) used for TSP calibration are the same as the components used in the wind tunnel measurements.

\section{B.2.2 LEDs}

One possible way to improve the TSP luminescence intensity (and along the signal-to-noise ratio of the result images) is to increase the intensity of the excitation light. Over the past years LED light sources have advanced quickly, leading to LEDs with a significantly larger intensity. In order to improve the signal-to-noise ratio of TSP result images, a variety of LEDs were tested and compared (Risius et al. 2015a). Before the improvements LEDs denoted as ACULED-VHL were used for TSP 


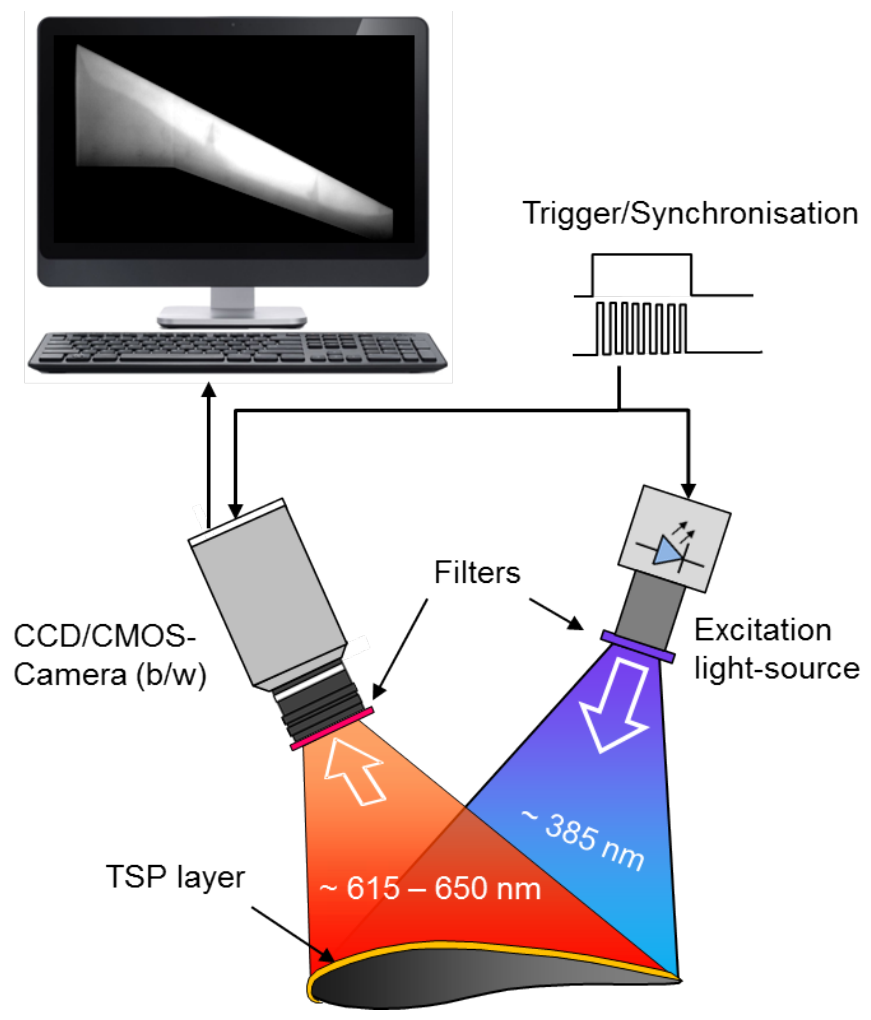

Figure B.3: Schematic setup of the TSP measurement system. Image by courtesy of Hilfer et al. (2017)

measurements in DNW-KRG (e.g. Costantini et al. (2012); Fey et al. (2010)). It was found that the best intensity gain was achieved with LEDs denoted as LED ENGIN LZ4-40UA00-U8. Therefore, these new LEDs were installed in DNW-KRG. The normalized emission spectrum of both LEDs is comparable, as shown in Fig. B.5 (Risius et al., 2015a). However, the absolute intensity of the LEDs LED ENGIN LZ4-40UAOO-U8 is significantly larger, leading also to a higher luminescence intensity of TSP (not shown here).

\section{B.2.3 Optical filters}

In order to reduce the transmitted wavelengths, optical filters have to be installed in front of the light sources and cameras for TSP measurements. Due to new manufacturing techniques, improved optical filters have become available over the last years. Therefore, optical filters suitable for TSP measurement were also tested and improved by Risius et al. (2015a). The old filters in front of the cameras, denoted as 590FG05, were replaced by the new filters ET590LP. Also, the old filters in front of the LEDs D415/60M were replaced by filters, denoted as ET410/40X. The spectra of the camera filters and LED filters are compared in Figs. B.6 and B.7, respectively. It can be seen that the wavelengths of the transmission spectra is comparable, but the transmission of the new filters is significantly larger, leading to an increased intensity of TSP results. Also the new filters have sharper cut-off frequency which avoids transmission of unwanted wavelengths more effectively. The absolute intensity of LEDs and filters are compared in Figs. B.8 and B.9. It can be seen that 

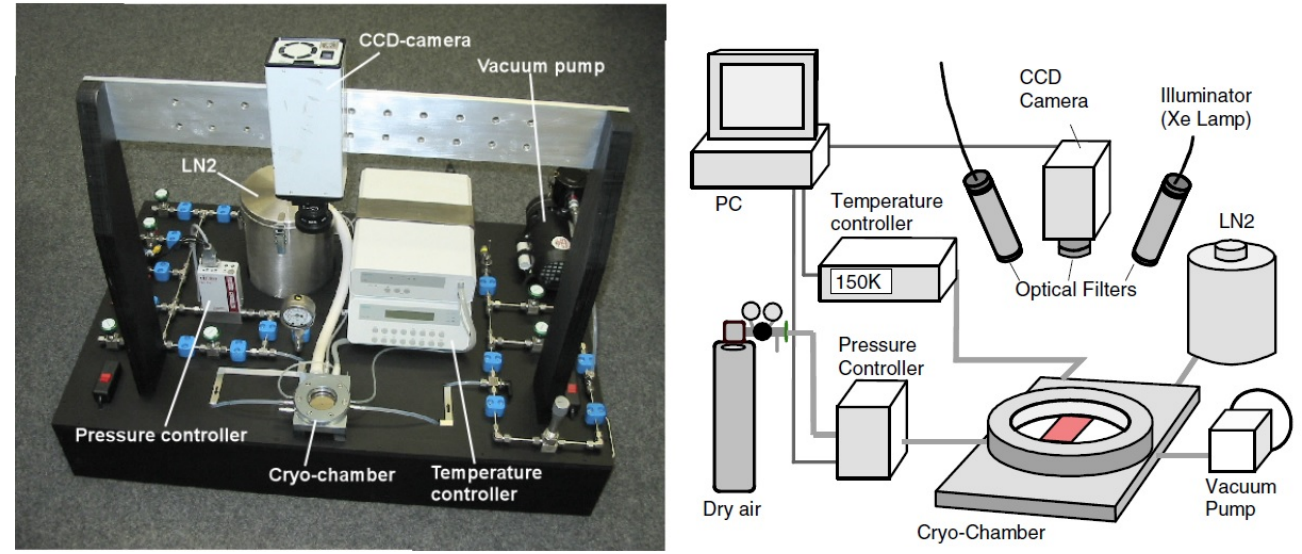

Figure B.4: Photo (left) and schematic setup (right) of the calibration chamber. (By courtesy of IOP Publishing from Egami et al. (2012))

intensity can be increased by a factor of ten when LEDs and filters are replaced.

In order to check the temperature sensitivity of the TSP with the new optical components, the negative relative intensity gradient was compared. It was found that the temperature sensitivity at high temperatures is not significantly altered by the used optical components (Risius et al., 2015a).

\section{B.2.4 Cameras}

The new CMOS camera PCO.edge was tested as a candidate to achieve a higher signal-to-noise ratio and temporal and spatial resolutions of TSP measurements in DNW-KRG. It was the first time that a CMOS camera was used for a quantitative TSP measurement with the Ruthenium based TSP, described in Sect. B.1.4. As the operation principle of CMOS cameras is significantly different from a CCD camera (Asai et al. 2017) the PCO.edge was tested thoroughly and compared with two CCD cameras that were known to be capable of quantitative pressure-sensitive paint measurements: PCO.pixelfly and PCO.sensicam.

In order to allow a comparison of the cameras a TSP sample was calibrated simultaneously with all cameras in the calibration chamber, described in Sect. B.2.1. It can be seen from Fig. B.10 that the normalized intensity of all cameras is similar. As a result the relative temperature gradient measured by the different camera models is almost identical, making it possible to use the same temperature calibration curve for all cameras tested (Risius, 2014).

\section{B.2.5 Periscope setup in DNW-KRG}

The original TSP measurement setup for DNW-KRG comprised the PCO.pixelfly cameras which were mounted directly into the flange, which used to fix the wind tunnel model and located in the side wall of the test section (see Fig. B.11 and Costantini et al. (2012); Fey et al. (2007)). The advantage of this measurement setup is that it can be easily installed. However, the setup is limited to the use of the PCO.pixelfly cameras which have a limited temporal and spatial resolution (and which are not manufactured anymore). Therefore, a new measurement setup was designed, which 


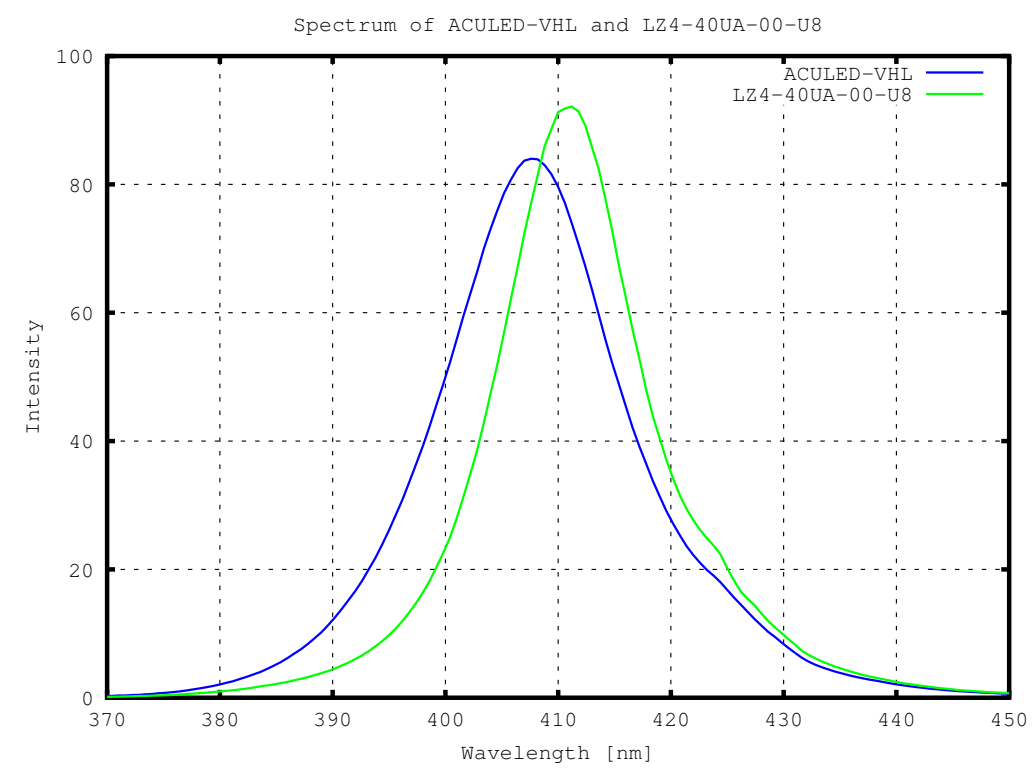

Figure B.5: Normalized emission spectra of old LEDs ACULED-VHL (blue) and new LEDs LED ENGIN LZ4-40UA00-U8 (green) in arbitrary not comparable units (Risius et al., 2015a)

allows the installation of larger camera models that have a higher temporal and spatial resolution (Risius, 2014).

The new persicope setup is shown in Figs. B.12-B.13. In the final version of the setup a Scheimpflug adapter (not shown here) was mounted between the lens and the PCO.edge camera in order to achieve a larger depth-of-focus. Therfore, the cameras were not mounted horizontally as shown in the CAD drawings (Figs. B.12-B.13), but in an angle to the horizontal plane.

\section{B.2.6 Result images of the advanced TSP measurement system}

As described above, the new measurement setup has the capability to increase, signal-to-noise ratio, spatial and temporal resolution of TSP result images. In order to visualize the improved image quality, which was achieved by installation of the new optical components (LEDs and optical filters), two result images are compared in Fig. B.14. It can be seen that the transition location can be distinguished much clearer with the improved result images.

The additional improvements in image quality achieved by the periscope setup are also visualized in Fig. B.14. The signal-to-noise ratio is further improved by the periscope setup because larger camera models can be used. Additionally, result images can be selected which are less influenced by sidewall effects and unstable flow conditions due to the improved temporal resolution. The spanwise variation of the transition location, caused by the three-dimensionality of the transition process, is clearly visible in the result obtained with the periscope setup.

A further improvement of the periscope setup is visualized in Fig. B.15. It can be seen that in the case of the old setup (left image) only a small part of the acquired image is used for TSP data evaluation (marked by a red box) while the rest of the image is not used.Note that the second half of the model is acquired by a second camera. In case of the persicope setup over $90 \%$ of the acquired 


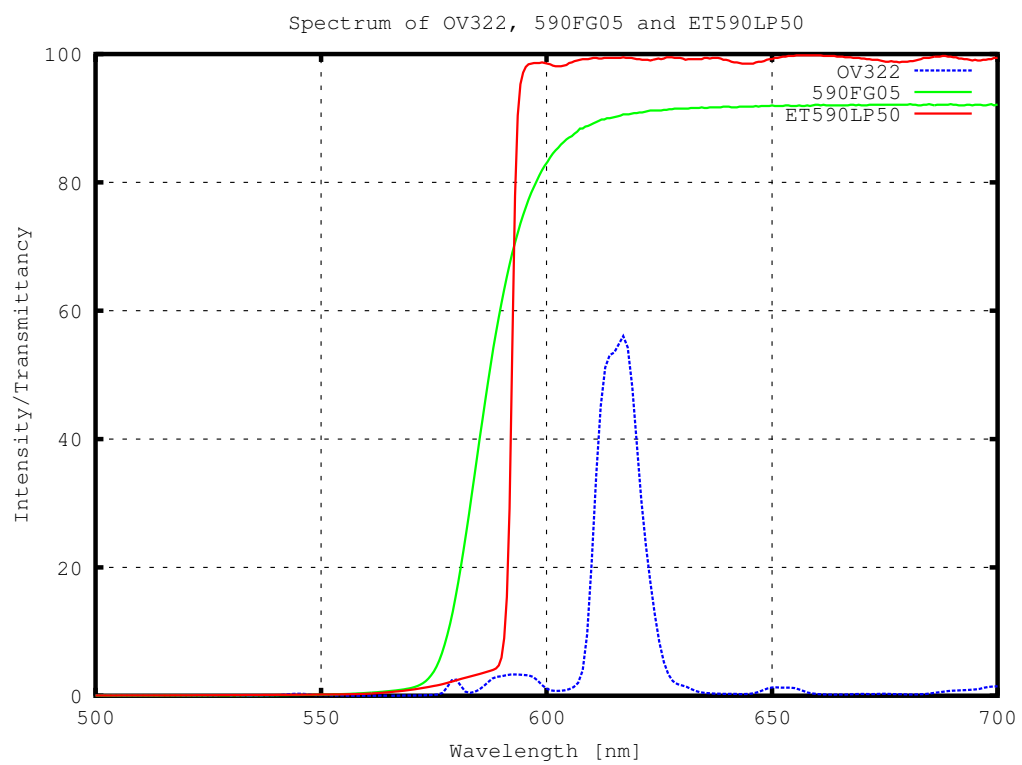

Figure B.6: Emission spectra of the Ruthenium based TSP (blue) and transmission spectra of the old camera filter 590FG05 (green) and new camera filter ET590LP (red) in arbitrary units (Risius et al., 2015a)

image is used for data evaluation on each side of the wind tunnel which results in a more efficient use of data storage.

\section{B.2.7 Spanwise transition detection}

The capability to determine the transition locations accurately was of fundamental importance for the results in this study, as described in Chapters 4 and 5 In order to achieve an exact measurement of transition locations an algorithm was developed which determines the maximum intensity gradient in chordwise direction (Costantini et al., 2018). In earlier studies of laminar-turbulent transition in DNW-KRG with TSP the transition detection was applied only to selected spanwise locations (Costantini, 2016, Costantini et al., 2012, 2016b). As part of the work for this study, the transition detection algorithm was extended to a transition detection over the complete span of the model. The two different approaches are visualized in Fig. B.16. On the left of Fig. B.16, five span wise locations were selected and the chordwise intensity distributions measured by TSP were averaged between each two lines of identical color. In the new approach, as shown on the right hand side, intensity distributions were determined at up to three hundred span wise locations. Regions with turbulent wedges or other disturbances were excluded from the analysis. Each transition location detected in chordwise direction was marked by a single red dot. This achievement made it possible to determine not only transition locations at higher accuracy but also to determine the standard deviation of measured transition locations. The improvement was an important step for the determination of laminar-turbulent transition locations which allowed the quantitative analysis of Mach and Reynolds number effects as described in Chapters 4 and 5 . The transition detection algorithm is described in more detail by Costantini et al. (2018). 


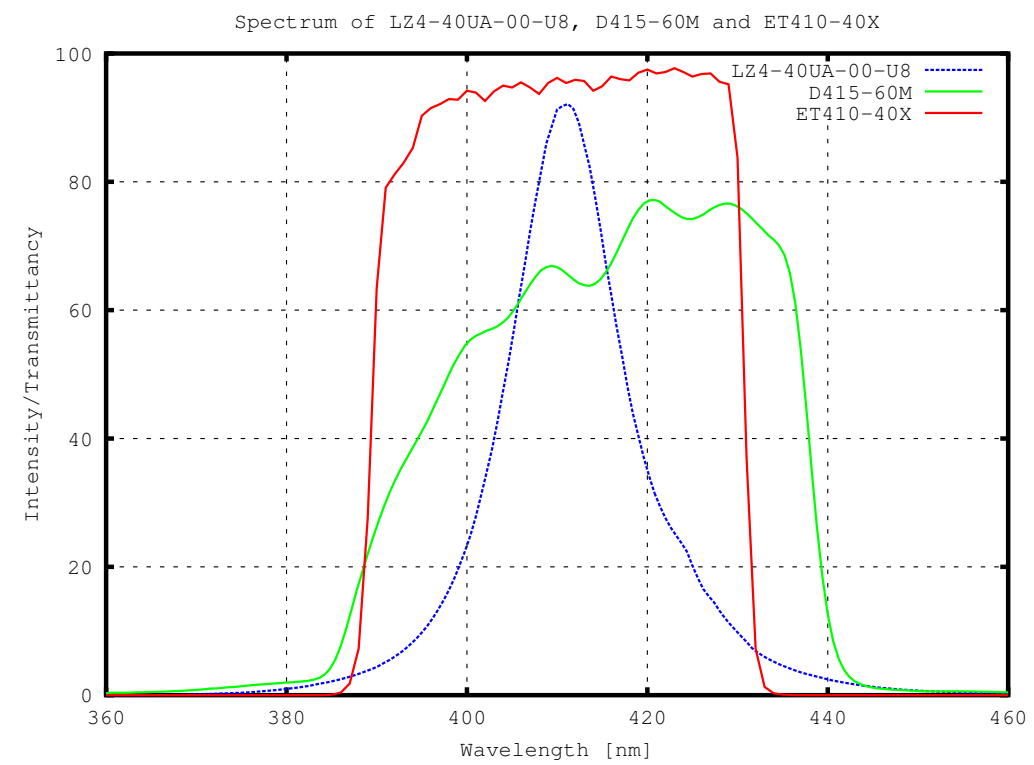

Figure B.7: Emission spectra of LED ACULED-VHL (blue), old LED filter D415/60M (green) and new LED filter ET410/40X (red) in arbitrary units (Risius et al., 2015a)

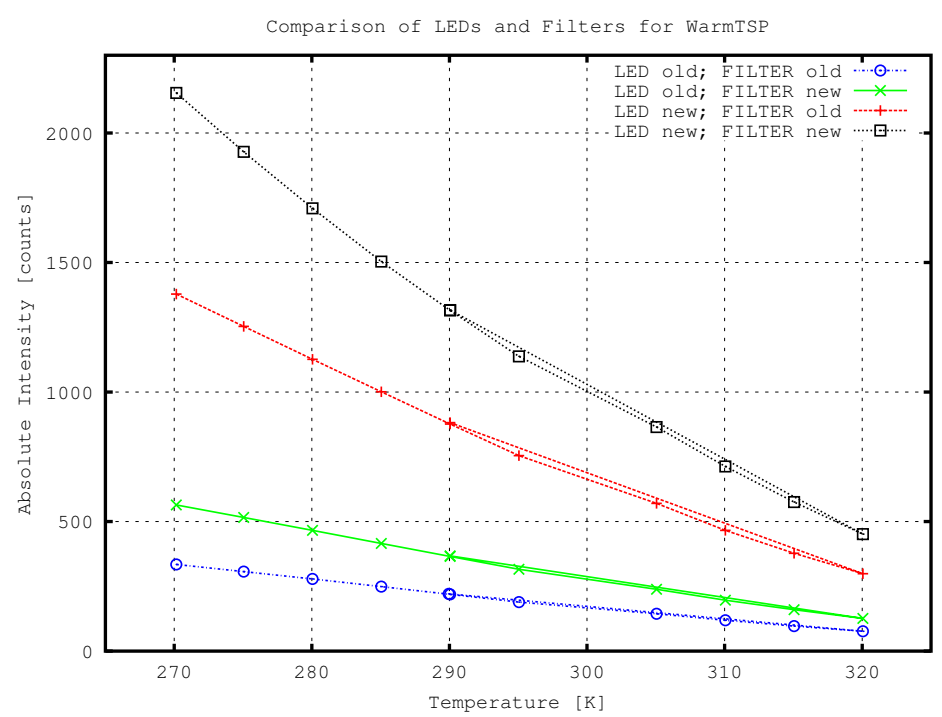

Figure B.8: Comparison of absolute intensity measured by old LEDs ACULED-VHL with old LED filters $D 415 / 60 M$ (blue), old LEDs ACULED-VHL with new LED filters ET410/40X (green), new LEDs LED ENGIN LZ4-40UA00-U8 with old LED filters D415/60M (red) and new LEDs LED ENGIN LZ4-40UA00-U8 with new LED filters ET410/40X (black). In order to exclude a hysteresis effect the measurement point at $290 \mathrm{~K}$ was repeated (Risius et al., 2015a) 


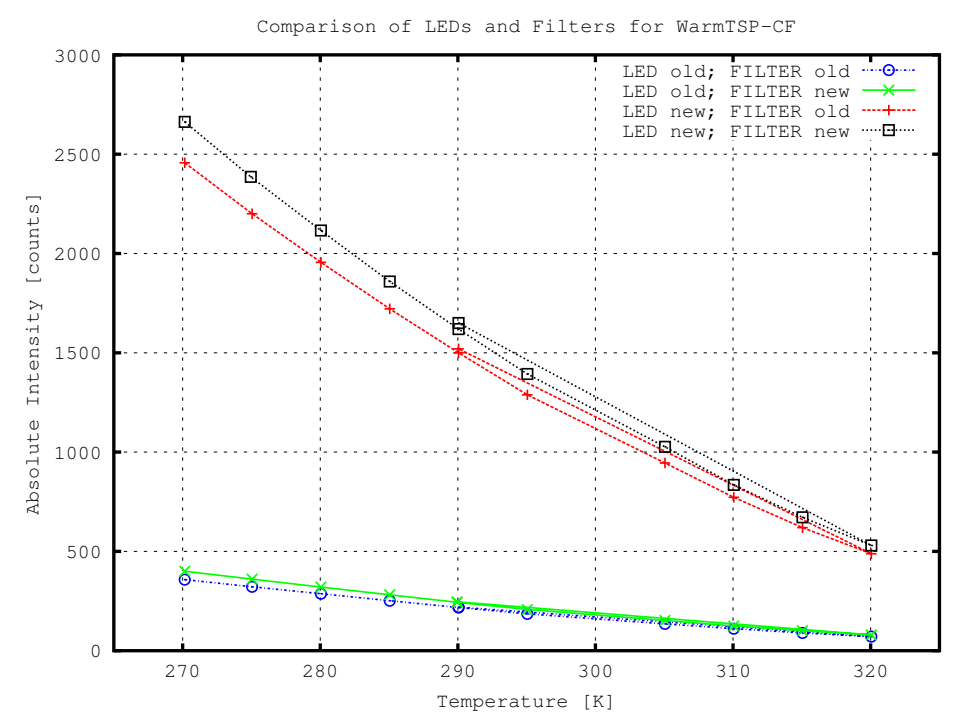

Figure B.9: Comparison of absolute intensity measured by old LEDs ACULED-VHL with old camera filters 590FG05 (blue), old LEDs ACULED-VHL with new camera filters ET590LP (green), new LEDs LED ENGIN LZ4-40UA00-U8 with old camera filters $590 F G 05$ (red) and new LEDs LED ENGIN LZ4-40UA00-U8 with new camera filters ET590LP (black). In order to exclude a hysteresis effect the measurement point at $290 \mathrm{~K}$ was repeated (Risius et al., 2015a)

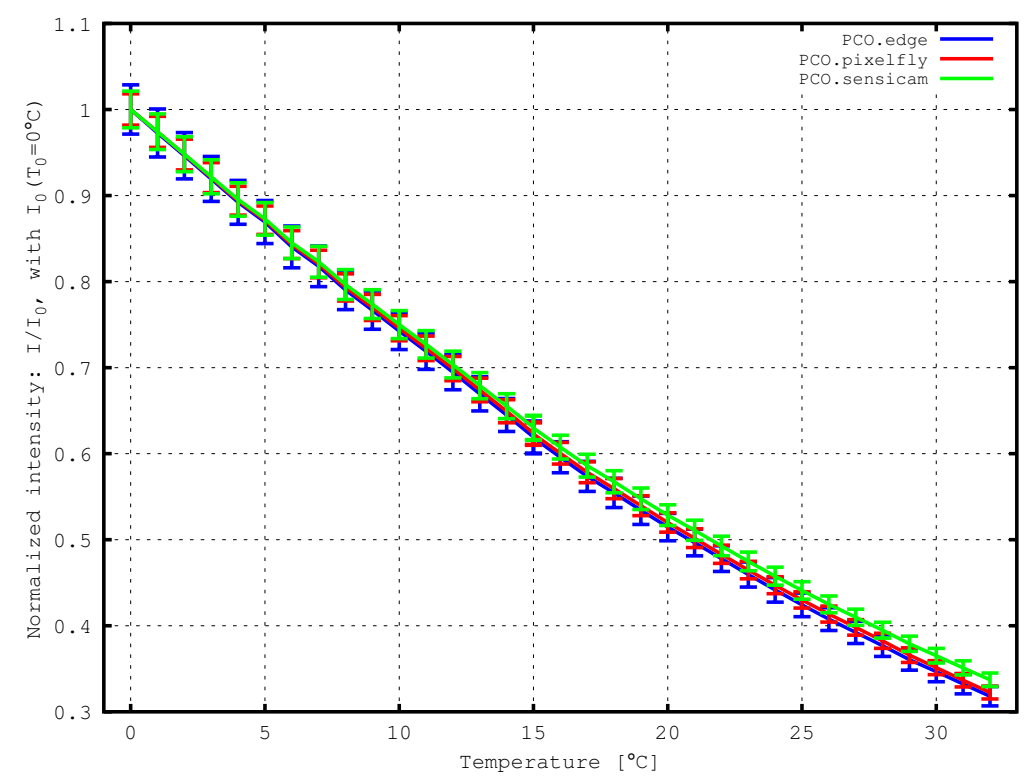

Figure B.10: Comparison of the normalized intensity measured with PCO.edge (blue), PCO.pixelfly (red) and PCO.sensicam (green) (Risius, 2014) 


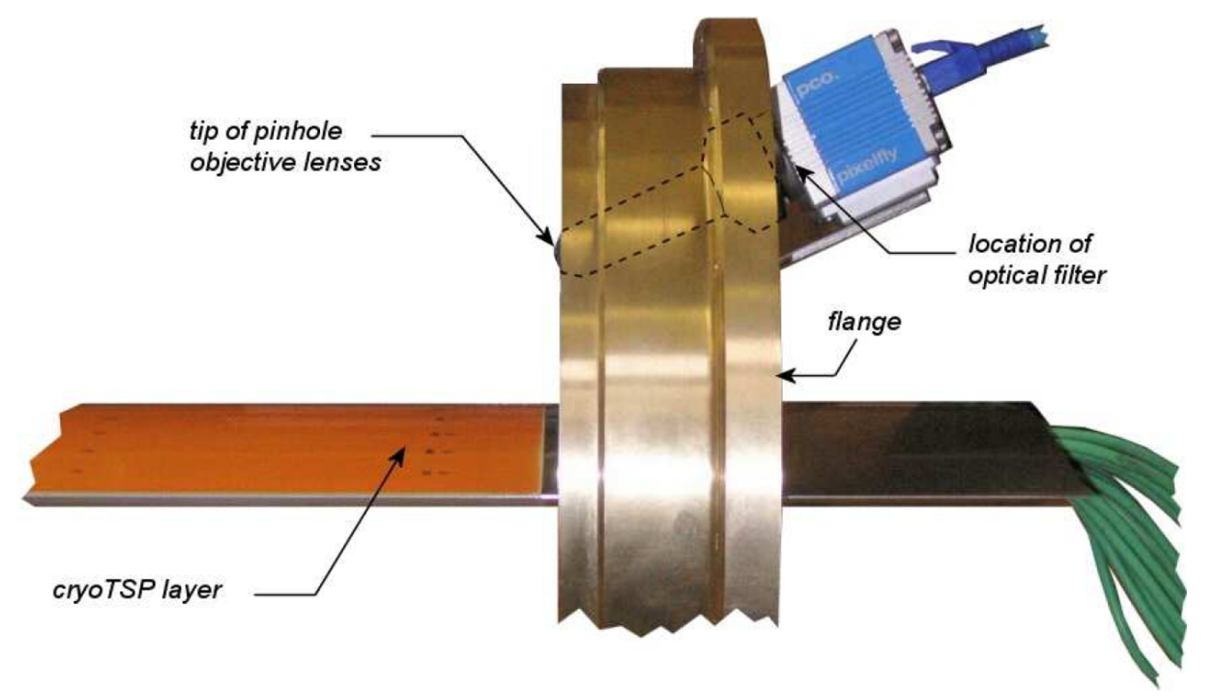

Figure B.11: Old setup with PCO.pixelfly mounted in the flange of the test section for TSP measurements in DNW-KRG (Costantini et al., 2012; Fey et al., 2007)
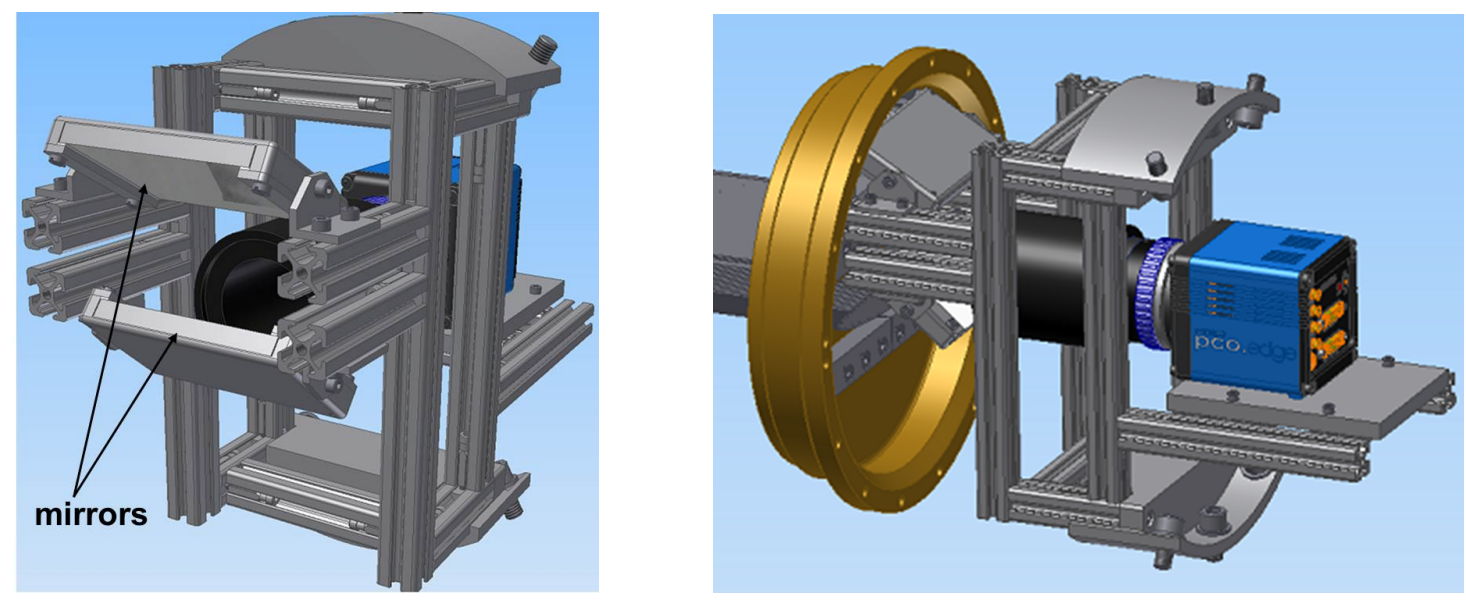

Figure B.12: CAD drawings of the new persicope setup in DNW-KRG. Two mirrors are located in front of the camera lens in order to view the upper side of the wind tunnel model 


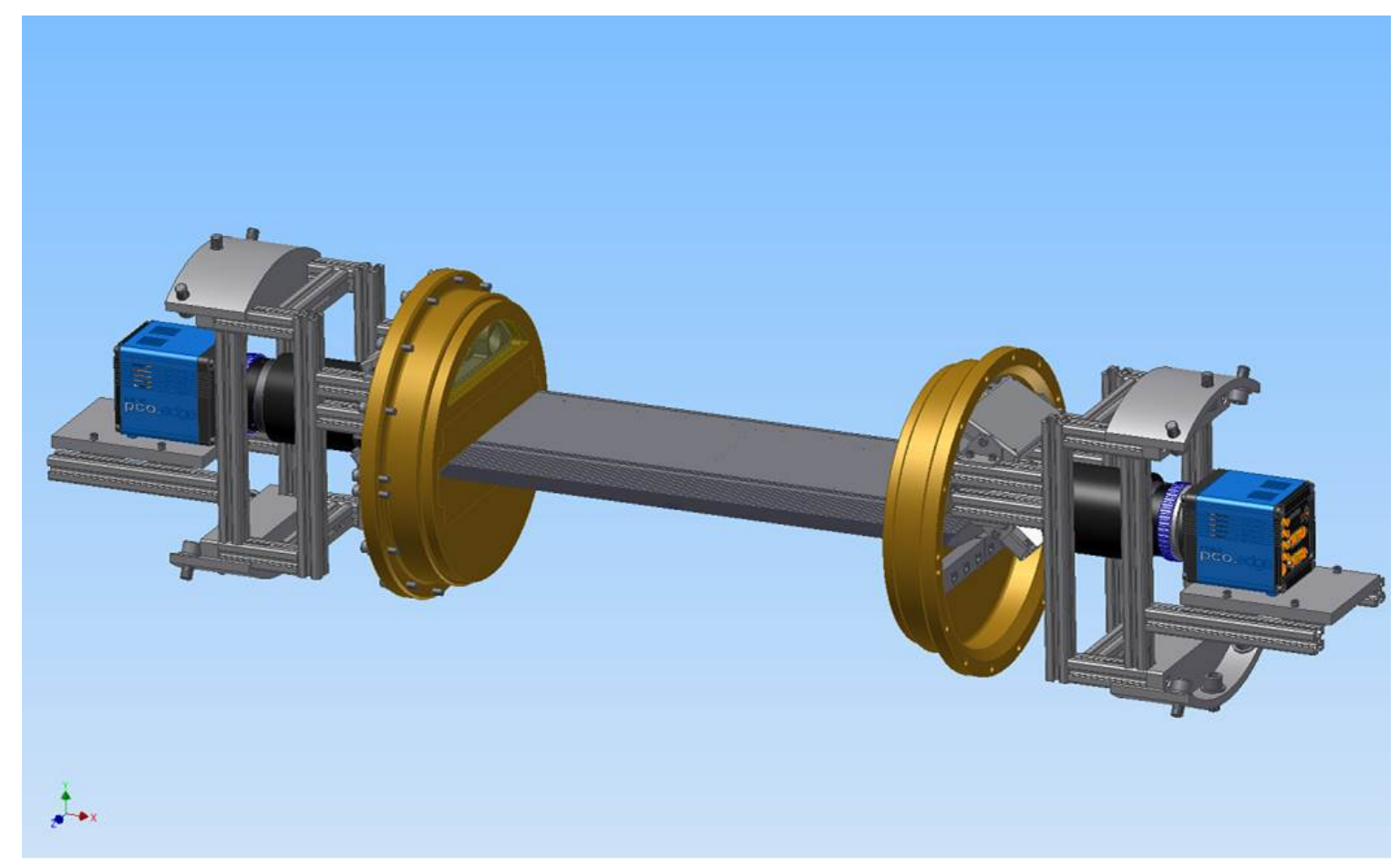

Figure B.13: Periscope setup for TSP measurement in DNW-KRG with a wind tunnel model located in the middle
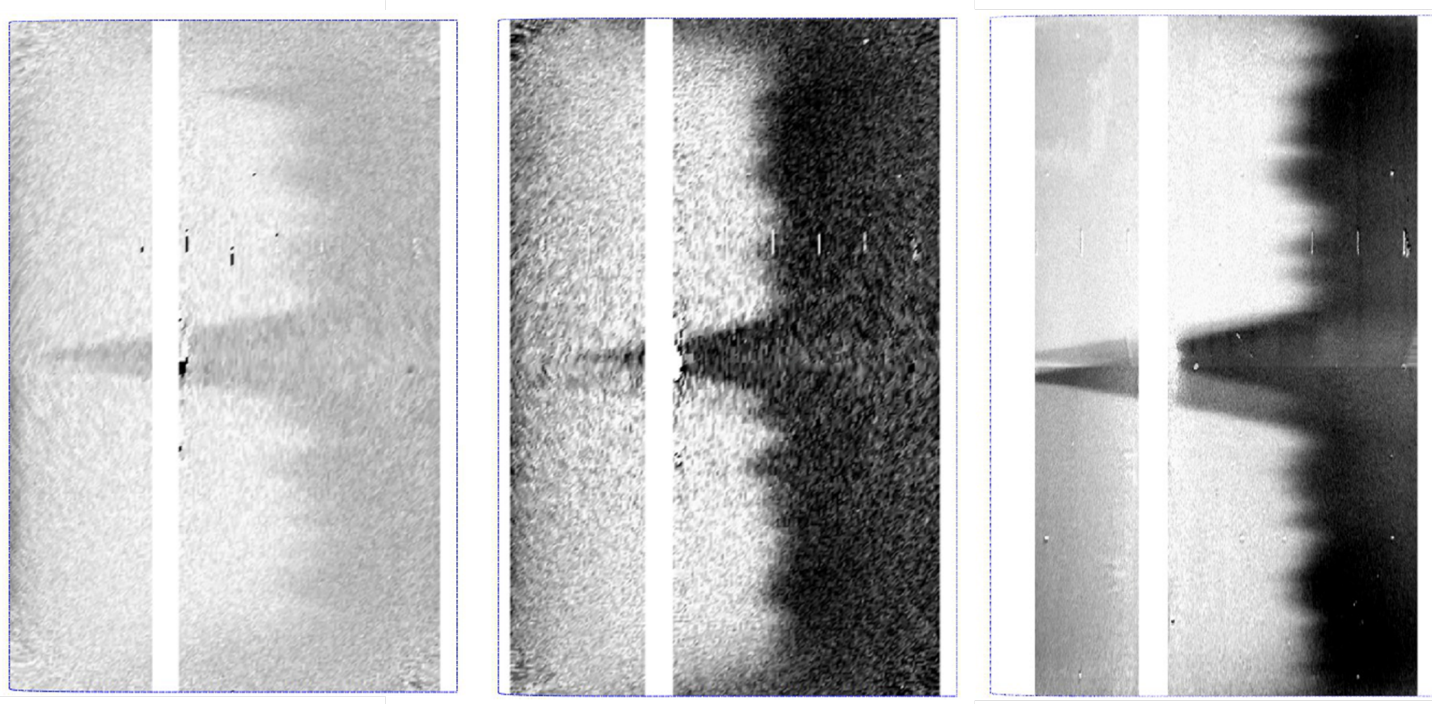

Figure B.14: View of PaLASTra model with the old LEDs and old setup (left), new LEDs and old setup (middle) and new LEDs and new persicope setup (right) in DNW-KRG. The images were acquired with the PaLASTra model in DNW-KRG at $M=0.35$ and $R e_{1}=17.5$ 


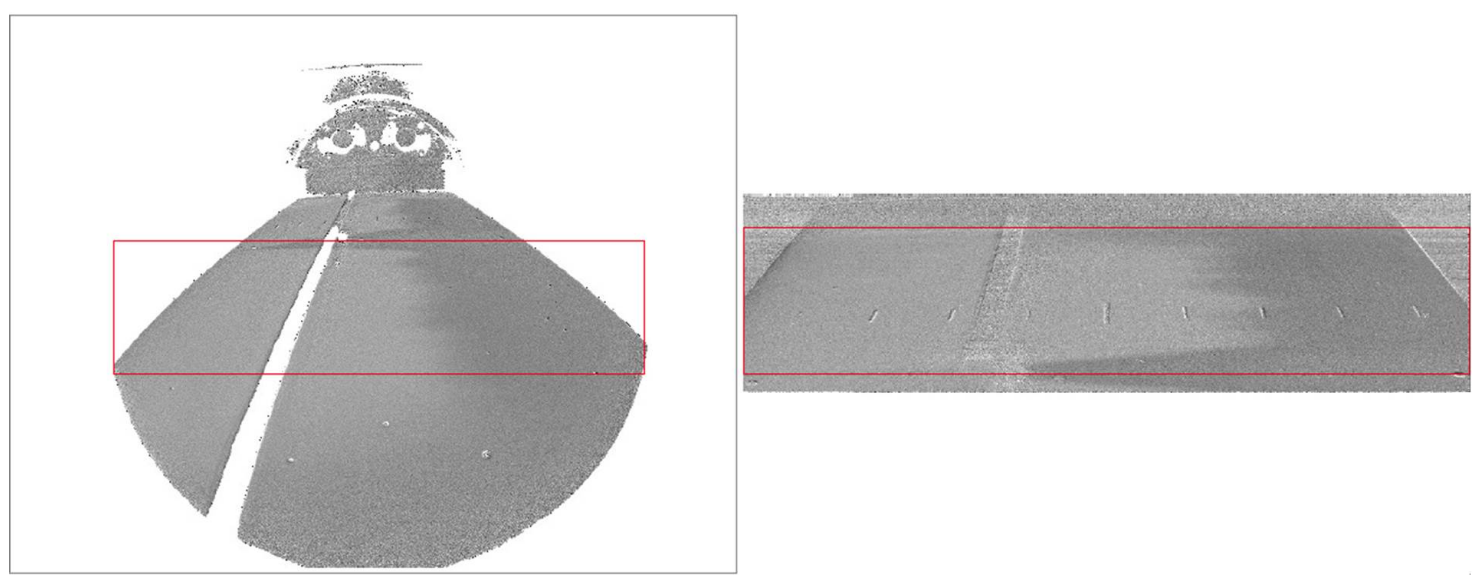

Figure B.15: View of PaLASTra model with the old setup (left) and new persicope setup (right) in DNW-KRG. The region used for TSP data evaluation is marked by a red box
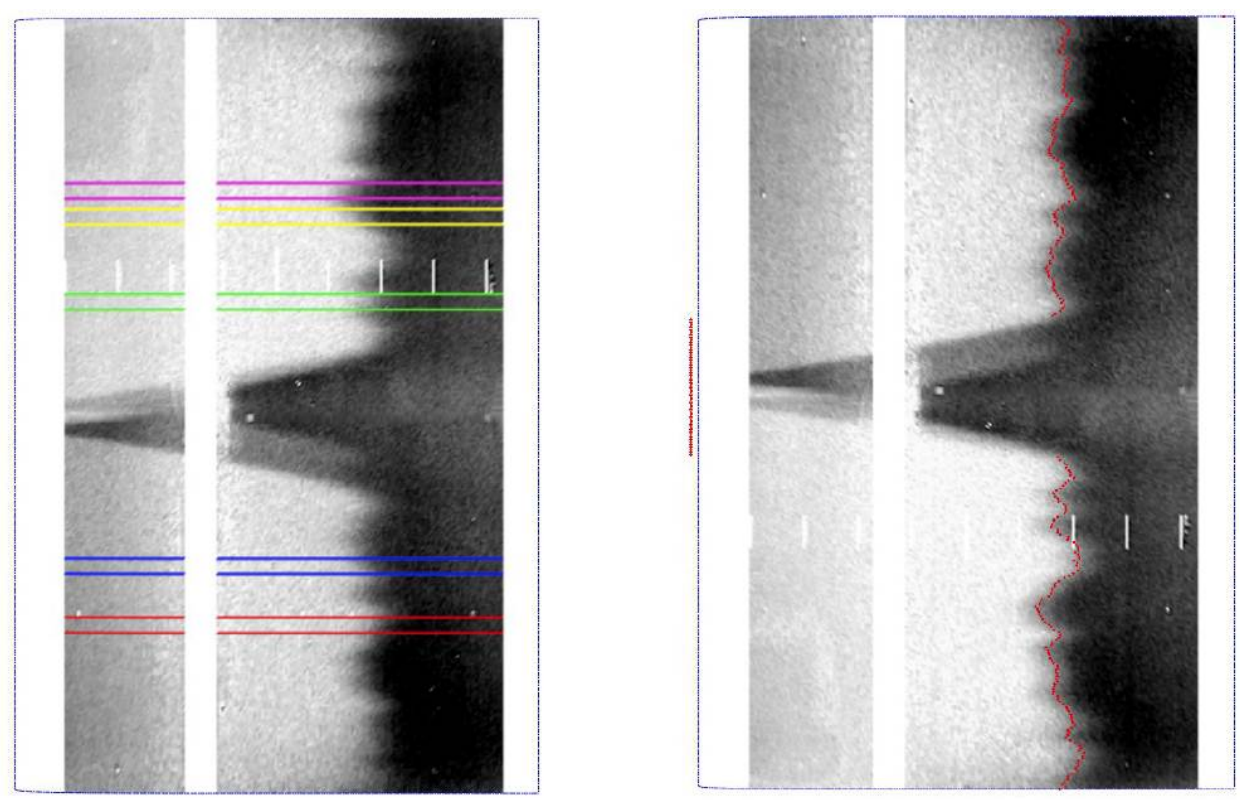

Figure B.16: Comparison of transition detection at five selected spanwise locations (left) and transition detection over the complete span at up to three hundred locations (right). The images were acquired with the PaLASTra model in DNW-KRG at $M=0.35$ and $R e_{1}=17.5$ 